\title{
Comparative Cladistics:
}

\section{Fossils, Morphological Data Partitions}

\section{and Lost Branches in the Fossil Tree of Life}

\author{
Ross Callum Parker Mounce \\ http://orcid.org/0000-0002-3520-2046 \\ A thesis submitted for the degree of Doctor of Philosophy \\ University of Bath \\ Department of Biology and Biochemistry \\ October 2013
}

Copyright:

(C) 2014 Ross Mounce. This is an open-access thesis distributed under the terms of the Creative Commons Attribution License, which permits unrestricted use, distribution, and reproduction in any medium, provided the original author and source are credited.

Signature:

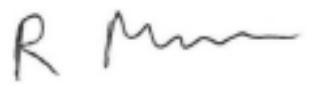




\section{Table of Contents}

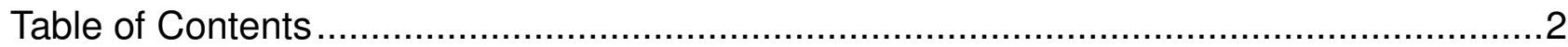

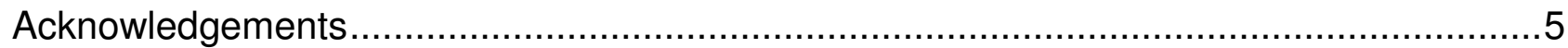

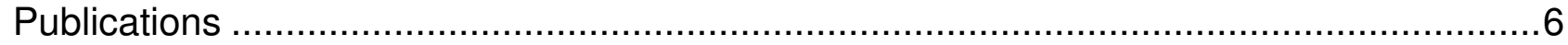

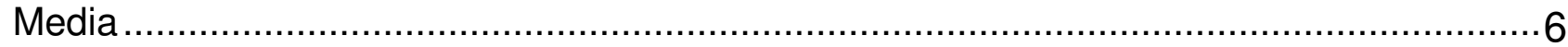

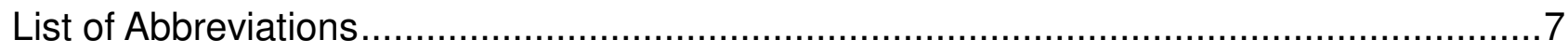

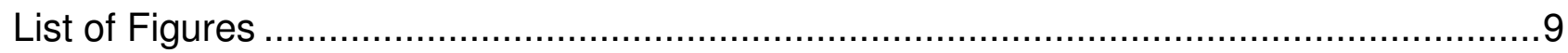

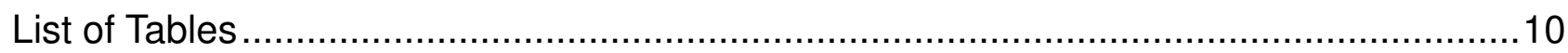

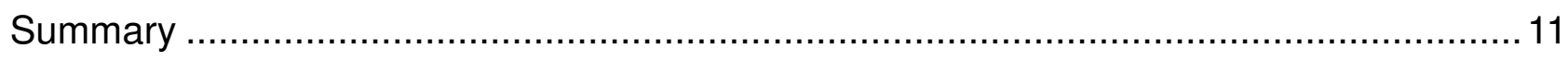

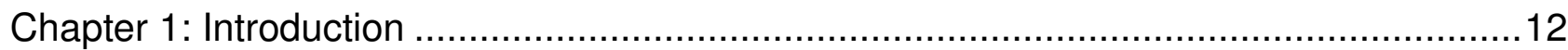

1.1 Overview of Inferring Evolutionary Relationships in Palaeontology ...................13

1.2 Why not just use molecular data? ............................................................ 16

1.3 Is Morphology Actually Useful for Reconstructing Phylogeny? ........................16

1.4 Aims of this thesis ............................................................................... 19

The Congruence of Cranial \& Postcranial Characters in Vertebrate Phylogeny ...............21

2.1 Abstract................................................................................................ 21

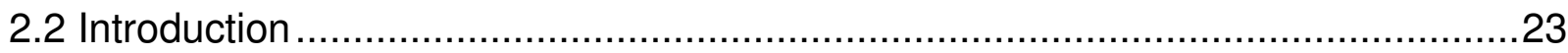

2.2.1 Congruence between Partitions of Morphological Data .................................24

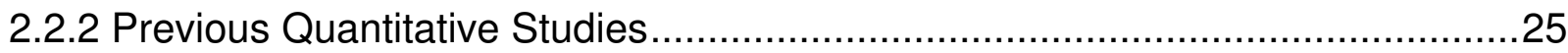

2.2.3 Why Examine the Congruence of Cranial and Postcranial Partitions? ..............27

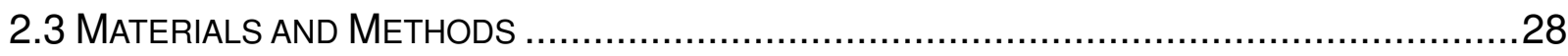

2.3.1 Do Cranial and Postcranial Characters Imply Different Levels of Homoplasy?..29 2.3.2 Is there More Conflict Between Cranial and Postcranial Characters than we Might Expect? .......................................................................................... 34

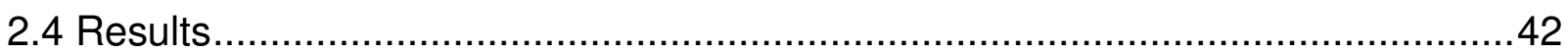

2.4.1 Homoplasy and Resolution in Cranial and Postcranial Data Partitions.............43

2.4.2 Congruence between Cranial and Postcranial Signals (ILD tests)....................44

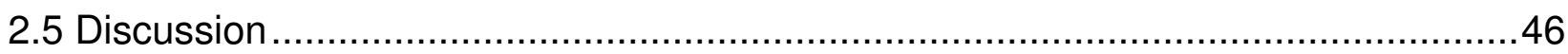

2.5.1 A Significant Minority of Cranial and Postcranial Partitions have Incongruent

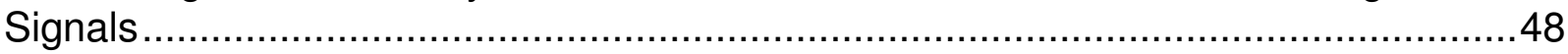

2.5.2 Cranial and Postcranial Partitions Imply Significantly Different Relationships in a

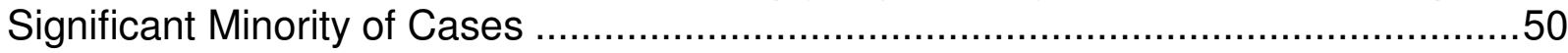

2.5.3 What do these Results Imply for Cladistic Analyses of Morphology?...............51

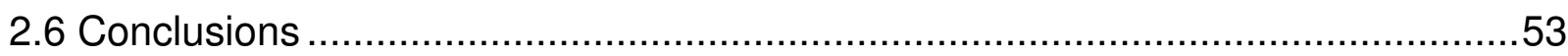


Chapter 3: An Updated Examination of the Impact of the Fossil Taxa in Parsimony Analyses of Morphology

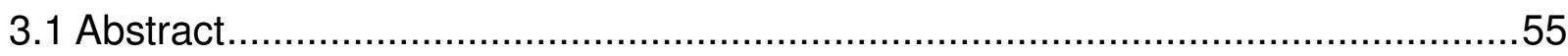

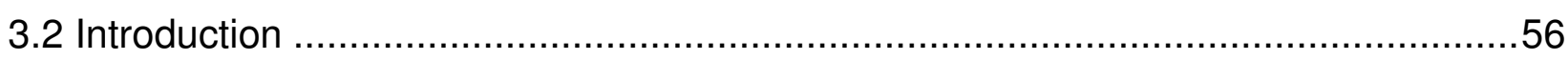

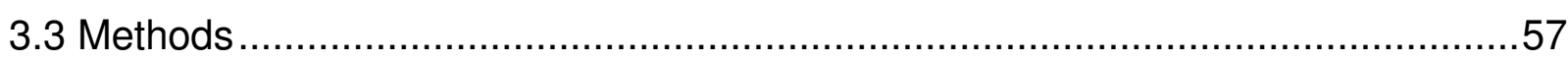

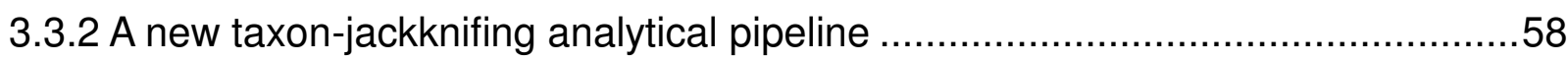

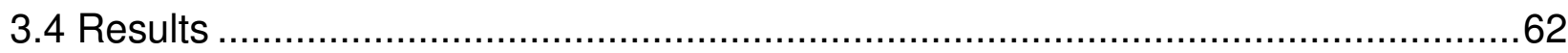

3.4.1 On average Fossil and Extant Taxa have remarkably similar Topological Impact as judged by single-taxon deletions.

3.4.2 Fossil Taxa on average do not increase the number of MPTs any more than Extant Taxa . .65

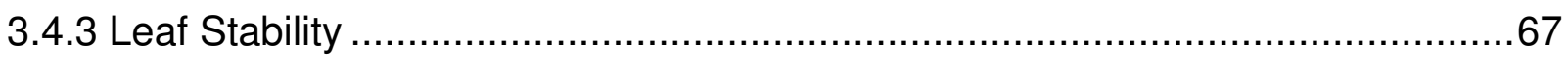

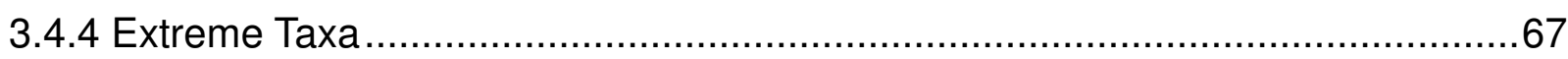

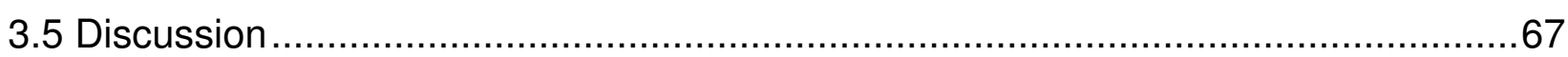

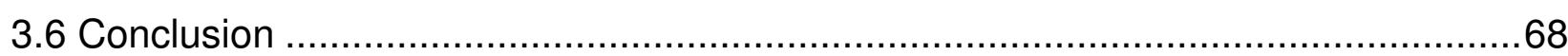

Chapter 4: A review of the ILD randomisation test: uses and abuses ..........................69

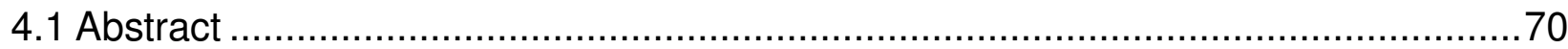

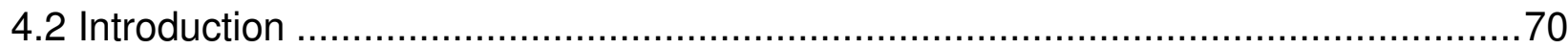

4.2.1 Historical Importance and Context ..................................................... 71

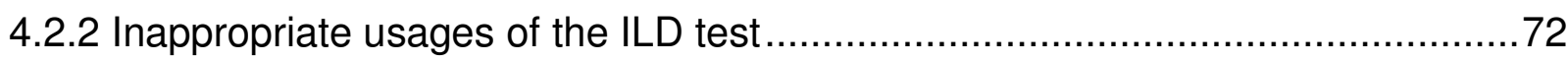

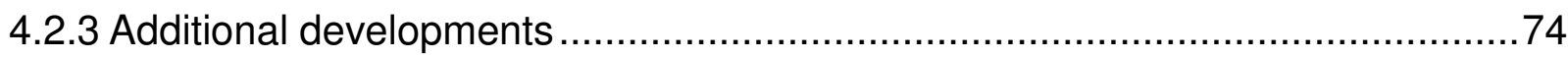

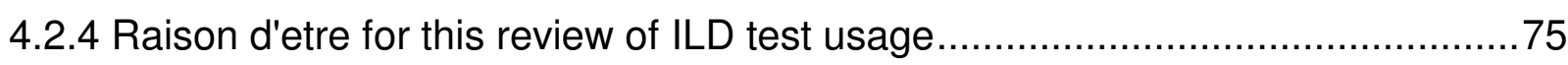

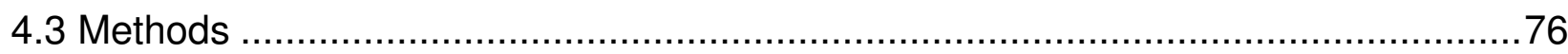

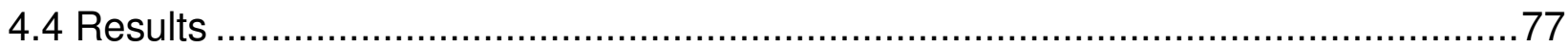

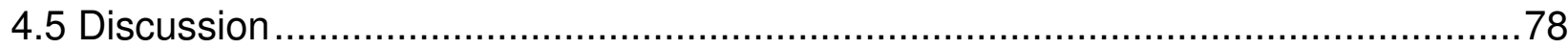

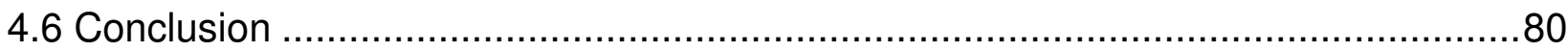

Chapter 5: A modification of Archie's Homoplasy Excess Ratio in the presence of missing data

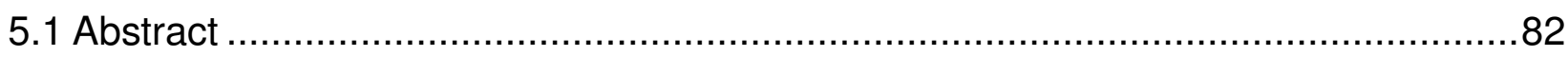

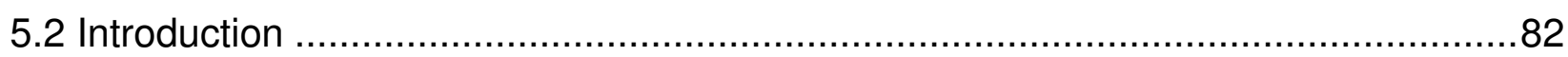

5.2.1 The Problem of Permuting Missing / Inapplicable Data in Matrices ..................84

5.2.2 Time-Efficient Computational Implementation............................................. 87

5.2.3 How many replications are needed for a robust MEANNS estimate? ...............87

5.2.4 Is the HER missing data problem significant in real matrices? ........................88

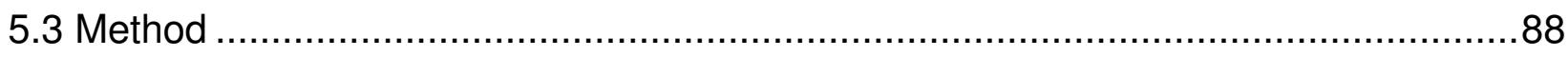

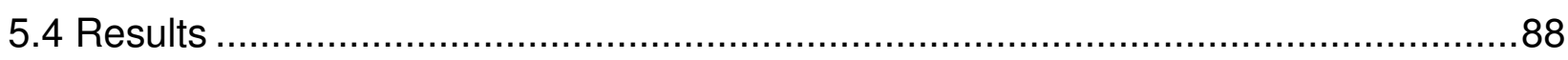

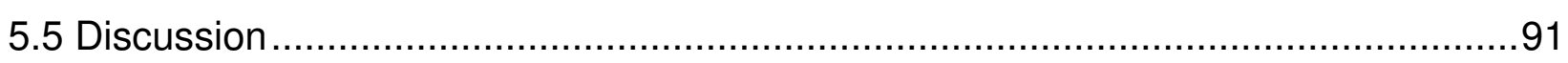

5.5.1 Why has HER seemingly been ignored for so long? .............................. 91 
Chapter 6: Optimal search strategies for finding phylogenetic data .93

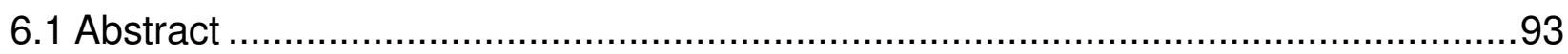

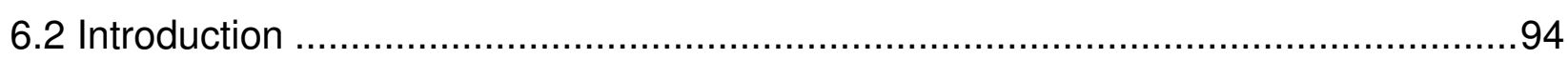

6.2.1 Preliminary Investigation.................................................................... 95

6.2.2 Scirus does not cover many important natural history journals .......................97

6.2.3 The long-tail of phylogenetic content ................................................... 98

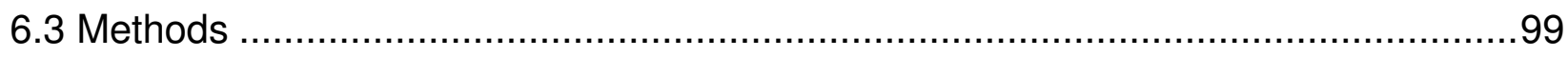

6.3.1 Testing the computational efficiency of local full-text search options ...............100

6.3.2 Justification of search terms used, and the use-case .................................101

6.3.3 Distinguishing between morphology-based \& molecular-only analyses...........102

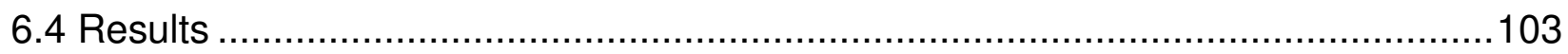

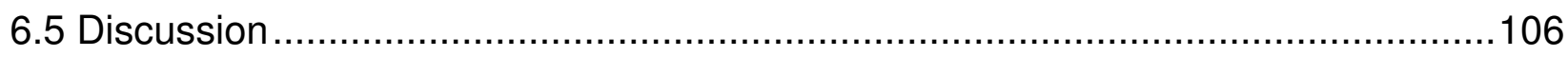

6.5.1 What exactly is WoK missing? Does it matter?.......................................106

6.5.2 What about the non-journal data archives? Do they have the data? ..............107

6.5.3 Few morphology-using phylogenetic analyses in BMC journals .....................108

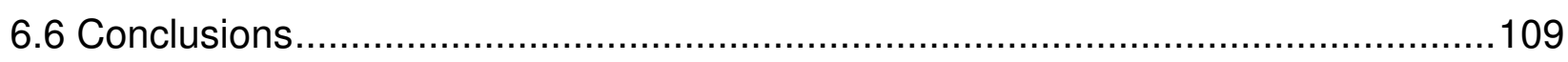

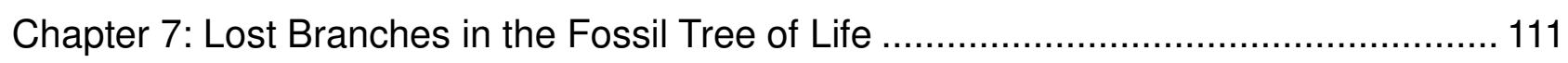

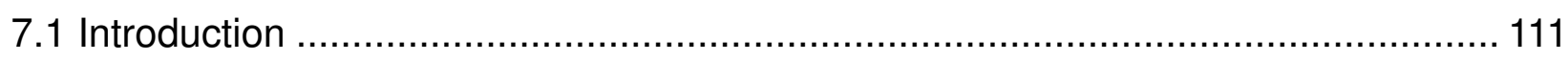

7.2 How many morphology-based phylogenetic studies are there? .......................... 111

7.3 How much data from morphology-based phylogenetic studies is publicly available?

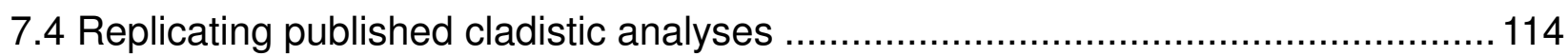

7.5 Comments on the Liu et al. reply .......................................................... 114

7.5 Suggestions for the future of data publication and review ............................... 115

7.6 Positive reasons to share published data.................................................. 116

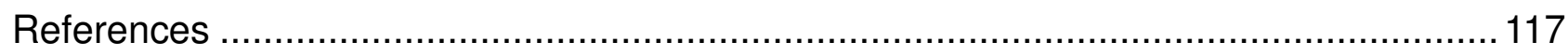

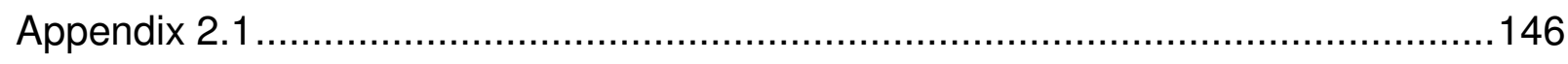

Appendix 3.1 Data to reproduce PAUP bug ................................................ 147

Appendix 3.2 Extra Summary Data......................................................... 148

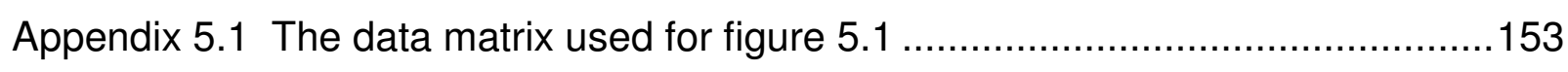

Appendix 5.2 Script to implement selective-permutations ' mher.run ' ......................153

Appendix 5.3 Script to calculate MHER in one command 'get-mher.sh' .................155

Appendix 6.1 Demonstrating grep .............................................................. 157

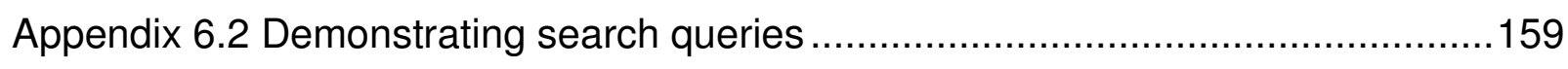

Appendix 6.3 Google Scholar finds IR copies................................................ 159

Appendix 7.1 Phylogenetic position of Diania challenged..................................160 


\section{Acknowledgements}

I would like to thank my supervisor, Matthew Wills for putting up with me all this time. I have been lucky to have such accommodating and understanding support. I also must thank my lab mates Martin Hughes, Anne O'Connor, Sylvain Gerber, Katie Davis, Rob Sansom and everyone else in the Biodiversity Lab at the University of Bath - we had some great times and some brilliant times together. Sincere thanks also to the University of Oslo Bioportal computing cluster for providing me free cloud computation for my work.

Many people have helped spur my imagination along the way with ideas for different chapters of this thesis. For this I would like to thank Ward Wheeler, Pablo Goloboff, Mark Siddall, Dan Janies, Steve Farris and the generous financial support of the Willi Hennig Society. I want to thank all those in the palaeontology community who have shared their published data with me, particularly Graeme Lloyd for his stirling work in making dinosaurian data available - I hope I have done something interesting with the data I have used and opened eyes to new possibilities. I also want to thank all those in the open science community - Peter Murray-Rust, Todd Vision, Heather Piwowar, Mark Hahnel, Martin Fenner, Geoffrey Boulton, Jenny Molloy and so many more I've had the pleasure of meeting in person. The energy and enthusiasm I drew from countless online discussions on Facebook, Google+ and Twitter was truly inspirational.

For facilitating greater access to scientific literature I must heartily thank the Natural History Museum, London library and archives, the \#icanhazpdf community on Twitter, Wikipaleo on Facebook, References Wanted on FriendFeed, Library.nu, and SciHub. Without these additional literature access facilitators I would not have been able to read half the sources I cite in this thesis.

I must thank my wife Avril for her patience with me especially during the write-up phase, for allowing me to go away to all these amazing conferences abroad, and for tolerating all those long nights into mid-morning when I was tapping away on my noisy keyboard. Finally, I thank my family: Richard, Rosemary \& Tara for repeatedly encouraging me to finish my thesis - I got there in the end! 


\section{Publications}

- Mounce, R. C. P. and Wills, M.A. 2011 Phylogenetic position of Diania challenged. Nature 476:E1.

- Mounce, R., Butler, A., Davis, K., Dunhill, A., Garwood, R., Lamsdell, J., Legg, D., Lloyd, G., Pittman, M., Warnock, R., and Wolfe, J. 2011. An open letter in support of palaeontological digital data archiving. http://supportpalaeodataarchiving.co.uk/ ( featured in Nature News: 'Fossil data enter the web period' doi:10.1038/472150a )

- Stoltzfus, A., O'Meara, B., Whitacre, J., Mounce, R., Gillespie, E., Kumar, S., Rosauer, D., and Vos, R. 2012. Sharing and re-use of phylogenetic trees (and associated data) to facilitate synthesis. BMC Research Notes Vol. 5, 1-15.

- Mounce R. 2012. Twitter for Systematists. The Systematist Vol. 34, 14-15.

- Mounce, R. 2012. Life as a palaeontologist: Academia, the internet and creative commons. Palaeontology Online Vol. 2, 1-10.

- Mounce, R. 2013. Open access and altmetrics: distinct but complementary. Bulletin of the American Society for Information Science and Technology Vol. 39, 14-17.

- Mounce, R. and Janies, D. 2013. Synergistic sharing of data and tools to enable team science. In: J. Zander and P. J. Mosterman (eds.) Computation for Humanity: Information Technology to Advance Society. CRC Press, 437-447.

- Poisot, T., Mounce, R. and Gravel D. 2013. Moving toward a sustainable ecological science: don't let data go to waste! Ideas in Ecology \& Evolution Vol. 6, 11-19.

- Mounce, R. and Wills, M. A. (in revision) Congruence of Cranial and Postcranial Characters in Vertebrate Phylogeny

\section{Media}

Radio: BBC Radio 3 Night Waves http://bit.ly/NightWaves [live, 2012-10-02] Voice of Russia radio http://bit.ly/VoRussia [recorded as live, 2012-08-17]

Press mentions in: Publishing: Open to possibilities [Nature Jobs, 2013-03-27]

Researchers opt to limit uses of open-access publications ～[Nature News, 2013-02-06] Text-mining spat heats up

[Nature News, 2013-03-20] 


\section{List of Abbreviations}

\begin{tabular}{|c|c|}
\hline AAAS & American Association for the Advancement of Science \\
\hline $\mathrm{AgD}$ & Agreement Subtree Distance \\
\hline AMNH & American Museum of Natural History \\
\hline ASIH & American Society of Ichthyologists and Herpetologists \\
\hline ASPT & American Society of Plant Taxonomists \\
\hline *BEAST & Bayesian Evolutionary Analysis by Sampling Trees (software) \\
\hline BMC & BioMed Central \\
\hline $\mathrm{CFI}$ & Consensus Fork Index \\
\hline $\mathrm{Cl}$ & Consistency Index (ensemble) \\
\hline CSIRO & Commonwealth Scientific and Industrial Research Organisation \\
\hline CUP & Cambridge University Press \\
\hline DNA & Deoxyribonucleic acid \\
\hline $\mathrm{GHz}$ & Gigahertz \\
\hline GIC & Group inclusion characters \\
\hline GNU & GNU's Not Unix! \\
\hline GS & Google Scholar \\
\hline GUI & Graphical User Interface \\
\hline HDD & Hard Disk Drive \\
\hline HER & Homoplasy Excess Ratio \\
\hline HERM & Homoplasy Excess Ratio Maximum \\
\hline HTML & HyperText Markup Language \\
\hline $\mathrm{I} / \mathrm{O}$ & Input/Output \\
\hline ILD & Incongruence Length Difference \\
\hline IRD & Incongruence Relationship Difference \\
\hline $\mathrm{KH}$ & Kishino-Hasegawa \\
\hline MAS & Microsoft Academic Search \\
\hline MAW & Dr Matthew A. Wills (supervisor) \\
\hline MEANNS & Mean Optimal Tree Length Inferred after Matrix Randomizations \\
\hline MEGA & Molecular Evolutionary Genetics Analysis (software) \\
\hline MF & Mickevich-Farris \\
\hline MHER & Modified Homoplasy Excess Ratio \\
\hline MINL & Minimum Possible Length \\
\hline
\end{tabular}




$\begin{array}{ll}\text { MPT } & \text { Most Parsimonious Tree } \\ \text { MS } & \text { Mendeley Search } \\ \text { MWU } & \text { Mann-Whitney U } \\ \text { NNID } & \text { Nearest Neighbour Interchange Distance } \\ \text { NONA } & \text { No Name (software) } \\ \text { NPG } & \text { Nature Publishing Group } \\ \text { OA } & \text { Open Access } \\ \text { OSI } & \text { Open Source Initiative } \\ \text { OUP } & \text { Oxford University Press } \\ \text { PAUP* } & \text { Phylogenetic Analysis Using Parsimony *and other methods (software) } \\ \text { PDF } & \text { Portable Document Format } \\ \text { PHYLIP } & \text { PHYLogeny Inference Package (software) } \\ \text { PICA } & \text { Phylogenetic Inference by Compatibility Analysis (software) } \\ \text { PLD / PD } & \text { Path Length Difference / Path Difference } \\ \text { PLOS } & \text { Public Library of Science } \\ \text { PMC } & \text { PubMed Central } \\ \text { PTP } & \text { Permutation Tail Probability } \\ \text { QD } & \text { Quartets Distance } \\ \text { RAS } & \text { Random Addition Sequences } \\ \text { RAxML } & \text { Randomized A(x)ccelerated Maximum Likelihood (software) } \\ \text { REHER } & \text { Relative Expected Homoplasy Excess Ratio } \\ \text { RF } & \text { Robinson-Foulds } \\ \text { RI } & \text { Retention Index } \\ \text { SSD } & \text { Solid State Drive } \\ \text { STEM } & \text { Species Tree Estmation using Maximum Likelihood (software) } \\ \text { TAXEQ3 } & \text { Safe TAXonomic reduction using taxonomic Equivalence (software) } \\ \text { TBR } & \text { Tree Bisection and Reconnection } \\ \text { TILD } & \text { Topological Incongruence Length Difference } \\ \text { TNT } & \text { Tree analysis using New Technology (software) } \\ \text { tsV } & \text { Unweighted Pair Group Method with Arithmetic Mean } \\ \text { UPGMA } & \text { Web of Knowledge } \\ \text { WoK } & \text { XML }\end{array}$




\section{List of Figures}

\section{Chapter 1:}

Fig. 1.1 Anomalocaris from the Mt Stephen Trilobite Beds

\section{Chapter 2:}

Fig. 2.1 Characters sampled from different anatomical regions can yield radically different most parsimonious trees (MPT) when analysed in isolation.

Fig. 2.2 The number of characters in a data matrix influences probable indices of homoplasy.

Fig. 2.3 Calculation of two inhomogeneity metrics for cranial and postcranial partitions of a hypothetical data set.

Fig. 2.4 Most parsimonious tree derived from the 103 cranial and 139 postcranial characters in the mammalian data of Beck (2008).

Fig. 2.5 Tree-to-tree distances for cranial and postcranial partitions of the mammalian data of Pujos (2007).

\section{Chapter 3:}

Fig. 3.1 Schematic diagram of the workflow that implements taxon deletion comparisons.

Fig. 3.2 Demonstrating a taxon with low leaf-stability.

Fig. 3.3 Measuring the relationships impact of removing single taxa

Fig. 3.4 A demonstration of a fossil taxon that helps 'support' a phylogeny

\section{Chapter 5:}

Fig. 5.1 The distribution of permuted matrix tree lengths for 1000 permutations

Fig. 5.2 A hypothetical sparse matrix of 19 parsimony-informative characters and 20 taxa.

Fig. 5.3 A possible permutation of the sparse matrix from figure 5.2

\section{Chapter 6:}

Fig. 6.1 Illustrating the long-tail distribution of phylogeny-related articles across hundreds of different journal titles indexed in WoK, from 2000-2011.

Fig. 6.2 Article PDF file size distribution of all articles in each of the corpora 


\section{List of Tables}

\section{Chapter 2:}

Table 2.1 Summary statistics for the 62 vertebrate morphological data sets analysed herein.

\section{Chapter 3:}

Table 3.1 Summary statistics for the 75 morphological data sets analysed herein from vertebrate, invertebrate and botanical studies.

\section{Chapter 5:}

Table 5.1 A comparison of measures of homoplasy, sorted by missing data, for 60 assorted data sets including 7 molecular data supermatrices

\section{Chapter 6:}

Table 6.1 A comparison of simple search results for the term "phylogeny" and "winclada" in scholarly documents published between the years $2000-2012$

Table 6.2 A demonstration of different local desktop full text search methods, as performed on the Zootaxa corpus

Table 6.3 Summary of local desktop full-text searches

Table 6.4 Comparison of recall between popular academic web literature search portals, to the known number of articles that contain the search term in the full text of the article 


\section{Summary}

In this thesis I attempt to gather together a wide range of cladistic analysis of fossil and extant taxa representing a diverse array of phylogenetic groups. I use this data to quantitatively compare the effect of fossil taxa relative to extant taxa in terms of support for relationships, number of most parsimonious trees (MPTs) and leaf stability. In line with previous studies I find that the effects of fossil taxa are seldom different to extant taxa although I highlight some interesting exceptions. I also use this data to compare the phylogenetic signal within vertebrate morphological data sets, by choosing to compare cranial data to postcranial data.

Comparisons between molecular data and morphological data have been previously well explored, as have signals between different molecular loci. But comparative signal within morphological data sets is much less commonly characterized and certainly not across a wide array of clades. With this analysis I show that there are many studies in which the evidence provided by cranial data appears to be be significantly incongruent with the postcranial data - more than one would expect to see just by the effect of chance and noise alone.

I devise and implement a modification to a rarely used measure of homoplasy that will hopefully encourage its wider usage. Previously it had some undesirable bias associated with the distribution of missing data in a dataset, but my modification controls for this. I also take an in-depth and extensive review of the ILD test, noting it is often misused or reported poorly, even in recent studies.

Finally, in attempting to collect data and metadata on a large scale, I uncovered inefficiencies in the research publication system that obstruct re-use of data and scientific progress. I highlight the importance of replication and reproducibility - even simple reanalysis of high profile papers can turn up some very different results. Data is highly valuable and thus it must be retained and made available for further re-use to maximize the overall return on research investment. 


\section{Chapter 1: Introduction}

Fossils provide a special 'window' through which we can glimpse the breadth and diversity of past morphological forms of life. More than $99 \%$ of all species that have ever existed are extinct (Novacek \& Wheeler 1992; Nee \& May 1997). Thus if we are to truly understand evolution we need to include extinct as well as extant forms. Through this window we have observed countless remarkable specimens of past organisms that can defy at-a-glance placement within our established schemes of classification and phylogeny for known lifeforms (e.g. Hallucigenia that was first described by Conway Morris [1977] in phylum "unknown"; see also Anomalocaris, Fig. 1). To better understand both what fossil specimens are, and to infer their relationships to other extant and extinct species, specimens are frequently compared on the basis of their morphological characteristics. These characteristics are coded into a matrix which can then be used to compare the morphological character scorings of similar organisms using numerical analyses to create a testable hypothesis of their relations - frequently expressed as a dendrogram that represents an estimate of the relations of the organisms studied.

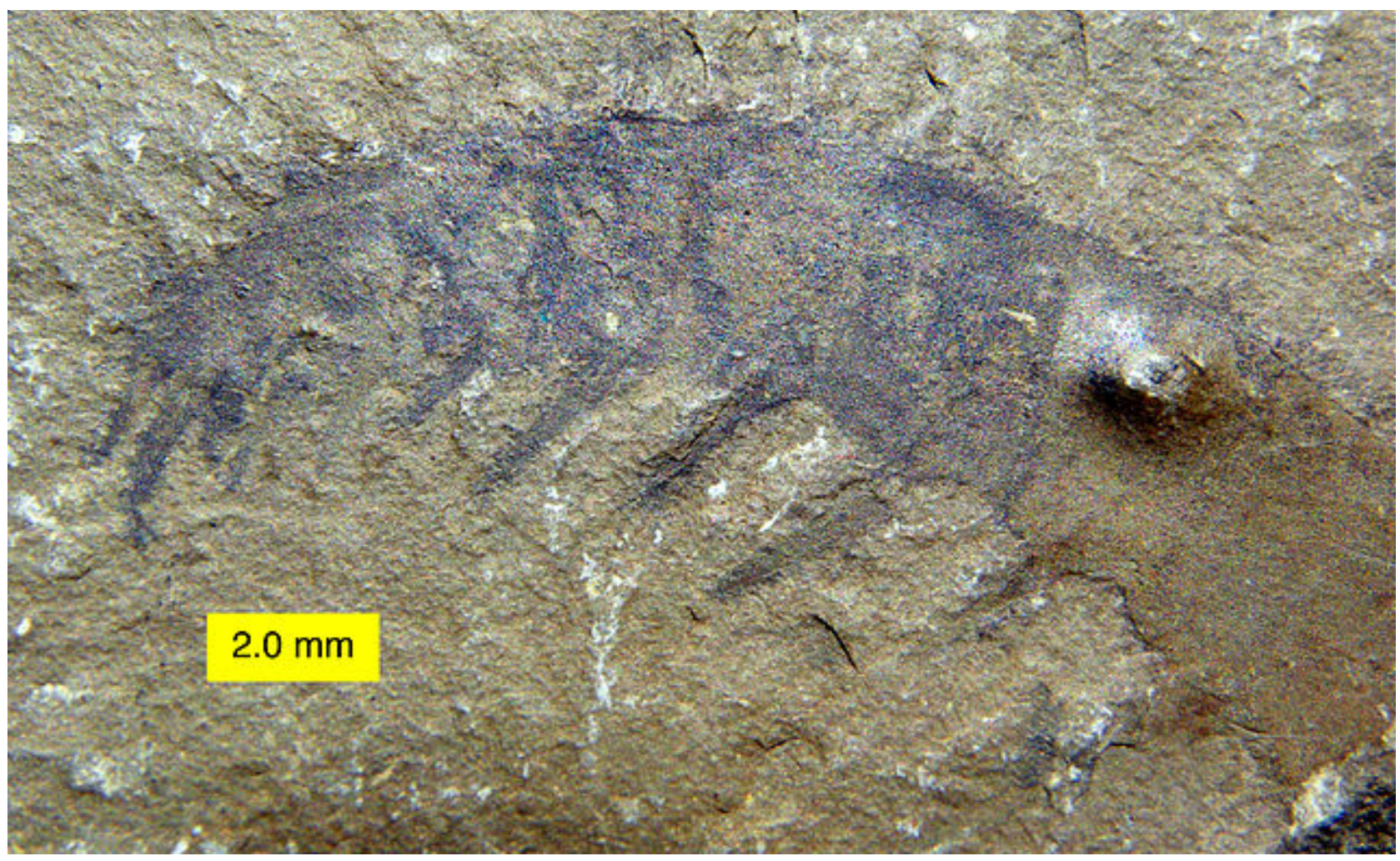

Figure 1.1 An example of a remarkable fossil form. Anomalocaris from the Mt Stephen Trilobite Beds, Middle Cambrian, near Field, British Colombia, Canada. Credit: photo by Mark A. Wilson / Public Domain. 


\subsection{Overview of Inferring Evolutionary Relationships in Palaeontology}

"There are parts of the palaeontological community where cladistic drums do not reach; there are nooks and crannies, fault lines, sink holes and caves where the words synapomorphy, paraphyly and parsimony have never been heard" Peter Forey recounting the words of his Editor (Palaeontology Newsletter), 2005

The history of classification in biological systematics can be traced back millennia to early scholars such as Aristotle, who produced one of the first recorded biological classifications. In his works such as Historia Animalium and De Historia Plantarum he grouped organisms on the basis of similarity. This system was not an evolutionary classification but it was nevertheless a noteworthy contribution. Aristotle also saw partial similarity between fossil shells and their modern counterparts but he misattributed their existence and formation as 'dry vapour exhalation' (Eichholz, 1949; Mayor, 2000). In the 18th century Linnaeus furthered the cause with his system of Linnaean taxonomy, separating nomenclature from description - a system which in essence we still use today. Linnaeus recognised three kingdoms: animal, vegetable and mineral. Although more widely-known for neontological contributions, Linnaeus also made significant contributions to palaeontology - some fossil corals still bear the same names as when he first described them after fieldwork in Gotland and elsewhere (Linnaeus, 1745; Linnaeus, 1758).

Post-Darwin, the history of classification and evolutionary relationships in palaeontology was spurred-on by George Gaylord Simpson who unlike most of his palaeontological colleagues (Olson, 1991) recognized the importance of genetics in evolutionary studies in support of the 'modern evolutionary synthesis'. Papers such as 'Patterns of phylectic evolution' (Simpson, 1937) and his opus Tempo and Mode in Evolution (Simpson, 1944) used quantitative statistical reasoning to support his arguments of fossil mammal relationships and species delimitation. His work placed palaeontology as a more objective science because of this quantitative, statistical reasoning. In the 1960's, two distinct schools of quantitative methods for inferring evolutionary relationships arose - that of numerical taxonomy and that of phylogenetic systematics (cladistics). Numerical taxonomy again worked on the basis of just similarity. Sokal \& Sneath (1963) in their book Principles of Numerical Taxonomy define numerical taxonomy as "the grouping by numerical methods of taxonomic units into taxa on the basis of their character states" (p4). Their explicit preference for similarity as the optimal criterion is made clear as early as page 6 
where they write "Similarities among taxonomic entities can be represented geometrically by points in a space... The distances between the points can be regarded as taxonomic distances". Today, few systematists, palaeontologists or otherwise use similarity as an optimality criterion for inferring phylogeny. Instead, the other major branch, phylogenetic systematics (cladistics) as it was called then and sometimes still is now, dominates as the preferred method with which to infer relationships.

These cladistic methods that we now use to infer phylogeny were first devised by a German neontologist; Willi Hennig (1950) who studied true flies (Diptera). But it wasn't until the publication of an English translation of a modified version of this book (Hennig, 1966), and various popularisers (e.g. Brundin, 1966), that his methods gained widespread recognition.

For a time after this, most of palaeontology seemed slow and even reticent to adopt these new methods on fossils. Looking back on it, commentators such as Siddall (1998) explained this resistance as attributable to "the follies of ancestor worship" - using the temporal sequence of fossils to assert ancestor-desendent relationships between fossils, rather than testing relationships of common ancestry. Hennig's 'phylogenetic systematics' (which we now refer to as cladistics) was met with "distrust" by some palaeontologists and viewed as a threat to the primacy of palaeontology's role in tracing phylogenetic pathways (Forey 2004). Prior to the Hennigian revolution, palaeontological data had been considered "both necessary and sufficient" for phylogeny reconstruction (Forey 2004), cladistics challenged this. A notable early exception to the distrust was a chapter by Schaeffer et al. (1972); three palaeontologists that clearly advocated the use of cladistic principles in palaeontology:

“...we agree that (1) the degree of relationship (as defined by recency of common ancestry) should be determined initially on the basis of morphology alone..."

Even then though, there was still a hesitance as to whether one could or should mix recent and fossil taxa in the same analysis. Among those who helped make it clear that recent \& fossil taxa could in fact be used in the same analysis together was Farris (1976):

"Fossil species - when they are sufficiently well known to be classified at all - should therefore be treated exactly as are recent species in a phylogenetic classification".

Patterson's (1981) review of the significance of fossils in determining evolutionary 
relationships represents a turning point in palaeontological thinking:

"before the development of the cladistic method paleontology was a hindrance rather than a help, stifling progress towards the goal of determining evolutionary relationships"

But in his review Patterson (1981) also peculiarly (considering he was a palaeontologist) belittles the role of fossils with his oft-cited conclusion that it is rare or unknown for fossils to overthrow theories of relationship primarily based on Recent forms (p.219). This has been convincingly refuted many times since (e.g. Gauthier et al. 1988; Donoghue et al 1989; Eernisse and Kluge 1993; Cobbett et al 2007). Patterson (1981) also noted that the incompleteness of fossils specimens relative to Recent specimens makes them inherently less informative (but as was shown later it is not what is missing that counts per se but instead what information is present that most affects phylogenetic inferences e.g. Huelsenbeck 1991; Wiens 2003a,b; Edgecombe 2010).

The schism between palaeontological and neontological approaches to phylogenetic reconstruction was so fascinating that it even drew the attention of philosophers of science. Grantham (2004) expounded at great length on this matter evaluating a number of hypotheses as to the root cause of the integration difficulty, and concludes that it is a relatively unique phenomenon relative to other fields. For a more complete history in the fuller context of systematics read Forey (2004).

\subsection{Why not just use molecular data?}

Morphology for most fossil specimens is all the evidence we have. Whilst for Recent organisms we nearly always additionally or solely use molecular sequence evidence, this cannot be obtained from most fossils for a variety of reasons (aside from issues of 'difficulty' or 'expense'). In the field of ancient DNA studies, the oldest fossil from which a genome has been extracted is a $\sim 700,000$ year old horse (Orlando et al., 2013). For the age of the oldest successfully extracted DNA fragments there is great controversy over the repeatability and validity of claims. Cano et al (1993) reported extracting DNA from an amber-entombed weevil that is 120-135 million-years-old. Many others have also claimed DNA extraction from geologically ancient fossil specimens (reviewed in Hebsgaard et al 2005). Yet subsequent attempts to repeat these feats have cast significant doubt on the validity of these claims (e.g. Austin et al 1997; Gutierrez \& Marin 1998). Moreover, wellparameterized models estimate that the half-life of mitochondrial DNA (typically better 
preserved than nuclear DNA because there is $\sim 1,000$ times more of it) is 521 years and thus even in optimal preservational conditions no mitochondrial sequence of greater than one base pair should remain after 6.8 million years (Allentoft et al 2012). Thus for fossils older than this, hard physio-biogeochemical constraints mean it is likely we will never be able to extract geologically ancient DNA samples from most fossils and hence must continue to use morphology-based methods of phylogenetic inference for the forseeable future with these taxa.

\subsection{Is Morphology Actually Useful for Reconstructing Phylogeny?}

In much of my introduction so far I have not addressed the very use of morphology itself (whether neontological or palaeontological) in reconstructing phylogeny. The use of morphology to infer phylogeny is not without its critics. Papers such as Scotland et al. (2003) have argued that fewer (but more rigorous) morphological characters should be used for phylogenetic inference. One of the points that Scotland et al (2003; hereinafter SEA ) make is that the same observed morphological characters states can often be coded in very different ways. The choice of character coding method for most morphological character states is thus subjective and the use of these different methods alone can cause real differences in the resulting phylogeny. This is not such a problem for molecular sequence data where are mostly always just 4 states, but the inference of alignment gaps and their treatment somewhat complicates this. Another point made by SEA was that the conceptualization of morphological characters themselves is also subjective.

"Different workers will perceive and define characters in different ways" (Smith 1994, p34) Simply put - using DNA sequence data is more objective than using morphological data because DNA offers large numbers of relatively unambiguous characters and character states. SEA concludes that:

"morphology is being superseded by DNA data for phylogenetic studies because much of the useful morphological diversity has already been scrutinized... We disagree that morphology offers any hope for the future to resolve phylogeny at lower or higher taxonomic levels"

Needless to say, this controversial paper provoked some very direct responses (Jenner 
2004a; Wiens 2004; Smith \& Turner 2005). Jenner (2004a) dismisses SEA's points about the subjectivity of morphological homology assessment and character coding as a "straw man" argument. In contrast Jenner (2004a) instead predicts that the application of new analytical techniques will help further and improve, refine and expand our assessments of morphology for use in phylogenetic reconstruction. The application of new 3-dimensional imaging methods to help assess morphology certainly seems to support this point (e.g. Ragsdale and Baldwin, 2010). Another important counter-point from Jenner (2004a, p 337) is that there is no evidence to suggest that morphology generally performs more poorly than molecular data in fair comparisons. Jenner (2004a) cites examples where different molecular analyses conflict with each other, and where morphology appears to be more reliably than molecular data. Clearly there is no a priori general rule as to what type of data is 'better' for phylogenetic accuracy.

Wiens (2004) makes some subtly different points to Jenner (2004a), expanding upon the importance of fossils and hence the necessity of morphology. Wiens (2004) conveys that fossils help not just in determining phylogenetic relationships, but also in our understanding of the timing and rate of evolutionary processes - such uses of 'fossil calibration' points (reviewed in Donoghue and Benton 2007) are commonplace now. Wiens (2004) points out that SEA's argument against morphology because of its frequently incomplete nature is also irrelevant. Previous studies by Wiens (2003a,b) show that highly incomplete taxa can be placed with $100 \%$ 'accuracy' relative to simulated data, and that incompleteness may limit but does not outright prevent such incomplete taxa overturning the relationships of more complete taxa. Wiens (2004) also points out that for many taxa we have very few precious specimens for them, and that we cannot extract DNA from these owing to their rarity and sometimes the way in which they have been preserved these also may require morphology-based assessment to determine phylogenetic placement. Wiens (2004) presents morphological data as a best "reality-check" for molecular studies and brushes away the criticisms presented by SEA, in that although some are problematic, the benefits of using morphology should outweigh the negatives. Wiens (2004) rejects SEA's claim that all the 'good' morphological characters have been found already and that there are few good ones left to add based on his own experience and publications. I must say that at a glance of any volume of Journal of Vertebrate Palaeontology you will clearly find studies that are finding new morphological characters, even in extremely well studied groups. High profile examples that have massively 
expanded the suite of morphological characters for well studied groups include (squamata - Conrad 2008; post-Paleozoic echinoids - Kroh \& Smith 2010; arthropods - Legg et al. 2012; placental mammals - O'Leary et al 2013).

Wiens (2004) repeatedly points to his study of congruence (Poe \& Wiens 2000) which provides empirical evidence against some of SEA's points. Wiens (2004) conclusion is that instead of abandoning morphological phylogenetics we should instead work on extending and refining our morphological characters and characters states to address some of the problems that SEA point out.

The last of the direct critiques of SEA was published a little later (Smith \& Turner, 2005). They claim that SEA misinterpreted some of the previous studies that SEA cited in support of their claims. In particular Smith \& Turner (2005) rightly point out that Hillis $(1996 ; 1998)$ used models of evolution and molecular data and that the conclusions from these analyses may not necessarily be transferable to morphological data which typically has very different properties. As Smith \& Turner (2005) are both qualified systematic palaeontologists themselves they are well placed to rebutt SEA's assertion that most new morphological characters will be more homoplastic. They rightly point out that SEA provide no evidence for this, and helpfully suggest ways in which this could be formally tested. Smith \& Turner (2005) similarly uphold the importance of fossils and morphology in phylogenetic analyses.

SEA's paper is far from the only one to criticize the very use of morphology but it is a rather obvious one on which to focus because it draws out many of the arguments for and against. Alternative philosophical approaches such as that of consilience (Wilson, 1998; Pisani, 2002) also support the use of both molecular and morphological data. I hope this short synopsis demonstrates that although questioned, the exclusion of morphology and fossils from phylogenetic analysis "is neither theoretically nor empirically defensible" (Edgecombe, 2010). 


\subsection{Aims of this thesis}

This thesis is first and foremost, a synthesis of morphology-based phylogenetic literature. In each of these chapters I perform various comparative cladistic analyses with appropriate statistical power with which to test hypotheses that one couldn't otherwise attempt to answer with just a handful of data sets. The discovery, sampling, standardisation and assembly of evidence of these chapters is more rigorous and systematic than many similar comparative cladistic analyses that have been attempted before.

Chapter 2 is an examination of the congruence of phylogenetic signal within vertebrate morphological data sets. I compare the congruence of signal between cranial and postcranial partitions of data sets to answer questions over the levels of homoplasy in each and the significance of difference between the two if any. I also explore the performance of a new statistical test of congruence of relationships inferred from data, as proposed by MAW called the Incongruence Relationship Difference (IRD) test.

Chapter 3 is a re-examination of the impact of fossil taxa in mixed phylogenetic analyses that include both extinct and extant taxa, using newer, larger data matrices. In this chapter I re-implement pre-existing methods in a new more computationally-efficient pipeline that should encourage other systematists to use these methods to explore their own data.

Chapter 4 is a critical systematic review of the usage of the ILD test in the recent literature. In this chapter I observe a number of worrying trends in the usage of the ILD test that are inappropriate and unsupported by published evidence. I hypothesise how these usages took hold in the literature and conclude with clear guidelines at the end which should hopefully ameliorate any confusion.

Chapter 5 is the introduction of a modification to Archie's (1989) Homoplasy Excess Ratio that improves the statistic when in the presence of significant amounts of non-randomly distributed missing data (as it typical of many palaeomorphological data sets). I demonstrate the improvement to estimates of homoplasy that this modification gives, and I implement it with a script in TNT so that other investigators can also use it.

Chapter 6 is a critical examination of the fragmentation, discoverability and accessibility of phylogenetic knowledge in the modern age. I compare traditional literature search engines 
such as Web of Knowledge that only search titles, abstracts and keywords, with local desktop full-text search methods over tens of thousands of papers. The findings in this chapter raise significant questions as to our ability to effectively synthesise data from thousands of papers

Chapter 7 provides my overall conclusions on what I have found with respect to the importance of fossils in phylogenetic reconstruction. But in this chapter I also take the opportunity to reflect the on some emergent themes in my thesis, namely; data availability, analysis replicatability, and how the way in which we choose to publish our research affects our ability to re-use data. 


\title{
Chapter 2:
}

\section{The Congruence of Cranial \& Postcranial Characters in Vertebrate Phylogeny}

\author{
Based on a manuscript co-authored with Matthew A. Wills
}

\subsection{Abstract}

Morphological data matrices frequently contain significantly more characters from some anatomical regions than others. Such preferential sampling is seldom considered problematic because homogeneity of phylogenetic signal across anatomical regions is almost invariably assumed. For this reason, signals within logical or anatomical partitions of morphological data sets are rarely compared. In vertebrate systematics, the cranium has often been afforded particular focus; either because it is believed to yield characters containing less homoplasy, or because morphological variation therein is more readily atomized. An analysis of 62 vertebrate data sets published between 2000 and 2010 confirms that characters of the cranium account for the significant majority, but finds equivocal evidence that they contain less homoplasy. We caution that neither partition ensemble consistency indices $(\mathrm{Cl})$ for partitions, nor mean per character consistency indices (ci) within partitions should be interpreted uncritically. Surprisingly, partition homogeneity (ILD) tests of the signal in cranial and postcranial characters reveals significant incongruence in a large minority of cases. Similarly, the trees inferred from the partitions are more different (Robinson Foulds and maximum agreement subtree distances) than expected about one time out of three (new randomization tests are proposed). This may reflect different selective pressures in particular body regions, allied with different localized patterns of homoplasy. In many cases, therefore, concentrating upon cranial characters at the expense of others (or vice versa) is quite likely to yield a phylogeny significantly different from that which would be obtained from a more holistic approach. We show that the broadest possible sampling of characters in the total evidence analysis of all aspects of morphology is most optimal, and caution against the assumption of signal homogeneity across all body regions. 


\subsection{Introduction}

Phylogenies are typically inferred from morphological data by applying maximum parsimony to all coded characters. While studies often focus upon characters of particular types or from particular organ systems, it is rare that trees are inferred explicitly from subsets of these data (but see O'Leary et al., 2003, Poyato-Ariza, 2003, Farke et al., 2011), or that the signals from non-overlapping data sets (e.g., osteology and musculature) are compared. The practice of what is effectively a default total evidence analysis for morphology contrasts with the more qualified approach often adopted with molecular data. This is partly because sequence data are readily partitioned into logically distinct classes and sub-classes: nuclear genes, mitchondrial genes (plastid genes in plants), coding and non-coding sequences as well as codon positions (Bull et al., 1993). It is widely understood that the signals from different types of molecular data (or indeed, from any two loci) can conflict (Felsenstein, 1988; Pamilo \& Nei, 1988; Maddison, 1997; Nichols, 2001). This has been referred to as the 'gene tree discordance' problem (Degnan \& Rosenberg, 2009), although we note that such discordance is not necessarily a result of different gene histories. In trees derived from modest numbers of molecular markers, signals may be variously tested for homogeneity prior to combination. At the other extreme, where the products of next-generation sequencing are assembled for phylogenomic analysis, it is common to apply filters of varying complexity to ensure a signal of some specified quality or homogeneity (Leigh et al., 2011; von Reumont et al., 2012).

Incongruence between partitions of molecular data can be attributed to a variety of causes: non-vertical inheritance affecting one or both partitions (e.g., gene loss/duplication, horizontal transfer, hybridisation or recombination), deep genetic divergence that does not reflect species phylogeny (also known as 'hemiplasy' or 'incomplete lineage sorting'; Avise \& Robinson, 2008; Degnan \& Rosenberg, 2009), or to a difference in the rate of evolution between partitions (Planet, 2006). Incongruence is also commonly observed between morphological and molecular data partitions (e.g., Mickevich \& Farris, 1981; Bremer, 1996; Poe, 1996; Baker et al., 1998; Jenner, 2004b; Draper et al., 2007; Springer et al., 2007; Near 2009). Occasionally, this is attributed uncritically and a priori to a spurious morphological tree, with little specific justification (e.g., Hedges \& Sibley, 1994; Hedges \& Maxson, 1996; D'Erchia et al., 1996). More often, however, authors acknowledge the value of both classes of data, and cede that incongruence may 
actually be informative. In all but the most exceptional circumstances (such as in artificial selection experiments or where cladogenesis has occurred in recorded history) one can never be sure that any analytical result matches the 'correct' species tree. Moreover, perfectly bifurcating cladograms may be an oversimplification of actual evolutionary history (Doolittle \& Bapteste, 2007; Lopez \& Bapteste, 2009; Ragan et al., 2009).

\subsubsection{Congruence between Partitions of Morphological Data}

While the congruence of signals from different loci is routinely investigated for molecular sequence data, morphological data sets are rarely partitioned explicitly, still less subjected to tests of congruence (although see Smith, 2010; Clarke, 2011; Bennett, 2013 for some recent exceptions). Homogeneity of the morphological signal has often been tacitly assumed (Mayr, 1953; Michener, 1953): the 'hypothesis of nonspecificity' (Sokal \& Sneath, 1963). Early attempts to test this assumption applied phenetic methods to data sets of modest dimensions, and yielded equivocal results. Sokal and Sneath (1963), who addressed the issue in the context of vertebrate systematics, stressed the similarity between dendrograms from different sources and concluded that nonspecificity was the rule. Farris (1971), by contrast, emphasised the detailed differences in such cases. If anything, the ascendancy of cladism (sensu Hennig, 1966) appears to have further relegated the issue of character congruence for morphological partitions: only a handful of studies have addressed the issue directly (e.g., Sánchez-Villagra \& Williams, 1998; Gould, 2001; Song \& Bucheli, 2010). With the rise of molecular data, the issue of morphological versus molecular incongruence has been much more to the fore (Kluge, 1989), possibly motivated by striking examples of conflict between molecular and morphological cladograms in some groups (Bledsoe \& Raikow, 1990; Hillis \& Wiens, 2000; Wiens \& Hollingsworth, 2000; Pisani et al., 2007; Mayr, 2011a). In the face of these apparently much more invidious difficulties, seeking nuances of signal variation within (potential) partitions of the morphological data has been a low priority. Historically, the focus of research also shifted to taxonomic congruence (sensu Mickevich, 1978; Miyamoto \& Fitch, 1995) (differences in the implied relationships of taxa on alternative trees), rather than character congruence.

When cladograms were predominantly generated manually, the processes of formulating and coding characters were intimately associated with those of tree 
construction (Hull, 1990). Practical considerations limited the number of characters that could be analysed, and putative characters were iteratively tested against one another and the coalescing phylogeny (Kitching et al. 1998). This almost certainly had the effect of screening out 'noisier' characters, or those contributing greater amounts of homoplasy to the dataset. To the extent that operational definitions of homology are ultimately predicated on the distributions of other characters, it might be considered that this effectively made assessments of probable homology more stringent. With the appearance of faster computer hardware and parsimony programs (Farris et al., 1970), there were less computational restrictions on the size of datasets that could be processed and the complexity of character conflicts that could be resolved. Mooi \& Gill (2010) argue that this, coupled with the desire to increase the ratio of characters to taxa, may have encouraged practitioners to incorporate as many characters as possible, with more relaxed 'quality control'. At the same time, there is no imperative for authors to explore the congruence of their characters a priori (sensu Grant \& Kluge, 2003), because computer algorithms will resolve the conflicts, however 'noisy'. Cladistic analyses therefore have two temporally and logically distinct phases (Winther, 2009): character analysis (or the determination of homology: Pinna, 1991), and phylogenetic analysis. Mooi \& Gill $(2010$, p1) contend that current practices are heavily skewed towards the latter, relying too heavily on "algorithms and statistics rather than biology to determine relationships". We do not interpret this as an indictment of the value of methodological advances, but rather as highlighting the comparable dearth of work on evaluating homology prior to analysis.

The concept of 'character congruence' is akin to Sneath \& Sokal's (1963) 'nonspecificity', but in an expressly cladistic context. Characters are congruent if their character state trees (sensu Estabrook, 1968) are compatible (Le Quesne, 1969), in much the same way that entire cladograms are congruent in the absence of conflicting nodes (Wheeler, 1981).

\subsubsection{Previous Quantitative Studies}

There is an extensive and mature literature on the use of morphological characters to infer the phylogeny of vertebrate groups, with studies having burgeoned in parallel with the development of modern cladistic methods (Hennig, 1950; Ashlock, 1974). Historically, the focus has been on osteological characters, but morphology also includes non-osteological 
'soft part' characters such as those from the integument, internal organs, musculature, reproductive organs, gametes, tissue and cellular structures. Many other characters that are not observable morphologically are nonetheless grouped with them in most character lists (ostensibly because they are not conventional nucleotide or amino acid sequence data). These include karyotypes (Faivovich, 2002), behavioural (Lee \& Scanlon, 2002; Faivovich, 2002; Hill, 2005; Li et al., 2007; Spaulding et al., 2009), and ontogenetic sequence (Seiffert, 2007; Simmons et al., 2008) characters. Despite the increasing importance of molecular and genomic data over the last two decades, morphology still makes an invaluable contribution to vertebrate phylogenetics, and is indispensible for the analysis of fossil species. Levels of morphological homoplasy in vertebrate groups are generally lower than those amongst their invertebrate counterparts (Hoyal Cuthill et al. 2010), suggesting that the signal quality for vertebrates is relatively high. Moreover, the inferred relationships within many vertebrate clades have altered little with the progression of research time or with the addition of molecular data (albeit with some spectacular exceptions: e.g., Asher et al. 2009).

Although cladists occasionally publish variations of trees derived from subsets of their character data (e.g., O'Leary et al., 2003; Poyato-Ariza, 2003; Diogo, 2004 p417-429; Young, 2005; Farke et al., 2011), very few studies have investigated the performance of character partitions quantitatively and systematically using homoplasy indices. The consistency index $(\mathrm{Cl})$ is calculated as the ratio of the minimum number of steps a character can exhibit on any cladogram to the minimum number of steps the same character can exhibit on the cladogram in question. The retention index $(\mathrm{RI})$ is a measure of the level of synapomorphy of characters on a given cladogram. A handful of studies have previously compared distributions of consistency (Kluge \& Farris, 1969) and retention (Farris, 1989a) indices across characters in two or more partitions. Most recently, Song \& Bucheli (2010) inferred these indices for male genital and non-genital characters in insect systematics, finding genital characters to be statistically less homoplasious. Similarly, two other studies compared internal anatomical and external shell character partitions for brachiopods (Leighton \& Maples, 2002) and gastropods (Vermeij and Carlson, 2000). The latter paper revealed that shell characters were significantly more homoplastic than internal anatomical characters: a disconcerting finding in a group whose fossils are studied almost exclusively with recourse to hard part characters. In a study of hedgehogs, Gould (2001) reported significantly higher consistency indices for dental characters compared with the remainder of his dataset. However, he also found that the optimal trees inferred 
from the dental characters alone were seriously at odds with those implied by the other characters, and he cautioned against the uncritical use of the former. Most ambitiously, Sanchez-Villagra and Williams (1998) compared the consistency indices between dental, cranial and postcranial character partitions of eight mammalian data sets. These were of modest proportions (an average of eleven taxa), but they reported no significant differences for any of their comparisons. Unfortunately, this general approach is complicated by differences in the sizes of the dataset partitions, coupled with the inverse correlation between both consistency and retention indices and the number of taxa (Archie, 1989; Sanderson \& Donoghue, 1989; Klassen et al., 1991; this paper). We therefore adopt a variety of allied methods in this study.

\subsubsection{Why Examine the Congruence of Cranial and Postcranial Partitions?}

Character analysis is often the least well explored aspect of systematic analyses (Pogue \& Mickevich, 1990). Amongst vertebrate systematists, it is often asserted that cranial and postcranial characters convey signals of differing quality (Ward, 1997; Collard et al., 2001; Naylor \& Adams, 2001; Finarelli \& Clyde, 2004). However, the evidence for this is piecemeal and largely anecdotal, with few attempts to quantify putative differences. Many practitioners take a more or less even-handed approach to sampling characters (SánchezVillagra \& Williams, 1998), attempting to avoid describing a disproportionate number from any one anatomical region (Sokal \& Sneath, 1963). However, even where potential characters are reasonably homogeneously distributed throughout the body, "certain body regions and organs still hold a considerable mystique for taxonomists as classificatory tools, while others are neglected" (Sokal \& Sneath, 1963; page 85). For example, Arratia (2009) notes that actinopterygian systematists focus their attention significantly toward cranial characters, despite rich seams of underexploited data within the fin rays and fulcra. In this study, we apply a variety of methods to explore differences in the strength and character of phylogenetic signals in cranial and postcranial partitions of 62 published vertebrate data sets.

We address the following questions: 1 . Is there a significant difference between the number of cranial and postcranial characters in our sampled data sets? Received wisdom 
holds that the cranium is a richer source of phylogenetic data that the postcranium (Sanchez-Villagra \& Williams, 1998). 2. Are levels of homoplasy in cranial character partitions lower than in postcranial character partitions (Sanchez-Villagra \& Williams, 1998), and are any observed differences more than simply a function of differing numbers of characters within these partitions? 3. Is there more conflict between cranial and postcranial characters than we would expect, and are the relationships inferred from them less similar than we might predict?

\subsection{MATERIALS AND METHOdS}

Phylogenetic data sets published between 2000 ad 2010 were sourced from the peerreviewed literature. We restricted our focus to discrete character morphological matrices composed entirely of vertebrate taxa, and analysed using equal weights maximum parsimony. Matrices were initially garnered from Brian O'Meara's TreeBASE mirror (O'Meara, 2009), Graeme Lloyd's online collection of non-avian dinosaur matrices (Lloyd, 2009), MorphoBank, (O'Leary \& Kaufman, 2011) directly from authors, or from the original papers. We then filtered these by removing matrices with no cranial or postcranial characters, in addition to those with fewer then eight taxa or partitions with fewer than eight parsimony-informative characters (for reasons of statistical power). We interpret cranial characters here as those pertaining to the skull (cranium plus mandible and dentition) rather than as just those of the cranium. In cases of taxonomic overlap (or where one matrix was derived from an earlier one), we retained only the most inclusive (usually the most recent) dataset if the fraction of terminals in common was $50 \%$ or more of the size of the smaller matrix. A modest number of matrices were also excluded because they contained more than 200 taxa or more than 1,000 characters (e.g., for Squamata; Conrad, 2008 and derivative papers). These were highly atypical, and could not have been analysed with comparable rigour in a tractable time with our methods.

Our resulting sample comprised 62 matrices, spanning all major vertebrate groups, and sampled at a variety of taxonomic levels. A minority of these data sets contained a small number of characters that were not strictly morphological (e.g., character 618 relating to habitat choice in the matrix of Spaulding et al., 2009). These were removed prior to any further analysis. We also removed phylogenetically uninformative taxa within partitions using the principles of safe taxonomic reduction implemented in TAXEQ3 (Wilkinson, 1995b). Additionally, parsimony-uninformative characters were removed 
because they have undesirable effects on many of the tests that we subsequently performed (Carpenter, 1988; Sanderson \& Donoghue, 1989; Bryant, 1995; Lee, 2001b). The number and percentage of cranial and postcranial characters in each matrix were recorded. Wilcoxon signed-rank tests were used to assess the significance of differences between these means in different groups.

\subsubsection{Do Cranial and Postcranial Characters Imply Different Levels of Homoplasy?}

Consistency and retention index comparisons. - All phylogenetic analyses were performed using PAUP* 4.0b10 (Swofford, 2002), using equally weighted parsimony analysis. We also reproduced any preferred assumptions concerning character order, as well as assumptions concerning character polarity and rooting. Empirically, we determined that 20 random addition sequence replicates followed by TBR branch swapping holding up to 1000 trees at each cycle (and limited to retaining a maximum of 10,000 trees overall) were effective at recovering the set of MPTs reported by the original authors in each case. We therefore used these settings for all subsequent analyses. Such heuristic searches were a necessary compromise given constraints of computational time and memory. Cranial and postcranial character partitions were specified using charset commands. There are two obvious ways in which to calculate differences in mean/median consistency indices (ci; Kluge \& Farris, 1969) and retention indices (ri; Farris, 1989) for characters in partitions of a dataset, but there are drawbacks to both. The first (and usual) approach is to find the optimal tree or trees for all characters simultaneously (the global MPT(s) on the principle of total evidence; Kluge, 1989) and to take mean values for characters reconstructed on this/these (Sánchez-Villagra \& Williams, 1998; Song \& Bucheli, 2010). Where characters in the two partitions contain different levels of homoplasy but support the same tree(s), this approach is relatively straightforward. However, the situation is more complex when the partitions support different trees, and especially when these partitions are also of different sizes. In this case, with no differences in the levels of character conflict within partitions (and all other things being equal), the larger partition is more likely to determine the overall pattern of relationships. 
a

\begin{tabular}{llll} 
Taxon & \multicolumn{3}{c}{ Characters } \\
\hline & 1 & 2 & 3 \\
\hline \hline Outg. & 0 & 0 & 0 \\
A & 1 & 1 & 1 \\
B & 1 & 1 & 1 \\
C & 0 & 1 & 1 \\
D & 0 & 0 & 1 \\
E & 0 & 0 & 0
\end{tabular}
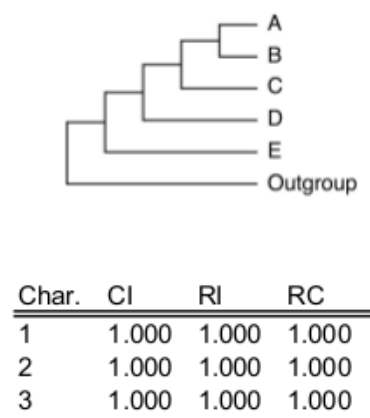

b

\begin{tabular}{llll} 
Taxon & \multicolumn{3}{c}{ Characters } \\
\hline & 4 & 5 & 6 \\
\hline \hline Outg. & 0 & 0 & 0 \\
A & 0 & 0 & 0 \\
B & 0 & 0 & 1 \\
C & 0 & 1 & 1 \\
D & 1 & 1 & 1 \\
E & 1 & 1 & 1
\end{tabular}

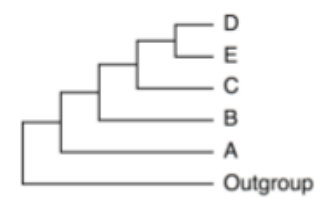

\begin{tabular}{llll} 
Char. & Cl & RI & RC \\
\hline \hline 4 & 1.000 & 1.000 & 1.000 \\
5 & 1.000 & 1.000 & 1.000 \\
6 & 1.000 & 1.000 & 1.000
\end{tabular}

C

\begin{tabular}{lllllll} 
Taxon & \multicolumn{7}{c}{ Characters } \\
\hline & 1 & 2 & 3 & 4 & 5 & 6 \\
\hline \hline Outg. & 0 & 0 & 0 & 0 & 0 & 0 \\
A & 1 & 1 & 1 & 0 & 0 & 0 \\
B & 1 & 1 & 1 & 0 & 0 & 1 \\
C & 0 & 1 & 1 & 0 & 1 & 1 \\
D & 0 & 0 & 1 & 1 & 1 & 1 \\
E & 0 & 0 & 0 & 1 & 1 & 1
\end{tabular}

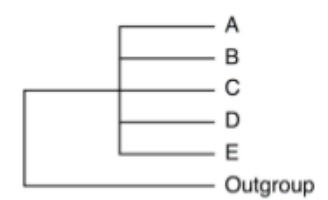

\begin{tabular}{llll} 
Char. & $\mathrm{Cl}$ & $\mathrm{RI}$ & $\mathrm{RC}$ \\
\hline \hline 1 & 0.875 & 0.750 & 0.750 \\
2 & 0.750 & 0.750 & 0.625 \\
3 & 0.625 & 0.250 & 0.250 \\
4 & 0.875 & 0.750 & 0.750 \\
5 & 0.750 & 0.750 & 0.625 \\
6 & 0.625 & 0.250 & 0.250
\end{tabular}

d

\begin{tabular}{lllllllll} 
Taxon & \multicolumn{10}{c}{ Characters } \\
\hline & 1 & 2 & 3 & 4 & 5 & 6 & $5^{\prime}$ & $6^{\prime}$ \\
\hline \hline Outg. & 0 & 0 & 0 & 0 & 0 & 0 & 0 & 0 \\
A & 1 & 1 & 1 & 0 & 0 & 0 & 0 & 0 \\
B & 1 & 1 & 1 & 0 & 0 & 1 & 0 & 1 \\
C & 0 & 1 & 1 & 0 & 1 & 1 & 1 & 1 \\
D & 0 & 0 & 1 & 1 & 1 & 1 & 1 & 1 \\
E & 0 & 0 & 0 & 1 & 1 & 1 & 1 & 1
\end{tabular}

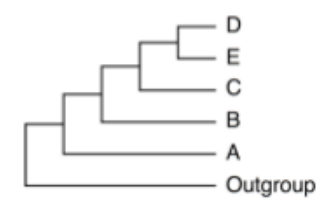

\begin{tabular}{llll} 
Char. & $\mathrm{Cl}$ & $\mathrm{RI}$ & $\mathrm{RC}$ \\
\hline \hline 1 & 0.500 & 0.000 & 0.000 \\
2 & 0.500 & 0.500 & 0.250 \\
3 & 0.500 & 0.000 & 0.000 \\
4 & 1.000 & 1.000 & 1.000 \\
5 & 1.000 & 1.000 & 1.000 \\
6 & 1.000 & 1.000 & 1.000 \\
$5^{\prime}$ & 1.000 & 1.000 & 1.000 \\
$6^{\prime}$ & 1.000 & 1.000 & 1.000
\end{tabular}

Figure $2.1 \mathrm{a} \& \mathrm{~b}$ ) A theoretical example. Characters sampled from different anatomical regions can yield radically different most parsimonious trees (MPT) when analysed in isolation. In both cases, there is no homoplasy within either region (characters 1-3 or characters 4-6), and a single MPT results in each case. c) Combining the data from both partitions (characters 1-6) yields four MPTs, the strict consensus of which (illustrated) is entirely unresolved. Character statistics have been averaged over the four trees. d) Two additional characters (5' and 6') are sampled from the same region as ' $b$ ', and these have the same distribution as 5 and 6 respectively. Analysis of all characters now reveals a single MPT with relationships identical to those in ' $b$ ' (characters 4-6). Characters 46,5 ' and 6' contain no homoplasy: all conflicts are resolved with a cost to characters 1-3. In this case, the MPT is identical to the result that would be obtained by a clique analysis (sensu Le Quesne 1969).

This also means that the characters in the larger partition are likely to have higher ci values on average (Fig. 2.1). Notwithstanding, evaluating and resolving differences in numbers of characters supporting incompatible hypotheses is an integral part of total evidence analysis (and of parsimony in general). However, another part of the rationale is to combine all available data, or at least to sample in an unbiased manner from the universe of possible characters. Because systematists tend to concentrate on particular body regions, there is the potential for self-reinforcement: the undersampled characters will actually have lower ci values upon inspection (because other body regions dominate the overall phylogenetic signal), and therefore may be less likely to receive attention in future analyses. Mean partition ci calculated in this way therefore has the potential to 
reflect sampling intensity. The second approach to measuring homoplasy is to investigate the performance of the characters within each partition analysed independently. While this will reveal information about homoplasy within partitions (rather than in one partition relative to the entire sample), the ensemble $\mathrm{Cl}$ and ensemble $\mathrm{RI}$ (and therefore ci and ri for individual characters) are known to be influenced by data set dimensions (Archie \& Felsenstein, 1993; Archie, 1996). This effect is particularly pronounced for the number of taxa, where the correlation is strong and negative. This is not a problem for comparisons within data sets (as here), because the number of taxa is always constant. However, the number of characters also has a much less marked but negative impact: one character cannot conflict with itself, while two are less likely to conflict than twenty (Fig. 2.2). This bias (for $\mathrm{Cl}$ ) operates in the opposite direction to that observed above (for mean ci in a global optimization). 

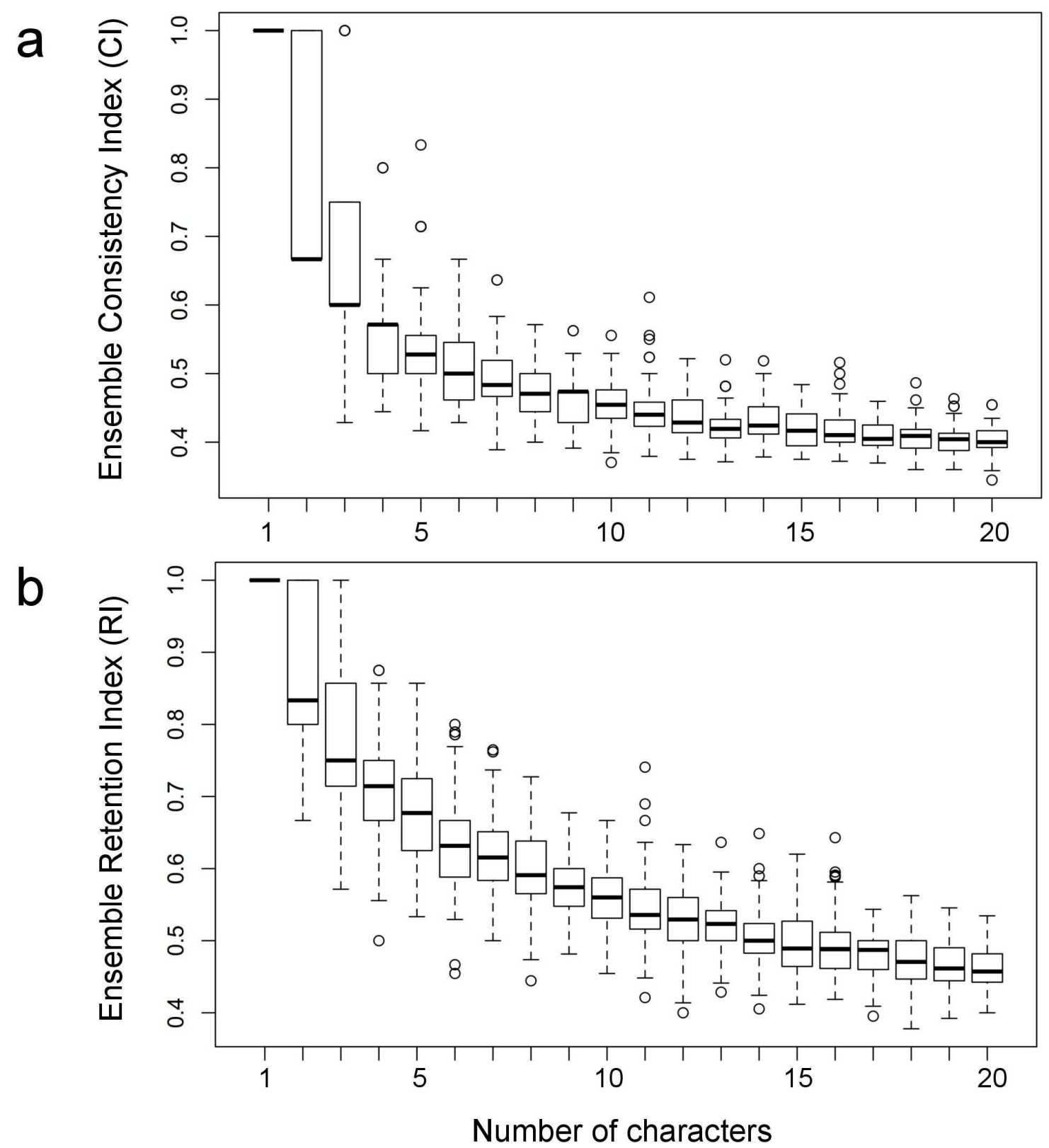

Figure 2.2 The number of characters in a data matrix influences probable indices of homoplasy. a) The ensemble Consistency Index (CI). b) The ensemble Retention Index (RI). Simulations for the trivial case of 10 taxa, with between 1 and 20 characters. All states were randomly assigned 0 or 1 with equal probability. Box and whisker plots summarise 100 replications for each number of characters. 
There are three ways in which differences between these indices can be tested. For individual data matrices, Mann-Whitney or t-tests can be applied to character ci and ri values, with the null that these have a similar median or mean in the two partitions. For the more general comparison across all 62 matrices simultaneously, Wilcoxon signed ranks or paired t-tests can be used to test the nulls that (either) the median/mean ci or ri in cranial and postcranial partitions were similar, or that the median/mean $\mathrm{Cl}$ and $\mathrm{RI}$ indices for the two partitions were similar. As discussed above, however, we note that all of these outcomes are differently and undesirably influenced by partition size.

We also note the potential for a test based on the distributions of values obtained from bootstrapped samples, controlling for differences in character number by repeatedly subsampling both/all partitions at the size of the smallest. We have not implemented such a test here.

Homoplasy Excess Ratio (HER) indices. - The homoplasy excess ratio (HER; Archie \& Felsenstein, 1993) was proposed as an adjunct to the ensemble consistency index $(\mathrm{Cl})$, and argued to be relatively immune to its worst shortcomings. Central to the calculation of the index is a randomisation procedure that operates by repeatedly permuting the assignment of character states within characters but across taxa, thereby disrupting any phylogenetic signal. A large number of randomised matrices are then analysed under maximum parsimony in order to obtain a distribution of tree lengths. This is similar to the procedure implemented by the permutation tail probability test (PTP; Faith \& Cranston, 1991). The limitations of the latter test have been rehearsed at length elsewhere (Wills 1999). However, the HER differs fundamentally from the PTP, because it does not use this distribution as the means to test a null. Rather, the mean value for randomised matrices (MEANNS) is used as an estimate of the expected tree length for matrices of the same dimensions and with identical frequency distributions of states as the original. The HER is then calculated as:

$$
\text { HER }=(\text { MEANNS }- \text { L) } /(\text { MEANNS }- \text { MINL })
$$

where $L$ is the optimal length of the original dataset and, MINL is the minimum possible length of the dataset. MINL was calculated as the total number of character states in the entire matrix, minus the number of characters. MEANNS was estimated in PAUP* using the permute command with 999 replicates and the search parameters used on the original dataset. 
The HER is calculated as a single value for a given block of data: there is no formally-proposed analogue of the index for individual characters (as the ci is a an analogue of the $\mathrm{Cl}$ ). We note that such a test would be possible by permuting the state assignments for individual characters, but we do not explore this here. The HER has therefore been calculated for the cranial and postcranial halves of each matrix when analysed in isolation. The properties of the HER mean that differences in the sizes of partitions are largely controlled for. We then tested for differences in partitions across all 62 data sets using the Wilcoxon signed ranks test.

\subsubsection{Is there More Conflict Between Cranial and Postcranial Characters than we Might Expect?}

Incongruence Length Difference (ILD) test. - To assess the significance of congruence between whole character partitions as measured by optimal tree length, the ILD test (Mickevich \& Farris, 1981; Farris et al., 1995a; Farris et al., 1995b; Barker and Lutzoni, 2002) was applied to the matrices in $P A U P^{*}$ using the hompart command, with 999 replicates (Allard et al., 1999a,b). Heuristic search settings were specified using tree bisection-reconnection (TBR), 10 random addition sequence replicates, holding up to 1000 trees at each cycle, limited to holding a maximum of 10,000 trees overall. These tests were run on a high performance computing cluster (Bioportal; Kumar et al., 2009).

The ILD score is given by $L_{A B}-\left(L_{A}+L_{B}\right) / L_{A B}$ where $L_{A B}$ is the optimal tree length (in steps) of the simultaneous analysis of both partitions together (the total evidence analysis). $L_{A}$ is the optimal tree length of an analysis of just partition $A$, and likewise $L_{B}$ is the optimal tree length of an analysis of just partition B (Fig. 3). To determine the significance of the observed ILD score, random partitions of the same size (number of characters) as the specified partitions are also generated to yield a distribution of randomized ILD scores. Thus, the ILD test is a randomization test (sensu Kempthorne, 1952), that compares the significance of a particular score relative to the scores of a set of randomly permuted replicates of the same dataset. Given the nature of phylogenetic data, the suitability of this test has been questioned on a variety of grounds (Dolphin et al. 2000; Hipp et al. 2004; Ramirez 2006; reviewed in Planet 2006). Despite this, the ILD test remains commonly used to compare the congruence of data partitions). We did not apply the arcsine 
transformation suggested by Quicke et al. (2007) because they justified their correction on the basis of empirical and simulated molecular data, whilst here we use morphological data which has different statistical properties. Morphological data matrices are mostly composed of binary or three-state characters and contain ordered characters, whilst molecular data is typically of four-state characters (e.g. GTCA) and these are never ordered in linear sequence between states. To investigate the robustness of the ILD test to taxonomic sampling, we also performed first-order taxon jack-knife ILD tests (cf. Planet \& Sarkar, 2005, but not using their scripts) on a selection of the smaller taxon data sets. This enabled global partition incongruence (all or most taxa) to be distinguished from local incongruence (caused by individually highly incongruent taxa).

Cranial partition

\begin{tabular}{llllllllll} 
Taxon & \multicolumn{10}{c}{ Cranial characters } \\
\hline & 1 & 2 & 3 & 4 & 5 & 6 & 7 & 8 & 9 \\
\hline \hline Outg. & 0 & 0 & 0 & 0 & 0 & 0 & 0 & 0 & 0 \\
A & 0 & 0 & 1 & 1 & 1 & 0 & 0 & 1 & 1 \\
B & 0 & 0 & 1 & 1 & 1 & 0 & 0 & 0 & 0 \\
C & 0 & 0 & 1 & 1 & 0 & 0 & 0 & 1 & 1 \\
D & 1 & 1 & 0 & 0 & 0 & 0 & 0 & 0 & 0 \\
E & 1 & 1 & 0 & 0 & 0 & 1 & 1 & 0 & 1 \\
F & 1 & 1 & 0 & 0 & 0 & 1 & 1 & 0 & 0 \\
\end{tabular}

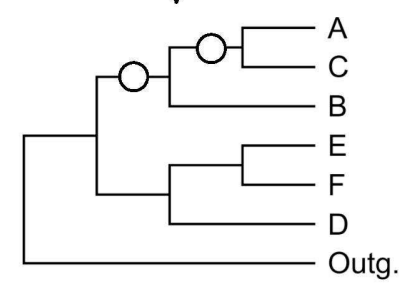

Tree from 9 cranial characters

11 steps

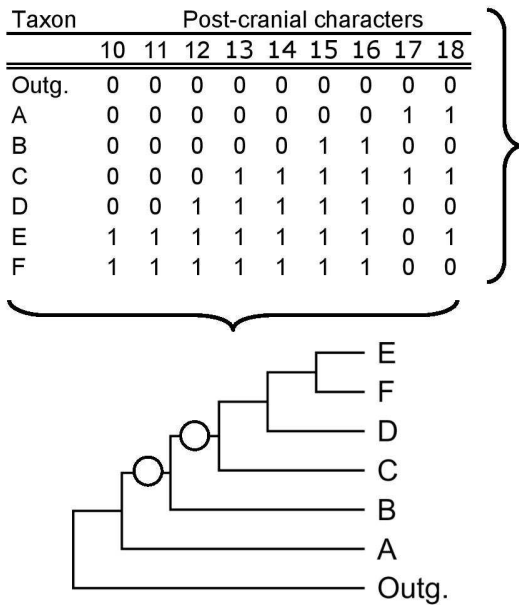

Tree from 9 postcranial characters

12 steps $=$ sum of partition lengths $=23$ steps

2 unique branches

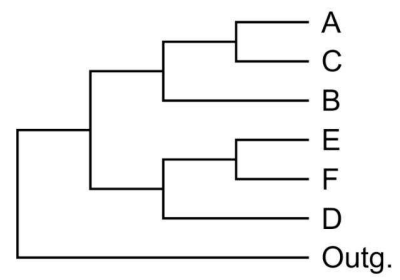

Tree from all 18 characters

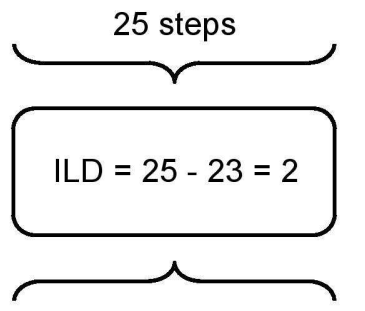

$=\mathrm{IRD}_{\mathrm{RF}}=2+2=4$

Figure 2.3 Calculation of two inhomogeneity metrics for 'cranial' and 'postcranial' partitions of a hypothetical data set. In this example there are equal numbers of cranial (1-9) and postcranial (1018) characters, but this need not be the case. For the Incongruence Length Difference (ILD) measure, maximally parsimonious trees (MPTs) are inferred from the cranial and postcranial partitions of the data independently. The summed lengths of these trees (11 steps +12 steps $)$ is the sum of partition lengths (23 steps). In parallel with this, an MPT is inferred from both partitions analysed simultaneously. This tree is longer (25 steps) than the sum of partition lengths (23 steps), and the difference between them is the ILD $(25-23=2)$. The ILD represents the reduction in homoplasy afforded by the isolation of the two partitions (two extra steps are needed when the partitions are combined). For the Incongruence Relationship Difference (IRD) measure, the 
branching structure of the cranial and postcranial partition MPTs are compared (rather than their lengths) using one of several possible tree-to-tree distance metrics. Here, we illustrate the symmetric difference distance (RF) of Robinson and Foulds (1981) (so the metric is the IRDRF). Open circles mark branches in either the cranial or postcranial MPT that are absent from the other. The tally of these unique branches on both trees is the RF $(2+2=4)$. Some background level of ILD or IRD is anticipated wherever a data set contains homoplasy. In order to interpret these observed metrics, therefore, we need to know what values would be expected for partitions of similar data sets in similar proportions. Random character partitions are used to generate null distributions for both the ILD and IRD, and observed valued deemed significantly different from the null if they lie in some specified fraction of the tails.

\section{Do Cranial and Postcranial Characters Support Different Trees?}

Templeton, winning sites and Kishino-Hasegawa tests. - All of these tests can be used to assess whether a given matrix offers significantly more support for one tree compared with another. In order to interpret the statistics that they generate straightforwardly, the alternative trees should be specified a priori rather than from an analysis of the matrix. In many applications, however, it is common to compare an optimal tree with an alternative to determine whether the latter is significantly worse. In this context, the trees inferred from the cranial and postcranial partitions were the suboptimal alternatives to MPTs from the postcranial and cranial partitions respectively. We also tested trees from individual partitions against the entire dataset. We note that these applications of the tests may be problematic (with a high type I error rate) because we are comparing an optimal with a suboptimal tree by definition (rather than two alternative trees derived independently from our data) (Goldman et al., 2000).

The Templeton test operates by calculating the length of each character on both the optimal and the alternative tree (Templeton, 1983). These paired values are then subjected to a one-tailed Wilcoxon test (Siegel \& Castellan, 1988). A one-tailed test was used because although the steps contributed by an individual character can be fewer on the suboptimal than the optimal tree, the contributions summed over all characters can only be greater. The winning-sites test (Prager \& Wilson, 1988) is very similar to the Templeton test, except that it ignores the magnitude of differences and uses only counts of 'winningsites' (parsimony-informative characters that fit more parsimoniously on one tree-topology than the other). These two sums were then analysed with a one-tailed binomial test. 
Finally, the Kishino-Hasegawa $(\mathrm{KH})$ test (Kishino \& Hasegawa, 1989; more specifically 'test priNPncs' according to Goldman et al. 2000, p658) also computes site-wise differences between trees, but makes the additional assumption that differences between sites are normally distributed. These site-wise differences of fit were then tested with a one-tailed, paired t-test: as such the $\mathrm{KH}$ test is effectively a parametric analogue of the Templeton test.

The KH test has a related family of variants (Swofford et al., 1996; Shimodaira \& Hasegawa, 1999; Buckley et al., 2001; Shimodaira, 2002) commonly used to compare trees generated in a Maximum Likelihood framework. The Shimodaira-Hasegawa test is designed for the particular case where an optimal tree is compared with a sub-optimal alternative. Although this can be applied in a parsimony context (Near et al., 2003) we are not aware of a straightforward implementation.

In practice, many partitioned and entire data sets yield more than one MPT. Where numbers are small, it would be feasible to test all alternatives. However, in many cases the numbers preclude this. We have therefore used majority rule consensus trees (including only compatible groupings with greater than $50 \%$ support). We are aware of the limitations of this approach; specifically that majority rule consensus trees need not lie at the centre of the 'tree spaces' defined by their fundamentals (see Fig. 2.3). We also note that when applied to comparisons of trees from partitions versus those from entire data sets (and all other things being equal) the 'entire' tree is more likely to be similar to that from the partition with the greater number of characters. All tests were implemented in PAUP* (Swofford, 2002).

Topological Incongruence Length Difference (TILD). - The TILD test (Wheeler, 1999) operates in a manner analogous to the ILD test, but is applied to a matrix representation of the branching structure of the optimal trees from the data partitions (rather than to the partitioned character data itself). Cranial and postcranial partitions are analysed independently, and a majority-rule ( $>50 \%$ ) consensus tree is generated for each of them. Each consensus is then translated into a matrix of group inclusion characters (GIC; Farris, 1973; also known as MRP coding, Baum, 1992; Ragan, 1992) that convey the same information as the cladogram branching structure. The GIC matrices for the partitioned analyses $A$ and $B$ are re-combined and subjected to a conventional ILD test. 
The Incongruence Relationship Difference test (IRD); a new test of the congruence of relationships (Wills pers. comm)

Cranial characters

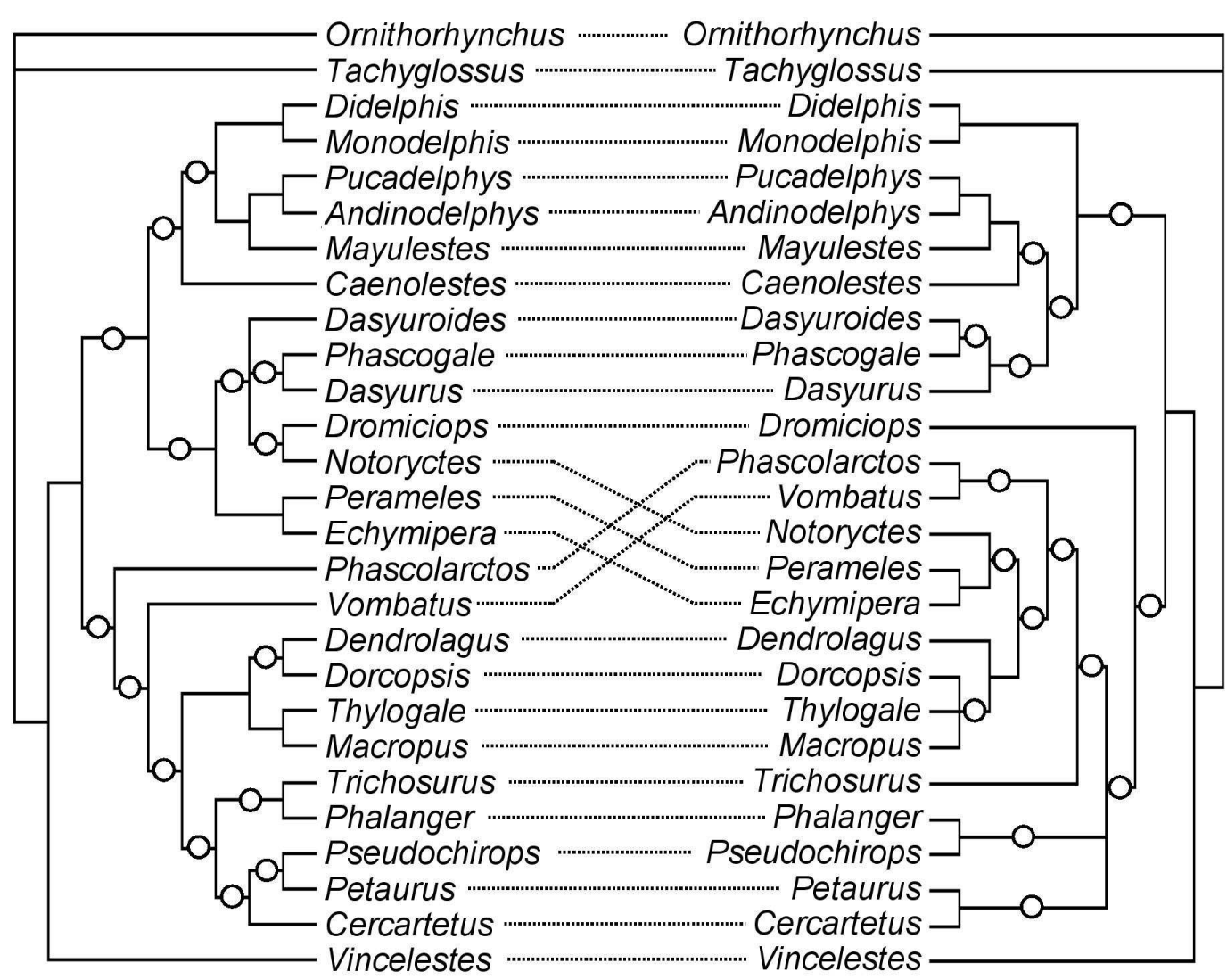

Figure 2.4 Most parsimonious trees derived from the 103 cranial and 139 postcranial characters in the mammalian data of Beck (2008). Four cranial and eight postcranial trees are summarized here as majority rule consensuses. Nodes labeled with circles are unique to one or other tree (those unlabelled are common to both). The Robinson Folds (RF) distance between the two is simply the sum of unique nodes $(15+15=30)$. The tanglegram was computed using Dendroscope (Huson and Scornavacca, 2012). In this case, the incongruence length difference (ILD) test for partition homogeneity returned a highly significant result $(\mathrm{p}=0.004)$ whereas all tested variants of our incongruence relationship difference (IRD) test were not significant ( $\mathrm{p} \geq 0.11$ ). See text for further explanation. 
Much like the ILD test, this is a randomisation-based test (Fig. 2.3). However, partitions are compared via the distances between the optimal trees that result from them, rather than via tree length (ILD) or a matrix-representation of topology (TILD). There are many possible tree-to-tree distance measures including symmetric difference (RF; Bourque, 1978; Robinson \& Foulds, 1981) quartets distance (QD; Estabrook et al., 1985), nearest neighbour interchange distance (NNID; Waterman \& Smith, 1978), nodal distance (Bluis \& Shin, 2003), maximum agreement subtree distance (Goddard et al., 1994; de Vienne et al., 2007), transposition distance (Rossello \& Valiente, 2006) subtree prune and regraft distance (SPR; Goloboff, 2008), and path-length difference (PLD; Zaretskii, 1965; Williams $\&$ Clifford, 1971). For reasons of familiarity (they are among the most well characterised; e.g. Steel \& Penny, 1993) and ease of use (they are already implemented in $P A U P^{\star}$ ) we chose to use both the symmetric difference (RF) (Fig. 2.4) and the agreement subtree metric (AgD1) as our measures of tree-to-tree distance. We note that all other implementations are possible

Briefly, a heuristic search on each partition was specified using tree bisectionreconnection (TBR), holding up to 500 trees at each cycle, and limited to holding a maximum of 10,000 trees overall. All MPTs from the analysis of each partition were saved and then compared to each other in two different ways. (1) 'Nearest neighbours' (IRD $D_{\mathrm{NND}}$ ) for up to 1,000 trees in each partition: the mean of the minimum distance between each tree in one set, compared with the trees in the other (and vice versa) (Cobbett et al., 2007). (2) The distance between the $50 \%$ majority-rule consensus trees (from up to 10,000 fundamentals) for each partition (IRD $\mathrm{IR}_{\mathrm{MR}}$ ) (Fig. 2.5). We then generated random partitions of the original data in the original proportions, and repeated the above exercises in order to yield a distribution of randomized partition tree-to-tree distances. Distances for the original partitions were deemed significantly different from this distribution when they lay in its $5 \%$ tail. We implemented nearest neighbour and majority rule variants using symmetrical difference distances (IRD $D_{\mathrm{NND}+R F}$ and IRD $D_{\mathrm{MR}+R F}$ respectively) and the majority rule variant with maximum agreement subtree distance $\left(I R D_{M R+A g D 1}\right)$; three statistics in total. Most of our p-values were derived from just 99 replicates (in contrast to the 999 used for ILD and TILD) because of time and computational constraints. 


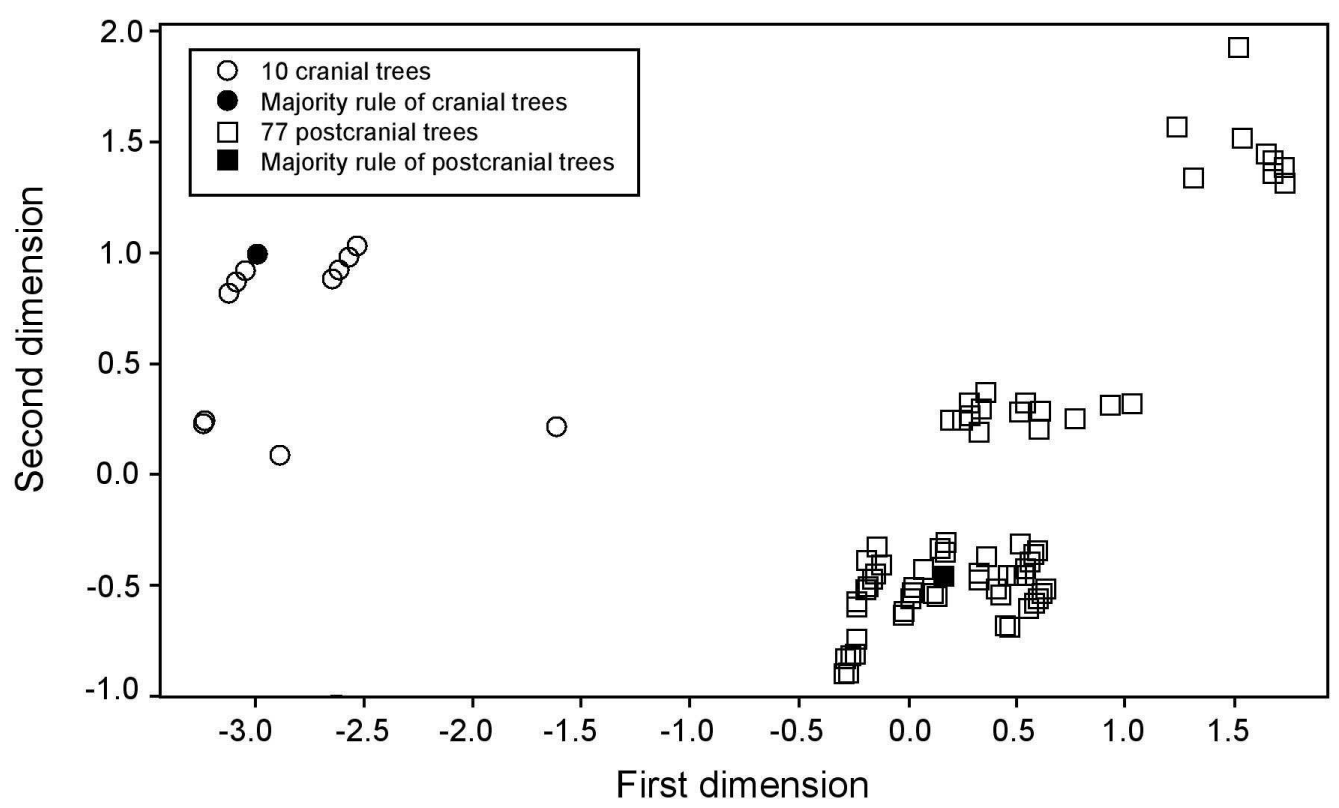

b Maximum Agreement Subtree Distances

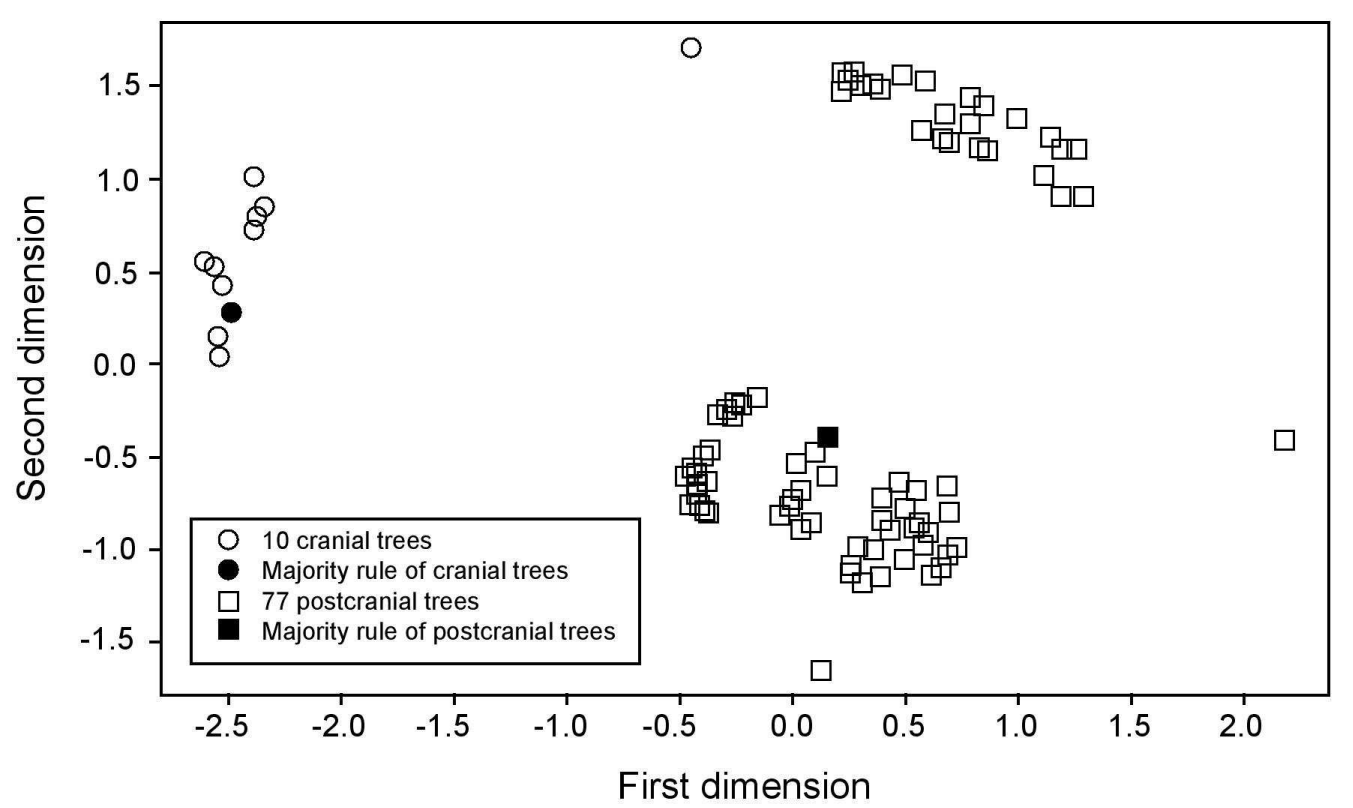

Figure 2.5 Tree-to-tree distances for cranial and postcranial partitions of the mammalian data of Pujos (2007). Distance matrices have been plotted in two dimensions using non-metric multidimensional scaling (NMDS), and rotated using principal components analysis (PCA). Circles indicate cranial trees and squares indicate postcranial trees. Open symbols denote original MPTs, filled symbols (black) denote majority rule trees. a) Robinson Foulds (RF) distances. b) Maximum agreement subtree distances (AgD1). 
Tests not performed in this study. - Rodrigo et al. (1993) proposed three interrelated tests to investigate differences in relationships directly. The first of these determines whether the symmetrical difference distance (RF: Robinson \& Foulds, 1981) between sets of MPTs from independent analyses of the two data set partitions is distinguishable from the distribution of RF distances between a large sample of pairs of random trees. At face value, this is an extremely easy test to pass. It requires only weak congruence between the two data set partitions, and does not assess directly whether the partitions behave as though they are randomly sampled from the same universe of characters. Rodrigo et al's second test does compare the partitions directly (rather than with reference to random trees), and determines if there is any overlap between the MPTs derived from the two partitions upon perturbation of those partitions. Specifically, both partitions are subject to a bootstrapping analysis, and the MPTs obtained are compared. The null hypothesis is that the two data partitions result from the same phylogenetic history, and that there should therefore be "significant" overlap between the two sets (although this is not defined). The protocol is unclear, but suggests that if there are no trees common to both sets then the null can be rejected. Conversely, even one tree common to both sets leads to acceptance of the null. As noted by Lutzoni (1997), this is problematic because the probability of encountering common trees changes with the bootstrap parameters, especially the number of replicates (Page, 1996). The third test is allied to the second, but is more robust. If both partitions of the data set are sampled randomly from the same universe of characters (the null expectation), then the symmetric difference distances between pairs of MPTs from the two partitions should be similar to the distances between pairs of trees obtained by bootstrapping individual partitions. Although a useful test, it may have limitations, particularly where the partitions of the data set are of very different sizes, and especially where the number of characters in the smaller partition is also small relative to the number of terminals. In such cases, bootstraps of the smaller partition may consistently yield poor resolution and low RF distances between trees within this partition (Page, 1996). The IRD test proposed above controls this partition size difference. 


\subsection{Results}

Table 2.1 Meta-summary table classified into significant and non-significant results, for comparisons of cladistic data matrices partitioned into cranial and postcranial. See Appendix 2.1 and online materials for fuller supplementary data.

\begin{tabular}{|c|c|c|}
\hline & $\begin{array}{l}\text { No. Datasets Significantly Different } \\
\text { Between Partitions }(<0.05)\end{array}$ & Exemplars \\
\hline ILD & $18(29 \%)$ & $\begin{array}{c}\text { Choristodera (Matsumoto et al, } \\
\text { 2009) ILD }=0.002\end{array}$ \\
\hline TILD & $50(81 \%)$ & $\begin{array}{l}\text { Amniota }(\text { Hill, 2005) } \\
\text { TILD }=0.001\end{array}$ \\
\hline $\operatorname{IRD}_{\mathrm{NND}+\mathrm{RF}}$ & $20(32 \%)$ & $\begin{array}{l}\text { Ankylosauria (Osi et al, 2009) } \\
\text { IRD }_{\mathrm{NND}+\mathrm{RF}}=0.010\end{array}$ \\
\hline \multirow[t]{2}{*}{$\mathrm{IRD}_{\mathrm{MR}+\mathrm{RF}}$} & $18(29 \%)$ & $\begin{array}{c}\text { Plateosauria (Smith \& Pol, 2007) } \\
\operatorname{IRD}_{\mathrm{MR}+\mathrm{RF}}=0.020\end{array}$ \\
\hline & $\begin{array}{l}\text { Significance of difference across all } 62 \\
\text { datasets, between partitions }\end{array}$ & Inference \\
\hline CI & $\begin{array}{c}\text { Significant } \\
\text { (paired } \mathrm{t}=-2.2278, \mathrm{p}=0.0296)\end{array}$ & $\begin{array}{l}\text { Postcranial significantly higher } \\
\text { CI than cranial }\end{array}$ \\
\hline RI & $\begin{array}{c}\text { Not Significant } \\
\text { (paired } \mathrm{t}=1.0489, \mathrm{p}=0.2984 \text { ) }\end{array}$ & $\begin{array}{c}\text { Cranial higher RI on average, but } \\
\text { not significant }\end{array}$ \\
\hline Mean ci & $\begin{array}{c}\text { Not Significant } \\
(\text { paired } t=-1.3422, p=0.185)\end{array}$ & $\begin{array}{l}\text { Postcranial per character ci } \\
\text { higher but not significant }\end{array}$ \\
\hline Mean ri & $\begin{array}{c}\text { Significant } \\
\text { (paired } \mathrm{t}=4.7324, \mathrm{p}=1.36 \mathrm{e}-05 \text { ) }\end{array}$ & $\begin{array}{l}\text { Cranial per character ri } \\
\text { significantly higher }\end{array}$ \\
\hline HER & $\begin{array}{c}\text { Significant } \\
\text { (paired } \mathrm{t}=2.042, \mathrm{p}=0.0455)\end{array}$ & $\begin{array}{c}\text { Cranial HER significantly higher } \\
\text { than postcranial HER }\end{array}$ \\
\hline
\end{tabular}




\subsubsection{Homoplasy and Resolution in Cranial and Postcranial Data Partitions}

Across our sample of 62 data sets, cranial partitions had significantly more characters $($ median $=66)$ than postcranial partitions $($ median $=54.5)($ Wilcoxon signed ranks; $V=$ 1398, $p=0.0032$ ) (Appendix 2.1). This difference would have been more marked were it not for the inclusion of multiple ornithological studies (e.g., Bourdon et al., 2009; Ksepka, 2009; Worthy, 2009) that are counter biased towards greater numbers of postcranial characters. We also selected matrices containing relatively balanced numbers of characters a priori, making a significant difference less likely.

Differences in numbers of characters across partitions mean that it is not straightforward to compare ensemble consistency indices $(\mathrm{Cl})$ and retention indices $(\mathrm{RI})$. Nonetheless, we observe that the mean Cls for cranial and postcranial characters across all 62 data sets were significantly different (paired $t=-2.2278, p=0.0296$ ), with postcranial partitions $(\bar{x}=0.549)$ having slightly higher self-consistency than cranial partitions $(\bar{x}=$ 0.512) overall. This difference may, in part, be a function of the smaller average number of characters within postcranial partitions. Indeed, using the mean partition (per character) ci index across all matrices revealed a difference in the same direction $(\bar{x}=0.560$ and 0.540 for postcranial and cranial partitions respectively), but this was not significant (paired $t=-1.3422, p=0.185)$. Mann-Whitney tests of cranial and postcranial ci values within our 62 data sets yielded fourteen significant $(p<0.05)$ results (three or four might be expected). Ten of these fourteen had higher means (less homoplasy) for postcranial partitions; an insignificant bias (binomial test, $p=0.180$ ) that was nonetheless in general agreement with the other ways of expressing differences in $\mathrm{Cl}$ and ci.

No significant difference was found in the test of cranial versus postcranial ensemble retention indices $(R I)$ across data sets $(t=1.0489, p$-value $=0.2984)$ but per character retention indices $(r i)$ showed a highly significant difference $(t=4.7324, p=$ 1.36e-05) in the opposite direction (higher values in cranial partitions) from the $\mathrm{Cl}$ and ci. Higher Homoplasy Excess Ratio (HER) values were also found in the cranium; the mean value for cranial partitions $(0.5675)$ was significantly higher than that for postcranial partitions $(0.5207)(t=2.042, p=0.0455)$. Our results are equivocal, therefore, depending upon precisely how homoplasy is measured.

The strict consensus fork index (CFI; Colless 1980; Table 2.1) also demonstrated that the number of nodes resolved in cranial partitions is greater than that in postcranial 
partitions. Only 16 cranial partitions resulted in a strict consensus with no resolution $(\mathrm{CFI}=$ 0 ), compared with 25 postcranial partitions. All partitions for all data sets had a CFI greater than zero for their majority-rule (>50\%) consensuses. A comparison of majority-rule CFIs between cranial and postcranial partitions reveals that the former give significantly better resolution (paired Wilcoxon; $V=1190.5, p=0.004$ ).

\subsubsection{Congruence between Cranial and Postcranial Signals (ILD tests)}

When originally described, the ILD test was used with a standard significance level of $5 \%$ (0.05). At this level, 19 of our 62 data sets had significant character incongruence between cranial and postcranial partitions (Table 2.1). Whilst this significance level remains the most widely quoted, some have suggested more stringent significance levels should be used (e.g., Cunningham, 1997a). Applying stricter significance levels, we reject the null for just $16(p<0.010)$ and $8(p<0.001)$ of our data sets.

The Similarity of Relationships Implied by Cranial and Postcranial Partitions

TILD and IRD tests. -50 of our data sets (>80\%) had partitions for which majority-rule trees yielded significant TILD tests results ( $p \leq 0.05$ ); 39 of these also had $p=0.001$. We used 100 replications for our IRD tests because of time constraints. Using the nearest neighbour procedure with symmetrical difference distances (IRD $\mathrm{NND+RF}), 20$ of our data sets $(32 \%)$ had significantly incongruent relationships implied by cranial and postcranial partitions $(p \leq 0.05)$ (Table 1). Using majority rule trees and symmetrical difference distances $\left(\mathrm{IRD}_{\mathrm{MR}+\mathrm{RF}}\right)$ yielded highly similar but not identical results $\left(r=0.80, p=6.21 \times 10^{-}\right.$ $\left.{ }^{15}\right)$; 18 data sets (29\%) had significantly incongruent relationships. There are two reasons for the differences. Firstly, majority rule trees embody most frequent relationships rather than 'average' relationships (Fig. 3), so the two tests address different questions. Secondly, the IRD $D_{\mathrm{NND}+\mathrm{SR}}$ was calculated using up to 1,000 trees from each partition (a maximum of 499,000 tree-to-tree distances for each replicate), but these may not offer representative samples of all islands of MPTs where the latter are very numerous.

The analogous tests using maximum agreement subtree distances (AgD1) were only implemented for majority rule trees, since these distance computations were very much slower in $P A U P^{*}$, encountered segmentation faults (in beta 10) and became 
prohibitively time consuming for thousands of neighbour comparisons. The IRD $\mathrm{MR}_{\mathrm{AgD} 1}$ test revealed significantly different $(p \leq 0.05)$ majority rule trees for seventeen data sets. Correlation with $\mathrm{p}$-values from the IRD $\mathrm{D}_{\mathrm{MR}+\mathrm{RF}}$ test were significant but not especially high (Kendall's $\pi=0.1895, p=0.0350$ ) because the two metrics address different aspects of tree-to-tree distance.

Partitioning data sets into four broad taxonomic groups (Mammalia, Avemetatarsales/Ornithodira, fishes and 'other tetrapods') revealed some striking differences, albeit with modest sample sizes. In particular, fishes were more likely to have congruent cranial and postcranial partitions than the tetrapod groups.

Templeton, winning sites and KH tests. - These tests are not straightforward to interpret, because rather than comparing two alternative trees with an independent data set, we were here comparing the optimal tree from a given data set with an alternative (suboptimal by definition). This will yield a high rate of type I errors (Planet, 2006). Moreover, these comparisons were necessarily mediated via consensus trees, which may not be included in the set of fundamentals and may therefore also be suboptimal. Unsurprisingly, therefore, the majority of the tests reported significant differences (see online Appendix 1). Results using majority rule and strict consensus trees were very similar. We summarize these here as the tally of data sets for which optimization of both partitions and the entire data set onto the cranial and postcranial trees yielded significant p-values (i.e., where all three comparisons yielded $\mathrm{p} \leq 0.05$ ). For both Templeton and $\mathrm{KH}$ tests (both using the magnitude of step differences per character), 38 data sets (61\%) had a maximum $p \leq 0.05$ using majority rule trees, while $41(66 \%)$ had a maximum $p \leq 0.05$ using strict consensus trees. The winning sites test (utilizing only the direction of the step differences) was a little more conservative, reporting a maximum $p \leq 0.05$ for 31 data sets using the majority rule and 38 using strict consensus trees.

As above, these statistics consistently reported more congruent cranial and postcranial partitions for fishes than for the terrestrial groups. However, these differences were not significant with the possible exception of the winning sites test with majority rule trees, which was marginal ( $G=6.8299$, Lo-squared $d f=3$, p-value $=0.0775)$. 


\subsection{Discussion}

\section{Cranial and Postcranial Partitions Contain Similar Levels of Homoplasy}

We are cautious when interpreting consistency indices in data set partitions, because these partitions seldom comprise identical numbers of characters. Moreover, there is a significant bias toward higher numbers of cranial characters across our sample of data sets. On one hand, the ensemble $\mathrm{Cl}$ for a partition optimized parsimoniously in isolation is 'biased' by the number of characters (Fig. 2.2); larger partitions can be shown to have a lower Cl in random simulations (cf. Sanchez-Villagra \& Williams, 1998; Song \& Bucheli, 2010). We did, indeed, find a significant difference in ensemble $\mathrm{Cl}$ between our partitions (paired $t=-2.2278, p=0.0296$ ), with postcranial partitions having higher values overall. On the other hand, if both partitions are analysed simultaneously, then mean or median per character ci values are likely to be higher in the larger partition; at least in the hypothetical case where the partitions contain conflicting signals of similar strength per character (Fig. 1). We did not find a bias in this direction; indeed, there was no significant difference between the cranial and postcranial per character ci values.

We produced a simple linear model expressing partition $\mathrm{Cl}$ in terms of the log of the number of taxa and the log of the number of characters across all 124 partitions. All terms were highly significant (multiple $R^{2}=0.4965, p=2.2 e-16$ ). A subsequent paired $t$-test of the residual $\mathrm{Cl}$ values from this model revealed no significant difference $(t=-0.5535, p=$ 0.5820 ) between cranial and postcranial partitions. We note that other variables have been demonstrated empirically to influence $\mathrm{Cl}$ (Donoghue \& Ree, 2000; Hoyal Cuthill et al., 2010), but our simple model was sufficient to remove the apparent discrepancy between cranial and postcranial $\mathrm{Cl}$ values in this case.

There was a highly significant difference between cranial and postcranial partition RI values (Wilcoxon test: $V=1613, p=1.660 \mathrm{e}-06$ ); more homoplasy in the latter, and the opposite pattern to partition $\mathrm{Cl}$ and mean per character ci. The $\mathrm{RI}$ was only marginally influenced by the log of the number of characters in a simple linear model (multiple $\mathrm{R}^{2}=$ $0.0290, p=0.1682$ ), and the residual RIs from this model were highly significantly different too $(V=1615, p=7.71 e-06)$. Homoplasy Excess Ratio $(H E R)$ values were also significantly different $(t=2.042, p=0.0455)$, with cranial partitions having a higher mean $(0.5675)$ than postcranial partitions $(0.5207)$; more homoplasy in the latter. However, when the (admittedly non-significant) effects of matrix dimensions were modelled out (multiple $\mathrm{R}^{2}$ 
$=0.0042, p=0.7758)$, there was no significant difference in residual HER $(t=1.8519, p=$ 0.0689 ). Levels of homoplasy in cranial and postcranial partitions appear to be broadly similar, therefore, with differences detected in opposite directions for the partition $\mathrm{Cl}$ and mean per character ci on one hand, and the RI and HER on the other hand (prior to controlling for matrix dimensions).

We strongly advocate the use of the HER rather than the ensemble $\mathrm{Cl}$ as an index of homoplasy and data quality. However, we note a possible complication in the calculation of the HER with respect to the distribution of missing values in a matrix. In particular, the procedure does not distinguish between known states (the data) and missing entries (which are not data). The effects of missing entries are strongly dependent upon their distribution. A taxon for which all characters code '?' can resolve anywhere in the network with no cost. This will obfuscate the search for MPTs and will obliterate any resolution in the strict consensus. When these same missing entries are randomly redistributed across all taxa, it becomes highly unlikely that individual terminals will bear such a high concentration of '?'s, and resolution becomes more probable. As such, the precise distribution of missing entries may more properly be regarded as an intrinsic property of the matrix (one that should be held constant, along with the frequency distribution of states across characters) rather than as data to be permuted along with the known states. An allied problem is the treatment of additive binary codings and contingent characters. In the former case, the permutation of codes in successive linked columns may yield meaningless combinations, but this is easily overcome with the use of multistate ordered coding. In the latter case, however, the position of an inapplicable code is contingent upon the state of some other character. For example, one character codes for the presence (1) or absence $(0)$ of some feature and the second codes for the form of that feature (coded '?' if the first character codes ' 0 '): permutation again yields meaningless combinations. Although this can be circumvented with the use of more inclusive multistate characters, these do not convey the same information and make different assumptions regarding homology. Blocks of contingent characters are more appropriately regarded as specifying particular types of character state trees. We therefore suggest that the HER permutation step might be modified in two ways; firstly by keeping the positions of missing entries static and secondly by permuting the codes for contingent characters as blocks (c.f. Wilkinson, 2001). These undesirable missing data effects account for occasional estimates of the HER below zero (e.g., Gonzalez-Riga et al. 2009; postcranial partition).

We note that the absence of a clear difference between cranial and postcranial 
levels of homoplasy does not necessarily imply that additional characters of equivalent phylogenetic informativeness can be garnered from the two partitions with comparable ease. For example, a dataset may contain 100 cranial and 100 postcranial characters with identical levels of homoplasy. However, the postcranial characters may have been selected from amongst many (potentially more homoplastic) candidates with enormous care (and represent all the practically extractable data), whereas all potential cranial characters could be of uniformly high quality and of much greater abundance. Our conclusions therefore necessarily relate to the coded data.

\subsubsection{A Significant Minority of Cranial and Postcranial Partitions have Incongruent Signals}

Our ILD test results demonstrate that the majority of our 62 sampled data sets (69\%) have congruent cranial and postcranial character partitions. However, this leaves 19 data sets in which there is significant incongruence (Allain \& Aquesbi, 2008; Anderson et al., 2008; Asher et al., 2005; Asher, 2007; Beck et al., 2008; Ezcurra \& Cuny 2007; Friedman et al., 2007; Gaubert et al., 2005; Hill, 2005; Holland \& Long, 2009; Li et al., 2007; Matsumoto et al., 2009; Ruta \& Coates, 2007; Sanchez-Villagra et al., 2006; Spaulding et al., 2009; Sues \& Reisz, 2008; Vallin \& Laurin, 2004; Wiens et al., 2005; Worthy, 2009). Assuming a significance level (false positive rate) of $5 \%$, one would expect three or four data sets to be significantly incongruent by chance alone $(0.05$ x $62=3.1)$. Our results therefore suggest that significant incongruence is being detected across our sample of data sets (binomial test $p<0.00001$, assuming a $5 \%$ false positive error rate). We must stress that we make no inferences concerning the overall quality of individual data sets on the strength of these results. In each case, the original authors analysed all cranial and postcranial characters together; we imposed the partitions. One conclusion of this paper is that characters should be sampled from all aspects of morphology rather than focusing upon one region. This was the broad approach taken in the original publications (indeed, we selected examples where there was a reasonable balance of cranial and postcranial characters).

It is reasonable to ask whether incongruence is a global phenomenon, or whether it is concentrated in particular taxa (Rodrigo et al. 1993). Hence, we explored the effects of removing single taxa in a series of first order taxon jack-knifing ILD tests (see online Appendix 3). These highlighted several terminals with marked effects. For example, the 
global incongruence in Ezcurra \& Cuny (2007) was attributable to just one taxon, Dilophosaurus wetherilli. Removing this species resulted in a p-value of 0.236 (rather than 0.005 ), whereas removing any of the other 13 terminals still yielded $p<0.05$. Similarly, the significant ILD result for Allain \& Aquesbi $(2008)(p=0.005)$ was entirely contingent upon the inclusion of Rapetosaurus. Its removal yielded $p=0.168$, whereas the exclusion of any of the other 23 taxa resulted in a maximum p-value of 0.015. In Sues \& Reisz (2008), incongruence was caused largely by the inclusion of Scutosaurus ( $p$ increased to 0.719 when it was deleted). In this particular data set, the deletion of three other taxa (Lanthanosuchoidea, Owenetta kitchingorum and Sclerosaurus) also resulted in $p<0.05$, although the effect was more marginal. Incongruence in the data of Holland \& Long (2009) $(p=0.008)$ largely disappeared with the exclusion of Eusthenopteron $(p=0.578)$ and was significantly reduced with the exclusion of Gogonasus and Tiktaalik $(p=0.118$ and 0.124 respectively). Finally, the significant ILD incongruence detected in the data set from Sanchez-Villagra et al. (2006) was variously contingent upon taxon-sampling (median $p=$ 0.057 , with a minimum of 0.010 and maximum of 0.303 for 20 single taxon deletion ILD tests). In contrast to these examples, the data sets of Anderson et al. (2008), Beck et al. (2008), Li et al. (2007) and Gaubert et al. (2005) remained significantly incongruent no matter which single taxa were deleted (the last of these retained $p=0.001$ throughout). Time constraints prevented us from applying first order taxon jack-knifing ILD tests to all of the significantly ILD-incongruent data sets in this way.

We also applied the first order taxon jack-knifing ILD to data sets that passed the original partition homogeneity test (i.e., $p>0.05$ ). For example, in the borderline case of the data from Pujos et al. (2007) (ILD p-value = 0.072), 17 single taxon jack-knifed variants yielded a median $p$-value of 0.072 , with a minimum of 0.005 and maximum of 0.229 . Again, although the precise taxon sample influenced the ILD result, the median and mean ILD p-values of first-order taxon jack-knifed variants were similar to those for the entire (all taxon) dataset. This was true in all of the 38 data sets we tested in this manner, suggesting that the ILD test generally offers a robust assessment of congruence despite the marked impact of particular taxa. 


\subsubsection{Cranial and Postcranial Partitions Imply Significantly Different Relationships in a Significant Minority of Cases}

The vast majority of our topological incongruence length difference (TILD) tests reported significant differences for majority rule $(>80 \%)$ trees of cranial and postcranial partitions. The TILD test, at least as interpreted here, appears very difficult to pass, and we suspect a high rate of Type I errors. Even data sets with comfortably high ILD test p-values (i.e., Beard et al., 2009; Gonzalez-Riga et al., 2009) yielded significant p-values from the TILD.

Results from the variants of our incongruence relationship difference (IRD) test were much more similar (although not identical) to those from the ILD test. For the symmetrical difference distance (Robinson \& Foulds, 1981) based upon nearest neighbours (IRD $\mathrm{NND}_{\mathrm{NR}}$ ) and the majority rule consensus (IRD $\left.\mathrm{MR}_{\mathrm{MR}}\right), 37 \%$ and $36 \%$ of data sets respectively yielded significantly different trees from the two partitions. For the test based upon the maximum agreement subtree distances and majority rule trees $\left(\operatorname{IRD}_{\mathrm{MR}+\mathrm{AgD1} 1}\right), 21 \%$ of data sets yielded significant differences (the nearest neighbour variant, IRD $D_{N N D+A g D 1}$, was not implemented). As with the ILD test, we would expect three or four data sets $(5 \%)$ to reject our null of homogeneity by chance. Our results using this alternative test of partition homogeneity therefore indicate that a significant minority of cranial and postcranial partitions imply different phylogenies. Of the 19 dataset partitions determined to contain incongruent signals by the ILD test, 12 also implied incongruent relationships $(p<0.05)$ using the IRD ${ }_{\mathrm{NND}+\mathrm{SR}}$ test, 10 using the IRDMR+SR test and 4 using the IRD $\mathrm{IR}_{\mathrm{Mgg} 1}$ test. Previous studies have suggested that the Robinson Foulds (RF) distance measure may be poor at discriminating between congruent and incongruent partitions. This is because the distribution of RF-distances between randomly generated trees (or between trees generated by a time-homogeneous birth-death model) is highly skewed and has a comparatively narrow range of sensitivity (Koperwas \& Walczak, 2011; Lin et al., 2011). However our empirical results indicate that the (IRD $\left.D_{N N D+S R}\right)$ test application of this measure not only generates a full-spectrum of values (between 0.910 and 0.01 ), but also tends to agree with the ILD test assessments of incongruence. We note that faster parsimony programs and more efficient algorithms for calculating tree-to-tree distance metrics (e.g., Goloboff et al., 2008; Pattengale et al., 2007) would increase the computational speed of these tests and allow nearest neighbour distances to be calculated between greater numbers of MPTs. Our cap at 1,000 trees in each partition mean that IRD $D_{N N D+S R}$ results must be treated with caution (although $I R D_{M R+S R}$ and $I R D_{M R+A g D 1}$ results were usually based 
upon consensus representations of all MPTs). We also note that there are many other possible tree-to-tree distance metrics that could be implemented in such tests, several of which may have more desirable properties than RF and AgD1 (Lin et al., 2011).

\subsubsection{What do these Results Imply for Cladistic Analyses of Morphology?}

In studying the evolution of form, it is now relatively common to recognize anatomical modules. These are regions of the body (or suites of landmarks) within which morphological changes are strongly correlated through evolutionary time, but between which there is significantly less coordination. Different selective forces may operate on these modules, and they may therefore exhibit different evolutionary trends (Mitteroecker \& Bookstein, 2007; Klingenberg, 2008). In the context of phylogenetic characters, differing pressures on modules may favour particular patterns of convergence and homoplasy, and therefore suites of characters that imply different trees. The skull of many tetrapod groups has often been regarded as biomechanically and functionally somewhat independent of the rest of the skeleton (Ji et al., 1999; Koski, 2007; Mitteroecker \& Bookstein, 2008) hence the difficulty of making many inferences about the one from the other.

Does it matter, therefore, which morphological characters we choose to code when attempting to infer phylogeny? It is difficult to escape the conclusion that it does: whether because the a priori omission of characters believed to be analogous or strongly homoplastic is standard practice (although rarely documented explicitly), because alternative data sets for identical sets of taxa often yield radically different trees, because the tree(s) derived from a given data set can alter markedly with the omission or reweighting of characters (most obviously when bootstrapping), or because characters are subject to different selective pressures in different modules. The usual approach in morphological phylogenetics is to combine all available data (Kluge et al., 1989). Although it is acknowledged that the patterns inferred from particular organ systems or suites of characters may be misleading (in the same way and for the same reasons that individual characters may merely introduce homoplasy and noise), it is hoped that the combined analysis of all available characters will allow the true phylogenetic signal to emerge from conflicting local homoplasy. How many characters are enough for the emergence of a reliable signal? The issue, naturally, is one of scale, and concerns the quality of signal 
typically encountered in character matrices of the dimensions actually generated in real vertebrate case studies, as well as how these characters are distributed across putative modules. What we can say, however, is that in studies of this type, an exclusive focus upon characters of either the cranium or postcranium (at the expense of those of the other partition: e.g., Fitzgerald (2010) (craniodental only) and Mayr \& Mourer-Chauvire (2004) (postcranial only)) will significantly influence the resultant optimal tree(s) about one time out of three. This is above the baseline expected for merely sampling a smaller number of characters. We suggest that such a practice is inadvisable, therefore, and strongly advocate garnering character data from all anatomical regions. Certainly, we find little evidence to suggest that cranial or postcranial characters contain different levels of homoplasy (once partition sizes are controlled). Hence, our sample of 62 case studies offers little justification for concentrating on, for example, characters of the skull, because these are believed a priori to be of greater value in attempting to infer phylogeny. However, where cranial and postcranial signals conflict, contain similar levels of noise and are of unequal size, then the mean per character consistency (ci) is likely to be lower for the smaller partition (Fig. 1). Such approaches to measuring character quality may give the misleading impression of a 'cleaner' phylogenetic signal in the more highly sampled partition. Because systematics (like all science) builds upon prior knowledge, this may account for the practice whereby successive studies of certain groups allocate increasingly intensive sampling efforts to particular anatomical regions (as explained by Arratia 2009, and also noted in Joyce \& Sterli 2012): the process potentially becomes cyclical and selfreinforcing.

When coding fossils, we may not be able to sample across the same suite of characters that we would employ with extant species (Wiens, 2003a,b; Cobbett et al., 2007). For example, in fossil crocodyliformes, the vast majority of characters necessarily come from the cranium (e.g., Hastings et al., 2011; Puertolas et al., 2011; Cau \& Fanti, 2011; Turner \& Sertich, 2010; O'Connor et al., 2010) and it is difficult to be confident that we are not merely inferring a 'cranial' tree. The only (and indirect) way to answer this would be to conduct parallel tests upon the closest living representatives of the clade. However, the (quite possibly limited) utility of this approach depends upon the phylogenetic proximity of the extant exemplars, the presumed constancy of selective pressures on putative modules through time and across clades (a big assumption: Hunt, 2008; Frazzetta 2012), and the similarity of the available coded data. Hence, while this may be a sensible approach for crocodiles, it offers little in groups whose closest extant 
relatives are ecologically or morphologically very divergent from the fossils (e.g., non-avian dinosaurs, osteostracans), or which are entirely extinct.

A related issue in the context of fossil vertebrates is the preferential preservation of hard part characters (bones rather than muscles or other more volatile tissues). An analogous concern, therefore, is whether skeletal and soft-part characters convey an homogeneous phylogenetic signal (Diogo, 2004, p405-416). If not, this has implications for the manner in which fossil vertebrates are interpreted and analyzed (Sansom et al., 2010) and is an area in particular need of detailed future work.

\subsection{Conclusions}

- Systematists typically abstract significantly more characters from the cranium than the postcranium. Tests for levels of homoplasy in the cranial and postcranial partitions of our data sets were equivocal, depending upon how homoplasy was assessed. Although postcranial partitions had a significantly higher mean ensemble consistency index $(\mathrm{Cl})$ than cranial partitions, this difference disappeared when dataset size parameters were modelled out. When all data were analysed simultaneously, mean per character consistency indices (ci) were also higher for postcranial than cranial partitions (although not significantly so). By contrast, the HER (which ameliorates the biasing affects of partition size imbalance using a randomisation test) reported the opposite trend: significantly higher levels of homoplasy in postcranial than cranial partitions.

- Whilst combined analyses using all the evidence available may be optimal for phylogenetic reconstruction, the relative congruence of constituent morphological data partitions has rarely been explored. We are the first to do this with a moderately large sample size (62 data sets), applying a consistent methodology and approach. Cranial and postcranial anatomy implied significantly different phylogenies in about a third of our data-sets. Focus on either source of character data to the exclusion of the other may therefore be inadvisable. We tentatively attribute observed incongruence to the operation of disparate selective pressures acting upon different regions of the body. These yield patterns of homoplasy that may mislead parsimony analyses in one or both cases. More generally, we argue that signals within subsets of morphological data should be examined more 
routinely.

- Many different metrics of partition homogeneity and congruence are possible, and we have explored only a subset of them here. While the results from the ILD and the implemented variants of our new IRD test were in broad agreement, there were many detailed differences. These differences may help to illuminate the nature of incongruence by exploring several properties of a matrix (Planet, 2006). Similarly, first order taxon jack-knifing offers a means to localise any incongruence that is detected.

- As measured by variants of our new IRD test, trees derived from cranial and postcranial characters of fishes were more similar to one another than were trees derived from partitions of other vertebrate groups. We tentatively suggest that this may reflect some greater degree of modularity in the cranium of tetrapods relative to fishes. There were no significant differences in ILD results between groups. 


\section{Chapter 3: An Updated Examination of the Impact of the Fossil Taxa in Parsimony Analyses of Morphology}

\subsection{Abstract}

In a previously published comparative cladistic analysis (Cobbett et al.. 2007), forty-five different cladistic data sets were re-analysed to compare the relative contribution of fossil and extant taxa to the resolution, topology, leaf stability and homoplasy as inferred from parsimony analyses. In this chapter I shall extend this work by using more data sets, newer data sets, data sets containing more characters, more taxa and data sets representing new previously unsampled groups such as plants, echinoids, wasps, chitons, and sea spiders. A quicker, more computationally-efficient pipeline to perform these analyses is introduced; using new technology searches in TNT, tree-to-tree distance comparisons in R, and leaf stability analyses in RogueNaRok (Aberer et al. 2013). Comparisons of topological distance based upon mean path difference, in addition to the mean Robinson-Foulds distance are used. On average there are barely any significant differences across all data sets found between fossil and extant taxa in these analyses. Within this, there are however some notable datasets, and notable taxa which we discuss further. Overall though, this further confirms and strengthens the findings reported by Cobbett et al. (2007) that fossil taxa are little different in their effect, on average in cladistic analyses of morphology. 


\subsection{Introduction}

Traditionally, palaeontological and neontological approaches to phylogenetic inference were rather separate. It is only in the last 30 years or so that both fossils and extant taxa have regularly been analysed together in cladistic analyses. Fossils offer a unique snapshot view of past evolutionary morphologies along with approximate temporal and environmental information (Adrain et al. 2001). They may help break up long branches and provide sources information that our closer to splitting events (Chapter 1), and they allow provide an independent "reality check" on molecular phylogenetic hypothesis (Jenner 2004; Wiens 2004; Smith \& Turner 2004).

But the empirical effect of fossil taxa relative to extant taxa across a broad range of taxa has rarely been tested. One robust effort came from Cobbett et al. (2007) who examined the difference in relationships when single taxa were deleted (first order jackknifing) from mixed analyses of extant and extinct taxa. By comparing 'whole' dataset statistics (MPTs, $\mathrm{Cl}, \mathrm{RI}$, relationships, and leaf stability) to the same statistics from taxon-jackknifed variants, Cobbett et al. (2007) built a fair test in which we can observe, compare and isolate the properties of fossil and Recent taxa. Through their analyses they demonstrated that in some data sets e.g. Dong (2005) the exclusion of key fossil taxa such as Ottoia can have a dramatic affect on the resulting phylogenetic inference - in the case of Ottoia exclusion it resulted in a significant loss of resolution as measured by the consensus fork index (CFI; Colless 1980), and a significant increase in MPTs. Even more remarkable is that Ottoia is scored $38 \%$ missing or inapplicable in Dong's (2005) matrix.

That example was one extreme, but across the entire sample of 45 data sets, fossil taxa and Recent taxa had statistically insignificant differences for most effects tested: homoplasy, number of MPTs, and relationships. Only in the case of leaf stability were there noticeably more data sets in which fossil taxa were significantly less stable than recent taxa. The computational constraints at the time mean't that leaf stability could not be calculated for all the data sets in the sample, so only 36 data sets were assessed for comparative leaf stability.

With the advent of MorphoBank (O'Leary \& Kaufman, 2011), and Graeme Lloyd's (2009) online collection of matrices there is now more cladistic data online to choose from for this 
type of comparative cladistic analysis. Whilst those archived matrices represent perhaps only $4 \%$ of the phylogenetic studies that have been published (Stoltzfus et al. 2012) - it certainly provides a good base to start from in an attempt to extend beyond the analyses of Cobbett et al (2007) to a more diverse range of fossil groups and to sample data that is newer, and larger in both taxa and characters. In this chapter I re-analyze data sets such as Legg et al (2012) with over twice as many taxa as the largest data set looked at Cobbett et al. (2007), to further examine the impact of fossil taxa in mixed analyses.

\subsection{Methods}

\subsubsection{Finding Appropriate Published Data in the Literature}

data sets were sourced either directly from the literature by myself, or sourced from phylogenetic data stores such as MorphoBank (O'Leary \& Kaufman, 2011), TreeBASE (Piel et al., 2009) and Graeme Lloyd's collection of matrices (www.graemetlloyd.com). I also tried the literature search method given in Cobbett et al. (2007) but found it to be of limited value. For example, disappointingly few of the 14 articles in the Systematic Entomology virtual issue on 'Systematics of Fossil Insects' (2009) contained morphologybased cladistic analyses with enough fossil taxa in them $(\mathrm{N}>3)$ to be of use for this study, despite many of them coming-up in the literature search. Limited access to journal articles as ever was also a significant problem in identifying suitable data sets for inclusion, as rather few of the titles and abstracts made it obvious that the published study contained a cladistic analysis of fossil and extant taxa. A lot of papers were sought that looked promising from the title and abstract but upon receipt of the full text (not always always via quick or easy means) were found to be lacking in usefulness for this particular study and its criteria. In this respect I must especially commend MorphoBank in particular for providing additional metadata that particularly aids the identification of mixed extant/extinct taxon data sets: the 'Taxa' page for each matrix e.g. this one corresponding to Spaulding \& Flynn's (2012) analysis of Carnivoramorpha

(http://www.morphobank.org/index.php/Projects/Taxa/project_id/367) can in many cases quickly and clearly identify which taxa are extinct and which taxa are extant, enabling easy discovery of data sets appropriate for re-use in this context. However, it must be noted that not all data uploaders have added this metadata (e.g Beutel et al.'s (2012) analysis of 
Adephaga has only one item of marked-up taxonomic metadata (http://www.morphobank.org/index.php/Projects/Taxa/project id/814)). Still, it is much appreciated that the authors uploaded their data in it's raw, immediately re-usable format to MorphoBank rather than not at all. The matrices I sourced directly from the literature were much more arduous to reformat back into re-usable data and validate. TreeBASE is ill-equipped and (to be fair) not designed to facilitate searching for proportions of palaeontological taxa. A search for "fossil" in the "All Text" field would seem to be the most valuable approach for this database, and this search currently yields over 200 items.

Due to all the above intricacies of appropriate data discoverability, I acknowledge that my sampling of the literature is thus inadvertently biased towards sampling:

1) studies that have deposited their cladistic data in MorphoBank or TreeBASE or are on Graeme Lloyd's site

2) studies published in journals I had legitimate, immediate full-text access to e.g. those in popular journals, Open Access journals, or where the author(s) have kindly made a freely available and discoverable full-text copy of the work on the internet ('green' Open Access).

3.) studies published more recently, from $2009-2013$ that I am naturally more likely to be aware of as I was actively engaged in research during this time.

\subsubsection{A new taxon-jackknifing analytical pipeline}

Having tried using the DELBAT/DELSUM scripts from Cobbet et al. (2007) it became apparent that they were not going to scale well for some of the larger data sets I had collected e.g. Legg et al.'s (2012) analysis of arthropod phylogeny which is a matrix of 173 taxa and 580 characters. Nevertheless I tried using them, and soon found additional problems: PAUP* 4.0b10 in particular has a known, reproducible and unsolved bug whereby the AgD1 and AgD tree-to-tree distance algorithms will cause PAUP* to crash if passed certain trees to compare. I have provided example data and scripts in Appendix 1 to demonstrate this bug with a simple comparison of one valid MPT to one other valid MPT that consistently crashes PAUP*. There is also the considerable slowness of doing traditional searches in PAUP* relative to the New Technology search methods (Goloboff 1999; Nixon 1999) now available in TNT. 
Thus after I had gone to the TNT workshop in California and received expert one-to-one tutelage in scripting TNT it seemed like I should attempt to create a brand-new modernised analysis pipeline to implement some of the taxon-deletion tests of Cobbett et al. (2007). A sketch of the new analytical pipeline is provided in Figure 1

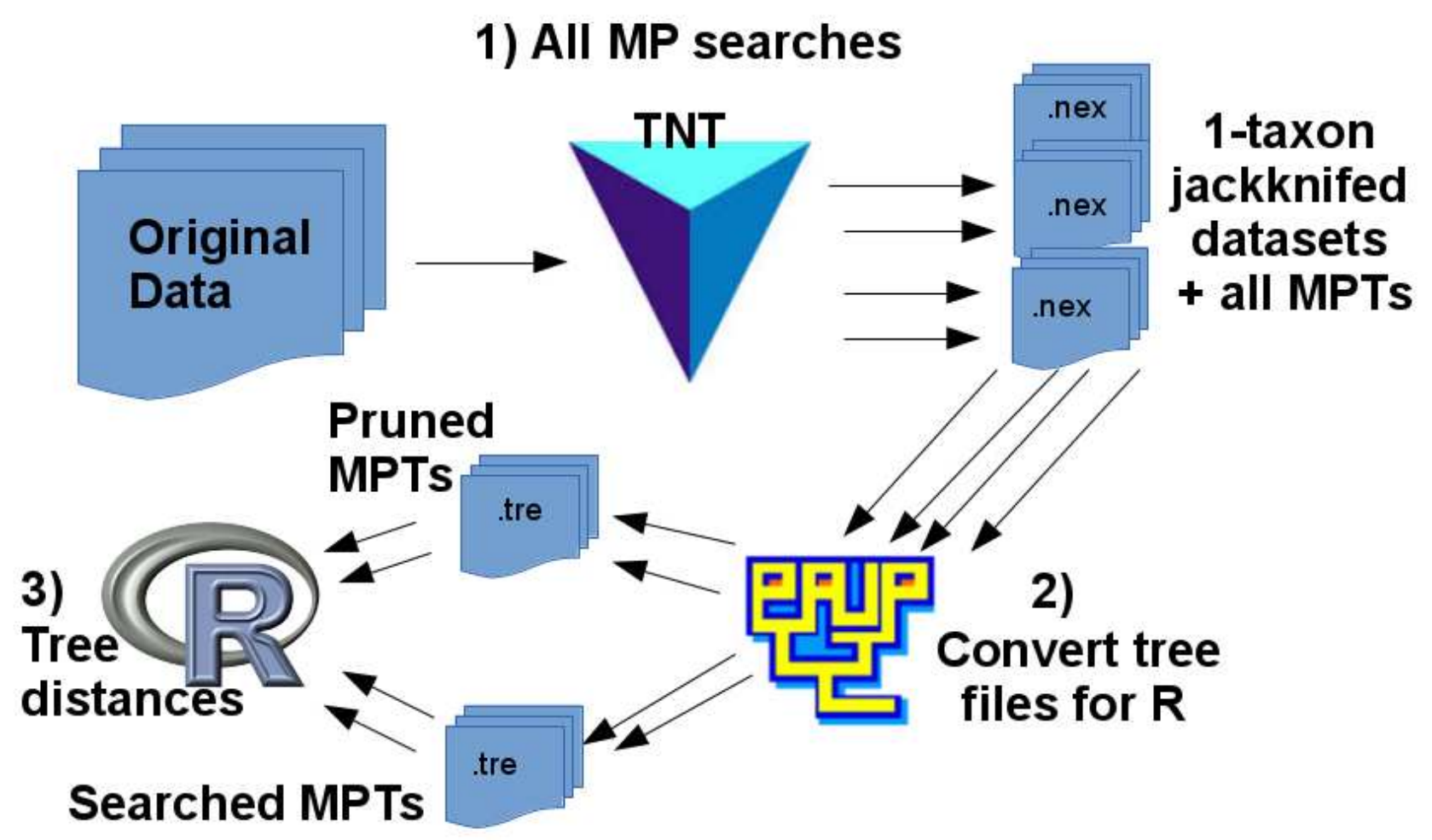

Figure 3.1 Schematic diagram of the workflow that implements taxon deletion

comparisons. In step 1 cladistic data matrices are analysed in TNT using New Technology searches (Nixon, 1999; Goloboff, 1999) to find all MPTs for the whole data sets, and all (non-root) single taxon jackknife variations. The results of these analyses, the matrices and the MPTs are exported to PAUP* for step 2 because R cannot directly import either of the tree export formats available natively in TNT. In step two the MPTs from the TNT analyses are simply converted to PHYLIP format with upto 10-character long taxon names. In step 3, the trees are loaded into R using the 'ape' package (Paradis et al. 2004) read.tree function and the mean minimum tree distances are found for each set of trees using the treedist function of the 'phangorn' package (Schliep 2011). Full code available online at: https://github.com/rossmounce/extinct_extant_chapter as well as on the $\mathrm{CD}$ that comes with the hard copy of this thesis.

This new analytical pipeline of first order taxon-jackknifing uses TNT (Goloboff et al., 2008) to perform all the maximum parsimony analyses using the original assumptions e.g. character ordering \& weighting that the original authors used. 
The output from $R$ ( $R$ Development Team, 2013) from each dataset is a plaintext tabseparated values file (.tsv) of the mean minimum RF (Robinson Foulds 1981) and PD distances (Zaretskii, 1965; Williams \& Clifford, 1971) for each deleted taxon, which aids further programmatic access and further command-line manipulation (Figure 1.). To summarize across all the 75 .tsv files programmatically, whilst minimizing humantranscription spreadsheet errors I made use of the 'ddply' function from the package plyr (Wickham, 2011) to help automate the process of creating the results summary Table 1.

\subsubsection{Leaf Stability Analyses}

Since Cobbett et al. (2007) significant computational advances have been made in both in tree searching (with TNT) and leaf stability calculation speed (with RogueNaRok; Aberer et al. 2013) allowing analyses to be performed on much larger data sets in reasonable times. Software now exists that can perform this type of analysis on trees of 116,334 taxa (Aberer et al 2013). For the leaf stability analyses reported here I used 200 bootstrap replicates, generated from initial analyses using New Technology searches at level 10 - Tree-Drifting, Sectorial searches, Tree-fusing (Nixon, 1999; Goloboff, 1999) on 100 random addition replicates. The 200 standard bootstrap replicates were calculated with 10 random replicates each. RogueNaRok was then used to calculate the leaf stabilities for each taxon. Statistical analyses of leaf support within data sets were performed in R using Mann-Whitney $\mathrm{U}$ tests (MWU). Analyses across data sets were performed in $\mathrm{R}$ using Wilcoxon matched pair tests, assuming that the null hypothesis is that there is no difference between fossil and Recent taxa. 


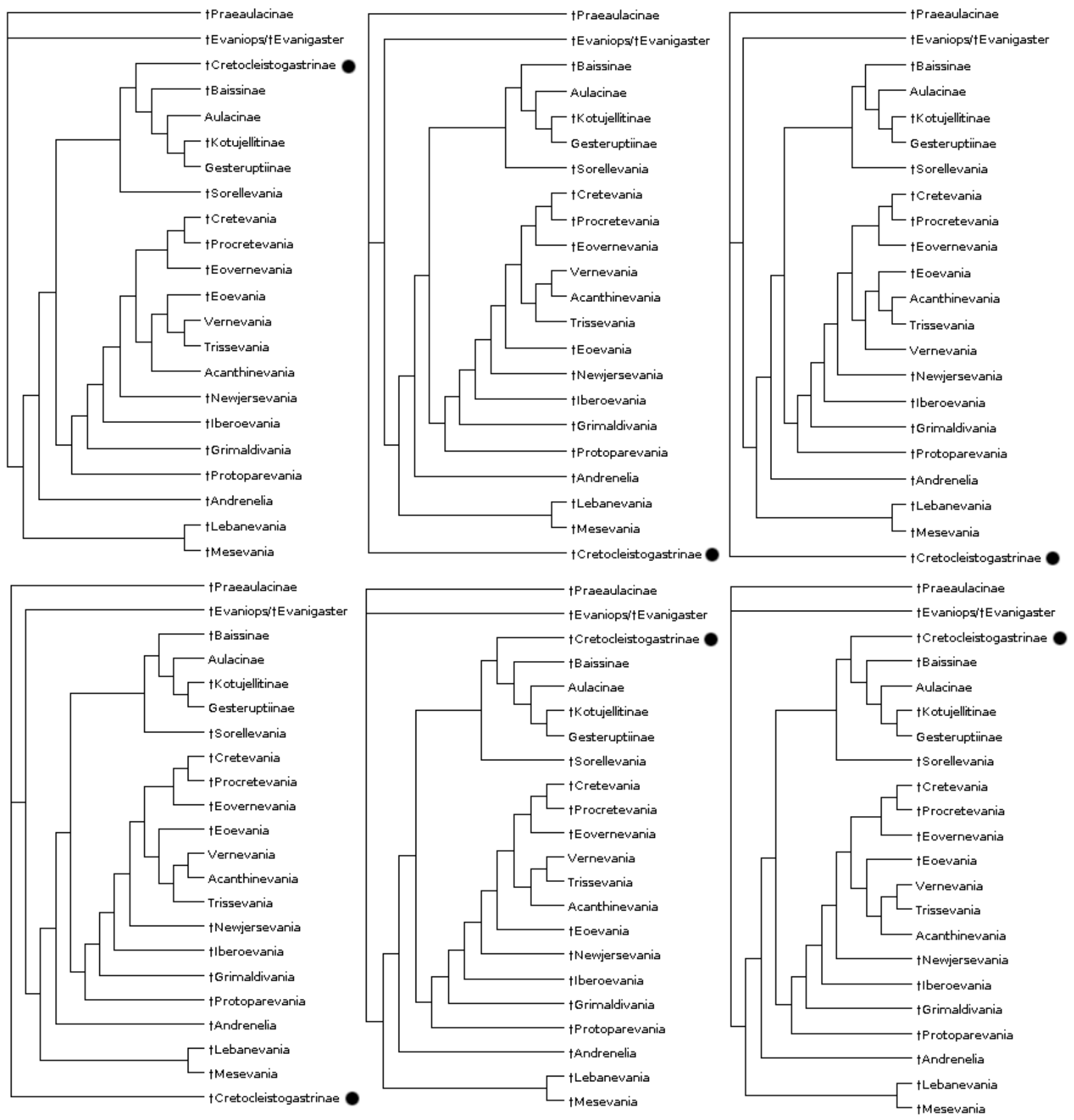

Figure 3.2 Demonstrating a taxon with low leaf-stability. These are all six MPTs from an analysis of the Evanioidea dataset by Penalver et al (2010). The extinct higher taxon Cretocleistogastrinae (indicated with a black circle) appears in two very different positions between the six cladograms. This taxon's leaf stability is 0.340 . If one excludes this taxon there is almost no difference between the leaf stability of the fossil (0.926) and extant (0.925) taxa in this dataset, on average. Fossil taxa are indicated by daggers $(\dagger)$. 


\subsection{Results}

All data, code and results are also available in a digitally re-usable form online under an OSI-approved open licence, to maximally enable re-use and re-analysis here on github: https://github.com/rossmounce/extinct_extant_chapter as well as on the CD which accompanies the hard copy deposit of this thesis.

Table 3.1 Summary statistics for the 75 morphological data sets analysed herein from vertebrate, invertebrate and botanical studies. Number of Fossil Taxa is the number of non-outgroup fossil taxa in the data set. Likewise Number of Extant Taxa is the number of non-outgroup extant taxa in the data set. Mean RF, Fossil describes the mean minimum Robinson Foulds distance between the MPT(s) in the original analysis and the MPT(s) from the fossil taxon-jackknifed analyses. Mean RF, Extant is the same but instead for when extant taxa are removed. Mean PD, Fossil describes the mean of all the mean minimum path length distances between the MPT(s) in the original analysis and the MPT(s) from the fossil taxon-jackknifed analyses. Mean PD, Extant is the mean of all the mean minimum path length distances between the MPT(s) from the original analysis and the extant taxon-jackknifed analyses. Mean Difference in MPTs measures the average effect of removing each taxon type on the number of MPTs found - a negative indicates that after removal of that taxon-type less MPTs were found on average, relative to the number of MPTs found in the original analysis. Mean Leaf Stability describes the average leaf stability of that taxon-type in the data set. It ranges from 0 which is unstable, to 1 is stable.

\begin{tabular}{|c|c|c|c|c|c|c|c|c|c|c|c|}
\hline \multirow[t]{2}{*}{ Dataset } & \multirow[t]{2}{*}{ 1st Author, Year } & \multirow{2}{*}{$\begin{array}{l}\text { No. } \\
\text { Fossil } \\
\text { Taxa }\end{array}$} & \multirow{2}{*}{$\begin{array}{l}\text { No. of } \\
\text { Extant } \\
\text { Taxa }\end{array}$} & \multicolumn{2}{|c|}{ Mean RF } & \multicolumn{2}{|c|}{ Mean PD } & \multicolumn{2}{|c|}{$\begin{array}{l}\text { Mean Diff. } \\
\text { MPTs }\end{array}$} & \multicolumn{2}{|c|}{$\begin{array}{c}\text { Mean Leaf } \\
\text { Stability }\end{array}$} \\
\hline & & & & Fossil & Extant & Fossil & Extant & Fossil & Extant & \begin{tabular}{|l} 
Fossi \\
1
\end{tabular} & Extant \\
\hline Acipenseriformes & Hilton, 2009 & 5 & 12 & 2.333 & 3.621 & 5.767 & 9.217 & -0.2 & 2.7 & .900 & .739 \\
\hline Adephaga & Beutel, 2012 & 17 & 30 & 5.298 & 7.045 & 21.413 & 25.514 & 49.4 & 153.7 & .996 & .970 \\
\hline Amniota & Hill, 2005 & 59 & 20 & 18.499 & 7.499 & 79.676 & 32.852 & 61.9 & 25.2 & .999 & 1.0 \\
\hline Archostemata & Beutel, 2008 & 8 & 16 & 6.333 & 6.219 & 13.948 & 15.496 & 1.3 & 0.7 & .996 & .991 \\
\hline Armadillos & Babot, 2012 & 13 & 9 & 8.994 & 10.183 & 21.509 & 23.082 & 4.1 & 4.8 & .996 & .995 \\
\hline Arthropods & Legg, 2012 & 98 & 74 & 7.380 & 8.053 & 52.794 & 49.599 & 0.39 & 0.2 & 1.0 & 1.0 \\
\hline Avian insectivores & Mayr, 2005 & 6 & 12 & 0.306 & 1.799 & 1.164 & 4.761 & -9.8 & 6.8 & .941 & .960 \\
\hline Bats & Simmons, 2008 & 5 & 23 & 1.600 & 1.510 & 5.875 & 5.033 & 1.8 & 1.6 & .992 & .984 \\
\hline Carnivora & Spaulding, 2012 & 35 & 15 & 7.968 & 8.394 & 39.135 & 33.594 & 44.3 & 207.3 & .983 & .988 \\
\hline Cetotheriidae & Fordyce, 2013 & 15 & 7 & 2.267 & 2.524 & 5.847 & 7.200 & 0.8 & 0.7 & .996 & .983 \\
\hline Chitons & Sigwart, 2007 & 26 & 7 & 15.153 & 21.238 & 40.054 & 52.821 & 64.8 & 119.6 & .971 & .960 \\
\hline Clawed lobsters & Ahyong, 2006 & 12 & 15 & 4.275 & 4.542 & 12.012 & 13.387 & 11.3 & 1.1 & .966 & .983 \\
\hline Coliiform birds & Ksepka, 2010 & 11 & 16 & 6.887 & 3.854 & 18.233 & 10.077 & 39 & 2.8 & .974 & .973 \\
\hline Crabs & Karasawa, 2006 & 4 & 40 & 7.636 & 2.292 & 27.577 & 12.592 & 5.0 & 1.8 & .997 & .992 \\
\hline Crocodiles & Puertolas, 2011 & 11 & 39 & 3.273 & 2.886 & 16.097 & 14.426 & 10.5 & 4.5 & .994 & .997 \\
\hline
\end{tabular}




\begin{tabular}{|c|c|c|c|c|c|c|c|c|c|c|c|}
\hline Echinoids & Mihaljevic, 2011 & 6 & 21 & 1.238 & 6.226 & 4.984 & 12.211 & -0.9 & 12.2 & .957 & .979 \\
\hline Ensign scale insects & Vea, 2012 & 8 & 38 & 3.663 & 3.785 & 15.215 & 15.713 & 680.9 & 187.5 & .988 & .986 \\
\hline Euarchonta & Bloch, 2007 & 16 & 4 & 3.971 & 0.500 & 8.918 & 1.785 & 3.1 & 0.0 & .963 & .926 \\
\hline Evanioidea & Penalver, 2010 & 16 & 5 & 4.841 & 1.800 & 11.329 & 4.495 & 7.4 & 4.8 & .889 & .929 \\
\hline Flatfish & Friedman, 2008 & 5 & 13 & 3.133 & 3.388 & 7.375 & 7.637 & 0.4 & 3.8 & .973 & .950 \\
\hline Frogs & Trueb, 2006 & 12 & 8 & 1.435 & 1.146 & 4.085 & 3.574 & 2.1 & 0.8 & .975 & .965 \\
\hline Gnoristinae & Blagoderov, 2004 & 22 & 17 & 13.638 & 14.310 & 45.494 & 45.885 & 27.5 & 28.8 & .958 & .956 \\
\hline Gonorynchiforms & Poyato-Ariza, 2010 & 17 & 9 & 4.177 & 7.092 & 11.046 & 16.272 & 21.2 & 99.3 & .880 & .892 \\
\hline Harvestmen & Garwood, 2011 & 3 & 40 & 5.675 & 8.066 & 18.448 & 24.559 & -57.7 & -9.2 & .870 & .978 \\
\hline Horned Crocs & Brochu, 2010 & 21 & 15 & 7.427 & 7.136 & 18.555 & -61.762 & -61.8 & -4.5 & .968 & .966 \\
\hline Junglandaceae & Manos, 2007 & 5 & 21 & 6.871 & 4.045 & 16.944 & 11.040 & -10.0 & 2.7 & .963 & .958 \\
\hline Kangaroos & Prideaux, 2010 & 17 & 17 & 2.094 & 2.853 & 11.221 & 14.713 & 0.3 & 0.5 & .996 & .990 \\
\hline Lagomorpha & Asher, 2005 & 38 & 28 & 9.312 & 12.266 & 36.614 & 45.862 & 44.9 & 64.6 & .992 & .995 \\
\hline Lepidosauromorpha & Li, 2007 & 10 & 23 & 2.600 & 2.186 & 7.922 & 7.215 & 0.6 & 2.8 & .959 & .971 \\
\hline Mancallinae & Smith, 2011 & 6 & 52 & 7.347 & 9.910 & 30.602 & 44.865 & 7.0 & 54.8 & .994 & .994 \\
\hline Megalyridae wasps & Vilhelmsen, 2010 & 14 & 14 & 1.730 & 3.428 & 4.816 & 11.621 & -475.4 & -189.6 & .595 & .564 \\
\hline Mysticetes & Bisconti, 2008 & 22 & 12 & 3.018 & 2.779 & 11.454 & 10.424 & 1.3 & -0.5 & .957 & .959 \\
\hline Mysticeti & Bouetel, 2006 & 15 & 7 & 1.969 & 0.371 & 0.371 & 1.349 & -6.5 & -9.1 & .975 & .971 \\
\hline Neoteleosts & Dietze, 2009 & 6 & 10 & 4.768 & 3.652 & 10.456 & 8.195 & 3.5 & 2.9 & .961 & .958 \\
\hline Odontoceti & Lambert, 2013 & 21 & 6 & 4.584 & 7.079 & 12.715 & 19.018 & 2.8 & 22.5 & .973 & .990 \\
\hline Osteoglossomorpha & Zhang, 2006 & 19 & 11 & 2.604 & 0.766 & 8.240 & 2.386 & 6.9 & 2.0 & .994 & .996 \\
\hline Osteoglossomorpha & GuangHui, 2009 & 4 & 11 & 4.500 & 5.561 & 8.088 & 0.455 & 0.3 & 1.5 & .981 & .962 \\
\hline Pan-Apodiformes & Ksepka, 2013 & 14 & 13 & 4.298 & 5.769 & 14.859 & 18.281 & 4.3 & 3.5 & .992 & .985 \\
\hline Pangolins & Gaudin, 2009 & 8 & 8 & 3.000 & 2.583 & 6.315 & 5.460 & 0.6 & -0.5 & .965 & .970 \\
\hline Papionin primates & Gilbert, 2013 & 16 & 7 & 8.002 & 23.357 & 16.062 & 43.998 & -2.8 & -4.0 & .957 & .940 \\
\hline Pelagornithidae & Mayr, 2011b & 5 & 19 & 3.100 & 4.229 & 8.841 & 9.985 & 2.2 & 1.7 & .881 & .946 \\
\hline Pelecaniformes & Smith, 2010 & 8 & 50 & 4.565 & 1.835 & 20.645 & 9.614 & 0.4 & 1.1 & .999 & .985 \\
\hline Penguins & Hospitaleche, 2007 & 5 & 16 & 6.300 & 7.286 & 12.313 & 16.371 & 0.6 & 2.9 & .990 & .980 \\
\hline Percomorp & Whitlock, 2010 & 4 & 22 & 3.558 & 2.346 & 10.972 & 7.429 & 2.8 & 0.8 & .875 & .838 \\
\hline Pinaceae & Klymiuk, 2012 & 40 & 11 & 65.888 & 75.571 & 199.127 & 229.889 & 1458.2 & 2002.7 & .826 & .996 \\
\hline Pinnipeds & Boessenecker, 2013 & 17 & 5 & 1.739 & 1.460 & 6.595 & 6.366 & -7.8 & -8.4 & .922 & .882 \\
\hline Placental Mammals & O'Leary, 2013 & 39 & 46 & 10.195 & 8.401 & 45.343 & 36.985 & 0.8 & 1.13 & .999 & 1.0 \\
\hline Placental Mammals & Luo, 2011 & 59 & 11 & 8.360 & 18.960 & 37.783 & 71.396 & 14.5 & 18.1 & .996 & .998 \\
\hline Podocarpaceae & Greenwood, 2013 & 5 & 21 & 6.765 & 9.183 & 17.416 & 21.079 & 13.0 & 8.5 & .865 & .904 \\
\hline Brachyura & Karasawa, 2011 & 22 & 14 & 8.716 & 8.369 & 32.442 & 36.470 & 2.9 & 1.1 & .996 & .998 \\
\hline Polyphaga & Fikacek, 2012 & 10 & 29 & 10.390 & 15.111 & 27.863 & 36.878 & 549.0 & 959.0 & .910 & .950 \\
\hline Porpoises & Lambert, 2008 & 9 & 6 & 3.932 & 0.167 & 8.859 & 0.553 & 4.9 & -0.8 & .973 & .990 \\
\hline Primates & Beard, 2009 & 34 & 4 & 3.623 & 3.500 & 12.608 & 13.952 & 1.1 & -4.0 & .976 & .975 \\
\hline Psittaciformes & Mayr, 2010 & 4 & 29 & 18.611 & 18.460 & 44.349 & 46.074 & 644.2 & 895.5 & .861 & .948 \\
\hline Pycnogida & Arango, 2007 & 4 & 65 & 38.272 & 24.975 & 173.951 & 96.312 & 1697.0 & 467.1 & .937 & .990 \\
\hline Ratites & Bourdon, 2009 & 9 & 6 & 0.000 & 0.000 & 0.000 & 0.000 & 0.0 & 0.0 & .972 & .969 \\
\hline Ratites & Worthy, 2012 & 11 & 14 & 1.916 & 0.143 & 5.999 & 0.655 & 0.3 & -0.2 & .979 & .985 \\
\hline Rhinos & Deng, 2008 & 26 & 5 & 2.123 & 1.257 & 7.903 & 3.483 & -22.1 & -21.6 & .933 & .874 \\
\hline
\end{tabular}




\begin{tabular}{|l|l|r|r|r|r|r|r|r|r|r|r|}
\hline Rhizomyinae & López-Antoñanzas, 2013 & 32 & 6 & 10.115 & 4.551 & 27.585 & 14.389 & 216.3 & 0.0 & .911 & .956 \\
\hline Salamanders & Skutchas, 2012 & 11 & 10 & 9.101 & 6.313 & 16.210 & 12.567 & 12.1 & 5.7 & .941 & .920 \\
\hline Sciaroidea & Blagoderov, 2009 & 7 & 10 & 0.714 & 1.117 & 2.176 & 2.791 & 0.3 & -0.2 & .926 & .961 \\
\hline Sharks & Klug, 2010 & 11 & 18 & 5.742 & 7.525 & 15.165 & 18.164 & 0.7 & 16.9 & .909 & .973 \\
\hline Sharks & Pradel, 2011 & 15 & 3 & 2.513 & 0.333 & 6.267 & 0.943 & 0.8 & 0.3 & .941 & .941 \\
\hline Side-necked turtles & Gaffney, 2011 & 27 & 9 & 2.309 & 2.900 & 10.663 & 13.472 & 0.4 & 0.8 & .993 & .987 \\
\hline Snakes & Apesteguia, 2006 & 6 & 12 & 1.667 & 4.500 & 4.387 & 8.981 & -0.5 & 1.7 & .901 & .904 \\
\hline Squaliform sharks & Adnet, 2001 & 7 & 16 & 6.896 & 4.875 & 18.701 & 14.127 & 36.4 & 2.2 & .979 & .988 \\
\hline Squamata & Hutchinson, 2012 & 11 & 24 & 1.431 & 2.349 & 5.455 & 6.863 & -5.4 & 15.3 & .953 & .984 \\
\hline Stem Rollers & Clarke, 2009 & 5 & 42 & 5.510 & 6.050 & 22.510 & 22.405 & 20.8 & 69.9 & .956 & .994 \\
\hline Stingrays & Claeson, 2010 & 12 & 27 & 17.295 & 10.261 & 54.495 & 34.295 & 1114.5 & 255.3 & .978 & .969 \\
\hline Teleosts & Diogo, 2008 & 5 & 64 & 2.333 & 3.700 & 11.623 & 18.355 & 0.0 & 9.7 & .992 & .993 \\
\hline Teleosts & Hurley, 2007 & 22 & 6 & 1.496 & 1.805 & 5.995 & 6.523 & -5.7 & 10.5 & .923 & .937 \\
\hline Tetrapods & Diogo, 2007 & 7 & 73 & 1.683 & 4.242 & 12.416 & 21.768 & 18.3 & 77.4 & .999 & .999 \\
\hline Turtles & Joyce, 2007 & 40 & 22 & 7.295 & 7.312 & 32.970 & 36.245 & 57.1 & 24.0 & .973 & .977 \\
\hline Wasps & Perrichot, 2009 & 7 & 9 & 1.920 & 2.796 & 4.694 & 6.478 & -3.7 & -14.0 & .856 & .861 \\
\hline Waterfowl & Worthy, 2009 & 10 & 51 & 5.224 & 7.845 & 18.021 & 29.677 & 26.8 & 61.6 & .994 & .994 \\
\hline & & & & & & \\
\hline
\end{tabular}

\subsubsection{On average Fossil and Extant Taxa have remarkably similar Topological Impact as judged by single-taxon deletions}

Across the 75 data sets the mean of the mean minimum RF distances for fossil taxa is 6.58, and 6.77 for extant taxa. The mean of the mean minimum PD distances for fossil taxa is 22.02 and 21.64 for extant taxa. Wilcoxon tests indicated that extant and fossil taxa are not significantly different in either mean minimum RF distance $(P=0.941,5 \%$ significance level) or mean minimum PD distance $(P=0.9985,5 \%$ significance level). Nor was there a difference in the standard deviation of their mean minimum distances, $R F(P=$ $0.5438,5 \%$ significance level), PD ( $P=0.6587,5 \%$ significance level).

Subsequent examination of the within dataset differences using Mann Whitney $U$ tests show that even where the two group means look different e.g. RF distances this tends to be caused by exceptional individual taxa rather than any general properties of each class of taxa (see data supplied online / on CD; figure 3.3). 

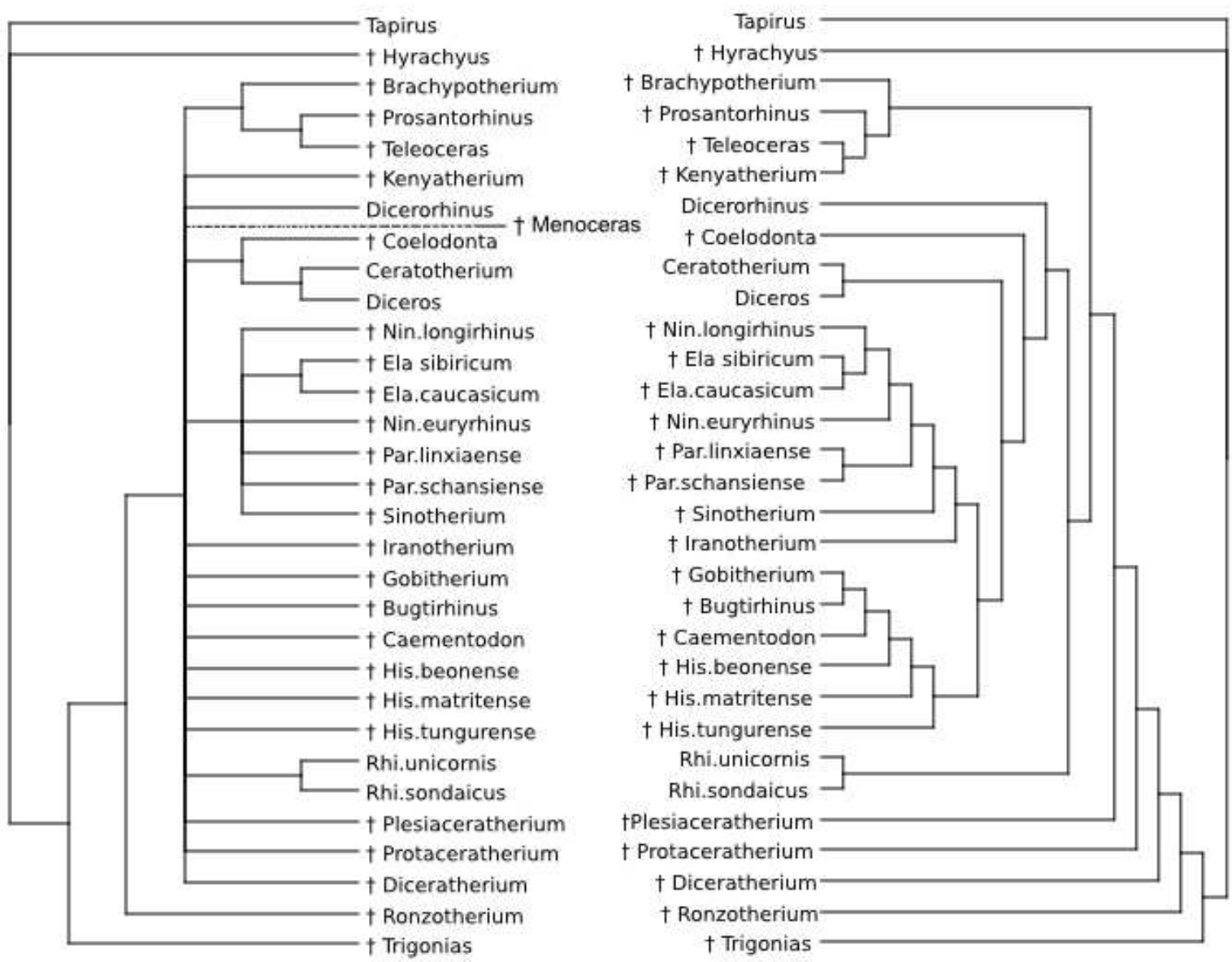

Figure 3.3 Measuring the relationships impact of removing single taxa. Data from Rhinocerotidae (Deng, 2008). The tree on the left is the pruned strict consensus tree. Data set analyzed with Menoceras included, yields 37 MPTs. The location of Menoceras in the original tree is indicated by the dashed line. The tree on the right is the Searched tree from the same data matrix without Menoceras which yields just one MPT. The symmetric difference between these two trees is 6, whilst the path difference is 150 .

\subsubsection{Fossil Taxa on average do not increase the number of MPTs any more than Extant Taxa}

Averaging across all the data sets for the difference in numbers of MPTs upon removal of fossil or extant taxa, there was no significant difference in change in the number of MPTs as assessed with a Wilcoxon matched-pairs test $(P=0.554,5 \%$ significance level), similarly no significant difference between their standard deviations either $(P=0.8252,5 \%$ significance level). There is a strong, and significant positive correlation between the 
minimum meanRF and minimum meanPD distances $\left(R=0.96, P=2.2 \times 10^{-16}\right.$, Pearson's Product-Moment Correlation Coefficient). Inclusion of fossil taxa demonstrated both negative (figure 3.3) and postive effects on the number of MPTs (figure 3.4).
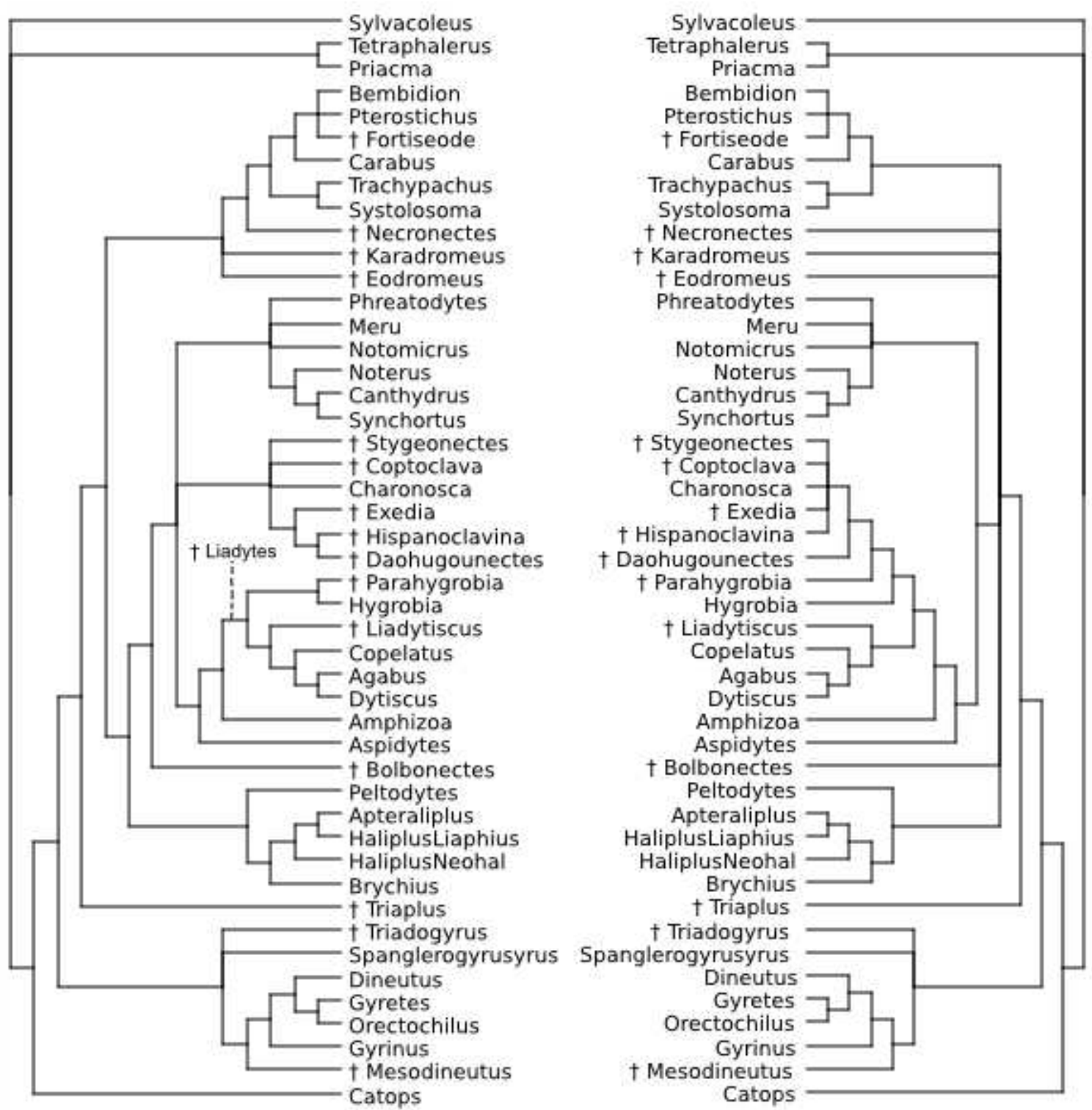

Figure 3.4 A demonstration of a fossil taxon that helps 'support' a phylogeny; in this case the removal of Liadytes causes deleterious effects on the resolution (data from Beutel et al. 2012). There are less fully resolved nodes on the right (the taxon deleted tree), than on the right (the original whole dataset analysis). 


\subsubsection{Leaf Stability}

30 data sets had a higher mean fossil leaf stability than the mean extant leaf stability. This proportion of trials (30/75) suggests there is no significant difference in leaf stability between fossils and extant taxa (binomial test, $P=0.1053,5 \%$ significance level). It should be noted that the difference in mean leaf stability between extant and fossil was often very slight, with an average absolute difference between the means of just 0.021 .

\subsubsection{Extreme Taxa}

For max.RF (see Appendix 3.2) extant taxa had the highest meanRF distance of all taxa in 41 of the 75 data sets. For max.PD extant taxa had the highest meanPD distance of all taxa in just 37 of the 75 data sets. On an individual taxon-level, the removal of extant taxa caused the highest increase in MPTs in 38 out of the 75 data sets. Using a simple binomial test none of these 'winning' frequencies are significant $(P=0.488, P=1$ and $P=1$ respectively).

73 of the data sets had one or more taxa whose deletion increased the number of MPTs relative to an analysis of the 'full' dataset. Only Deng (2008; figure 3.3) and Bourdon (2009) had data whereby the deletion of any single non-root taxon resulted in either the same number or less than the original number of MPTs. 56 data sets had more than half of their non-root taxa cause an increase in MPTs if deleted $-a$ binomial test shows this to be significant $\left(P=2.2 \times 10^{-5}\right)$. As a percentage of the total fossil taxa in a dataset, on average $35.8 \%$ of fossil taxa caused an increase in MPTs. As a percentage of total extant taxa in a dataset, on average $36.1 \%$ of extant taxa caused an increase in MPTs (see Appendix 3.2). 


\subsection{Discussion}

These new results clearly support those first reported by Cobbett et al. (2007). Fossil and extant taxa on average have a similar per taxon impact on inferred relationships. These results also support the notion that fossil taxa are no more different in their effect on the resolution of the strict consensus that extant taxa, as also reported by Cobbett et al. (2007). However, in this study I obtained a rather different result with respect to leaf stability, than the results of Cobbett et al. (2007). In their sample of 36 datasets tested for leaf stability with RadCon (Thorley and Page, 2000) they detected a significant difference between fossil and extant taxa on leaf stability, with fossils tending to be significantly more unstable. In this analysis using RogueNaRok (Aberer et al. 2013) which calculates leaf stability slightly differently - using rooted triplets, I have found no significant difference between extant and fossil taxa for leaf stability although the $p$-value is quite low $(P=0.105)$.

The biggest advance this chapter offers however, is the code pipeline, which allows any investigator to test their own data sets for these properties. It is sufficiently generalised to accept most data, and uses the common tools that many systematists use, namely PAUP*, TNT and R. The new workflow is much quicker all in all relative to the DELBAT/DELSUM basic scripts of Cobbett et al. (2007) and offers easy flexibility and extensibility through the use of $R$.

\subsection{Conclusion}

Based on the results presented here and in Cobbett et al. (2007), it should now be clear that there is no justification to a priori exclude fossils from parsimony analyses of morphology to infer phylogeny. There are a multitude of possible benefits from their inclusion, and multiple different tests available to help one decide a posteriori if 'rogue' or 'wildcard' taxa are causing problems in an analysis (Wilkinson 1995b; Thorley and Page 2000; Smith \& Dunn, 2008; Pol and Escapa 2009; Mariadassou et al. 2012; Aberer et al. 2013). There are two avenues of future research I would like to explore arising from this work. The first is a comparative methods review between all the various different tests of taxon stability \& inclusion/exclusion criteria. It is unclear to me from the literature which 
one of these might be optimal for parsimony analyses of morphology. What are the relative strengths and weaknesses of these methods? Do they all agree for all datasets? If they differ, on what basis do they differ - character evidence or relationship resolution evidence? The second is to extend the current analytical pipeline used in this chapter so that it tests a wider range of tree to tree distance measures. I have already been in contact with the lead author of TreeCmp (Bogdanowicz et al. 2012) to see how I can integrate his program into my workflow. Once integrated, I would be able to test additional metrics such as matching clusters, nodal splits, quartets, triplets and agreement subtrees. 


\section{Chapter 4: A review of the ILD \\ randomisation test: uses and abuses}

\subsection{Abstract}

The incongruence length difference (ILD) randomisation test of Farris et al.. (1995a, $1995 b$ ) is often applied to systematic data sets comprising qualitatively or logically distinct data partitions (e.g., molecules and morphology, different loci). It has variously been used to assess phylogenetic accuracy (1), 'data combinability' (2), difference in evolutionary history (3) and difference in evolutionary rate (4). Several authors have noted that the ILD test is not designed to address all of these issues, particularly the first two, and that the inferences drawn from test results are often questionable. Here, I quantitatively review the usage of the ILD test in over 250 papers published between 2009 and 2010 - an exhaustive sampling of the papers that I had automated harvesting access to. There appears to be very little consensus on how best to implement the ILD test appropriately. There is often no justification for the number of replications used, or for the search parameters specified. In many cases, the settings used are not reported at all, making it impossible to reproduce the results exactly. Where p-values are not reported, results cannot be re-validated. I conclude with a concise summary re-iterating what is already known about the proper usage of the test and suggest minor extensions that may reveal extra useful information from the test performed. 


\subsection{Introduction}

In 1981, Mickevitch and Farris introduced a new quantitative measure of incongruence of phylogenetic signal between data partitions, which I shall refer to as the 'MF index' to avoid confusion. They were examining the difference in signal between allozyme data and morphometric characters for the phylogeny of Menidia and demonstrated that the withindata partition incongruence was greater than the between-data partition incongruence $(p$ 366 - 367). The idea of examining incongruence between data partitions in this manner was then not improved upon until 1991 when Farris further developed the MF index into a proper statistical test with the addition of a randomisation process and an implementation in his arn program, presented at the Willi Hennig Society meeting that year (Farris et al. 1995a).

For two data partitions, $A$ and $B$, the MF index is: $L_{A B}-\left(L_{A}+L_{B}\right)$ where $L_{A B}$ is the optimal tree length in steps of the simultaneous Maximum Parsimony (MP) analysis of both data sources together - the total evidence analysis, $L_{A}$ is the optimal tree length of an MP analysis of just data $A$, and likewise $L_{B}$ is the optimal tree length of an MP analysis of just data $B$. If the data partitions have low incongruence between each other then $\left(L_{A}+L_{B}\right)$ will be expected to be only marginally smaller than $L_{A B}$. Whereas if $\left(L_{A}+L_{B}\right)$ is much smaller than $L_{A B}$, then this difference in tree length must be caused by incongruence between $A$ and $B$. The extra steps required to fit the combined $A B$ data to the optimal tree $L_{A B}$ are referred to as homoplasy and neither the ILD test, nor the MF index help distinguish further what type or cause of incongruence - just that to some extent (or not at all if no difference), that there is some incongruence of signal between the data partitions.

The difference between the MF index and the ILD test, is that the latter is an extension that critically examines the statistical significance of the MF index relative to a randomly sampled null distribution of possible MF indices for data partitions of the same matrix parameters and character-state composition as data A and data B. ILD test scores are thus much more comparable between different studies than simple MF indices alone. 


\subsubsection{Historical Importance and Context}

Any sensible discussion and interpretation of the history, development and usage of the ILD test must take account of the historical context in which it was developed - there are a number of background factors and themes of which I shall expand upon in this section that are of great relevance to the understanding of the usage of the ILD test.

The ILD test (Farris et al. 1995b) was introduced over a decade before the first conference on phylogenomics (in 2006; Philippe \& Blanchette, 2007); at a time when the variety of molecular sequence data available simply wasn't anywhere near as bountiful as it is today. This was a time when good molecular phylogenetic papers published in respected phylogenetic journals presented data from just one or two loci (e.g. Jacobs et al 1995; Freeman \& Zink 1995; Domanico \& Phillips 1995; Myers et al 1995; Rosel et al 1995). It was also right in the midst of a long-running controversy and debate around the 'combinability' of different data sources (Swofford, 1991, p327 "To Combine or Not to Combine"; Siddall 1997), sometimes played-out as taxonomic congruence (e.g. Miyamoto \& Finch, 1995) "vs" the total evidence approach (Kluge, 1989; Eernisse \& Kluge, 1993), and more specialised cases such as "morphology vs molecules" in phylogenetic inference (Wheeler, 1991; Swofford, 1991; Hedges \& Maxson, 1996).

Early efforts such as Bull et al. (1993; fig. 2) used simulations to provide evidence that combining different data sets in an analysis can provide a 'worse' overall estimate of phylogeny than analyzing data sets separately. Thus many systematists were genuinely unsure of whether to combine or not to combine their data, despite the clear philosophical superiority of using as much relevant evidence as possible - the Total Evidence approach (Kluge, 1989). Even up to the present day both taxonomic congruence and total evidence approaches are still both being actively used, developed and debated: STEM (Kubatko et al, 2009) and *BEAST (Heled \& Drummond, 2010) are popular programs that separately infer gene trees to help form a consensus estimate of the overarching species tree (a form of the taxonomic congruence approach). Simultaneously, many papers continue to pursue total evidence (concatenation) methods particularly those that choose to incorporate molecular and morphological data (e.g. Finarelli 2008; Jenner et al 2009; Pretti et al 2009; Schuh et al 2009; Zrzavy et al 2009; Pepato et al 2010; Prevosti 2010; Davis 2010; Fritz et al 2011; Lehtonen et al 2011; Lopardo et al 2011; Carasco et al 2012; Clennett et al 2012; Janssens et al 2012; Ronquist et al 2012; Wood et al 2012) 
Thus in recent times the only thing that has changed is the terms by which the debate is framed; instead of taxonomic congruence versus total evidence, debate now centers upon 'coalescence' versus 'concatenation' methods (e.g. sensu Kubatko \& Degnan, 2007) which although slightly more specific, clearly has roots in this old debate.

\subsubsection{Inappropriate usages of the ILD test}

After publication of the ILD test procedure there appeared to be much confusion and uncertainty about what the ILD test could be used for. This led to a multitude of critiques of these various uses (Cunningham 1997b; Yoder et al. 2001; Barker \& Lutzoni 2002; Dowton \& Austin, 2002; Darlu \& Lecointre 2002) and led some to question, particularly Barker \& Lutzoni (2002) whether the ILD test had any valid use at all.

Farris et al. (1995a) contains no discernable suggestion that the ILD test could be used to decide whether to combine or not to combine data - it merely describes the test as a measure of the significance of incongruence between data partitions. Yet after its publication some researchers started to use the ILD test for this very purpose - as a test of "combinability" (e.g. Johnson \& Sorensen 1998; Vidal \& Lecointre 1998; Carbonne et al 1999; Hoot et al 1999; Spangler and Olmstead 1999), most probably because early versions of the popular PAUP software (versions before 3.1) by Swofford referred to the ILD test as a "combinability" test (Farris 1997; Yoder et al 2001). Other computational implementations of the ILD test such as Siddall's ARNIE and HARDARN, part of the Random Cladistics package (reviewed in Allard et al, 1999b) contain no such assertions about "combinability". These implementations however, appear to have been much less used than PAUP and thus I speculate that the use of the ILD test to decide "combinability" probably originated from this early PAUP implementation. This incorrect usage of the ILD test as a measure of combinability is discussed and rightly dismissed in Yoder et al (2001) and Barker \& Lutzoni (2002). However, neither Yoder et al (2001) nor Barker \& Lutzoni (2002) cite Farris' much earlier denouncing of this usage in a conference talk (in 1996) \& the subsequently published abstract of that talk (Farris, 1997) in which it is stated that: "incongruence and non-combinability are not the same thing. The idea of testing for noncombinability seems questionable at best".

Similarly some researchers appear to have initially used the ILD test as a measure of 
'phylogenetic accuracy' Cunningham (1997b), or 'topological congruence' (Barker \& Lutzoni 2002). The logic behind these uses is difficult to understand given the construction of the test (Mickevitch \& Farris 1981; Farris 1995a) - the ILD test is calculated using tree length and character congruence NOT tree topology or topological congruence (but see the Topological Incongruence Length Difference [TILD] of Wheeler, 1999 for a measure that does directly test topological congruence). Finally, despite some early criticism (Dowton \& Austin 2002), some promise has been shown for using Wheeler's (1999) derivative of the ILD for data exploration (sensu Grant \& Kluge 2003), specifically for use in mixed parameter sensitivity analyses (e.g. Sharma et al. 2011).

I agree with Hipp et al. (2004), contra Yoder et al (2001) and contra Barker \& Lutzoni (2002) that the ILD remains a useful and valid test to assess global partition character congruence and that this is distinct from the inappropriate uses of measuring 'phylogenetic accuracy' or 'topological congruence'. Planet's (2006) comprensive review appears to agree with such usage of the ILD test.

\subsubsection{Additional developments}

Aside from the many different uses the ILD test was used for; there are a number of other short points about the ILD test that I should note here for the sake of completeness. Sullivan (1996), Cunningham (1997a) and others have suggested that 0.05 may be too liberal a significance threshold to use for the ILD test, hence in the literature sometimes smaller significance thresholds such as 0.01 have been used.

Cunningham (1997b) \& Lee (2001) note that invariant and parsimony-uninformative characters should be excluded prior to ILD tests. Lee (2001) demonstrates that such phylogenetically-uniformative characters, if unevenly distributed between partitions can artificially decrease the ILD p-value, sometimes creating false 'significance' - entirely due to the presence of these uninformative characters. My only comment on this is that it is not really much of a "problem" as indicated by Ramirez (2006). Many other cladistic calculations e.g. the ensemble consistency index ( $\mathrm{Cl}$; Kluge \& Farris 1969) require uninformative characters to be excluded and thus this is a standard procedure in most analytical workflows.

Ramirez's (2006) paper contains novel criticisms of the ILD test, for example that localizing exactly where incongruence is with the ILD test is difficult; that the available "strategies are 
only feasible for small data sets" - the computational power available now in 2013 makes ILD tests, and taxon-jackknife ILD tests quite feasible even for data sets comprising of many hundreds of terminals. Ramirez (2006) also notes that ILD test results can 'conflict' with partitioned Bremer support results - but this is because the two tests are measuring fundamentally different things; partitioned character support at nodes (local) versus the congruence of entire data partitions (global). These methods should be viewed as complementary and do not give exactly the same information.

Ramirez (2006) figure 2 shows that duplicating taxa lead to undesirable ILD test results. But I question if this is a realistic example. As with uninformative characters, if a cladistic matrix contains uninformative or completely duplicative taxa these are usually removed prior to analysis with 'Safe Taxonomic Reduction' as there is little point including them in the analysis if they include no new phylogenetic information (Wilkinson 1995).

Finally, the main novel critique that Ramirez (2006) presents: "hypercongruence" seems to me to confuse topological congruence and character congruence. Ramirez (2006) figure 4 presents an excellent example of why character congruence and topological congruence are not the same thing. Whilst Ramirez (2006) presents this difference as a problem of the ILD test, I instead suggest interested readers carefully examine the character matrices to rationalize the ILD results therein presented.

Dolphin et al. (2000) present an interesting additional procedure that one can choose to perform to help disambiguate between the incongruence due to 'noise' and the 'real' incongruence due to conflicting phylogenetic signals. Despite being well-cited (over 200 times according to Google Scholar), few researchers appear to have chosen to actually implement this additional procedure. Quicke et al (2007) boldly propose that such noise imbalances can be simply 'corrected' for, on the basis of the simulations they run in their paper. However, their arcsine-transformed ILD-metric does not convince me because I do not believe the simulated data matrices they analysed are biologically similar to real molecular or morphological character data. Their method of representing trees as unambiguous binary characters supporting each node, bears little relation to the complexity of empirical character data sets, and furthermore the calculation of RI depends on the number of states allowed per character (Hoyal Cuthill et al 2010) - this is much more variable than just binary in empirical data sets. Thus I do not think their results are necessarily transferrable to real empirical data. Quicke et al (2007) has relatively few citations so far (about 24 according to Google Scholar), and of those almost none of these 
actually implement the suggested arcsine-transformed ILD test.

\subsubsection{Raison d'etre for this review of ILD test usage}

Given the long and complicated history of the ILD test, the various debates and usages of it - I thought it might be instructive to see how people are using the ILD test now. Given how many papers all the salient info is spread across one can hardly blame authors for mis-applying or mis-reporting the ILD test, but prior to writing this chapter I had noticed a few odd ones, which partially spurred this review. In doing this review I hope to highlight common errors and misperceptions so that future usage of the ILD test by the research community can be improved, standardised and made more re-usable for comparative cladistic analyses e.g. (Fisher-Reid \& Wiens 2011).

\subsection{Methods}

\section{Literature Search}

I performed a literature search for papers published in the year 2009 or 2010, which cite Farris et al. (1995b) using the Thompson Reuters Web of Science 'SCI-EXPANDED' database which returned 443 articles. I exported the bibliographic data corresponding to these 443 articles to a bibtex file which is available online for re-use \& validation (https://github.com/rossmounce/thesis_ESM/blob/master/ILD_chapter/443_ILD_citing_pap ers.bib). I then passed Paperpile (http://paperpile.com/) this bibtex file to help automatically harvest and download the corresponding full-text PDF files for as many of the 443 articles as I had legitimate access to through University of Bath journal subscriptions. After this process I had access to corresponding full-text for 278 of the 443 articles. I was thus apparently unable to access $37 \%$ (165) of the papers found by this particular literature search. Reasons for my inability to automatically harvest corresponding full-text for some articles included:

- lack of subscription access through my university (e.g. articles from subscription journals such as Taxon, Journal of Bryology, Auk, Invertebrate Systematics, etc...)

- $\quad$ poor / incomplete source bibiographic data from Web of Science that Paperpile could not automatically locate a corresponding article for 
- Articles from smaller, more unusual publishers for which Paperpile could not automatically harvest full-text articles from

For the purpose of this analysis 278 articles, whilst not the entire sample for the time period assessed, is certainly a large enough sample, from a representative variety of journals (84) and authors from which to draw conclusions from. It was thus not deemed worth manually trying to obtain full-text copies of the remaining 165 papers. The 278 automatically harvested would suffice.

The distribution of the 278 full-text articles across the 84 different journals was remarkably non-random; over a third (101) of the full-text articles came from the journal Molecular Phylogeny and Evolution. Given the subject matter of the journal and the volume of articles its publishes (764 over that 2 year period), this distribution is perhaps to be expected.

\subsection{Results}

See electronic supplementary materials for the full table of evidence and assessment made from each of the 278 papers which the following statistics summarize:

\section{The correlation between citation and usage of the ILD test}

Of the 278 papers manually assessed, I could positively discern that an ILD test was performed and reported in 254 (92\%) of them. In 17 papers (6\%) the ILD test was merely discussed or cited, and explicitly not performed. In 5 papers (2\%) the ILD test was mentioned in the methods section, but no further discussion or report of the result(s) of the test(s) was found - it seems the author(s) in these cases may have simply forgotten to report the results of the ILD tests they refer to in their method sections.

\section{Reporting ILD test $p$-values}

Of the 259 papers that refer to the ILD test as if they had performed one or more tests, 207 $(80 \%)$ reported exact p-values as results of the test(s). 48 papers $(18.5 \%)$ did not report $p$ value result(s) of the ILD test(s) performed, mostly instead reporting either that the ILD test results were "significant" or "not significant". 4 papers provided only information that the pvalue was less than or greater than a number, an inexact numerical answer. 


\section{Reporting ILD test reps}

Replications ('reps') determine in part the robustness of the test applied, thus it is important to report the number of reps.

Of the 259 papers that refer to the ILD test as if they had performed one or more tests, only $131(51 \%)$ reported the number of replications of the ILD test used to determine the null distribution. The number of replications used varied: 10, 100, 200, 500, 1000, 1100, 2000,5000 , or 10000 reps were reportedly used. The majority of papers (77) that reported this parameter used 1000 replications. 14 papers reported using more than 1000 replications, whilst 54 papers reported using less than 1000 replications.

\section{Reporting ILD test search methods}

Of the 259 papers that refer to the ILD test as if they had performed one or more tests, 175 papers $(68 \%)$ clearly specified the search methods used for their ILD tests. The vast majority reported using some form of heuristic random addition sequence + tree bisection reconnection (RAS + TBR) based search using PAUP*, however 5 papers are notable for claiming to have performed their ILD test searches using exhaustive "bandb" searches (Matter 2009; Moyer 2009; Saarma 2009; Meredith 2010; Wei 2010).

\section{Reporting ILD test significance levels used}

Of the 259 papers that refer to the ILD test as if they had performed one or more tests, only 61 papers ( $24 \%$ ) explicitly state the significance threshold (or critical value) by which they judged the significance/non-significance of the ILD test(s) performed. The most commonly reported significance level used was 0.05 , which 35 papers reported. But 26 other papers reported using smaller significance levels in their determination, using either: $0.0001,0.001,0.005$, or 0.01 as their threshold.

\section{Excluding parsimony-uninformative characters}

Of the 259 papers that refer to the ILD test as if they had performed one or more tests, only $32(12 \%)$ papers clearly indicated that parsimony-uninformative characters were removed prior to performing the ILD test. Perhaps a small minority of data sets analysed had no uninformative characters to remove, but this seems an unlikely explanation for all.

\section{"Combinability"}

It was much harder to identify this factor conclusively, but as a conservative estimate, of the 259 papers that refer to the ILD test as if they had performed one or more tests, at 
least 82 (32\%) appeared to have performed the test in order to assess the 'combinability' of their data. As an example of this I quote Bloech et al. (2009):

"The combinability of ITS and matK was tested using the Incongruence Length Difference (ILD) test (Farris et al.., 1994) implemented as partition-homogeneity test in PAUP..."

\subsection{Discussion}

Confusion still reigns over what the ILD test should be used for. Even though clearly inappropriate, a third of papers assessed here appear to mistakenly use the ILD test as an arbiter of combinability; a function for which the test was not designed. Several significant papers published subsequently also clearly indicate that it should not be used as such (Farris 1997; Yoder et al. 2001; Barker \& Lutzoni 2002). Furthermore references to the ILD test as a measure of "phylogenetic accuracy", "topological congruence" or "compatibility" can also be found in the modern usages sampled. It is unclear why this is so.

Regardless of the reasoning behind why the tests are performed, there is also cause for concern with the standard of statistical reporting of the ILD test. As demonstrated, a variety of significance levels are being used. This is defensible given the remarks and suggestions of Cunningham (1997b) about the conservativeness of the test. However, from the standpoint of consistency of interpretation between studies, this is less desirable. For example, Ngamskulrungroj et al. (2009) chose a significance level of 0.0001 , found a Pvalue of 0.002 and therefore reported no significant incongruence (in most other papers this would have been judged significant). Fully $76 \%$ of papers did not explicitly report the significance level they used and $20 \%$ did not report an exact $p$-value which hampers scrutability and re-use of their results. This tallies with similar such studies in other fields e.g. psychology (Bakker \& Wicherts 2011), psychiatry (Berle \& Starcevic 2007) and conservation biology (Fidler et al 2006) that also show disappointing reporting standards and inconsistencies of null-hypothesis significance tests across papers.

It would have been good to go through the thousands of papers that cite the ILD test and perform comparative cladistic analyses of the congruence of typical nuclear and mitochondrial genes found, extending and generalizing upon the initial investigation of 13 different data sets by Fisher-Reid and Wiens (2011). But given how many studies in this sample don't report enough of the vital information (exact p-values, the exclusion of 
invariant characters, the number of replications used, the search method) it appears that the approach of Fisher-Reid and Wiens (2011) (recalculating new ILD tests themselves) is justified.

Although many implementations of the ILD test allow comparisons between three or more data partitions simultaneously, the single p-value result is difficult to interpret in these cases. Giribet (2010) makes this point well as have others. I would recommend always performing pairwise ILD tests between partitions $\left(\left(n^{2}-n\right) / 2\right.$ tests for $n$ partitions $)$ in order to identify the precise source(s) of the incongruence.

Finally, as reported in Allard et al. (1999a), I demonstrated that ILD tests performed on the exactly the same dataset, often appear to give significantly different results between the PAUP* 4.0b10 and TNT (Goloboff et al. 2008) implementations. As both are closed source programs I cannot examine the source code to definitively conclude which is 'wrong' or 'right'.

\subsection{Conclusion}

The ILD test has had a long and tortuous history of development, critique, and usage. Debate has flipped back and forth between positions: useful or useless? Relevant facts and observations about it are scattered across many different papers, some of which are not well cited e.g. (Farris, 1997). Thus as I have demonstrated here the usage of the ILD is sometimes rather inconsistent and confused. In order to help the community better understand the test, and how it should be reported I will conclude with some clear recommendations and clarifications:

- The ILD test (Farris, 1995a) is a measure of the character congruence of data partitions in the context of parsimony analyses.

- It is NOT a measure of, or reliable proxy for; (1) the topological congruence between partitions, (2) phylogenetic accuracy, (3) the "combinability" of partitions, (4) optimising model choice (but see a related derivative of the ILD in Sharma et al. 2011). 
- The ILD test is not appropriately cited as a justification for combining data from different sources prior to analysis (Siddall 1997). Rather, it is sufficient to invoke Kluge's observation that the strongest test of a hypothesis uses the maximum amount of relevant data (Requirement of Total Evidence; Kluge 1989)

- The ILD test is a useful way to investigate the character congruence between data partitions. However, care should be taken not to overinterpret what the result means as there could be a variety of explanations for the level of incongruence found (e.g. heterotachy, noise, 'real' differences in evolutionary signal from hybridization).

- Parsimony-uninformative characters must be excluded before performing the ILD test (Cunningham, 1997b ; Lee 2001).

- Always perform the ILD test with a minimum of 1000 replications to be sure of a robust result, preferably using new technology searches to ensure the minimum length is found.

- Always report the exact $p$-value obtained, the number of replications, the significance level, the software implementation (e.g. PAUP* 4.0b10), and that you excluded invariant characters prior to testing.

- Preferably, perform pairwise tests between each partition as these are easier to interpret than multipartition ILD tests. 


\section{Chapter 5: A modification of Archie's Homoplasy Excess Ratio in the presence of missing data}

\subsection{Abstract}

In this short chapter, I demonstrate a problem with Archie's (1989) Homoplasy Excess Ratio measure, that arises when applied to matrices containing missing or inapplicable data. Sparsely-populated matrices are commonly encountered in 'supermatrix' studies, palaeontological studies, and studies that make heavy use of contingent character coding schemes. I proceed to demonstrate a logical solution to the problem as inspired by Wilkinson's use of selective character permutation, maintaining the position of missing/inapplicable states, in his Phylogenetic Inference by Compatibility Analysis (PICA) program. I implement this idea using TNT and compare this new modified-HER to other measures of homoplasy.

\subsection{Introduction}

The Homoplasy Excess Ratio (HER; Archie, 1989) was introduced as an alternative measure of homoplasy for cladistic data matrices. Existing measures of homoplasy such as the consistency index ( $\mathrm{Cl}$; Kluge \& Farris, 1969) are known to have several significant flaws. Central to the calculation of HER is a randomisation procedure that operates by permuting character states within characters for all characters in the matrix, thereby disrupting the phylogenetic signal in the original unpermuted state of the matrix. The HER is thus insensitive to the inclusion or exclusion of parsimony-uninformative characters from a matrix, unlike the $\mathrm{Cl}$. A large number of such permuted matrices are then analysed under maximum parsimony in order to obtain a distribution of optimal tree lengths corresponding 
to permutations of the matrix. This is similar to the procedure implemented by the permutation tail probability test (PTP; Faith \& Cranston 1991). The limitations of the latter test have been rehearsed at length elsewhere (Wills 1999). However, the HER differs fundamentally from the PTP, because it does not use this distribution as the means to test a null. Rather, the mean value for randomly-permuted matrices (MEANNS) is used as an estimate of the expected tree length for matrices of the same dimensions and with identical frequency distributions of states as the original. The formula for it's calculation is given below:

$$
\mathrm{HER}=(\text { MEANNS }-\mathrm{L}) /(\text { MEANNS }- \text { MINL })
$$

where $L$ is the optimal tree length of the original dataset and MINL is the total number of character states in the entire matrix, minus the number of characters - i.e. the minimum possible tree length to explain all the character transformations in the matrix.

A worked example of the HER calculation using 34 unordered, equally-weighted, parsimony-informative, cranial characters from the pterosaur matrix of Andres et al. (2010) is demonstrated below. Matrix permutations and Maximum Parsimony optimizations for this chapter were all performed in TNT (Goloboff et al 2008) using New Technology searches (Nixon, 1999; Goloboff, 1999):

xperm; xmult=level10; rseed* $^{*} /^{*}$ permute matrix, get tree length, set new seed $*$ The above commands were used to generate 1000 matrix permutation randomisations, the optimal tree lengths of which are plotted in the histogram below. The optimal tree length of the original unpermuted matrix $(\mathrm{L})$ is 59 , whilst the mean optimal tree length of 1000 permuted matrices is 99.419 (MEANNS). The minimum possible tree length for a matrix of these dimensions and state frequencies (MINL) is given in TNT by issuing the command minmax*. In this case MINL is 38 . 


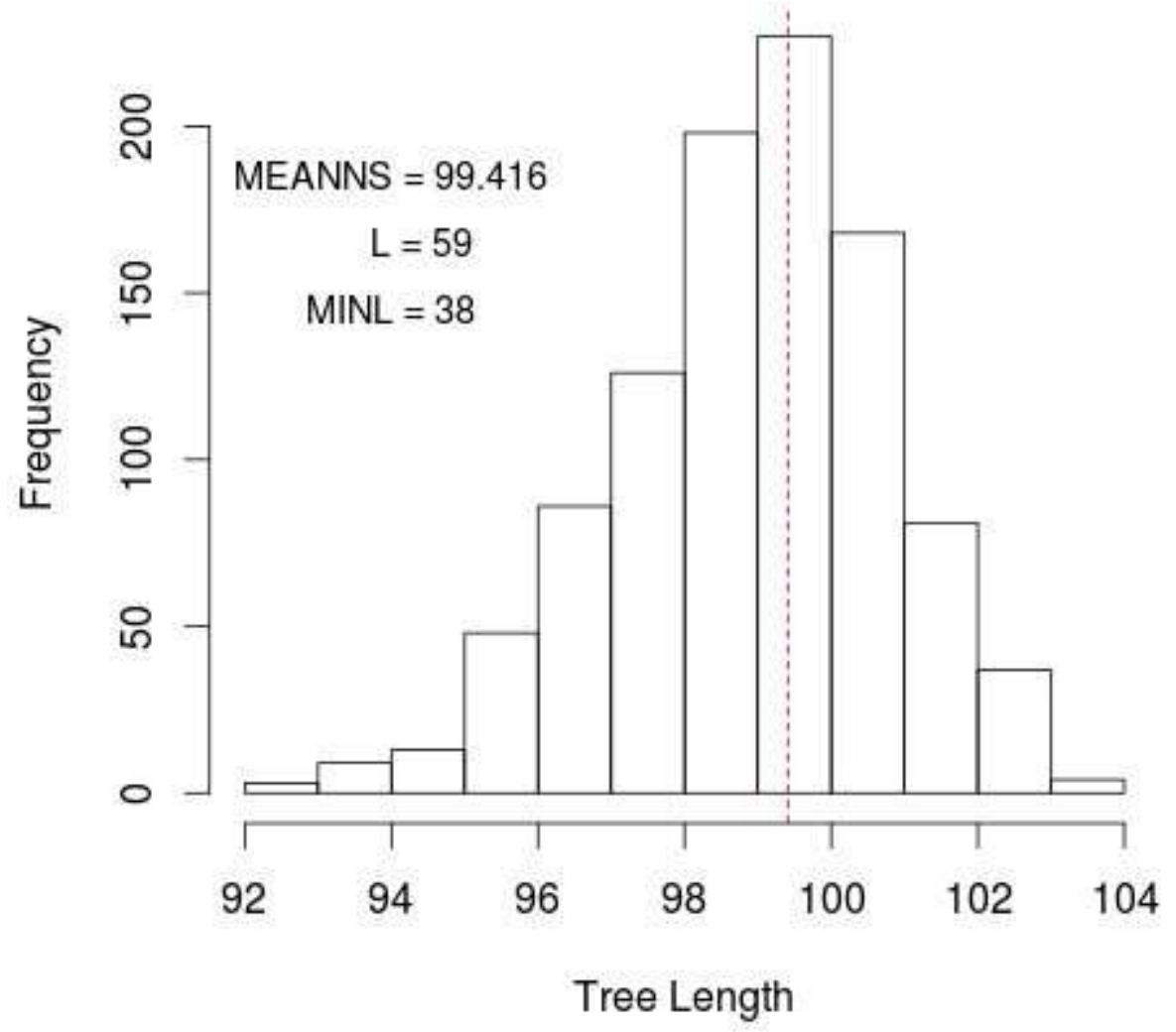

Figure 5.1: The distribution of permuted matrix tree lengths for 1000 permutations of the matrix in appendix 5.1

Thus for the Andres et al. (2010) cranial-only matrix;

HER $=($ MEANNS $-\mathrm{L}) /($ MEANNS - MINL)

HER $=(99.419-59) /(99.419-38)$.

HER $=0.658$

\subsubsection{The Problem of Permuting Missing / Inapplicable Data in Matrices}

During the course of my research I noticed some curious HER results for some matrices that did not match-up to my expectations. There were some very low $<0.1$ values, even some negative values coming from the calculation of HER on sparse matrices. Farris (1991, p85) correctly identified that HER could result in negative values but did not precisely describe the circumstances under which this occurs, nor attempt to provide a solution other than using his RI (Farris, 1989) measure instead. Incidentally, Farris was 
incorrect in saying that "Negative HER might well be called typical of randomizations" (1991, p85) as I demonstrate in Table 5.1; few empirical cladistic matrices generate negative HER values. I demonstrate this undesirable behaviour with HER on an exaggerated sparsely-populated matrix - figure 5.2. This matrix is composed almost entirely of compatible characters except for character 10 which conflicts with characters 9 \& 11 (highlighted in bold). One would therefore expect a relatively low level of homoplasy to be indicated by homoplasy measures.

\begin{tabular}{|c|c|c|c|c|c|c|c|c|c|c|c|c|c|c|c|c|c|c|}
\hline 1 & $\odot$ & $?$ & $?$ & $?$ & $?$ & $?$ & $?$ & $?$ & $?$ & $?$ & $?$ & ? & ? & $?$ & 2 & , & 2 & \\
\hline t2 & 0 & 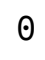 & $?$ & $?$ & $?$ & $?$ & $?$ & $?$ & $?$ & $?$ & $?$ & ? & ? & ? & $?$ & & & 1 \\
\hline t3 & $?$ & 0 & 0 & $?$ & $?$ & $?$ & $?$ & $?$ & $?$ & $?$ & $?$ & $?$ & • & • & $\cdot$ & & & $\perp$ \\
\hline$=4$ & $?$ & $?$ & 0 & $\odot$ & $?$ & $?$ & $?$ & $?$ & $?$ & $?$ & $?$ & $?$ & ? & & & & & \\
\hline 5 & $?$ & $?$ & $?$ & $\odot$ & $\odot$ & $?$ & $?$ & $?$ & $?$ & $?$ & $?$ & ? & ? & & & & & 2 \\
\hline$=6$ & $?$ & $?$ & $?$ & $?$ & 0 & 0 & $?$ & $?$ & $?$ & $?$ & $?$ & ? & ? & & & & & \\
\hline$=7$ & $?$ & $?$ & $?$ & $?$ & $?$ & 0 & 0 & $?$ & $?$ & $?$ & $?$ & ? & 1 & & $?$ & & & \\
\hline$=8$ & $?$ & $?$ & $?$ & $?$ & $?$ & $?$ & $\theta$ & $\odot$ & $?$ & $?$ & $?$ & 1 & 1 & ? & ? & & & \\
\hline t9 & $?$ & $?$ & $?$ & $?$ & $?$ & $?$ & $?$ & 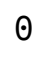 & 0 & 1 & 1 & 1 & ? & & & & ? & ? \\
\hline t 10 & $?$ & $?$ & $?$ & $?$ & $?$ & $?$ & $?$ & $?$ & 0 & 0 & 1 & ? & $?$ & ? & ? & $?$ & $?$ & ? \\
\hline t 11 & $?$ & $?$ & $?$ & $?$ & $?$ & $?$ & $?$ & $?$ & 1 & 1 & 0 & $?$ & 2 & 2 & 2 & 7 & 7 & $?$ \\
\hline 12 & $?$ & $?$ & $?$ & $?$ & $?$ & $?$ & $?$ & 1 & 1 & 0 & 0 & 0 & $?$ & & $\cdot$ & & 2 & 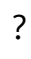 \\
\hline 13 & $?$ & $?$ & $?$ & $?$ & $?$ & $?$ & 1 & 1 & $?$ & $?$ & $?$ & 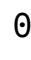 & $v$ & • & 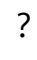 & & : & $?$ \\
\hline 14 & $?$ & $?$ & $?$ & $?$ & $?$ & 1 & 1 & $?$ & $?$ & $?$ & $?$ & $?$ & 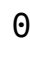 & 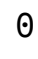 & : & 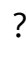 & $?$ & $?$ \\
\hline t15 & $?$ & $?$ & $?$ & $?$ & 1 & 1 & $?$ & $?$ & $?$ & $?$ & $?$ & ? & ? & $\odot$ & $\odot$ & $?$ & ? & ? \\
\hline t 16 & $?$ & $?$ & $?$ & 1 & 1 & $?$ & $?$ & $?$ & $?$ & $?$ & $?$ & ? & ? & ? & 0 & $\theta$ & ? & $?$ \\
\hline t 17 & $?$ & $?$ & 1 & 1 & $?$ & $?$ & $?$ & $?$ & $?$ & $?$ & $?$ & ? & $?$ & ? & $?$ & 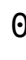 & $\odot$ & ? \\
\hline t18 & 2 & 1 & 1 & $?$ & $?$ & $?$ & $?$ & $?$ & $?$ & $?$ & $?$ & ? & ${ }^{\circ}$ & & 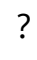 & ${ }^{\circ}$ & $\theta$ & $\odot$ \\
\hline 19 & 1 & 1 & $?$ & $?$ & $?$ & $?$ & $?$ & $?$ & $?$ & $?$ & $?$ & ? & ? & 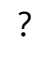 & 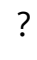 & 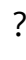 & ? & $\odot$ \\
\hline 20 & 1 & $?$ & $?$ & $?$ & $?$ & $?$ & $?$ & $?$ & $?$ & $?$ & $?$ & ? & ? & ? & ? & ? & ? & ? \\
\hline
\end{tabular}

Figure 5.2: A hypothetical sparse matrix of 19 parsimony-informative characters and 20 taxa.

Indeed the Consistency Index $(\mathrm{Cl})$ for this matrix is 0.950 . But the HER is -24 .

The problem with permuting the entirety of character columns, is that with sparsely-scored matrices such as in figure 5.2, character permutations are likely to artefactually decrease the probability of character conflict, resulting in lowered MEANNS values. Figure 5.3 demonstrates one such permutation whereby the missing states get permuted in such a way that taxon 5 to taxon 20 become completely missing, and two pairs of taxa complete 
with data, become identical (taxon 1 is identical in state composition to taxon 2; likewise for taxon 3 and taxon 4).

The HER calculation for this sparse matrix is: HER $=(19.04-20) /(19.04-19)$, HER $=$ -24 .

$\begin{array}{lllllllllllllllllllll}\mathrm{t} 1 & 0 & 0 & 0 & 0 & 0 & 0 & 0 & 0 & 0 & 0 & 0 & 0 & 0 & 0 & 0 & 0 & 0 & 0 & 0 \\ \mathrm{t} 2 & 0 & 0 & 0 & 0 & 0 & 0 & 0 & 0 & 0 & 0 & 0 & 0 & 0 & 0 & 0 & 0 & 0 & 0 & 0 \\ \mathrm{t} 3 & 1 & 1 & 1 & 1 & 1 & 1 & 1 & 1 & 1 & 1 & 1 & 1 & 1 & 1 & 1 & 1 & 1 & 1 & 1 \\ \mathrm{t} 4 & 1 & 1 & 1 & 1 & 1 & 1 & 1 & 1 & 1 & 1 & 1 & 1 & 1 & 1 & 1 & 1 & 1 & 1 & 1 \\ \mathrm{t} 5 & ? & ? & ? & ? & ? & ? & ? & ? & ? & ? & ? & ? & ? & ? & ? & ? & ? & ? & ? \\ \mathrm{t} 6 & ? & ? & ? & ? & ? & ? & ? & ? & ? & ? & ? & ? & ? & ? & ? & ? & ? & ? & ? \\ \mathrm{t} 7 & ? & ? & ? & ? & ? & ? & ? & ? & ? & ? & ? & ? & ? & ? & ? & ? & ? & ? & ? \\ \mathrm{t} 8 & ? & ? & ? & ? & ? & ? & ? & ? & ? & ? & ? & ? & ? & ? & ? & ? & ? & ? & ? \\ \mathrm{t} 9 & ? & ? & ? & ? & ? & ? & ? & ? & ? & ? & ? & ? & ? & ? & ? & ? & ? & ? & ? \\ \mathrm{t} 10 & ? & ? & ? & ? & ? & ? & ? & ? & ? & ? & ? & ? & ? & ? & ? & ? & ? & ? & ? \\ \mathrm{t} 11 & ? & ? & ? & ? & ? & ? & ? & ? & ? & ? & ? & ? & ? & ? & ? & ? & ? & ? & ? \\ \mathrm{t} 12 & ? & ? & ? & ? & ? & ? & ? & ? & ? & ? & ? & ? & ? & ? & ? & ? & ? & ? & ? \\ \mathrm{t} 13 & ? & ? & ? & ? & ? & ? & ? & ? & ? & ? & ? & ? & ? & ? & ? & ? & ? & ? & ? \\ \mathrm{t} 14 & ? & ? & ? & ? & ? & ? & ? & ? & ? & ? & ? & ? & ? & ? & ? & ? & ? & ? & ? \\ \mathrm{t} 15 & ? & ? & ? & ? & ? & ? & ? & ? & ? & ? & ? & ? & ? & ? & ? & ? & ? & ? & ? \\ \mathrm{t} 16 & ? & ? & ? & ? & ? & ? & ? & ? & ? & ? & ? & ? & ? & ? & ? & ? & ? & ? & ? \\ \mathrm{t} 17 & ? & ? & ? & ? & ? & ? & ? & ? & ? & ? & ? & ? & ? & ? & ? & ? & ? & ? & ? \\ \mathrm{t} 18 & ? & ? & ? & ? & ? & ? & ? & ? & ? & ? & ? & ? & ? & ? & ? & ? & ? & ? & ? \\ \mathrm{t} 19 & ? & ? & ? & ? & ? & ? & ? & ? & ? & ? & ? & ? & ? & ? & ? & ? & ? & ? & ? \\ \mathrm{t} 20 & ? & ? & ? & ? & ? & ? & ? & ? & ? & ? & ? & ? & ? & ? & ? & ? & ? & ? & ?\end{array}$

Figure 5.3: A possible permutation of the sparse matrix from figure 5.2

Thus I have identified that HER suffers from a missing data problem which has not been noticed until now. The HER aims to be a measure of the 'global' homoplasy across the known states of characters in a cladistic matrix. Yet clearly, the calculation is confounded by the introduction of many unknown states (missing data). A desirable measure of global homoplasy should measure purely the homoplasy of known character states, unaffected by the presence, quantity or distribution of missing data around the known data. Thus I propose here a novel modification to the calculation of HER that estimates MEANNS based-only upon the subset of all possible permutations that keep the unknown states 
fixed in the same position as they are in the original unpermuted matrix.

This modified-HER (MHER) reflects the level of homoplasy only in the known states of characters, unconfounded by missing data issues, or matrix parameter issues (e.g. the problems of Cl; Sanderson and Donoghue 1989; Meier et al. 1991; Klassen et al 1991).

\subsubsection{Time-Efficient Computational Implementation}

Drawing inspiration from the implementation of selective-permutations in Wilkinson's PICA program for compatibility analyses, I reasoned that character column permutations restricted to permuting only information-containing states would help maintain the structure of informative-states in taxa, whilst still allowing permutations within that structure. In this new scheme of permutation, permutations such as that depicted in figure 6.3 would not be allowed. With the help of Pablo Goloboff I have implemented the character permutation of solely information-containing states in TNT (Goloboff et al. 2008). The script is given in Appendix 6.2. This enables MHER to be calculated for most morphology-based data sets with 1000 replications in a reasonable time (e.g. less than 2 hours to get MHER for Asher et al's (2005) matrix of 223 characters and 68 taxa running on a simple Phenom X3 2.3Ghz desktop computer). Small data sets take just seconds to process.

To make it easier to use this selective-permutation script, I have additionally written a bash script which when passed the name of a dataset in .tnt format, launches TNT, calls the script in appendix 6.2, calculates and reports L, MINL, and the modified-MEANNS value, as well as calculating and reporting the final MHER using awk for the final arithmetic operation (appendix 6.3).

The MHER of the sparse matrix in 6.2 is now calculated to give a more intuitive result:

MHER $=(25.4815-20) /(25.4815-19)=0.845715$

Of the informative data states that are in the data matrix, this relatively high value of MHER demonstrates that per character there is relatively little homoplasy in the matrix relative to the optimal cladogram. 


\subsubsection{How many replications are needed for a robust MEANNS estimate?}

Archie (1996, p162) states that "in most cases... with good precision... no more than 25 " matrix permutation reps are needed to calculate MEANNS, without supporting evidence. Whilst I agree that there is relatively little variance, I observed it to be non-negligible. Thus here, I quantify the variance using the Carrano \& Sampson (2008) dataset. Ten-thousand matrix permutations and their optimal lengths were recorded. The mean optimal length of these ten-thousand permutations was 365.57 (to 2 d.p.), the maximum value was 375 , the minimum value was 353 and the standard deviation was 2.96 .

Are parametric statistics really justified in this case? Most matrix permutation tree length distributions are significantly non-Normal according to tests such as Anderson-Darling's (data not shown, see also figure 5.1 for a graphical example). But with large sample sizes such tests of normality are often over-powered and give a 'significant' result even if deviation from normality is relatively small, thus I will continue to treat these tree lengths as if they were normally distributed.

\subsubsection{Is the HER missing data problem significant in real matrices?}

It is all fine and well to demonstrate a problem that could happen in theory, but does the HER suffer missing data effects in practice? To assess the difference between HER, MHER and $\mathrm{Cl}$, on real empirical matrices from the published literature I tested 60 matrices. Some were garnered directly from the literature (PDFs) by myself, whilst others were gratefully obtained from Graeme Lloyd's excellent shared collection of matrices (http://www.graemetlloyd.com/matr.html), from MorphoBank (O'Leary \& Kaufman 2011), or from TreeBASE (Piel et al. 2002). The results of these analyses are below in Table 5.1. Most were conducted with 1000 matrix permutation reps, except the 10 molecular supermatrices, which I calculated using only 100 matrix permutations. Admittedly parsimony may not be the best method for inferring phylogeny from molecular sequences but I have included these 10 matrices nonetheless to demonstrate the effect of very sparse matrices upon HER.

\subsection{Method}

All data sets were analysed in TNT (Goloboff et al. 2008) using New Technology searches 
(Goloboff, 1999; Nixon 1999) xmult=level10 + bb as well as the scripts provided in the appendices for chapter 5 .

\subsection{Results}

Table 5.1 An empirical comparison of measures of homoplasy, sorted by missing data, for 60 assorted data sets including 7 molecular data supermatrices which are indicated with an asterix*

\begin{tabular}{|c|c|c|c|c|c|c|c|c|}
\hline 1stAuthor Year & Group & \#Chars & \#Taxa & \%Miss. & HER & MHER & CI & mean ci \\
\hline Thomson 2010 & Testudines* & 3406 & 213 & 98.4 & $-\mathrm{INF}$ & 0.787 & 0.994 & 1.000 \\
\hline Csiki 2010 & Theropoda & 364 & 36 & 87.6 & 0.231 & 0.248 & 0.423 & 0.542 \\
\hline Kurochkin 1996 & Enantiornithes & 122 & 40 & 80.7 & 0.295 & 0.421 & 0.692 & 0.790 \\
\hline Hinchliff 2013 & Cyperaceae* & 16016 & 435 & 78.7 & 0.778 & 0.801 & 0.414 & 0.859 \\
\hline Wolsan 2010 & Carnivora* & 9753 & 52 & 76.6 & 0.207 & 0.229 & 0.517 & 0.855 \\
\hline VderLinde 2010 & Drosophilidae* & 14912 & 180 & 73.6 & 0.429 & 0.240 & 0.289 & 0.811 \\
\hline Springer 2012 & Primates* & 61199 & 372 & 68.6 & 0.775 & 0.832 & 0.358 & 0.863 \\
\hline Pirie 2008 & Danthonioid grasses* & 11810 & 298 & 68.1 & 0.846 & 0.878 & 0.577 & 0.940 \\
\hline Moore 2011 & Angiosperms* & 69513 & 246 & 64.6 & 0.879 & 0.899 & 0.214 & 0.584 \\
\hline Brusatte 2012 & Theropoda & 233 & 46 & 57.6 & 0.448 & 0.460 & 0.441 & 0.566 \\
\hline McDonald 2010 & Iguanodontia & 135 & 67 & 57.4 & 0.662 & 0.674 & 0.489 & 0.625 \\
\hline Carballido 2010 & Sauropoda & 104 & 19 & 51.7 & 0.642 & 0.659 & 0.703 & 0.820 \\
\hline Carrano 2008 & Ceratosauria & 151 & 18 & 48.1 & 0.712 & 0.724 & 0.732 & 0.829 \\
\hline Friedman 2007 & Actinistia & 195 & 39 & 44.6 & 0.567 & 0.573 & 0.456 & 0.587 \\
\hline $\begin{array}{l}\text { Gonzalez-Riga } \\
2009\end{array}$ & Titanosauria & 102 & 23 & 42 & 0.478 & 0.484 & 0.617 & 0.751 \\
\hline Young 2009 & Crocodylomorpha & 166 & 86 & 40.7 & 0.792 & 0.793 & 0.447 & 0.646 \\
\hline Butler 2008 & Ornithischia & 221 & 46 & 39.9 & 0.619 & 0.624 & 0.505 & 0.649 \\
\hline Gaffney 2009 & Bothremydidae & 175 & 47 & 37.2 & 0.715 & 0.718 & 0.581 & 0.732 \\
\hline Skutchas 2012 & Caudata & 72 & 21 & 35.3 & 0.261 & 0.271 & 0.497 & 0.641 \\
\hline Sereno 2008 & Carcharodontosaurids & 60 & 9 & 30.9 & 0.559 & 0.565 & 0.738 & 0.822 \\
\hline Bloch 2007 & Plesiadapiforms & 173 & 21 & 29.6 & 0.338 & 0.341 & 0.448 & 0.539 \\
\hline Martinez 2009 & Ictidosauria & 98 & 12 & 29.4 & 0.317 & 0.326 & 0.561 & 0.683 \\
\hline Hospitaleche 2007 & Sphenisciformes & 70 & 29 & 27 & 0.521 & 0.524 & 0.474 & 0.598 \\
\hline Simmons 2008 & Mormoopidae & 202 & 29 & 27 & 0.463 & 0.465 & 0.408 & 0.518 \\
\hline Frobisch 2007 & Dicynodontia & 100 & 42 & 26.8 & 0.649 & 0.651 & 0.470 & 0.598 \\
\hline Lu 2009 & Pterosauria & 52 & 16 & 25.4 & 0.496 & 0.500 & 0.562 & 0.685 \\
\hline Anderson 2008 & Batrachia & 216 & 54 & 23.4 & 0.561 & 0.418 & 0.248 & 0.348 \\
\hline Ezcurra 2007 & Coelophysoidea & 136 & 13 & 22.7 & 0.591 & 0.593 & 0.633 & 0.714 \\
\hline Matsumoto 2009 & Dinosauria & 76 & 16 & 22 & 0.517 & 0.520 & 0.572 & 0.644 \\
\hline Asher 2005 & Lagomorpha & 223 & 68 & 18.2 & 0.467 & 0.562 & 0.239 & 0.326 \\
\hline
\end{tabular}




\begin{tabular}{|c|c|c|c|c|c|c|c|c|}
\hline Holland 2009 & Tetrapodomorpha & 44 & 22 & 17.8 & 0.429 & 0.435 & 0.441 & 0.557 \\
\hline Asher 2006 & Afrotheria & 112 & 23 & 17.2 & 0.418 & 0.421 & 0.405 & 0.470 \\
\hline Mueller 2006 & Choristodera & 90 & 25 & 16.8 & 0.457 & 0.459 & 0.415 & 0.569 \\
\hline Weksler 2006 & Oryzomyini & 99 & 54 & 12.6 & 0.414 & 0.414 & 0.285 & 0.439 \\
\hline $\begin{array}{l}\text { Sanchez-Villagra } \\
2006\end{array}$ & Talpidae & 157 & 21 & 12.4 & 0.528 & 0.529 & 0.452 & 0.568 \\
\hline Shimada 2005 & Lamniformes & 55 & 17 & 12.4 & 0.551 & 0.552 & 0.525 & 0.640 \\
\hline Cheng 2012 & Eosauropterygia & 139 & 35 & 12.4 & 0.548 & 0.549 & 0.389 & 0.491 \\
\hline Smith 2011 & Alcidae & 223 & 59 & 12.4 & 0.584 & 0.586 & 0.211 & 0.377 \\
\hline Friedman 2008 & Pleuronectiformes & 58 & 19 & 11.8 & 0.582 & 0.582 & 0.507 & 0.596 \\
\hline Asher 2007 & Eutheria & 190 & 53 & 10.3 & 0.338 & 0.338 & 0.227 & 0.359 \\
\hline Wiens 2005 & Hylidae & 140 & 81 & 8.3 & 0.373 & 0.374 & 0.184 & 0.269 \\
\hline Mayr 2011b & Pelagornithidae & 87 & 25 & 8.3 & 0.447 & 0.449 & 0.377 & 0.499 \\
\hline Pine 2012 & Cricetidae & 89 & 36 & 8.3 & 0.256 & 0.257 & 0.279 & 0.378 \\
\hline Worthy 2009 & Anseriformes & 150 & 62 & 8.1 & 0.429 & 0.429 & 0.201 & 0.297 \\
\hline Whitlock 2011 & Diplodocidae & 189 & 27 & 7.6 & 0.759 & 0.767 & 0.739 & 0.472 \\
\hline Bourdon 2009 & Palaeognathae & 129 & 17 & 6.9 & 0.936 & 0.936 & 0.876 & 0.922 \\
\hline Venczel 2008 & Caudata & 35 & 15 & 6.7 & 0.665 & 0.666 & 0.629 & 0.714 \\
\hline Hill 2005 & Amniota & 48 & 18 & 6.5 & 0.674 & 0.674 & 0.634 & 0.750 \\
\hline Jouve 2006 & Crocodylomorpha & 116 & 64 & 5.3 & 0.666 & 0.667 & 0.335 & 0.560 \\
\hline Parenti 2008 & Ankylosauria & 80 & 31 & 5 & 0.726 & 0.725 & 0.595 & 0.736 \\
\hline Mauricio 2012 & Rhynocriptidae & 90 & 38 & 3.8 & 0.722 & 0.722 & 0.485 & 0.664 \\
\hline Mayr 2010a & $\begin{array}{l}\text { Quercypsitta-Like } \\
\text { Birds }\end{array}$ & 93 & 32 & 3.7 & 0.371 & 0.372 & 0.343 & 0.484 \\
\hline Gaubert 2005 & Pholidota & 329 & 44 & 3.4 & 0.521 & 0.521 & 0.323 & 0.412 \\
\hline Smith 2010 & Pelecaniformes & 464 & 53 & 3.3 & 0.804 & 0.804 & 0.445 & 0.594 \\
\hline Sparks 2008 & Etroplinae & 80 & 25 & 3 & 0.912 & 0.912 & 0.737 & 0.853 \\
\hline Mayr 2010b & Caprimulgiform Birds & 69 & 10 & 2.3 & 0.570 & 0.570 & 0.649 & 0.762 \\
\hline DePietri 2011 & Lari & 40 & 12 & 1.7 & 0.459 & 0.460 & 0.695 & 0.800 \\
\hline Li 2007 & Squamata & 62 & 10 & 1.3 & 0.317 & 0.317 & 0.647 & 0.701 \\
\hline Manegold 2013 & Picidae & 67 & 27 & 0.8 & 0.803 & 0.803 & 0.490 & 0.696 \\
\hline Hurley 2007 & Actinopterygii & 31 & 8 & 0 & 0.425 & 0.425 & 0.653 & 0.737 \\
\hline
\end{tabular}

Assuming normality, using the function power . $t$. test in $\mathrm{R}(\mathrm{R}$ Development Core Team, 2013), it can be shown that at the $1 \%$ significance level, for a two-tailed comparison between normal matrix permutation MEANNS (HER) and selective matrix permutation MEANNS (MHER), with 1000 replications each, a significant difference (delta) in sample mean of just 1 step in tree length can be detected with power $>0.99$. 
(Two-sample t test power calculation: $n=1000$, delta $=1$, sd $=2.96$, sig.level $=0.01$, alternative $=$ two. sided $)$.

A difference of one step in mean tree length between the two types of permutations for 1000 reps in the case of the Carrano \& Sampson (2008) dataset translates into a difference of approximately 0.0013 HER. Thus when comparing between HER \& MHER results, even for those results that differ in ratio by only 0.001 , this difference is statistically significant. Over half of the data sets tested in Table 5.1 thus display a statistically significant difference between their HER \& MHER values.

\subsection{Discussion}

MHER is a useful measure of the average ('global') homoplasy of a cladistic matrix, comparable between data sets of varying taxa, characters, and percentage completeness. Some claim that such 'global' measures are too crude to be of use; Phillippe et al. (1996) once asserted that "A good measure of homoplasy must look at the characters locally and not globally... we suggest that homoplasy should be measured locally" ( $p$ 1184). I would have to disagree with this. In the context of comparative cladistic analyses, controlled, standardised 'global' measures such as MHER provide useful information - measures of homoplasy that are local to a particular region of a cladogram cannot be compared between studies that do not contain those same taxa. Depending on the hypothesis to be tested either 'global' or 'local' or both approaches may be of utility.

Meier et al. (1991, p77) illustrated an apparent negative correlation between HER and the number of taxa in the matrix based upon an analysis of 27 different matrices. I do not dispute this observation but instead question if matrix parameters are the true cause of the correlation they observed. It is not inconceivable that the larger data sets they tested also contained more missing data, with an uneven distribution of it in the matrices. In my analyses (Table 5.1), excluding the supermatrices $(n=53)$, there is a small positive correlation ( $r=+0.028$ ) between HER \& \#Taxa but it is not significant $(p=0.852)$, and is perhaps better explained by the percentage and distribution of missing/inapplicable data within matrices. In support of this, Archie (1996, p163) also asserts that HER is not affected by matrix parameters (but no evidence is given). 


\subsubsection{Why has HER seemingly been ignored for so long?}

I identified just 75 papers that mention "Homoplasy Excess Ratio" in a Google Scholar search as of 2013-08-01. Many only mention it in passing. One of these hits was my own conference abstract (Mounce, 2011a). I tentatively suggest three factors that might have led to HER being relatively ignored as a measure of homoplasy since it was devised:

1. The computational complexity of the randomisations might have made it relatively unappealing to calculate, relative to other computationally simpler measures of homoplasy. Goloboff (1991) wrote that HER was "difficult to calculate" and Meier et al. (1991) said it was "computationally very expensive and for large analyses impractical". Hence, undue focus was perhaps shifted to its inferior approximations; REHER and HERM (Archie, 1996). Likewise, Fu \& Murphy (1999) later wrote that the "substantial computing time" required was "a major limit of the application of HER" back then.

2. Farris's (1991) paper strongly criticizes HER and argues throughout that RI could and should be used instead. Similarly Meier et al. (1991) observed an apparent negative correlation of HER with matrix parameters. Perhaps these papers deterred people?

3. Confusion between HER and HERM. As HERM was correctly shown to be identical to Farris' RI (Farris 1989; 1991) it is possible that some did not see the distinction between HERM \& HER and thus discounted both when HERM was shown to be redundant. A putative example of this is given by Egan (2006) who mentioned only HERM in her discussion of "goodness of fit metrics".

A new generation of academics are once again investigating important questions about homoplasy (e.g. Hoyal Cuthill et al.. 2010). I have no doubt that there are many further important studies to be done in this area. In future work, I will compare MHER with Goloboff's Data Decisiveness (1991) \& Wilkinson's (1997) incompatibility excess ratio's two other under-utilised descriptive statistics for cladistics. 


\section{Chapter 6: Optimal search strategies for finding phylogenetic data}

\subsection{Abstract}

In the preceding chapters, I have repurposed data from published studies in order to perform comparative cladistic analyses and thereby to test hypotheses that single cladistic dataset studies alone could not adequately address. In order to re-use these data, I had to find relevant studies and transform the published data back into a digitally-usable form neither of which are trivial tasks. In this chapter I explore and critically compare methods of digital literature search in the context of the task of finding morphology-based phylogenetic analyses. Traditional title-keyword-abstract searches with Web of Knowledge \& Scopus, and modern full-text searches as provided by publishers like PLOS \& BMC, as well as offline local desktop full-text searching are compared and discussed. I demonstrate that the popular traditional methods are significantly poorer at finding phylogenies (in terms of recall to known relevant papers) when compared to full-text search methods. I conclude that despite three and half years of looking for morphology-based phylogenetic analyses, I can only put a conservative minimum-bound estimate on the number of morphology-based phylogenetic studies that have been published in the $21^{\text {st }}$ century because of inadequate bulk full-text access to journal literature. 


\subsection{Introduction}

Forty years ago a thorough literature search necessitated a trip to a library so that researchers could systematically examine all relevant journals and books page-by-page to scan for the desired concepts and items of interest. More recently, the ubiquitous electronic publication of research on the Internet has enabled less-manual, more computationally expedited methods of literature search using computer software to scan articles and books for relevant terms and concepts in text-form. To help academics find relevant content (and to make a profit by charging for this commercial service) Thomson Reuters released the first version of Web of Knowledge (WoK) a 'research platform' for academic content discovery over a decade ago - it launched in 2002 (Thomson Reuters, 2013). Shortly afterwards, Elsevier launched a rival profit-making commercial service called Scopus (Fingerman, 2004). Both of these abstract \& citation indexing services are now widely used by researchers in non-biomedical biological sciences. One of them; WoK only indexes the title, abstract, keywords and citations for each article or book chapter.

It is important to note here that I will not discuss PubMedCentral (PMC) as commonly used by biomedical researchers because on the whole it only indexes biomedical content whilst morphology-based phylogenetic content can occasionally be found indexed in PMC, particularly if it appears in a 'general' journal like Nature, Science or PLOS ONE e.g. Eddy \& Clarke (2011), most non-biomedical subject-specific journals e.g. Zootaxa, Palaeontology, and Journal of Vertebrate Paleontology ... are not indexed in PMC. Thus PMC cannot be relied-upon for literature searches for non-biomedically relevant topics. As a further demonstration of this, if one searches for 'Zootaxa' in PMC, one can only find four articles from the journal Zootaxa in PMC that have been self-deposited by their authors as 'author manuscripts' (context: Zootaxa has published over 12,500 articles as of 2013-0701).

Other relevant digital search services that academics sometimes use include Google Scholar (GS; http://scholar.google.com/), Scirus (http://www.scirus.com/), Mendeley Search (MS; http://www.mendeley.com/research-papers/search/) and Microsoft Academic Search beta (MAS; http://academic.research.microsoft.com/). GS first launched nearly ten 
years ago as beta in 2004 (Google, 2013). GS can notably achieve 100\% recall for some searches (Gehanno et al. 2013) and is thus often better than Scopus \& WoK's recall (e.g. Beckmann \& von Wehrden 2012). But the precision of GS is often very poor (Garcia-Perez 2012), since it searches across a much wider body of grey literature: including some blogs, newsletters and non-peer reviewed material It also offers relatively few features with which to constrain or filter searches (other than simple 'by year/journal/author'). Moreover, there is no easy mechanism provided by which hundreds of search results can be exported in a standard format (e.g. bibtex). Thus some have pointed out before that GS is not useful for performing systematic literature searches (Giustini, 2013). Scirus (also known as Sciverse Hub: they are different interfaces to the same index [California Digital Library, 2013]) allows full text search of a limited subset of the research literature, as well as abstract-only search, and grey literature 'scientific web' searches.

For the purposes of this chapter, when referring to Scirus I shall only be referring to the full text search subset of the capabilities of Scirus. MS is a relatively new academic search provider and claims to search across a crowd-sourced database of nearly 100 million documents (Mendeley, 2013). MAS is yet another new academic search provider and is still in active development, the service is self-described on their About page (Microsoft, 2013). GS, Scirus and an earlier version of Microsoft Academic Search have previously been compared (Ford \& O'Hara, 2008) for searches in 2006 during which GS retrieved the most citations, however the aim and methodology of that study is different to the one presented herein, and I anticipate that all of the databases have improved in performance since 2006.

\subsubsection{Preliminary Investigation}

Table 6.1 illustrates some of the complexity of digital literature searches. GS finds the most content overall for a simple keyword search over a defined period (2000-2012) but this includes many books and non-peer reviewed grey literature pieces that one would perhaps want to exclude (with no easy way provided to filter these out). GS also notably does not handle wildcards, so I could not perform a more conservative search for phylogeny-related articles with 'phylog*' to catch the words 'phylogeography', 'phylogram', 'phylogenies' etc... For general searches, in agreement with the findings of Chadegani et al. (2013), Scopus appeared to find more content than WoK but in specific cases (e.g. the searches for Winclada:Table 6.1) WoK occasionally appears to outperform Scopus in raw hits. 
Table 6.1 A comparison of simple search results for the term "phylogeny" and "winclada" in scholarly documents published between the years 2000 - 2012 inclusive, all searches performed 2013-07-15.

\begin{tabular}{|c|c|c|c|}
\hline $\begin{array}{l}\text { Search } \\
\text { Service }\end{array}$ & Term & Exact Repeatable Search Terms Used & Hits \\
\hline $\begin{array}{l}\text { Google } \\
\text { Scholar }\end{array}$ & phylogeny & 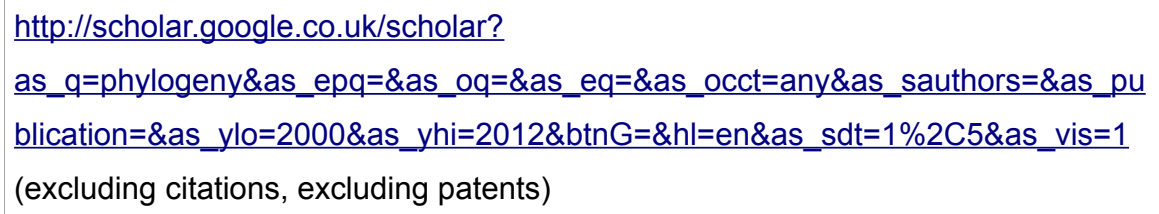 & $\sim 220,000$ \\
\hline Scirus & phylogeny & 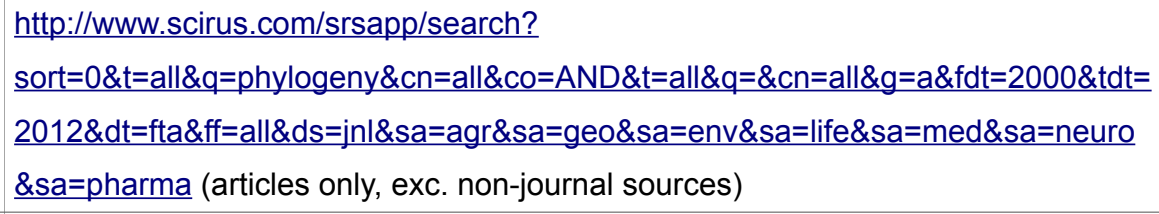 & 43,836 \\
\hline Scirus & phylog* & 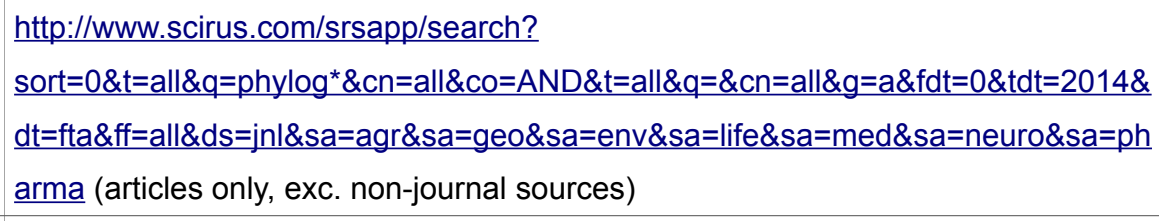 & 163,015 \\
\hline WoK & phylogeny & Topic $=($ phylogeny $)$ Timespan=2000-2012. Databases=SCI-EXPANDED & 44,946 \\
\hline WoK & phylog* & Topic $=($ phylog $*$ Timespan=2000-2012. Databases $=$ SCI-EXPANDED & 120,078 \\
\hline Scopus & phylogeny & $\begin{array}{l}\text { TITLE-ABS-KEY(phylogeny) AND SUBJAREA(mult OR agri OR bioc OR immu } \\
\text { OR neur OR phar OR mult OR medi OR nurs OR vete OR dent OR heal) AND } \\
\text { PUBYEAR > } 1999 \text { AND PUBYEAR }<2013\end{array}$ & 127,991 \\
\hline Scopus & phylog* & $\begin{array}{l}\text { TITLE-ABS-KEY(phylog*) AND SUBJAREA(mult OR agri OR bioc OR immu OR } \\
\text { neur OR phar OR mult OR medi OR nurs OR vete OR dent OR heal) AND } \\
\text { PUBYEAR > } 1999 \text { AND PUBYEAR < } 2013\end{array}$ & 156,583 \\
\hline $\begin{array}{l}\text { Google } \\
\text { Scholar }\end{array}$ & winclada & $\begin{array}{l}\text { http://scholar.google.co.uk/scholar? } \\
\text { q=winclada\&hl=en\&as_sdt=1\%2C5\&as_vis=1\&as_ylo=2000\&as_yhi=2012 } \\
\text { (excluding citations, excluding patents) }\end{array}$ & $\sim 1,840$ \\
\hline Scirus & winclada & 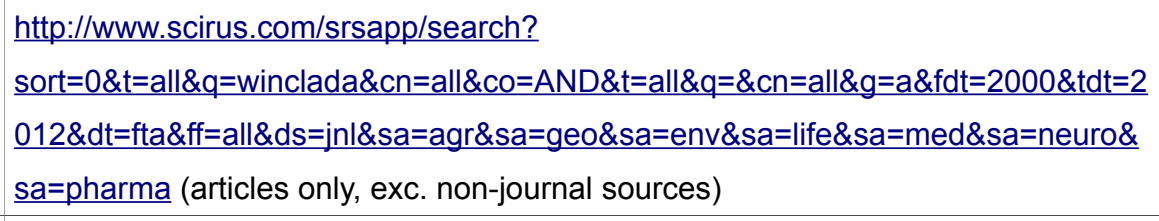 & 292 \\
\hline WoK & winclada & Topic $=($ winclada $)$ Timespan=2000-2012. Databases $=$ SCI-EXPANDED & 18 \\
\hline Scopus & winclada & $\begin{array}{l}\text { TITLE-ABS-KEY(winclada) AND SUBJAREA(mult OR agri OR bioc OR } \\
\text { immu OR neur OR phar OR mult OR medi OR nurs OR vete OR dent OR } \\
\text { heal) AND PUBYEAR > } 1999 \text { AND PUBYEAR < } 2013\end{array}$ & 12 \\
\hline
\end{tabular}

However, relative to GS's fulltext search capability, WoK and Scopus have a markedly diminished ability to find methodological details that are not usually discussed in abstracts. This is why I am doing this chapter - I have noticed during my research that it is very difficult to accurately search for certain types of phylogenetic methods detail. I will use this 
chapter to objectively test the performance of very search methods for finding papers with phylogenetic analyses in them.

It is known that Scopus and WoK have non-overlapping journal coverage (e.g. see Figure 1 of Chadegani et al. 2013). However, even in cases where journal coverage should overlap, the recall between Scopus and WoK varies, as can be seen in Table 6.1. Relative to the Scopus search, WoK has an additional six hits from articles in these journals: Acta Paleontologia Polonica, Apidologie, Coleopterists Bulletin, Geodiversitas, Journal of Biogeography, and Journal of Vertebrate Paleontology (full article bibliographic data for these Scopus and WoK 'winclada' searches are provided online (Mounce 2013c,d) and on the CD provided with the hard copy of this thesis. These six missing articles do contain the word 'winclada' in the abstract and all six journals are indexed in Scopus according to their list of indexed titles as of April 2013 (Sciverse, 2013). It is unclear why Scopus cannot find the word winclada in these six article abstracts and thus does not return them in its results, which it should be able to find. Thus for the winclada search Scopus has a recall of at best $66 \%$ (12/18) for abstract search, assuming perhaps unsafely that WoK finds all the relevant article abstracts that it indexes (?unknown). Scirus finds $16 \%$ of what GS finds for the winclada search and this perhaps reflects the narrowness of the biological journal sources that Scirus restricts itself to - just ten relevant to biology: Elsevier, Wiley, Springer, BMC, the PMC OA full-text subset (which includes all PLOS journals), OUP, CUP, NPG, Royal Society \& Hindawi.

\subsubsection{Scirus does not cover many important natural history journals}

This restricted range of sources used by Scirus search makes it unsuitable for phylogenyrelated searches. Relative to the range of sources searched by WoK (albeit abstract-only in this database), I find that at the article level - the unit that counts in these matters - the ten full-text sources searched by Scirus, listed above account for only $\sim 61 \%$ of nearly 10,000 phylogeny-related articles published in the year 2010 , found and classified by publisher from a search of WoK that I performed as part of an international collaboration (Stoltzfus et al. 2012, detailed source data for this estimate in Mounce 2013e ].

Non-biomedical phylogenetic content included in WoK but likely to be outside of the Scirus full-text search capability includes: AAAS (Science), Academica Sinica (Botanical Studies, 
Zoological Studies), Allen Press (Herpetologica, Journal of Mammology, Phycologia), AMNH press (Bulletin of the AMNH, AMNH Novitates), ASIH (Copeia), ASPT (Systematic Botany), CSIRO journals, Magnolia Press (Zootaxa, Phytotaxa), Taylor \& Francis (Journal of Vertebrate Palaeontology, Journal of Systematic Palaeontology, Journal of Natural History). Thus Scirus lacks coverage of many key journals for morphology-based phylogenetic analyses.

\subsubsection{The long-tail of phylogenetic content}

The distribution of $21^{\text {st }}$ century phylogeny-related articles across publishers and journals is remarkably long-tailed. Surprisingly, using the methods of Stoltzfus et al. (2012) one can infer that Zootaxa (a primarily taxonomic journal not especially known for phylogenetics), might now be the $6^{\text {th }}$ largest container of 21 st century phylogeny, just behind PLoS ONE (as shown in figure 6.1).

Distribution of phylogen* articles 2000-2011
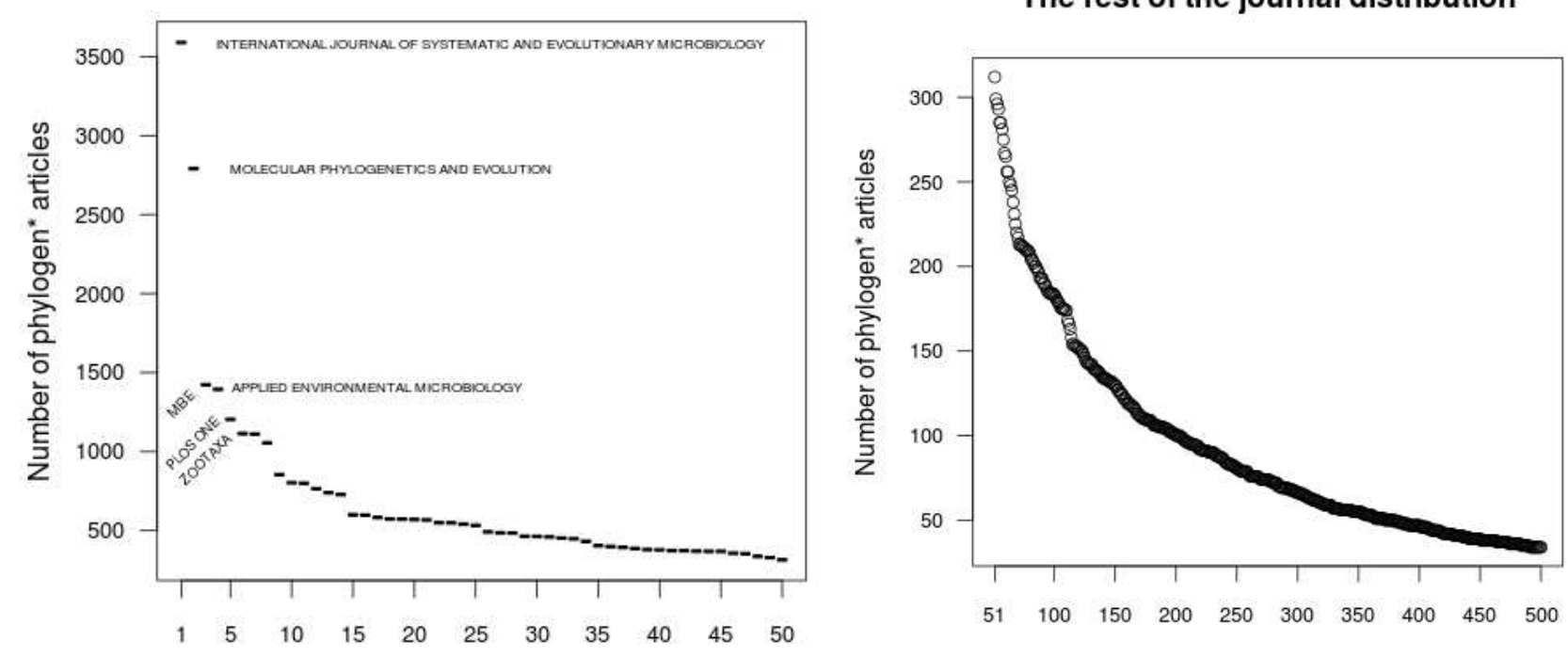

Top 50 journals containing phylogen*

Journals 51 to 500

Figure 6.1 Illustrating the long-tail distribution of phylogeny-related articles across hundreds of different journal titles indexed in WoK, from 2000-2011. Full corresponding data is supplied in the supplementary materials and online (Mounce 2013f). 
There are two important things that figure 6.1 highlights:

- Publishing trends have changed. Now a significant proportion of academics are choosing to publish in megajournals like PLOS ONE and Zootaxa instead of lowvolume traditional journals (this is no bad thing in my opinion).

- Aside from those 6 or 7 journals estimated to be publishing a lot of phylogenetic content - the distribution of the thousands of other phylogenetic articles published each year is remarkably long-tailed. Phylogenetic articles are scattered across at least 1000 journals at a minimum estimate (Stoltzfus et al. 2012 data)

\subsection{Methods}

In order to rigorously examine the capabilities of traditional title-abstract-keyword databases WoK \& Scopus for finding morphology-based phylogenetic analyses published this century, I compared their precision and recall to three different defined corpora of journal articles to which I have full text local desktop access:

A) 'Zootaxa'. The entire set of articles published in the journal Zootaxa from 2001 up to Issue 3690 (1) [11 ${ }^{\text {th }}$ June 2013] inclusive, consisting of 12490 PDF files downloaded direct from the publisher website: http://mapress.com/zootaxal . This set notably includes both large monographs and small erratum notices (see Figure 6.2 overleaf).

B) 'PLoS'. All articles published across seven PLoS journals: One, Biology, Computational Biology, Genetics, Medicine, Neglected Tropical Diseases, and Pathogens from 2003 to 2010-06-04, consisting of 20694 articles obtained via BioTorrents (Langille \& Eisen, 2010).

C) 'BMC'. A complete set of 7948 open access articles containing the stemword 'phylogen*' from 166 journals that BioMed Central publish (2000-2011, full details of each and every article are provided [Mounce, 2013a])

The corresponding PDF's of each corpus were placed in separate self-contained folders. Within these folders I used basic unix command-line tools e.g. pdftotext \& grep searches to identify articles that are very likely to contain relevant content, and then manually scanread the articles myself to classify each search hit. Thus I can be very confident of the actual word content of all the articles in these corpora, relative to the search results provided by the various academic search providers. 


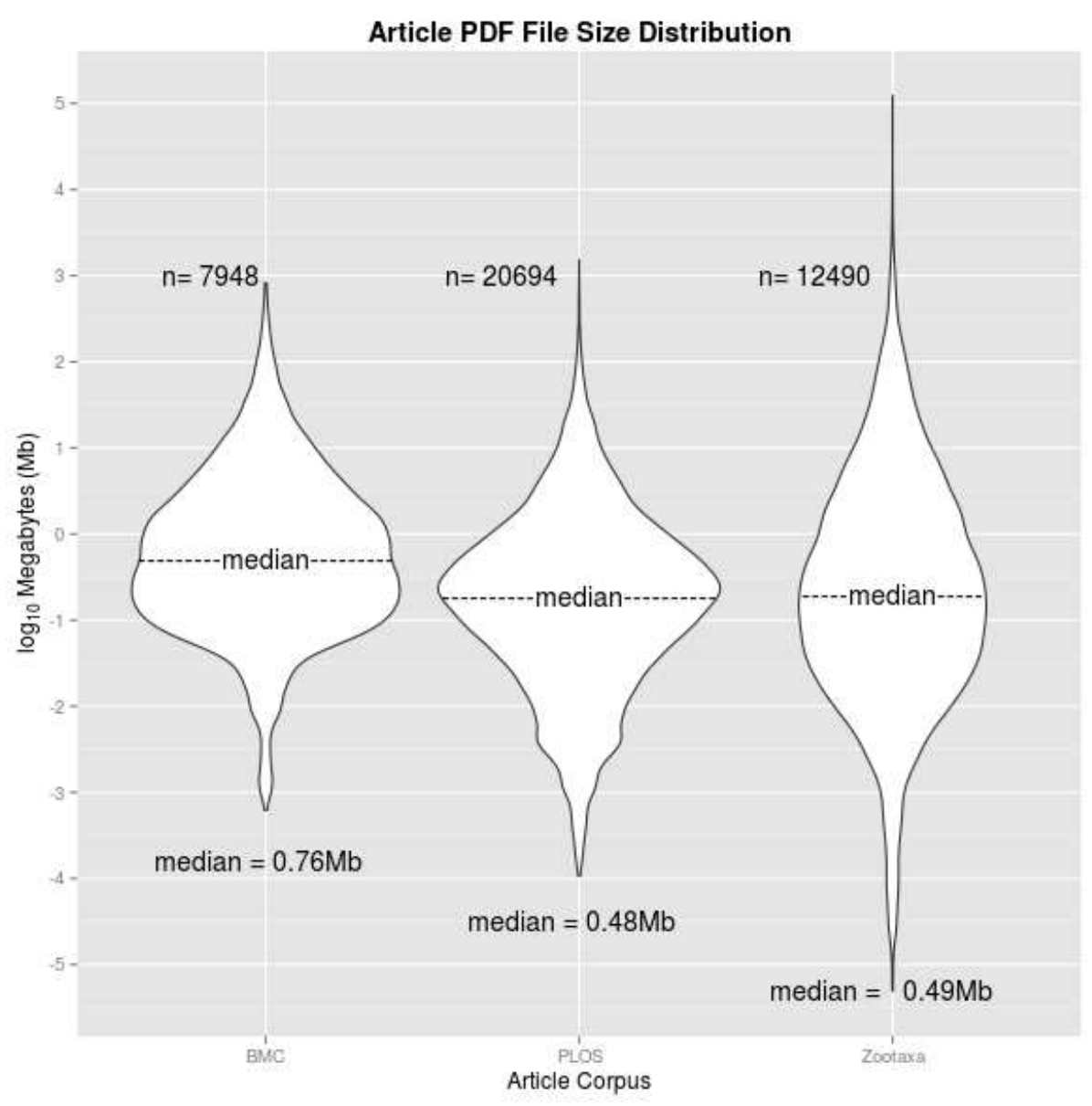

Figure 6.2 Article PDF file size distribution of all articles in each of the corpora. Mean file size across all three corpora is almost exactly 1MB (Mounce 2013b).

\subsubsection{Testing the computational efficiency of local full-text search options}

I speed-tested three different command-line methods to determine which search methods were the quickest in terms of real time. Table 6.2 (overleaf) shows that of the methods that work from 'cold' - spawning embarrassingly parallel grep processes on each of the three cores available returned results in the quickest overall real time. GNU parallel [21] did not speed-up the process because the I/O rate of hard drive access was the limiting factor and this is limited to single-thread access [22]. If one scaled-up this experiment and searched across multiple different disk drives, or used solid state drives (SSD's) GNU parallel may well scale better. Using Recoll [23] returns search results across the entire corpus near instantaneously. But it requires many hours to index all the article content beforehand before searches are performed, so is not truly comparable. Recoll indexing only needs to be done once however to gain this speed benefit for all searches and comes with the 
advantage of a simple GUI interface to perform searches and explore the results.

Table 6.2: A demonstration of different local desktop full text search methods, as performed on the Zootaxa corpus, searching for the term 'winclada' on a simple Phenom X3 2.3Ghz desktop computer, with all files placed on a single HDD.

\begin{tabular}{|l|l|c|c|c|}
\hline \multicolumn{1}{|c|}{$\begin{array}{c}\text { Method } \\
\text { name }\end{array}$} & Real time (s) & $\begin{array}{c}\text { User time } \\
\text { (s) }\end{array}$ & $\begin{array}{c}\text { Sys time } \\
\text { (s) }\end{array}$ \\
\hline 'Parallel grep' & $\begin{array}{l}\text { time find . -name "*.txt" -print0 | xargs -0 -n 1000 } \\
\text {-P 3 grep -iRI "winclada" > out.tzt }\end{array}$ & 15.989 & 44.163 & 0.384 \\
\hline $\begin{array}{l}\text { 'Single } \\
\text { process grep' }\end{array}$ & time grep -iRI 'winclada' . > out.tzt & 42.993 & 42.803 & 0.180 \\
\hline $\begin{array}{l}\text { using GNU } \\
\text { parallel }\end{array}$ & $\begin{array}{l}\text { find . -type f | time parallel -j+0 'grep -il winclada } \\
\{\} \text { ' }>\text { out.tzt }\end{array}$ & 61.11 & 34.47 & 0.481 \\
\hline using Recoll & \multicolumn{1}{|c|}{ (after indexing all the PDFs overnight) } & $<1$ & n/a & n/a \\
\hline
\end{tabular}

\subsubsection{Justification of search terms used, and the use-case}

Phylogenetic analyses are computationally complex and thus almost always involve the use of a specialised computer program, of which there are few in existence: e.g. PAUP* (Swofford 2002), Winclada (Nixon 2002), NONA (Goloboff, 1999b), TNT (Goloboff et al 2008), Hennig86, MEGA, POY, PHYLIP, Phyml, Garli, RAxML, MrBayes, PSODA. A wellreported paper thus must make reference to one or more of these programs in the full text of the work - this offers a nicely constrained starting point for searches. For this chapter I ignored POY, Garli, MEGA, RAxML, Phyml, and MrBayes as they are never or rarely used in morphology-based analyses. PSODA (Carroll et al. 2007; Carroll et al. 2009) seems to have only been cited 3 times according to GS and thus can also be safely discarded. That leaves just six terms and their variants to search for in the quest for morphologyphylogenies: PAUP*, Winclada, NONA, TNT, Hennig86, and PHYLIP.

I proceeded to use those same search terms (and more) to try and re-find the identified content in the three publisher-delimited corpora using many different academic search providers, benchmarking for recall against the known location of the searched-for terms, in articles, in the corpora. 


\subsubsection{Distinguishing between morphology-based \& molecular-only analyses}

Some of the literature search complexity for morphology-based phylogenetic articles is caused by linguistic complications. Occasionally papers exclusively refer to such morphology-based analyses as cladistic analyses (this terminology is entirely correct, but it makes it difficult to find the paper if one is only searching for the word 'phylogeny' and its variants). Far from being a theoretical occurrence there are many easily documented occurrences of this in the Zootaxa corpus. One can automatically identify papers which mention 'cladist' and NOT 'phylog*' with this simple grep: find . -name "*.txt" -print0 | grep -iR -I 'cladist' | xargs -n 1 grep -iL 'phylog' . There are many papers which merely contain one but not the other stem-word (and no cladistic/phylogenetic analysis), but also more seriously there are papers such as Dimitrov \& Ribera (2005) in which a morphology-based cladistic analysis is reported and nowhere in the paper; not in the title, keywords, abstract, main text, figure captions or reference list does any variant of the stem-word phylog* occur - quite an achievement really. The inverse condition is also true, there are many more papers in which morphology-based phylogenetic analyses are performed with no mention anywhere in the paper of either cladi $^{*}$ or cladogra* (at least 47 different papers in the Zootaxa corpus, in which morphology-based phylogenetic analyses are performed). This further justifies the approach of trying to find analyses by other means (e.g. the name of the software used).

For the purpose of this chapter "relevant content" and "morphology-based phylogenetic analyses" are defined as papers in which new (biological) morphological-character-based phylogenetic analyses are both calculated and reported in the paper - hypothesised figures (only), or reproduction of a phylogenetic tree(s) from previous studies, or 'supertree' analyses, or phenetic analyses (e.g. UPGMA) of continuous measurements do not count. Analyses that combine morphological and molecular data to build the phylogeny are included in the count. Molecular (only) phylogenetic analyses are not defined as relevant content for the purpose of this exercise, which makes the task more difficult since molecular and morphology-based analyses often use the same methods, software and linguistic reporting style. Moreover there are far more published molecular-only papers which hampers the precision of any literature search for morphology-only phylogenetic papers. 


\subsection{Results}

Based on the initial grep for just 'paup' it was determined one could safely exclude some false positives that kept appearing (i.e., the author name 'Paupy' (exclude -y) , the word depauperate (exclude -e), 'paupar-rash', 'paupal wing', 'Paupard' and 'Pauparding-Tritsch' (exclude -a) 'pAUPR' (exclude -r) 'Pauphalictus' (exclude -h)). Searches for 'Winclada' varied in their capitalization of the word but otherwise it was easy to find with $100 \%$ precision. 'Hennig86' was also referred to as 'Hennig 86' and that was easy to deal with. Some authors called TNT: 'TnT' or 'T.N.T.' thus these variations were incorporated into the search. Lots of other non-related concepts were unsurprisingly detected for 'TNT' so that search had low precision in the PLoS corpus. Finally NONA was also problematic for precision; some authors called it 'Nona' and this is harder to distinguish from the rice cultivar 'Nona Bokra' without special rules. Likewise, 'Nona' appeared as an author first name several times in PLoS papers, but I could not easily devise a safe general rule to exclude these false positives (Table 6.3).

Table 6.3: Summary of local desktop full-text searches. Most searches find references to intended software with $100 \%$ precision (no false positives).

\begin{tabular}{|c|c|c|c|c|c|}
\hline Base Term & Corpus & Essence of Method & $\begin{array}{l}\text { Total } \\
\text { Hits } \\
\text { (article) }\end{array}$ & $\begin{array}{l}\text { Hits are } \\
\text { intended } \\
\text { concept } \\
(\%)\end{array}$ & $\begin{array}{l}\text { Morph- } \\
\text { based } \\
\text { analyses }\end{array}$ \\
\hline \multirow[t]{3}{*}{ paup } & BMC & grep -iR -I "paup[^ehyar]" & 896 & 100 & 10 \\
\hline & PLOS & & 293 & 100 & 8 \\
\hline & Zootaxa & & 689 & 99.9 & 286 \\
\hline \multirow[t]{3}{*}{ winclada } & BMC & grep -iR -I "winclada" & 7 & 100 & 1 \\
\hline & PLOS & & 3 & 100 & 0 \\
\hline & Zootaxa & & 151 & 100 & 96 \\
\hline \multirow[t]{3}{*}{ hennig86 } & BMC & grep -iR -I "hennig86||hennig 86" & 1 & 100 & 0 \\
\hline & PLOS & & 0 & $\mathrm{n} / \mathrm{a}$ & 0 \\
\hline & Zootaxa & & 27 & 100 & 26 \\
\hline \multirow[t]{3}{*}{ nona } & BMC & $\begin{array}{l}\text { grep -R -I "Nona[^A-Za-Z]||NONA[^A-Za- } \\
\text { z]" }\end{array}$ & 10 & 90 & 3 \\
\hline & PLOS & & 12 & 16.6 & 0 \\
\hline & Zootaxa & & 103 & 96.1 & 88 \\
\hline \multirow[t]{3}{*}{ tnt } & BMC & grep -iR -I "tnt[^A-Za-z]||tl.nl.t" & 7 & 57.1 & 2 \\
\hline & PLOS & & 190 & 7.9 & 5 \\
\hline & Zootaxa & & 151 & 100 & 105 \\
\hline
\end{tabular}




\begin{tabular}{|l|l|l|c|c|c|}
\hline phylip & BMC & grep -iR -I "phylip[^A-Za-z]" & 912 & 100 & 1 \\
\hline & PLOS & & 241 & 100 & 0 \\
\hline Zootaxa & & 22 & 100 & 6 \\
\hline
\end{tabular}

If desired one can combine all the search terms with the boolean operators e.g. OR which in grep syntax is V e.g. hennig86/|winclada. One can also print lines of context around the hit line to aid quick classification of the paper without having to open/read the original PDF. For example, with -A and -B in grep one can specify the number of lines of context to show above and below the line of interest. Samples of this output are given in Appendix 6.1.

Additional searches revealed that just looking in the reference list for references to the given software is not a safe, conservative strategy. For example:

find . -name "*.txt" -print0 | xargs -0 -n 1000 -P 3 grep -iR "goloboff" | grep -i "nixon" as applied to the PLoS corpus found only 11 reference variants to "Goloboff PA, Farris JS, Nixon K (2003) TNT...", and the year given varied from 2000-2008. The more thorough fulltext search strategy for TNT given in Table6.3 gave many more false positives, but importantly it identified 15 instances in the PLoS corpus where TNT was used. Why the discrepancy? Manual examination of the four papers in which TNT was found but a citation including 'Goloboff' \& 'Nixon' could not, demonstrated that:

- In Deo et al (2010) TNT was mis-cited as just "Goloboff PA (2000) TNT (Tree analysis using New Technology). 1.2 BETA ed. Tucuman, Argentina: By Authors." Although primarily Goloboff's work, the official history of the program shows it has always been a collaborative work between Goloboff, Nixon \& Farris. Also given the submission date of this paper (2009-11-24) I see no reason why (Goloboff et al. 2008) describing TNT was not cited.

- In Wilson (2010) TNT was misattributed to another paper by Goloboff: "Goloboff PA, Catalano SA, Mirande JM, Szumik CA, Arias JS, et al. (2009) Phylogenetic analysis of 73060 taxa corroborates major eukaryotic groups. Cladistics 25: 211230"

- In Dilernia et al 2008 TNT was just referred to in the main text as "available at http://www.zmuc.dk/public/phylogeny/TNT)" which does not give proper attribution (WoK only counts citations given in the reference list).

- In Phillips et al (2010), TNT was misattributed to another paper by Goloboff: “Goloboff PA, Farris JS, Källersjö M, Oxelman B, Ramírez MJ, et al.. (2003) 
Improvements to resampling measures of group support. Cladistics 19: 324-332".

Table 6.4 Comparison of recall between popular academic web literature search portals, to the known number of articles that contain the search term in the full text of the article.

\begin{tabular}{|c|c|c|c|c|c|c|c|c|}
\hline Journal & Period (inclusive) & Searched terms & $\begin{array}{l}\text { MAS } \\
\text { hits }\end{array}$ & $\begin{array}{l}\text { MS } \\
\text { hits }\end{array}$ & $\begin{array}{l}\text { WoK } \\
\text { hits }\end{array}$ & $\begin{array}{l}\text { Scopus } \\
\text { hits }\end{array}$ & $\begin{array}{l}\text { GS } \\
\text { hits }\end{array}$ & $\begin{array}{l}\text { Local } \\
\text { grep hits }\end{array}$ \\
\hline Zootaxa & 2001 to $2013-06-11$ & hennig86 OR hennig 86 & 0 & 0 & 0 & 13 & 10 & 25 \\
\hline Zootaxa & 2001 to $2013-06-11$ & paup & 0 & 2 & 6 & 444 & 332 & 705 \\
\hline Zootaxa & 2001 to $2013-06-11$ & Nona OR NoName & 0 & 2 & 5 & 75 & 49 & 90 \\
\hline Zootaxa & 2001 to $2013-06-11$ & TNT OR T.N.T. & 0 & 3 & 8 & 108 & 61 & 148 \\
\hline Zootaxa & 2001 to $2013-06-11$ & phylip & 0 & 0 & 0 & 14 & 8 & 21 \\
\hline Zootaxa & 2001 to $2013-06-11$ & winclada & 0 & 0 & 0 & 105 & 51 & 140 \\
\hline Zootaxa & 2001 to $2013-06-11$ & phylogeny & 0 & 292 & 1650 & 3903 & 2420 & 4596 \\
\hline Zootaxa & 2001 to $2013-06-11$ & phylogen* & 0 & $\mathrm{n} / \mathrm{a}$ & 2104 & 5561 & $\mathrm{n} / \mathrm{a}$ & 6804 \\
\hline Zootaxa & 2001 to $2013-06-11$ & phylog* & 0 & $\mathrm{n} / \mathrm{a}$ & 2136 & 5618 & $\mathrm{n} / \mathrm{a}$ & 6884 \\
\hline $\begin{array}{l}\text { PLOS } \\
\text { ONE }\end{array}$ & 2006 to 2009 & hennig86 OR hennig 86 & 0 & 0 & 0 & 0 & 0 & 0 \\
\hline $\begin{array}{l}\text { PLOS } \\
\text { ONE }\end{array}$ & 2006 to 2009 & paup & 50 & 0 & 0 & 33 & 130 & 131 \\
\hline $\begin{array}{l}\text { PLOS } \\
\text { ONE }\end{array}$ & 2006 to 2009 & Nona OR NoName & 1 & 0 & 0 & 1 & 8 & 6 \\
\hline $\begin{array}{l}\text { PLOS } \\
\text { ONE }\end{array}$ & 2006 to 2009 & TNT OR T.N.T. & 10 & 2 & 2 & 7 & 81 & 84 \\
\hline $\begin{array}{l}\text { PLOS } \\
\text { ONE }\end{array}$ & 2006 to 2009 & phylip & 58 & 1 & 1 & 20 & 99 & 100 \\
\hline $\begin{array}{l}\text { PLOS } \\
\text { ONE }\end{array}$ & 2006 to 2009 & winclada & 0 & 0 & 0 & 0 & 2 & 2 \\
\hline $\begin{array}{l}\text { PLOS } \\
\text { ONE }\end{array}$ & 2006 to 2009 & phylogeny & 257 & 507 & 521 & 774 & 680 & 678 \\
\hline $\begin{array}{l}\text { PLOS } \\
\text { ONE }\end{array}$ & 2006 to 2009 & phylogen* & 9 & 2647 & 618 & 1087 & $\mathrm{n} / \mathrm{a}$ & 1385 \\
\hline $\begin{array}{l}\text { PLOS } \\
\text { ONE }\end{array}$ & 2006 to 2009 & phylog* & 0 & 2768 & 624 & 1105 & $\mathrm{n} / \mathrm{a}$ & 1399 \\
\hline
\end{tabular}

Table 6.4 gives the results of the recall tests for term searches between various academic search providers, compared against the known number of relevant article hits for that term in each journal (known from the local full-text searches, manually evaluated as part of work shown in Table 6.3). Example queries for each academic search provider are given in Appendix 6.2 for the purpose of transparency and reproducibility. 
GS found most of the articles containing the searched-for terms in the open access journal PLoS One. In the case of the search for 'Nona' the discrepancy was caused by some false-positives returned: it finds three false-positive articles which have these words in them: "nona-L-arginine", "nona-arginine" and "nonA". My local full-text search for 'Nona/NONA/NoName' (Table 6.3) used a more sophisticated case-sensitive search to provide more precise results. This level of sophistry of search is not provided by any of the web-based academic search providers. The GS search results for Zootaxa, on average less than $44 \%$ recall, are significantly different in recall relevant to its performance against PLoS One, which had near $100 \%$ recall. Closer examination of the returned results for the GS Zootaxa searches show that the results returned often correlated very significantly with if the full-text of the paper had been deposited freely online at an institutional web address (see Appendix 6.3 for an example). MAS also showed a similar pattern of recall against PLoS One articles, presumably because MAS operates similarly to GS by crawling the web. By raw volume of returned results Scopus appears to be the best search provider for searching Zootaxa, but I would urge caution in this assessment because I did not check the precision of returned results in most cases, so these numbers of returned results could represent many false positives, the extent to which I do not know.

WoK unsurprisingly given it searches just titles-abstracts-keywords, for the specific software/methodological terms, retrieved hardly any articles but fared a little better for more general terms such as 'phylogeny', averaging just above 30\% 'recall' if one generously assumes that none of the returned results were false positives. For the same general 'phylogeny' search terms Scopus had a much higher 'recall' averaging above $80 \%$, again assuming no false positives.

\subsection{Discussion}

\subsubsection{What exactly is WoK missing? Does it matter?}

To determine the implications of WoK's apparent low recall against Zootaxa for general terms like phylogeny I sought to find and attempt to explain why it misses so many articles in which the word 'phylogeny' occured. To do this I manually examined the search results returned for the maximally-conservative search for 'phylog*' in Zootaxa by WoK for the 
years 2005-2006 inclusive, which returned 172 articles (data in supplementary materials). I then scored if the term occurred in each of the articles in the abstract+title+keywords, main full text (excluding abs-title-key), and/or references.

Curiously, 45 of the WoK-found articles did not contain the search term phylog* in the abstract, title, or keywords (rather it was in the main text or references). Stranger still, of these 45, nine only had the searched-for term in the reference list at the end of the paper (Bray \& Cribb 2005; Martin 2006; Samyn et al. 2006; Kajihara 2006; Sterrer 2006; Velez et al 2006; Dozsa-Farkas \& Cech 2006; Winterton 2006; Craig et al 2006), which I would contend hardly makes the article of likely relevance to the initial search term.

I also checked the complement of articles that can be found to match 'phylog*' from a local desktop grep search, which returned 291 articles published in 2005, and 542 articles published in 2006. Of the 291 articles from 2005, 98 represented matches to a word or words in the reference-list only. This careful search of 291 articles published in 2005 revealed six articles (Li et al. 2005; Betancur-R \& Acero 2005 ;Edgecombe \& Hollington 2005; Sharkey 2005; Webb 2005; Ponder et al 2005) which contained new phylogenetic analyses reported in the paper that were not found by WoK searches for phylog*, phylogen* or phylogeny. I did not have time to manually search all 542 of the articles from 2006 that grep finds, but a cursory search also finds that WoK does not find Bravo (2006) because the language used in the abstract refers consistently to 'cladistics' rather than phylogenetics. An additional search for 'cladis*' in WoK only additionally finds Ponder et al (2005) and Bravo (2006), not the others.

This is highly significant as it demonstrates that a search strategy of relying on 'phylog*' OR 'cladis*' searches in WoK will not find at least 5 of the phylogenetic analyses $(>10 \%$ of those published that year) that should be in the journal scope of WoK, let alone all the phylogenies ever published in all peer-reviewed journals.

\subsubsection{What about the non-journal data archives? Do they have the data?}

What of the phylogenetic data archives Treebase, Morphobank, and the more general data archive provided by Dryad? Could they be of help in finding phylogenetic data? Treebase 
has data for 27 phylogenetic studies published in Zootaxa (http://treebase.org/treebaseweb/search/studySearch.html?query=prism.publicationName $==\% 22 Z$ Zootaxa $\% 22$ data as accessed 2013-07-24 ). A simple grep of the Zootaxa corpus reveals 35 papers containing the string 'treebase' but interestingly these do not all match-up to the 27 in TreeBASE (data supplied in electronic supplementary materials:

https://github.com/rossmounce/thesis_ESM/blob/master/lit_search_chapter/treebase_ther e_and_not.csv ). One hit comes from the phrase 'treebased assessment'; nothing to do with TreeBASE. Another describes the TreeBASE project (Maddison et al. 2007). Another hit comes from Guayasamin et al. (2009) re-using published data in TreeBASE. In fact only 17 of those 35 'treebase' mentions in Zootaxa actually deposited their primary data in TreeBASE. There are seven studies (Triapitsyn et al. 2006; Harmer \& Framenau, 2008; Kerr, 2010; Nygren et al. 2010; Brix et al. 2011; Prentice \& Redak, 2012; Ballantyne \& Lambkin, 2013;) which claim in the paper to have deposited data in TreeBASE, for which no corresponding public data can be found actually in TreeBASE. I do not know the reason behind this discrepancy but I have sometimes stumbled upon examples of this before with other papers in other journals during my PhD (e.g. Peach \& Rouse (2004) which also claims to have associated deposited data in TreeBASE).

\subsubsection{Few morphology-using phylogenetic analyses in BMC journals}

The entire output of BMC published/owned journals from 2000-2011 contain just thirteen phylogenetic analyses derived (entirely or in part) from morphological data (Wagele \& Kolb 2005; Asher 2007; Seiffert 2007; Haug et al. 2010; Zhao et al 2010; Struck 2007; Ahrens \& Ribera 2009; Reidenbach et al 2009; Zrzavy et al 2009; Jenner et al 2009; Asher et al 2010; Pepato et al 2010; Geisler et al 2011) (the last eight of these are combined morphology and molecular analyses). The articles in BMC Evolutionary Biology \& Frontiers in Zoology often required manual examination to determine. There are other studies that are closely related but not quite what I was seeking (e.g. numerous supertree studies, the phenetic UPGMA analysis of morphological measurements in Froufe et al. (2008), and Puniamoorthy et al. (2010) who generated a discrete character matrix but choose to map it onto pre-existing phylogenetic hypotheses). These studies all warranted close examination.

Applying the same approach on the BMC corpus did not find much, but was certainly worth doing: BMC publishes several general biology journals (e.g. Biology Direct, Journal of 
Biology, BMC Research Notes, BMC Biology). It is conceivable that a relevant study could have been published in these journals, even if I subsequently found that not to be the case

- the negative is worth establishing as much as the positive. The exercise also gave me a chance to discover interesting uses of cladistic methods in other disciplines e.g. Anthropology (Lycett, 2009).

Having done this analysis it would be remiss of me to ignore the bigger picture. Even with these full text methods, searching method sections written in natural language is harder than it needs to be. I would suggest that these phylogenetic method sections could easily be supplied as machine-readable statements using a controlled vocabulary of terms according to a reference standard (e.g. MIAPA [Leebens-Mack 2006]). The phylogenetic programs themselves could export the method used in an unambiguous, fully-explicit (no unmentioned hidden parameters) machine-readable format along with (or separate from), the data analysed with that method. In fact many phylogenetic programs already can export the data together with the results and the method, but for various reasons journals/authors/editors choose not to publish these in a re-usable, machine-readable form most of the time (Stoltzfus et al. 2012). Workflow tools like Armadillo (Lord et al. 2012) have also tried to enable reproducibility in phylogenetics by allowing researchers to save \& record their entire workflow for prosperity and transparency but it remains seldom used so far (GS: 1 citation). The printed page should not and need not restrict what we do with scientific data. A multitude of high-quality options are available to authors who wish to make their data more easily re-usable including Dryad, Figshare, MorphoBank, Morphbank, and TreeBASE.

\subsection{Conclusions}

Whilst in other academic domains, rigorous full-text searches are the norm (e.g. medical sciences, c.f. The Cochrane Reviews), in ecology, systematics and evolution, rigorous approaches to literature search are seldom applied. This is partly because there is not adequate infrastructure (e.g., PubMed) to facilitate full-text full-corpus searching for the entire literature of this domain. Also it is prevented by legal restrictions and download rate limits that certain subscription access publishers place on 'their' material, making it very 
hard to download all of it (Mounce, 2013). However, there is huge potential for use of fulltext analysis in this area.

There are no longer technical barriers to large-scale data synthesis/mining in this area. I estimate, using Thomson Reuters JCR data, that the average journal in the areas of systematics, ecology and evolution publishes approximately 60 papers each year (median), and that a representative random sample of 200 journals from this subject area published cumulatively 17,000 articles last year [see Appendix 6.4]. If one scales this up this estimate to a 1,000 journals, from 2000-2013, using the mean PDF size given in 6.2 then the entire 'born-digital' literature for this area is likely to be well-less than 1.2 Terabytes (for perspective: commercially-available 3TB hard drives cost less than $£ 80$ as of 2013, with prices almost certain to decrease over time). This can of course be minimized further by just utilizing a HTML, XML or plain-text version rather than the PDF, but it shows one could easily archive all of the data on a standard desktop computer. 


\section{Chapter 7: Lost Branches in the Fossil Tree of Life}

\subsection{Introduction}

In this concluding chapter I reflect-on and evaluate my experience of trying to find, reextract, validate, and re-use published palaeomorphological data from the published literature. As I quickly found out during my research this is not an easy task and as such I feel a duty to report these findings in my thesis because I think my conclusions here are of real importance to future research efforts. To reconstruct some data sets from the published literature, the effort required is akin to that of fossil preparator delicately removing matrix from fossil bone - data is often difficultly 'embedded' in old scanned-in PDFs - one cannot just lift data out from these at the click of a button. Likewise, as fossil specimens are commonly found in a disarticulated and fragmentary state; the same is also true of palaeomorphological data in the literature. To 'save space' in the printed version of research publications much data is still commonly omitted, with only the newly added data being shown (and this is often replicated in the electronic version of the article). Yet to reanalyze or build on this data set one needs the complete data set. As I show in this chapter, this fragmented data availability is both unnecessary (the online version of the paper has no 'space' constraints other than file size, which the extra kilobytes of phylogenetic data should not trouble) and a significant hindrance. Emailing authors to ask them for a usable copy of their published data does not often result in a successful outcome. Drew et al. (2013) report that only $16 \%$ of 375 authors contacted, actually replied with the desired phylogenetic data. My own attempts at asking authors for their published data have had a similarly low success rate, although I did not care record the successes and failures in my inbox. However, I remain immensely grateful to those authors who do supply they published data upon request, or those who deposit their data online in an easily discoverable data repository like Dryad, TreeBASE or MorphoBank. 


\subsection{How many morphology-based phylogenetic studies are there?}

The question I ask in this sub-heading is extremely important to establish as a baseline. Given that fossils and morphology are important to include in phylogenetic research (Chapter 1, Chapter 3) - how much of that type of research is there? Work that I contributed to; specifically the literature search and analysis of Stoltzfus et al. (2012) crudely demonstrates that around two thirds of papers that have the stemword "phylogen*" in them, as found by Web of Knowledge, report a phylogenetic analysis. Extrapolating this two thirds proportion to other publication years demonstrates that there are as a conservative estimate, over 100,000 published phylogenetic analyses in the modern 21st century peer-reviewed literature (2000-2012). Whilst the proportion of these that are morphology-based, or morphology-using (in conjunction with molecular data) is likely to be low, even then it should be well into the thousands. As I show in chapter 6, reliably discriminating between molecular and morphology-based phylogenetic analyses using currently available literature search techniques is extremely difficult. However, more sophisticated approaches using text and data mining techniques on full-text literature corpora may prove useful in this regard and it is an avenue of research I am actively pursuing thanks to the Panton Fellowship awarded to me by the Open Knowledge Foundation (Newman, 2012).

Throughout the last 4 years, I have been tagging wholly morphology-based vertebrate and invertebrate phylogenetic studies online, so that I have openly available bibliographic records of when and where they occur (Mounce 2013g,h). Reconciliation between the bibliographic data I hold, and that of Graeme Lloyd's bibliographic data on phylogenetic studies (Lloyd, 2009) shows that at a bare minimum, for the period 2000-2012 inclusive there are more than 3,800 peer-reviewed, published, morphology-based phylogenetic studies (at a bare minimum, I expect there are perhaps more than 5,000 if one also includes botanical studies which neither I nor Graeme Lloyd cover in much depth).

\subsection{How much data from morphology-based phylogenetic studies is publicly available?}

Given my estimate of 3,800 to $>5,000$ studies out there somewhere in the literature, 
scattered across many hundreds of different journals (figure 6.1), a question that is easier to answer more definitively is: how many morphology-based phylogenetic studies are there in publicly available data repositories for immediate re-use? Thanks to William Piel (pers. comm.) there is a TreeBASE (Piel et al. 2002) query one can run that shows all morphology-using studies in TreeBASE:

http://treebase.org/treebase-web/search/matrixSearch.html? query=tb.type. matrix=Morphological\%20or\%20tb.type. matrix=Combination\%20or \%20tb.type.matrix=Behavior

Currently (2013-10-01) there are 784 matrices in TreeBASE found by this query, from 646 individual studies. 433 of the studies were published between 2000-2012. 635 of these matrices are solely morphological, 147 are combined data sets of morphology and molecules, and 2 are matrices of behavioural data. Note that for some, there's more than one matrix in TreeBASE corresponding to a single study.

Dryad (http://datadryad.org/) also archives phylogenetic data in a publicly available, reusable format. It can pass-on data initially submitted to Dryad, to TreeBASE, so there is some duplication of content between the two. It is harder to search for purely morphological phylogenetic data sets in Dryad as it is a generic datastore for all sorts of biological data, but nevertheless I identify just 12 data sets in Dryad that are not yet in TreeBASE.

Morphobank (http://www.morphobank.org/ ; O'Leary and Kaufman, 2011) has 249 publicly available projects available as of 2013-10-01 but not all of these projects have an associated morphology-based phylogenetic data matrix. 218 of these represent studies from 2000-2012.

Thus between the big three publicly-accessible databases suitable for this type of data have morphological data from approximately 663 individual studies (assuming no duplication between Morphobank \& TreeBASE content which is perhaps an unsafe assumption), for studies from the period 2000-2012. As I estimate there are comfortably 5000 such morphological studies in the literature from that time period - I estimate thus that we have programmatic access to data from just $13 \%$ of the studies from this recent period. 
But what of data provided in the article or the supplementary materials? This data is often error-prone (e.g. typesetting errors), ill-formatted, inextractable and difficult to re-use. Nor is it very discoverable (Chapter 6). It is difficult to explain on paper quite how much of a dumping ground supplementary materials files are, so I instead point to my Young Systematists' Forum talk on this very matter for further evidence (Mounce, 2010).

\subsection{Replicating published cladistic analyses}

Interestingly, I have observed many times during my work that the published dataset, and the published methodology of analysis e.g. parameters, character orderings and weightings, do NOT match the published results. Most of the time the differences are small, but for more than just a few papers the difference really is striking and significantly contradicts at least some of the conclusions of a paper.

One such example that I successfully challenged was that of the phylogeny published in a high-profile, front cover Nature article by Liu et al. (2011). The data simply didn't match the reported result no matter what parameters one applies, this was further confirmed by another research group who also independently noticed the analysis was not reproducible (Legg et al. 2011). I include my published article (Mounce \& Wills, 2011) in full, in Appendix 7.1 to further evidence this.

\subsection{Comments on the Liu et al. reply}

The authors of the original article wrote a formal reply (Liu et al. 2011b) to both our reanalysis (Mounce \& Wills 2011) and that of Legg et al. (2011). In this interesting reply they accept many of our criticisms but do not appear to readily admit the main point of Mounce \& Wills (2011) and Legg et al. (2011) that the consensus tree supported by their data is significantly different from the one they initially reported. Figure 1 (Liu et al. 2011b) presents what they purport to be a bootstrap analysis of their data to "verify the stability" of their findings. Bootstrap analyses (Felsenstein 1985) resample characters with replacement. Given their data matrix only has 38 characters most of which are binary, for 28 taxa - it's clear a priori that bootstrap support values are extremely unlikely to be 100 for each and every node of the consensus tree. Alas, I report here that much like their 
previous analyses (Liu et al. 2011a) I could not replicate their bootstrap analysis in either relationships depicted or strength of support for the optimal topology. I can only conclude the people who contributed to the data analysis of both Liu et al. $(2011 a, b)$ have a very different interpretation of cladistics and bootstrap analyses to most other people in the research in community. Similarly they called the PTP test they used the 'partitioning tail permutation' test, yet myself and most others know it as the permutation tail probability test (Faith \& Cranston 1991).

\subsection{Suggestions for the future of data publication and review}

Research data is immensely valuable, including re-use value. Yet current publishing practices don't seem to me to be treating it as such - scarcely any data is made available for re-use as this chapter demonstrates. Most of the focus behind the publication and review process appears to go on the paper and the figures, not the code, data and analyses behind them. I have suggested we should change this (e.g. at the Systematics Association Biennial Meeting; Mounce, 2011b). In morphology-based phylogenetics where the analyses are typically computationally very simple - just seconds to find the consensus tree with TNT for most parsimony analyses - that reviewers should routinely re-run the data to independently check and validate the reported results in such papers. This would hopefully go some way to prevent problems of non-reproducibility like that reported in Mounce \& Wills (2011). Furthermore, if the authors hand-over the raw re-usable data at review for the reviewers to access, it would seem relatively simple to me to ensure that once the article is accepted, that this raw data file also gets made publicly available in an appropriate data repository e.g. TreeBASE, Dryad or Morphobank. This would be a huge improvement on the current jumble of supplementary information files that are rarely immediately re-usable - it is a far cry from the simplicity and utility of Genbank for DNA sequence data. Usually supplementary files are PDF's mixing lots of different types of data in a long document, or a Word file, or even a spreadsheet. By putting data in a data repository it ensures scalable programmatic access and the chance to build rich searchable metadata on top of the data deposit (e.g. as TreeBASE does) to make the data more easily discoverable. Data buried on the 90th page of a supplementary file PDF is effectively lost to re-use to all but the most determined of data re-users. 


\subsection{Positive reasons to share published data}

I would like to end on a positive note. The scholarly publishing landscape is rapidly changing at the moment. The US, UK, Europe and countries around the world are now committed to moving towards open access publishing. Many journals have adopted a mandatory data archiving policy for all authors e.g Evolution (Fairbairn 2011). Research funders are explicitly recognising the value of data and code, not just publications (Piwowar, 2013). Research is demonstrating that data archiving is a worthwhile investment (Piwowar et al. 2011) and that sharing detailed research data is associated with an increased citation rate (Piwowar et al., 2007). The latest results show that papers with open data available with no legal restrictions on its usage receive approximately $9 \%$ more citations than similar studies in which data are not made available (Piwowar \& Vision 2013). I don't doubt that this apparent citation benefit is transferable to palaeontology, systematics, macroevolutionary studies and ecology - data re-use is a common facet of science. Instead of being mandated or 'forced' to share data, perhaps one day researchers will be eager to share data, to their own benefit as well as that of others (Poisot et al. 2013). With publications such as White et al. (2013) providing clear advice and help on making data re-usable, I'm confident that in future we'll have less 'lost branches' (sensu Drew et al. 2013) on the Fossil Tree of Life. 


\section{References}

Aberer, A. J., Krompass, D., and Stamatakis, A. 2013. Pruning rogue taxa improves phylogenetic accuracy: an efficient algorithm and webservice. Systematic Biology 62:162-166.

Adnet, S. and Cappetta, H. 2001. A palaeontological and phylogenetical analysis of squaliform sharks (Chondrichthyes: Squaliformes) based on dental characters. Lethaia 34:234-248.

Adrain, J. M. 2001. Systematic Paleontology. Journal of Paleontology 75:1055-1057.

Ahyong, S.T. 2006. Phylogeny of the clawed lobsters (Crustacea: Decapoda: Homarida) Zootaxa 1109:1-14

Allain, R. and Aquesbi, N. 2008. Anatomy and phylogenetic relationships of Tazoudasaurus naimi (Dinosauria, Sauropoda) from the late Early Jurassic of Morocco. Geodiversitas 30:345-424.

Allard, M.W., Farris, J.S. and Carpenter, J.M. 1999a. Congruence among mammalian mitochondrial genes. Cladistics 15:75-84.

Allard, M.W., Kearney, M., Kivimaki, K.L., Meisner, A.D. and Strong, E.E. 1999b. The random cladist: A review of the software package Random Cladistics. Cladistics 15:183-189.

Allentoft, M. E., Collins, M., Harker, D., Haile, J., Oskam, C. L., Hale, M. L., Campos, P. F., Samaniego, J. A., Thomas, Willerslev, E., Zhang, G., Scofield, R. P., Holdaway, R. N., and Bunce, M. 2012. The half-life of DNA in bone: measuring decay kinetics in 158 dated fossils. Proceedings of the Royal Society B: Biological Sciences 279:4724-4733.

Anderson, J.S., Reisz, R.R., Scott, D., Frobisch, N.B. and Sumida, S.S. 2008. A stem batrachian from the Early Permian of Texas and the origin of frogs and salamanders. Nature 453:515-518.

Andres, B., Clark, J. M., and Xing, X. 2010. A new rhamphorhynchid pterosaur from the Upper Jurassic of Xinjiang, China, and the phylogenetic relationships of basal pterosaurs. Journal of Vertebrate Paleontology 30:163-187.

Apesteguia, S. and Zaher, H. 2006. A Cretaceous terrestrial snake with robust hindlimbs and a sacrum. Nature 440:1037-1040.

Arango, C. P. and Wheeler, W. C. 2007. Phylogeny of the sea spiders (Arthropoda, Pycnogonida) based on direct optimization of six loci and morphology. Cladistics 23:255-293

Archie, J. W. 1989. Homoplasy excess ratios: New indices for measuring levels of homoplasy in phylogenetic systematics and a critique of the consistency index. Systematic Biology 38:253-269.

Archie, J.W. 1996. Measures of Homoplasy pp. 153-188. In: M.J. Sanderson, L. Hufford, editors. Homoplasy: The Recurrence of Similarity in Evolution. Academic Press, New York.

Archie, J.W. and Felsenstein J. 1993. The number of evolutionary steps on random and 
minimum length trees for random evolutionary data. Theoretical Population Biology 43:52-79.

Arratia, G. 2009. Identifying patterns of diversity of the actinopterygian fulcra. Acta Zoologica 90:220-235.

Asher, R.J., Meng, J., Wible, J.R., McKenna, M.C., Rougier, G.W., Dashzeveg, D. and Novacek, M.J. 2005. Stem Lagomorpha and the antiquity of Glires. Science 307:10911094.

Asher, R.J. and Hofreiter, M. 2006. Tenrec phylogeny and the noninvasive extraction of nuclear DNA. Systematic Biology 55:181-194.

Asher, R.J. 2007. A web-database of mammalian morphology and a reanalysis of placental phylogeny. BMC Evolutionary Biology 7:108+.

Asher, R.J., Bennett, N. and Lehmann, T. 2009. The new framework for understanding placental mammal evolution. Bioessays 31:853-864.

Ashlock, P. D. 1974. The uses of cladistics. Annual Review of Ecology and Systematics 5: 81-99.

Austin, J. J., Ross, A. J., Smith, A. B., Fortey, R. A., and Thomas, R. H. 1997. Problems of reproducibility - does geologically ancient DNA survive in amber-preserved insects? Proceedings of the Royal Society of London. Series B: Biological Sciences 264:467474.

Avise, J.C. and Robinson, T.J. 2008. Hemiplasy: a new term in the lexicon of phylogenetics. Systematic Biology 57:503-507.

Babot, J., D. A. López, and T. J. Gaudin 2012. The most ancient xenarthran petrosal: morphology and evolutionary significance. Journal of Vertebrate Paleontology 32:11861197.

Ballantyne, L. A. and Lambkin, C. L. 2013. Systematics and Phylogenetics of Indo-Pacific Luciolinae Fireflies (Coleoptera: Lampyridae) and the Description of new Genera. Zootaxa 3653:1-162.

Baker, R., Yu, X. and DeSalle, R. 1998. Assessing the relative contribution of molecular and morphological characters in simultaneous analysis trees. Molecular Phylogenetics and Evolution 9:427-436.

Bakker, M. and Wicherts, J. M. 2011. The (mis)reporting of statistical results in psychology journals. Behavior Research Methods 43:666-678.

Barker, F.K. and Lutzoni, F.M. 2002. The utility of the incongruence length difference test. Systematic Biology 51:625-37.

Baum, B.R. 1992. Combining trees as a way of combining data sets for phylogenetic inference, and the desirability of combining gene trees. Taxon 41:3-10.

Beard, K. C., Marivaux, L., Chaimanee, Y. , Jaeger, J.-J., Marandat, B., Tafforeau, P., Soe, A.N., Tun, S.T. And Kyaw, A.A. 2009. A new primate from the Eocene Pondaung formation of Myanmar and the monophyly of Burmese amphipithecids. Proceedings of the Royal Society of London. Series B: Biological Sciences 276:3285-3294.

Beck, R. M. D., Godthelp, H., Weisbecker, V. Archer, M. and Hand, S.J. 2008. Australia's oldest marsupial fossils and their biogeographical implications. PLOS ONE 3:e1858+.

Beckmann, M. and von Wehrden, H. 2012. Where you search is what you get: literature 
mining - google scholar versus web of science using a data set from a literature search in vegetation science. Journal of Vegetation Science 23:1197-1199.

Bennett, S. C. 2013. The phylogenetic position of the Pterosauria within the Archosauromorpha re-examined. Historical Biology 25:545-563.

Bergsten, J. 2005 A review of long-branch attraction. Cladistics 21:163-193.

Berle, D. and Starcevic V. 2007 Inconsistencies between reported test statistics and pvalues in two psychiatry journals. International Journal of Methods in Psychiatric Research 16: 202-207.

Betancur-R, R. and Acero, A. P. 2005. Description of Cathorops mapale, a new species of sea catfish (Siluriformes: Ariidae) from the Colombian Caribbean, based on morphological and mitochondrial evidence. Zootaxa 1045:45-60.

Beutel, R. G., S.-q. Ge, and T. Hörnschemeyer 2008. On the head morphology of Tetraphalerus, the phylogeny of Archostemata and the basal branching events in Coleoptera. Cladistics 24 (3), 270-298.

Beutel, R. G., B. Wang, J.-J. Tan, S.-Q. Ge, D. Ren, and X.-K. Yang 2012. On the phylogeny and evolution of Mesozoic and extant lineages of Adephaga (Coleoptera, Insecta). Cladistics 29:147-165.

Bisconti, M. 2008. Morphology and phylogenetic relationships of a new eschrichtiid genus (Cetacea: Mysticeti) from the Early Pliocene of northern Italy. Zoo. J. Linn. Soc. 153:161-186.

Blagoderov, V. and D. Grimaldi 2004. Fossil Sciaroidea (Diptera) in Cretaceous ambers, exclusive of Cecidomyiidae, Sciaridae, and Keroplatidae. American Museum Novitates, 1-76.

Blagoderov, V., Hippa, H., and Sevcik, J. 2009. Asiorrhina, a new oriental genus of Lygistorrhinidae (Diptera: Sciaroidea) and its phylogenetic position. Zootaxa 2295:3145.

Bledsoe, A. H. and Raikow, R. J. 1990. A quantitative assessment of congruence between molecular and nonmolecular estimates of phylogeny. Journal of Molecular Evolution 30:247-259.

Bloch, J.I., Silcox, M.T. Boyer, D.M. and Sargis, E.J. 2007. New paleocene skeletons and the relationship of plesiadapiforms to crown-clade primates. Proceedings of the National Academy of Sciences of the United States of America 104:1159-1164.

Bloech, C., Weiss-Schneeweiss, H., Schneeweiss, G. M., Barfuss, M. H., Rebernig, C. A., Villaseñor, J. L. L., and Stuessy, T. F. 2009. Molecular phylogenetic analyses of nuclear and plastid DNA sequences support dysploid and polyploid chromosome number changes and reticulate evolution in the diversification of melampodium (Millerieae, Asteraceae). Molecular Phylogenetics and Evolution 53:220-233.

Bluis, J. and Shin, D.G. 2003. Nodal distance algorithm: Calculating a phylogenetic tree comparison metric. In: BIBE '03: Proceedings of the 3rd IEEE Symposium on Biolnformatics and BioEngineering. Washington, DC, USA: IEEE Computer Society. p. $87+$

Boessenecker, R. W. and Churchill, M. 2013. A reevaluation of the morphology, paleoecology, and phylogenetic relationships of the enigmatic walrus Pelagiarctos. PLOS ONE 8:e54311+. 
Bonde, N. 1976. Cladistic classification as applied to Vertebrates pp. 741-804. In: Hecht, M.K., Goody, P.C. and Hecht, B.M. (eds.) Major Patterns in Vertebrate Evolution. Plenum Press, New York.

Bouetel, V. and de Muizon, C. 2006. The anatomy and relationships of Piscobalaena nana (Cetacea, Mysticeti), a Cetotheriidae s.s. from the Early Pliocene of Peru. Geodiversitas 28:319-395.

Bourdon, E., Ricqles, A. and Cubo, J. 2009. A new transantarctic relationship: morphological evidence for a Rheidae-Dromaiidae-Casuariidae clade (Aves, Palaeognathae, Ratitae). Zoological Journal of the Linnean Society 156:641-662.

Bourque, M. 1978. Arbres de steiner et reseaux dont certains sommets sont a localisation variable. Thesis, University of Montreal.

Bravo, F. 2006. The taxonomy of Neotropical Brunettiina (Diptera, Psychodidae, Psychodinae, Mormiini), with descriptions of ten new species from Brazil and comments on the generic classification of the subtribe. Zootaxa 1134:1-28.

Bremer, B. 1996. Combined and separate analyses of morphological and molecular data in the plant family Rubiaceae. Cladistics 12:21-40.

Brix, S., Riehl, T. and Leese, F. 2011. First genetic data for species of the genus Haploniscus Richardson, 1908 (Isopoda: Asellota: Haploniscidae) from neighbouring deep-sea basins in the South Atlantic. Zootaxa 2838:79-84.

Brochu, C. A., Njau, J., Blumenschine, R. J., and Densmore, L. D. 2010. A new horned crocodile from the Plio-Pleistocene hominid sites at olduvai gorge, Tanzania. PLoS ONE 5:e9333+.

Brundin, L. 1966. Transantarctic relationships and their significance, as evidenced by chironomid midges. K. Svenska Vet. Akad. Handl. 11:1-472.

Brusatte, S. L., Benson, R. B. J., and Xu, X. 2012. A reassessment of Kelmayisaurus petrolicus, a large theropod dinosaur from the Early Cretaceous of China. Acta Palaeontologica Polonica 57:65-72.

Bryant, H. N. 1995. Why autapomorphies should be removed: A reply to Yeates. Cladistics 11:381-384.

Buckley, T. R., Simon, C., Shimodaira, H. and Chambers, G. K. 2001. Evaluating hypotheses on the origin and evolution of the New Zealand alpine cicadas (Maoricicada) using multiple-comparison tests of tree topology. Molecular Biology and Evolution 18:223-234.

Bull, J.J., Huelsenbeck, J.P., Cunningham, C.W., Swofford, D.L. and Waddell, P.J. 1993. Partitioning and combining data in phylogenetic analysis. Systematic Biology 42:384397.

Butler, R.J., Upchurch, P. and Norman, D.B. 2008. The phylogeny of the Ornithischian dinosaurs. Journal of Systematic Palaeontology 6:1-40.

California Digital Library 2013. SciVerse Hub is Retiring in August 2013. http://www.cdlib.org/cdlinfo/2013/06/17/sciverse-hub-is-retiring-in-august-2013/ [Webpage]

Cano, R. J., Poinar, H. N., Pieniazek, N. J., Acra, A., and Poinar, G. O. 1993. Amplification and sequencing of DNA from a 120-135-million-year-old weevil. Nature 363:536-538. 
Carballido, J. L., Garrido, A. C., Canudo, J. I., and Salgado, L. 2010. Redescription of Rayososaurus agrioensis Bonaparte (Sauropoda, Diplodocoidea), a rebbachisaurid from the early Late Cretaceous of Neuquén. Geobios 43:493-502.

Carbone, I., Anderson, J. B., and Kohn, L. M. (1999). Patterns of descent in clonal lineages and their multilocus fingerprints are resolved with combined gene genealogies. Evolution 16: $354-362$.

Carpenter, J.M. 1988. Choosing among multiple equally parsimonious cladograms. Cladistics 4:291-296.

Carrano, M.T. and Sampson, S.D. 2008. The phylogeny of Ceratosauria (Dinosauria: Theropoda). Journal of Systematic Palaeontology 6:183-236.

Carrasco, P. A., Mattoni, C. I., Leynaud, G. C., and Scrocchi, G. J. 2012. Morphology, phylogeny and taxonomy of South American bothropoid pitvipers (serpentes, viperidae). Zoologica Scripta 41:109-124.

Carroll, H., Ebbert, M., Clement, M. \& Snell, Q. 2007. PSODA: Better tasting and less filling than PAUP. Proceedings of the 4th Biotechnology and Bioinformatics Symposium, pp 74-78

Carroll, H., Teichert, A. R., Krein, J., Sundberg, K., Snell, Q., and Clement, M. 2009. An open source phylogenetic search and alignment package. International journal of bioinformatics research and applications 5:349-364.

Cau, A. and Fanti, F. 2011. The oldest known metriorhynchid crocodylian from the middle Jurassic of north-eastern Italy: Neptunidraco ammoniticus gen. et sp. nov. Gondwana Research 19: 550-565.

Chadegani, A. A., Salehi, H., Yunus, M. M., Farhadi, H., Fooladi, M., Farhadi, M., and Ebrahim, N. A. 2013. A comparison between two main academic literature collections: Web of Knowledge and Scopus databases. Asian Social Science 9.

Cheng, L., Chen, X., Zeng, X., and Cai, Y. 2012. A new Eosauropterygian (Diapsida: Sauropterygia) from the Middle Triassic of Luoping, Yunnan province. Journal of Earth Science 23:33-40.

Claeson, K. M., O'Leary, M. A., Roberts, E. M., Sissoko, F., Bouaré, M., Tapanila, L., Goodwin, D., and Gottfried, M. D. 2010. First mesozoic record of the stingray Myliobatis wurnoensis from Mali and a phylogenetic analysis of Myliobatidae incorporating dental characters. Acta Palaeontologica Polonica 55:655-674.

Clarke, J. A., D. T. Ksepka, N. A. Smith, and M. A. Norell 2009. Combined phylogenetic analysis of a new north american fossil species confirms widespread Eocene distribution for stem rollers (Aves, Coracii). Zoological Journal of the Linnean Society 157 (3), 586-611.

Clarke, D. J. 2011. Testing the phylogenetic utility of morphological character systems, with a revision of Creophilus Leach (Coleoptera: Staphylinidae). Zoological Journal of the Linnean Society 163:723-812.

Clennett, J. C. B., Chase, M. W., Forest, F., Maurin, O., and Wilkin, P. 2012. Phylogenetic systematics of erythronium (liliaceae): morphological and molecular analyses. Botanical Journal of Linnean Society 170:504-528.

Cobbett A., Wilkinson, M. and Wills, M.A. 2007. Fossils impact as hard as living taxa in parsimony analyses of morphology. Systematic Biology 56:753-766. 
Collard, M., Gibbs, S. and Wood, B. 2001. Phylogenetic utility of higher primate postcranial morphology. American Journal of Physical Anthropology Supplement 32: 52.

Colless, D.H. 1980. Congruence between morphometric and allozyme data for Menidia species: A reappraisal. Systematic Zoology 29:288-299.

Conrad, J. L. 2008. Phylogeny and systematics of Squamata (Reptilia) based on morphology. Bulletin of the American Museum of Natural History 310:1-182.

Conway Morris, S. 1977. A new metazoan from the Cambrian Burgess Shale of British Columbia. Palaeontology 20:623-640.

Csiki, Z., Vremir, M., Brusatte, S. L., and Norell, M. A. 2010. An aberrant island-dwelling theropod dinosaur from the Late Cretaceous of Romania. Proceedings of the National Academy of Sciences 107:15357-15361.

Cunningham, C. W. 1997a Can three incongruence tests predict when data should be combined? Molecular Biology and Evolution 14(7):733-740.

Cunningham, C. W. 1997b Is congruence between data partitions a reliable predictor of phylogenetic accuracy? Empirically testing an iterative procedure for choosing among phylogenetic methods. Systematic Biology 46: 464-478.

D’Erchia, A.M., Gissi, C., Pesole, G., Saccone, C.and Arnasonn, U. 1996. The guinea-pig is not a rodent. Nature 381:597-600.

Davis, M. P. 2010. Evolutionary relationships of the Aulopiformes (Euteleostei: Cyclosquamata): a molecular and total evidence approach pp. 431-470. In: J.S. Nelson, H.P. Schultze and M.V.H. Wilson (eds.) Origin and Phylogenetic Interrelationships of Teleosts. Verlag Dr. Friedrich Pfeil, Munchen, Germany.

Daza, J. D., Alifanov, V. R., and Bauer, A. M. 2012. A redescription and phylogenetic reinterpretation of the fossil lizard Hoburogekko suchanovi Alifanov, 1989 (Squamata, Gekkota), from the Early Cretaceous of Mongolia. Journal of Vertebrate Paleontology 32:1303-1312.

De Pietri, V. L., Costeur, L., Güntert, M., and Mayr, G. 2011. A revision of the Lari (Aves, Charadriiformes) from the Early Miocene of Saint-Gérand-le-Puy (Allier, France). Journal of Vertebrate Paleontology 31:812-828.

de Vienne, D.M., Giraud, T. and Martin, O.C. 2007. A congruence index for testing topological similarity between trees. Bioinformatics 23:3119-3124.

Degnan, J.H. and Rosenberg, N.A. 2009. Gene tree discordance, phylogenetic inference and the multispecies coalescent. Trends in Ecology and Evolution 24:332-340.

Deng, T. 2008. A new elasmothere (Perissodactyla, Rhinocerotidae) from the Late Miocene of the Linxia basin in Gansu, China. Geobios 41:719-728.

Deo, A. J., Costa, R., DeLisi, L. E., DeSalle, R., and Haghighi, F. 2010. A novel analytical framework for dissecting the genetic architecture of behavioral symptoms in neuropsychiatric disorders. PLoS ONE 5:e9714+.

Dietze, K. 2009. Morphology and phylogenetic relationships of certain neoteleostean fishes from the Upper Cretaceous of Sendenhorst, Germany. Cretaceous Research 30:559-574.

Dilernia, D. A., Jones, L., Rodriguez, S., Turk, G., Rubio, A. E., Pampuro, S., GomezCarrillo, M., Bautista, C., Deluchi, G., Benetucci, J., Lasala, M. B., Lourtau, L., Losso, 
M. H., Perez, H., Cahn, P., and Salomón, H. 2008. HLA-driven convergence of HIV-1 viral subtypes $b$ and $f$ toward the adaptation to immune responses in human populations. PLoS ONE 3:e3429+.

Diogo, R. 2004. Morphological Evolution, Adaptations, Homoplasies, Constraints, and Evolutionary Trends: Catfishes as a Case Study on General Phylogeny \& Macroevolution. Science Publishers, Enfield.

Diogo, R. 2007. On the origin and evolution of higher-clades: osteology, myology, phylogeny and macroevolution of bony fishes and the rise of tetrapods. Enfield, Science Publishers.

Diogo, R., Doadrio, I., and Vandewalle, P. 2008. Teleostean phylogeny based on osteological and myological characters. International Journal of Morphology 26:463522.

Dockes, J-F. 2013. Recoll. http://www.lesbonscomptes.com/recoll/ [accessed 2013-06-25]

Dolphin, K., Belshaw, R., Orme, C.D.L. and Quicke, D.L.J. 2000. Noise and incongruence: Interpreting results of the incongruence length difference test. Molecular Phylogenetics and Evolution 17:401-406.

Dong, X.-P. P., Donoghue, P. C., Cunningham, J. A., Liu, J.-B. B., and Cheng, H. 2005. The anatomy, affinity, and phylogenetic significance of Markuelia. Evolution \& Development 7:468-482.

Donoghue, M. J., Doyle, J. A., Gauthier, J., Kluge, A. G., and Rowe, T. 1989. The importance of fossils in phylogeny reconstruction. Annual Review of Ecology and Systematics 20:431-460.

Donoghue, M.J. and Ree, R.H. 2000. Homoplasy and developmental constraint: A model and an example from plants. American Zoologist 40:759-769.

Donoghue, P. C. J. and Benton, M. J. 2007. Rocks and clocks: calibrating the Tree of Life using fossils and molecules. Trends in Ecology and Evolution 22:424-431.

Doolittle, W.F. and Bapteste, E. 2007. Pattern pluralism and the tree of life hypothesis. Proceedings of the National Academy of Sciences of the United States of America 104:2043-2049.

Dowton, M. and Austin, A. D. 2002. Increased congruence does not necessarily indicate increased phylogenetic accuracy-the behavior of the incongruence length difference test in mixed-model analyses. Systematic Biology 51:19-31.

Draper, I., Hedenas, L. and Grimm, G. L. 2007. Molecular and morphological incongruence in European species of Isothecium (Bryophyta). Molecular Phylogenetics and Evolution 42:700-716.

Drew, B. T., Gazis, R., Cabezas, P., Swithers, K. S., Deng, J., Rodriguez, R., Katz, L. A., Crandall, K. A., Hibbett, D. S., and Soltis, D. E. 2013. Lost branches on the tree of life. PLoS Biology 11:e1001636+.

Eddy, D. R. and Clarke, J. A. 2011. New information on the cranial anatomy of Acrocanthosaurus atokensis and its implications for the phylogeny of Allosauroidea (Dinosauria: Theropoda). PLoS ONE 6:e17932+. [PMCID: PMC3061882]

Edgecombe, G. D. and Hollington, L. M. 2005. Morphology and relationships of a new species of Henicops (Chilopoda: Lithobiomorpha) from New South Wales and Queensland, Australia. Zootaxa 961:1-20. 
Edgecombe, G. D. 2010. Palaeomorphology: fossils and the inference of cladistic relationships. Acta Zoologica 91:72-80.

Eernisse, D. J. and Kluge, A. G. 1993. Taxonomic congruence versus total evidence, and amniote phylogeny inferred from fossils, molecules, and morphology. Molecular Biology and Evolution 10:1170-1195.

Egan, M.G. 2006 Support versus corroboration. Journal of Biomedical Informatics 39:7285.

Eichholz, 1949. Aristotle's Theory of the Formation of Metals and Minerals. The Classical Quarterly 43:141-146.

Erwin, D. H. 2008. Extinction as the loss of evolutionary history. Proceedings of the National Academy of Sciences of the United States of America 105 Suppl 1:1152011527.

Estabrook, G.F. 1968. A general solution in partial orders for the Camin-Sokal model in phylogeny. Journal of Theoretical Biology 21:421-438.

Estabrook, G.F., McMorris, F.R. and Meacham, C.A. 1985. Comparison of undirected trees based on subtrees of four evolutionary units. Systematic Zoology 34:193-200.

Ezcurra, M.D. and Cuny, G. 2007. The coelophysoid Lophostropheus airelensis, gen. nov.: A review of the systematics of "Liliensternus" airelensis from the Triassic-Jurassic outcrops of Normandy (France). Journal of Vertebrate Paleontology 27:73+.

Fairbairn, D. J. 2011. The advent of mandatory data archiving. Evolution 65:1-2.

Faith, D.P. and Cranston, P.S. 1991. Could a cladogram this short have arisen by chance alone?: On permutation tests for cladistic structure. Cladistics 7:1-28.

Faivovich, J. 2002. A cladistic analysis of Scinax (Anura: Hylidae). Cladistics 18:367-393.

Farke, A. A., Ryan, M. J., Barrett, P. M., Tanke, D. H., Braman, D. R., Loewen, M. A., and Graham, M. R. 2011. A new centrosaurine from the late cretaceous of alberta, canada, and the evolution of parietal ornamentation in horned dinosaurs. Acta Palaeontologica Polonica 56:691-702.

Farris, J.S. 1971. The hypothesis of nonspecificity and taxonomic congruence. Annual Review of Ecology and Systematics 2:277-302.

Farris, J.S. 1973. On comparing the shapes of taxonomic trees. Systematic Zoology 22:50-54.

Farris, J. S. 1976. Phylogenetic classification of fossils with recent species. Systematic Zoology 25:271-282.

Farris, J. S. 1989a. The retention index and the rescaled consistency index. Cladistics 5: 417-419.

Farris, J.S. 1989b. The retention index and homoplasy excess. Systematic Biology 38:406-407.

Farris, J.S. 1991. Excess Homoplasy Ratios. Cladistics 7: 81-91.

Farris, J. S. 1997. Combinability and congruence. Cladistics 13, 170. [Conference Abstract]

Farris, J.S., Kluge, A.G. and Eckardt, M.J. 1970. A numerical approach to phylogenetic systematics. Systematic Zoology 19:172-189. 
Farris, J.S., Källersjö, M., Kluge, A.G. and Bult, C. 1995a. Constructing a significance test for incongruence. Systematic Biology 44:570-572.

Farris, J.S., Källersjö, M., Kluge, A.G. and Bult, C. 1995b. Testing significance of incongruence. Cladistics 10:315-319.

Felsenstein, L. 1985. Confidence Limits on Phylogenies: An Approach Using the Bootstrap. Evolution 39:783-791.

Felsenstein, J. 1988. Phylogenies from molecular sequences: inference and reliability. Annual Review of Genetics 22:521-565.

Fidler, F., Burgman, M. A., Cumming, G., Buttrose, R., and Thomason, N. 2006. Impact of criticism of null-hypothesis significance testing on statistical reporting practices in conservation biology. Conservation Biology 20:1539-1544.

Fikáček, M., Prokin, A., Angus, R. B., Ponomarenko, A., Yue, Y., Ren, D., and Prokop, J. 2012. Phylogeny and the fossil record of the helophoridae reveal jurassic origin of extant hydrophiloid lineages (Coleoptera: Polyphaga). Systematic Entomology 37:420447.

Finarelli, J. 2008. A total evidence phylogeny of the Arctoidea (Carnivora: Mammalia): Relationships among basal taxa. Journal of Mammalian Evolution 15:231-259.

Finarelli, J.A. and Clyde, W.C. 2004. Reassessing hominoid phylogeny: Evaluating congruence in the morphological and temporal data. Paleobiology 30: 614-651.

Fingerman, S. 2004. Elsevier Holds Official Launch Events for Scopus. http://newsbreaks.infotoday.com/NewsBreaks/Elsevier-Holds-Official-Launch-Eventsfor-Scopus-16330.asp

Fisher-Reid, C. and Wiens, J. 2011. What are the consequences of combining nuclear and mitochondrial data for phylogenetic analysis? lessons from plethodon salamanders and 13 other vertebrate clades. BMC Evolutionary Biology 11:300+.

Fitzgerald, E.M.G. 2010. The morphology and systematics of Mammalodon colliveri (Cetacea: Mysticeti), a toothed mysticete from the Oligocene of Australia. Zoological Journal of the Linnean Society 158: 367-476.

Ford, L. and O'Hara, L. H. 2008. It's all academic: Google scholar, scirus, and windows live academic search. Journal of Library Administration 46:43-52.

Fordyce, R. E. and F. G. Marx 2013. The pygmy right whale Caperea marginata: the last of the cetotheres. Proceedings of the Royal Society B: Biological Sciences 280 (1753)

Forey, P. L. and Fortey, R. A. 2001. Fossils in the reconstruction of phylogeny. Pp. 515519 in D. E. G. Briggs \& P. R. Crowther (editors), Palaeobiology II. Blackwell Science, Inc., Malden, Massachusetts.

Forey, P. L. 2004. Systematics and Paleontology. In: D. M. Williams and P. L. Forey (eds.) Milestones in Systematics. Systematics Association Special Volume, CRC Press, 149180.

Forey, P.L. 2005. Cladistics For Palaeontologists. Palaeontology Newsletter http://www.palass.org/modules.php?name=palaeo\&page=19\&sec=newsletter

Frazzetta, T. H. 2012. Flatfishes, turtles, and bolyerine snakes: Evolution by small steps or large, or both? Evolutionary Biology 39: 30-60.

Friedman, M. 2007. Styloichthys as the oldest coelacanth: Implications for early 
osteichthyan interrelationships. Journal of Systematic Palaeontology 5:289-343.

Friedman, M. 2008. The evolutionary origin of flatfish asymmetry. Nature 454:209-212.

Fritz, U., Branch, W. R., Hofmeyr, M. D., Maran, J., Prokop, H., Schleicher, A., Iroký, Stuckas, H., Vargas-Ramírez, M., Vences, M., and Hundsdörfer, A. K. 2011. Molecular phylogeny of african hinged and helmeted terrapins (testudines: Pelomedusidae: Pelusios and pelomedusa). Zoologica Scripta 40:115-125.

Frobisch, J. 2007. The cranial anatomy of Kombuisia frerensis Hotton (Synapsida, Dicynodontia) and a new phylogeny of anomodont therapsids. Zoological Journal of the Linnean Society 150:117-144.

Fu, J. and Murphy, R.W. 1999 Discriminating and locating character covariance: an application of permutation tail probability (PTP) analyses. Systematic Biology 48: 380395.

Gaffney, E.S., Hooks, G.E. and Schneider, V.P. 2009. New material of North American side-necked turtles (Pleurodira: Bothremydidae). American Museum Novitates 3655:126.

Gaffney, E. S. and Krause, D. W. 2011. Sokatra, a new Side-Necked turtle (Late Cretaceous, Madagascar) and the diversification of the main groups of pelomedusoides. American Museum Novitates 1-28.

Garcia-Perez, M. 2012. Reviewer's report of: Gehanno et al (2013) Is Google Scholar sensitive enough to be used alone for systematic reviews? http://www.biomedcentral.com/imedia/8023217788144832_comment.pdf

Garwood, R. J., Dunlop, J. A., Giribet, G., and Sutton, M. D. 2011. Anatomically modern carboniferous harvestmen demonstrate early cladogenesis and stasis in opiliones. Nature Communications 2:444+.

Gates, T.A. and Sampson, S.D. 2007. A new species of Gryposaurus (Dinosauria: Hadrosauridae) from the late Campanian Kaiparowits formation, Southern Utah, USA. Zoological Journal of the Linnean Society 151:351-376.

Gaubert, P., Wozencraft, W.C., Cordeiro-Estrela, P. and Veron, G. 2005. Mosaics of convergences and noise in morphological phylogenies: What's in a viverrid-like carnivoran? Systematic Biology 54:865-894.

Gaudin, T., Emry, R. and Wible, J. 2009. The phylogeny of living and extinct pangolins (Mammalia, Pholidota) and associated taxa: A morphology based analysis. Journal of Mammalian Evolution 16:235-305.

Gauthier, J., Kluge, A. G., and Rowe, T. 1988. Amniote phylogeny and the importance of fossils. Cladistics 4:105-209.

Gehanno, J. F., Rollin, L., and Darmoni, S. 2013. Is the coverage of google scholar enough to be used alone for systematic reviews? BMC Medical Informatics and Decision Making 13:7+.

Gilbert, C. C. 2013. Cladistic analysis of extant and fossil african papionins using craniodental data. Journal of Human Evolution 64:399-433.

Giribet, G. 2010. A new dimension in combining data? the use of morphology and phylogenomic data in metazoan systematics. Acta Zoologica 91:11-19.

Giustini, D. 2013. Is Google scholar enough for SR searching? No. 
http://blogs.ubc.ca/dean/2013/01/is-google-scholar-enough-for-sr-searching-no/ [Blog post]

Goddard, W., Kubicka, E., Kubicki, G. and McMorris, F.R. 1994. The agreement metric for labelled binary trees. Mathematical Biosciences 123:215-226.

Godefroit, P., Shulin, H., Tingxiang, Y. and Lauters, P. 2008. New hadrosaurid dinosaurs from the uppermost Cretaceous of Northeastern China. Acta Palaeontologica Polonica 53:47-74.

Goldman, N., Anderson, J.P. and Rodrigo, A.G. 2000. Likelihood-based tests of topologies in phylogenetics. Systematic Biology 49:652-670.

Goloboff P. A. 1999a. Analyzing large data sets in reasonable times: Solutions for composite optima. Cladistics 15:415-428.

Goloboff, P.A. 1999b. NONA (NO NAME) ver. 2 Published by the author, Tucumán, Argentina.

Goloboff, P. A., Farris, J. S., and Nixon, K. C. 2008. TNT, a free program for phylogenetic analysis. Cladistics 24:774-786.

Goloboff, P.A. 2008. Calculating SPR distances between trees. Cladistics 24:591-597.

Gonzalez-Riga, B., Previtera, E. and Pirrone, C. 2009. Malarguesaurus florenciae gen. et sp. nov., a new titanosauriform (Dinosauria, Sauropoda) from the Upper Cretaceous of Mendoza, Argentina. Cretaceous Research 30:135-148.

Google 2013. Our history in depth. http://www.google.com/about/company/history/

Gould, G.C. 2001. The phylogenetic resolving power of discrete dental morphology among extant hedgehogs and the implications for their fossil record. American Museum Novitates 3340:1-52.

Graham, D. 2013 Academic Publishing: Survey of funders supports the benign Open Access outcome priced into shares. HSBC Global Research 1-36

Grant, T., and Kluge, A.G. 2003. Data exploration in phylogenetic inference: scientific, heuristic, or neither. Cladistics 19: 379-418.

Grantham, T. 2004. The role of fossils in phylogeny reconstruction: Why is it so difficult to integrate paleobiological and neontological evolutionary biology? Biology and Philosophy 19:687-720.

Greenwood, D. R., Hill, C. R., and Conran, J. G. 2013. Prumnopitys anglica sp. nov. (Podocarpaceae) from the Eocene of England. Taxon 62(3) 565-580.

Guang-Hui, X. U. and Mee-Mann Chan, G. 2009. Redescription of Paralycoptera wui Chang \& Chou, 1977 (Teleostei: Osteoglossoidei) from the Early Cretaceous of eastern China. Zoo. J. of the Linn. Soc. 157:83-106.

Guayasamin, J. M., Castroviejo-Fisher, S., Trueb, L., Ayarzaguena, J., Rada, M., Vila, C. Phylogenetic systematics of Glassfrogs (Amphibia: Centrolenidae) and their sister taxon Allophryne ruthveni. Zootaxa 2100:1-97.

Gutiérrez, G. and Marín, A. 1998. The most ancient DNA recovered from an amberpreserved specimen may not be as ancient as it seems. Molecular Biology and Evolution 15:926-929.

Harmer, A. M. T. and Framenau, V. W. 2008. Telaprocera, a new Australian orb-web spider 
genus with elongated webs (Araneae: Araneidae). Zootaxa 1956, 59-80.

Hastings, A.K., Bloch, J.I. and Jaramillo, C.A. 2011. A new longirostrine dyrosaurid (Crocodylomorpha, Mesoeucrocodylia) from the Paleocene of north-eastern Colombia: Biogeographic and behavioural implications for New-World Dyrosauridae. Palaeontology 54:1095-1116.

Hebsgaard, M. B., Phillips, M. J., and Willerslev, E. 2005. Geologically ancient DNA: fact or artefact? Trends in Microbiology 13:212-220.

Hedges, S.B. and Sibley, C.G. 1994. Molecules vs. morphology in avian evolution: the case of the "pelecaniform" birds. Proceedings of the National Academy of Sciences of the United States of America 91:9861-9865.

Hedges, S.B. and Maxson, L.R. 1996. Re: Molecules and morphology in amniote phylogeny. Molecular Phylogenetics and Evolution 6:312-314.

Hennig, W. 1950. Grundzüge einer Theorie der phylogenetischen Systematik, Berlin: Deutscher Zentralverlag

Hennig, W. 1966. Phylogenetic Systematics. University of Illinois Press, Urbana.

Hill, R.V. 2005. Integration of morphological data sets for phylogenetic analysis of Amniota: The importance of integumentary characters and increased taxonomic sampling. Systematic Biology 54:530-547.

Hillis, D. M. 1996. Inferring complex phylogenies. Nature 383:140-141.

Hillis, D. M. 1998. Taxonomic sampling, phylogenetic accuracy, and investigator bias. Systematic Biology 47:3-8.

Hilton, E. J. and P.L. Forey. 2009. Redescription of ?Chondrosteus acipenseroides Egerton, 1858 (Acipenseriformes, ?Chondrosteidae) from the lower Lias of Lyme Regis (Dorset, England), with comments on the early evolution of sturgeons and paddlefishes. Journal of Systematic Palaeontology 7:427-453.

Hinchliff, C. E. and Roalson, E. H. 2013. Using supermatrices for phylogenetic inquiry: an example using the sedges. Systematic Biology 62:205-219.

Hipp, A.L., Hall, J.C. and Sytsma, K.J. 2004. Congruence versus phylogenetic accuracy: Revisiting the incongruence length difference test. Systematic Biology 53:81-89.

Holland, T. and Long, J.A. 2009. On the phylogenetic position of Gogonasus andrewsae, within the Tetrapodomorpha. Acta Zoologica 90: 285-296.

Hoot, S. B., Magallon, S., and Crane, P. R. (1999). Phylogeny of basal eudicots based on three molecular data sets: atpB, rbcL and $18 \mathrm{~S}$ nuclear ribosomal DNA sequences. Annals of the Missouri Botanical Garden 86: 1-32.

Hospitaleche, C.A., Tambussi, C., Donato, M. and Cozzuol, M. 2007. A new Miocene penguin from Patagonia and its phylogenetic relationships. Acta Palaeontologica Polonica 52:299-314.

Hoyal Cuthill, J. F., Braddy, S. J., and Donoghue, P. C. J. 2010. A formula for maximum possible steps in multistate characters: isolating matrix parameter effects on measures of evolutionary convergence. Cladistics 26:98-102.

Huelsenbeck, J. P. 1991. When are fossils better than extant taxa in phylogenetic analysis? Systematic Zoology 40:458-469. 
Hull, D.L. 1990. Science as a Process: An Evolutionary Account of the Social and Conceptual Development of Science. University of Chicago Press, Chicago.

Hunt, G. 2008. Gradual or pulsed evolution: When should punctuational explanations be preferred? Paleobiology 34: 360-377.

Hurley, I.A., Mueller, R.L., Dunn, K.A., Schmidt, E.J., Friedman, M., Ho, R.K., Prince, V.E., Yang, Z., Thomas, M.G. and Coates, M.I. 2007. A new time-scale for ray-finned fish evolution. Proceedings of the Royal Society of London. Series B: Biological Sciences 274:489-498.

Huson, D.H. and Scornavacca, C. 2012. Dendroscope 3: An interactive tool for rooted phylogenetic trees and networks. Systematic Biology 61:1061-1067.

Hutchinson, M. N., Skinner, A., and Lee, M. S. Y. 2012. Tikiguania and the antiquity of squamate reptiles (lizards and snakes). Biology Letters 8:665-669.

Imamura, H., Shirai, S.M. and Yabe, M. 2005. Phylogenetic position of the family Trichodontidae (Teleostei: Perciformes), with a revised classification of the perciform suborder Cottoidei. Ichthyological Research 52:264-274.

Janssens, S. B., Wilson, Y. S., Yuan, Y.-M., Nagels, A., Smets, E. F., and Huysmans, S. 2012. A total evidence approach using palynological characters to infer the complex evolutionary history of the asian Impatiens (Balsaminaceae). Taxon 61:355-367.

Jenner, R. A., Dhubhghaill, C. N., Ferla, M. P., and Wills, M. A. 2009. Eumalacostracan phylogeny and total evidence: limitations of the usual suspects. BMC Evolutionary Biology 9:21+.

Jenner, R. A. 2004a. Accepting partnership by submission? morphological phylogenetics in a molecular millennium. Systematic Biology 53:333-359.

Jenner, R. A. 2004b. When molecules and morphology clash: reconciling conflicting phylogenies of the metazoa by considering secondary character loss. Evolution \& Development 6:372-378+.

Ji, Q., Z.-X. Luo and Ji, S.-A. 1999. A Chinese triconodon mammal and mosaic evolution of the mammalian skeleton. Nature 398: 326-330.

Jinha, A. E. 2010. Article 50 million: an estimate of the number of scholarly articles in existence. Learned Publishing 23:258-263.

Johnson, K. P. and Sorensen, M. D. 1998. Comparing molecular evolution in two mitochondrial protein coding genes (cytochrome $b$ and ND2) in the dabbling ducks (Tribe: Anatini). Molecular Phylogenetics and Evolution 10: 82-94.

Jouve, S., larochene, M., Bouya, B. and Amaghzaz, M. 2006. A new species of Dyrosaurus (Crocodylomorpha, Dyrosauridae) from the early Eocene of Morocco: Phylogenetic implications. Zoological Journal of the Linnean Society 148:603-656.

Joyce, W.G. 2007. A phylogeny of Mesozoic turtles. Bull. of the Peabody Museum of Natural History 48:3-102

Joyce, W. G. and Sterli, J. 2012. Congruence, non-homology, and the phylogeny of basal turtles. Acta Zoologica 93:149-159.

Karasawa, H., Schweitzer, C.E., 2006. A new classification of the Xanthoidea sensu lato (Crustacea: Decapoda: Brachyura) based on phylogenetic analysis and traditional systematics and evaluation of all fossil Xanthoidea sensu lato. Contr. Zool. 75:23-73. 
Karasawa, H., Schweitzer, C.E., Feldmann, R.M. 2011. Phylogenetic analysis and revised classification of podotrematous Brachyura (Decapoda) including extinct and extant families. J. Crust. Biol. 31:523-565.

Kempthorne, O. 1952. Design and Analysis of Experiments. Wiley, New York.

Kerr, P. H. (2010) Phylogeny and classification of Rhagionidae, with implications for

Tabanomorpha (Diptera: Brachycera). Zootaxa 2592:1-133.

Kishino, H. and Hasegawa, M. 1989. Evaluation of the maximum likelihood estimate of the evolutionary tree topologies from DNA sequence data, and the branching order in hominoidea. Journal of Molecular Evolution 29:170-179.

Kitching, I., Forey, P., Humphries, C. and Williams, D. 1998. Cladistics: Theory and Practice of Parsimony Analysis (Systematics Association Special Volume). Oxford University Press, Oxford.

Klassen, G. J., Mooi, R.D. and Locke, A. 1991. Consistency indices and random data. Systematic Zoology 40: 446-457.

Klingenberg, C.P. 2008. Morphological integration and developmental modularity. Annual Reviews of Ecology, Evolution and Systematics 39:115-132.

Klug, S. 2010. Monophyly, phylogeny and systematic position of the Synechodontiformes (Chondrichthyes, Neoselachii). Zoologica Scripta 39:37-49.

Kluge, A. G. and Farris, J. S. 1969. Quantitative phyletics and the evolution of anurans. Systematic Zoology 18:1-32.

Kluge, A.G. 1989. A concern for evidence and a phylogenetic hypothesis of relationships among Epicrates (Boidae, Serpentes). Systematic Zoology 38:7-25.

Klymiuk, A. A. and Stockey, R. A. 2012. A Lower Cretaceous (Valanginian) seed cone provides the earliest fossil record for Picea (Pinaceae). American Journal of Botany 99:1069-1082.

Koperwas J., Walczak K. 2011. Tree edit distance for leaf-labelled trees on free leafset and its comparison with frequent subsplit dissimilarity and popular distance measures. BMC Bioinformatics 12:204+.

Koski, K. 2007. The mandibular complex. European Journal of Orthodontics 29: i118-i123.

Kroh, A. and Smith, A. B. 2010. The phylogeny and classification of post-Palaeozoic echinoids. Journal of Systematic Palaeontology 8:147-212.

Ksepka, D.T. 2009. Broken gears in the avian molecular clock: New phylogenetic analyses support stem galliform status for Gallinuloides wyomingensis and rallid affinities for Amitabha urbsinterdictensis. Cladistics 25:173-197.

Ksepka, D. T. and Clarke, J. A. 2010. New fossil mousebird (Aves: Coliiformes) with feather preservation provides insight into the ecological diversity of an Eocene north american avifauna. Zoological Journal of the Linnean Society 160:685-706.

Ksepka, D. T., J. A. Clarke, S. J. Nesbitt, F. B. Kulp, and L. Grande 2013. Fossil evidence of wing shape in a stem relative of swifts and hummingbirds (aves, Pan-Apodiformes). Proceedings of the Royal Society B: Biological Sciences 280:20130580+.

Kumar, S., Skjaeveland, A., Orr, R., Enger, P., Ruden, T., Mevik, B.H., Burki, F., Botnen, A. and Tabrizi, K.S. 2009. AIR: A batch-oriented web program package for construction of 
supermatrices ready for phylogenomic analyses. BMC Bioinformatics 10:357+.

Kurochkin, E. N. 1996. A new enantiornithid of the Mongolian Late Cretaceous, and a general appraisal of the Infraclass Enantiornithes (Aves). Russian Academy of Sciences 60pp.

Lambert, O. 2008. A new porpoise (Cetacea, Odontoceti, Phocoenidae) from the Pliocene of the North Sea. Journal of Vertebrate Paleontology 28:863-872.

Lambert, O., de Muizon, C., and Bianucci, G. 2013. The most basal beaked whale Ninoziphius platyrostris Muizon, 1983: clues on the evolutionary history of the family Ziphiidae (Cetacea: Odontoceti). Zool J Linn Soc 167:569-598.

Langille, M. G. I. and Eisen, J. A. 2010. BioTorrents: A file sharing service for scientific data. PLOS ONE 5:e10071+

Le Quesne, W.J. 1969. A method of selection of characters in numerical taxonomy. Systematic Biology 18:201-205.

Lee, M.S.Y. 2001a. Molecules, morphology, and the monophyly of diapsid reptiles. Contributions to Zoology 70:1-22.

Lee, M.S.Y. 2001b. Uninformative characters and apparent conflict between molecules and morphology. Molecular Biology and Evolution 18:676-680.

Lee, M.S.Y. and Scanlon, J. D. 2002. Snake phylogeny based on osteology, soft anatomy and ecology. Biological Reviews 77:333-401.

Leebens-Mack, J., Vision, T., Brenner, E., Bowers, J. E., Cannon, S., Clement, M. J., Cunningham, C. W., dePamphilis, C., deSalle, R., Doyle, J. J., Eisen, J. A., Gu, X., Harshman, J., Jansen, R. K., Kellogg, E. A., Koonin, E. V., Mishler, B. D., Philippe, H., Pires, J. C., Qiu, Y.-L. L., Rhee, S. Y., Sjölander, K., Soltis, D. E., Soltis, P. S., Stevenson, D. W., Wall, K., Warnow, T., and Zmasek, C. 2006. Taking the first steps towards a standard for reporting on phylogenies: Minimum information about a phylogenetic analysis (MIAPA). Omics : a journal of integrative biology 10:231-237.

Legg, D. A., Ma, X., Wolfe, J. M., Ortega-Hernandez, J., Edgecombe, G. D., and Sutton, M. D. 2011. Lobopodian phylogeny reanalysed. Nature 476:E1.

Legg, D. A., Sutton, M. D., Edgecombe, G. D., and Caron, J.-B. 2012. Cambrian bivalved arthropod reveals origin of arthrodization. Proceedings of the Royal Society B: Biological Sciences 279:4699-4704.

Lehtonen, S., Sääksjärvi, I. E., Ruokolainen, K., and Tuomisto, H. 2011. Who is the closest extant cousin of humans? total-evidence approach to hominid phylogenetics via simultaneous optimization. Journal of Biogeography 38:805-808.

Leigh, J.W., Lapointe, F.J., Lopez, P. and Bapteste, E. 2011. Evaluating phylogenetic congruence in the post-genomic era. Genome Biology and Evolution 3:571-587.

Leighton, L.R. and Maples, C.G. 2002. Evaluating internal versus external characters: Phylogenetic analyses of the Echinoconchidae, Buxtoniinae, and Juresaniinae (Phylum Brachiopoda). Journal of Paleontology 76:659-671.

Li, C., Lee, J.S. Groebner, J.L et al. 2005.

Li, P.-P., Gao, K.-Q., Hou, L.-H. and Xu, X. 2007. A gliding lizard from the early Cretaceous of China. Proceedings of the National Academy of Sciences of the United States of America 104:5507-5509. 
Lin, Y., Rajan, V. and Moret, B. 2011. A metric for phylogenetic trees based on matching bioinformatics research and applications. Lecture Notes in Computer Science 6674:197-208

Linnaeus, C. 1745. Gothländska Resa på Riksens Högloflige Ständers Befallningf förrättad åhr 1741. Stockholm \& Uppsala. [in Swedish]

Linnaeus, C. 1758. Systema Naturae, Ed. 10, Vol. 1. 824 pp. Salvii, Holmiae.

Lister, A. M., Edwards, C.J., Nock, D.A.W., Bunce, M., van Pijlen, I.A., Bradley, D.G., Thomas, M.G. and Barnes, I. 2005. The phylogenetic position of the 'giant deer' Megaloceros giganteus. Nature 438:850-853.

Liu, J., Steiner, M., Dunlop, J. A., Keupp, H., Shu, D., Ou, Q., Han, J., Zhang, Z., and Zhang, X. 2011a. An armoured cambrian lobopodian from China with arthropod-like appendages. Nature 470:526-530.

Liu, J., Steiner, M., Dunlop, J. A., Keupp, H., Shu, D., Ou, Q., Han, J., Zhang, Z., and Zhang, X. 2011b. Liu et al. reply. Nature 476:E1.

Lloyd, G. T. 2009. Matrices http://www.graemetlloyd.com/matr.html

Lopardo, L., Giribet, G., and Hormiga, G. 2011. Morphology to the rescue: molecular data and the signal of morphological characters in combined phylogenetic analyses-a case study from mysmenid spiders (Araneae, Mysmenidae), with comments on the evolution of web architecture. Cladistics 27:278-330.

Lopez, P. and Bapteste, E. 2009. Molecular phylogeny: reconstructing the forest. Comptes Rendus Biologies 332:171-182.

López-Antoñanzas, R., Flynn, L. J., and Knoll, F. 2013. A comprehensive phylogeny of extinct and extant rhizomyinae (Rodentia): evidence for multiple intercontinental dispersals. Cladistics 29:247-273.

Lord, E., Leclercq, M., Boc, A., Diallo, A. B., and Makarenkov, V. 2012. Armadillo 1.1: An original workflow platform for designing and conducting phylogenetic analysis and simulations. PLOS ONE 7:e29903+.

Lu, J., Unwin, D.M., Jin, X., Liu, Y. and Ji, Q. 2010. Evidence for modular evolution in a long-tailed pterosaur with a pterodactyloid skull. Proceedings of the Royal Society of London. Series B: Biological Sciences 277:383-389.

Luo, Z.-X. X., Yuan, C.-X. X., Meng, Q.-J. J., and Ji, Q. 2011. A jurassic eutherian mammal and divergence of marsupials and placentals. Nature 476:442-445.

Lutzoni, F.M. 1997. Phylogeny of lichen- and non-lichen-forming omphalinoid mushrooms and the utility of testing for combinability among multiple data sets. Systematic Biology 46:373-406.

Lyson, T.R. and Joyce, W.G. 2009. A new species of Palatobaena (Testudines: Baenidae) and a maximum parsimony and Bayesian phylogenetic analysis of Baenidae. Journal of Paleontology 83:457-470.

Maddison, W. P. 1997. Gene trees in species trees. Systematic Biology 46:523-536.

Maddison, D. R., Schulz, K-S., and Maddison, W. P. The Tree of Life Web Project. Zootaxa 1668:19-40.

Manegold, A. and Töpfer, T. 2012. The systematic position of hemicircus and the stepwise evolution of adaptations for drilling, tapping and climbing up in true woodpeckers 
(picinae, picidae). Journal of Zoological Systematics and Evolutionary Research

Manos, P. S., Soltis, P. S., Soltis, D. E., Manchester, S. R., Oh, S.-H., Bell, C. D., Dilcher, D. L., and Stone, D. E. 2007. Phylogeny of extant and fossil Juglandaceae inferred from the integration of molecular and morphological data sets. Systematic Biology 56:412430.

Mariadassou, M., Bar-Hen, A., and Kishino, H. 2012. Taxon influence index: assessing taxon-induced incongruities in phylogenetic inference. Systematic Biology 61:337-345.

Martinelli, A.G. and Rougier, G.W. 2007. On Chaliminia musteloides (Eucynodontia: Tritheledontidae) from the Late Triassic of Argentina, and a phylogeny of Ictidosauria. Journal of Vertebrate Paleontology 27:442-460.

Martinez, R.N. and Alcober, O.A. 2009. A basal sauropodomorph (Dinosauria: Saurischia) from the Ischigualasto formation (Triassic, Carnian) and the early evolution of Sauropodomorpha. PLoS ONE 4:e4397+.

Matsumoto, R., Suzuki, S., Tsogtbaatar, K. and Evans, S. 2009. New material of the enigmatic reptile Khurendukhosaurus (Diapsida: Choristodera) from Mongolia. Naturwissenschaften 96:233-242.

Mayor, A. 2000. The First Fossil Hunters Paleontology in Greek and Roman Times. Princeton University Press.

Mayr, E. 1953. Methods and Principles of Systematic Zoology. McGraw Hill, New York.

Mayr, G. 2005. A new cypselomorph bird from the Middle Eocene of Germany and the early diversification of avian aerial insectivores. The Condor 107:342-352.

Mayr, G., Rana, R. S., Rose, K. D., Sahni, A., Kumar, K., Singh, L., and Smith, T. 2010. Quercypsitta-Like birds from the Early Eocene of India (Aves, ?psittaciformes). Journal of Vertebrate Paleontology 30:467-478.

Mayr, G. 2010b. Phylogenetic relationships of the paraphyletic 'caprimulgiform' birds (nightjars and allies). Journal of Zoological Systematics and Evolutionary Research 48:126-137.

Mayr, G. 2011a. The phylogeny of charadriiform birds (shorebirds and allies) - reassessing the conflict between morphology and molecules. Zoological Journal of the Linnean Society 161:916-934.

Mayr, G. 2011b. Cenozoic mystery birds - on the phylogenetic affinities of bony-toothed birds (Pelagornithidae). Zoologica Scripta 40:448-467.

Mayr, G. and Mourer-Chauviré, C. 2004. Unusual tarsometatarsus of a mousebird from the Paleogene of France and the relationships of Selmes Peters, 1999. Journal of Vertebrate Paleontology 24: 366-372.

Maurício, G. N., Areta, J. I., Bornschein, M. R., and Reis, R. E. 2012. Morphology-based phylogenetic analysis and classification of the family Rhinocryptidae (Aves: Passeriformes). Zoological Journal of the Linnean Society 166:377-432.

McDonald, A. T., Kirkland, J. I., DeBlieux, D. D., Madsen, S. K., Cavin, J., Milner, A. R. C., and Panzarin, L. 2010. New basal iguanodonts from the cedar mountain formation of utah and the evolution of Thumb-Spiked dinosaurs. PLoS ONE 5:e14075+.

Meier, R., Kores, P., and Darwin, S. 1991. Homoplasy slope ratio: A better measurement of observed homoplasy in cladistic analyses. Systematic Zoology 40. 
Mendeley 2013. Compare Mendeley. http://www.mendeley.com/compare-mendeley/

Michener, C.D. 1953. Life-history studies in insect systematics. Systematic Zoology 2:112118.

Mickevich, M.F. 1978. Taxonomic congruence. Systematic Zoology 27:143-158.

Mickevich, M.F. and Farris J.S. 1981. The implications of congruence in Menidia. Systematic Zoology 30:351-370.

Microsoft 2013. About Microsoft Academic Search http://academic.research.microsoft.com/About/help.htm\#5

Mihaljevic M., Jerjen I. and A.B. Smith 2011. The test architecture of Clypeaster (Echinoidea, Clypeasteroida) and its phylogenetic significance. Zootaxa 2983: 21-38

Mitteroecker, P. and Bookstein, F. 2007. The conceptual and statistical relationship between modularity and morphological integration. Systematic Biology 56:818-836.

Mitteroecker, P. and Bookstein, F. 2008. The evolutionary role of modularity and integration in the hominoid cranium. Evolution 62:943-958.

Miyamoto, M. M. and Fitch, W. M. 1995. Testing species phylogenies and phylogenetic methods with congruence. Systematic Biology 44:64-76.

Mooi, R.D. and Gill, A.C. 2010. Phylogenies without synapomorphies - a crisis in fish systematics: Time to show some character. Zootaxa 2450:26-40.

Moore, M. J., Hassan, N., Gitzendanner, M. A., Bruenn, R. A., Croley, M., Vandeventer, A., Horn, J. W., Dhingra, A., Brockington, S. F., Latvis, M., Ramdial, J., Alexandre, R., Piedrahita, A., Xi, Z., Davis, C. C., Soltis, P. S., and Soltis, D. E. 2011. Phylogenetic analysis of the plastid inverted repeat for 244 species: Insights into Deeper-Level angiosperm relationships from a long, slowly evolving sequence region. International Journal of Plant Sciences 172:541-558.

Mounce, R. 2010. The continued growth of phylogenetic information. Young Systematists' Forum, 2010, London http://prezi.com/1sOlkatmc30t/the-continued-growth-ofphylogenetic-information/ [Conference Slides]

Mounce, R. 2011a. Phylogenetic congruence between cranial and postcranial characters in archosaur systematics. Society of Vertebrate Paleontology Annual Meeting, p163. [Conference Abstract]

Mounce, R. 2011b. Nullius in Calculo. Talk presented at the Eleventh Systematics Association Biennial Meeting, Dublin. http://prezi.com/rwyvfb9bvec_/nullius-in-calculo/

Mounce, R. [data] 2013a. BMC phylogeny paper corpus. https://github.com/rossmounce/BMC_phylogeny_corpus

Mounce, R. [data] 2013b. Article PDF file sizes. https://github.com/rossmounce/thesis_ESM/blob/master/lit_search_chapter/forR_Article pdfFileSizes.csv

Mounce, R. [data] 2013c. Web of Knowledge Winclada search data https://github.com/rossmounce/thesis_ESM/blob/master/lit_search_chapter/wok_wincla da.bib

Mounce, R. [data] 2013d. Scopus Winclada search data https://github.com/rossmounce/thesis_ESM/blob/master/lit_search_chapter/scopus_win clada.bib 
Mounce, R. [data], 2013e. Phylogeny publishers data exported from Web of Knowledge https://github.com/rossmounce/thesis_ESM/blob/master/lit_search_chapter/phylogen_p ublishers_in_wok.csv

Mounce, R. [data], 2013f. Longtail data on the distibution of phylogeny articles https://github.com/rossmounce/thesis_ESM/blob/master/lit_search_chapter/long_tail_p hylogen_articles_data.csv

Mounce, R. [data] 2013g. Bibliographic data for vertebrate morphology-based phylogenetic studies. http://www.citeulike.org/user/rossmounce/tag/morphophylogeny

Mounce, R. [data] 2013h. Bibliographic data for invertebrate morphology-based phylogenetic studies. http://www.citeulike.org/user/rossmounce/tag/nvmorphophylogeny

Mounce, R. 2013. Content Mining. Talk given in Brussels at the Licences for Europe Stakeholder dialogue for the European Commission. http://www.slideshare.net/rossmounce/content-mining [Conference Talk]

Muller, J. and Reisz, R.R. 2006. The phylogeny of early eureptiles: Comparing parsimony and Bayesian approaches in the investigation of a basal fossil clade. Systematic Biology 55:503-511.

Naylor, G.J.P. and Adams, D.C. 2001. Are the fossil data really at odds with the molecular data? Morphological evidence for Cetartiodactyla phylogeny reexamined. Systematic Biology 50: 444-453.

Near, T. J., Pesavento, J.J. and Cheng, C.-H.C. 2003. Mitochondrial DNA, morphology, and the phylogenetic relationships of antarctic icefishes (Notothenioidei: Channichthyidae). Molecular Phylogenetics and Evolution 28: 87-98.

Near, T.J. 2009. Conflict and resolution between phylogenies inferred from molecular and phenotypic data sets for hagfish, lampreys, and gnathostomes. Journal of Experimental Zoology 312:749-761.

Nee, S., and May, R. M. 1997. Extinction and the loss of evolutionary history. Science 278:692:694.

Nelson, G.J., "Cladism" as a philosophy of classification. Systematic Zoology 20:373-376, 1971.

Nelson, G.J. 1969. Origin and diversification of teleostean fishes Annals of the New York Academy of Sciences 167: 18-30.

Nelson, G.J. 1970. Outline of a theory of comparative biology, Systematic Zoology 19:373384.

Newman, L. 2012. Introducing our Panton Fellows. http://blog.okfn.org/2012/03/30/introducing-our-panton-fellows/

Ngamskulrungroj, P., Gilgado, F., Faganello, J., Litvintseva, A. P., Leal, A. L., Tsui, K. M., Mitchell, T. G., Vainstein, M. H., and Meyer, W. 2009. Genetic diversity of the cryptococcus species complex suggests that Cryptococcus gattii deserves to have varieties. PLOS ONE 4:e5862+.

Nichols, R. 2001. Gene trees and species trees are not the same. Trends in Ecology and Evolution 16:358-364.

Nixon K. C. 1999. The Parsimony Ratchet, a new method for rapid parsimony analysis. Cladistics 15:407-414. 
Nixon, K. C. 2002. WinClada, version 1.00. 08. Published by the author, Ithaca, New York.

Novacek, M. J. 1992. Fossils, topologies, missing data, and the higher level phylogeny of eutherian mammals. Systematic Biology 41:58-73.

Novacek, M. J., and Wheeler, Q. D. 1992. Extinct taxa-accounting for 99.9990 . . \% of the earth's biota. pp. 1-16 in Extinction and Phylogeny. M. J. Novacek and Q. D. Wheeler (eds.) Columbia University Press, New York.

Nygren, A., Sundkvist, T., Mikac, B. and Pleijel, F. 2010. Two new and two poorly known autolytines (Polychaeta:Syllidae) from Madeira and the Mediterranean Sea. Zootaxa 2640:35-52.

O'Connor, P.M., Sertich, J.J.W., Stevens, N.J., Roberts, E.M., Gottfried, M.D., Hieronymus, T.L., Jinnah, Z.A., Ridgely, R., Ngasala, S.E. and Temba, J. 2010. The evolution of mammal-like crocodyliforms in the Cretaceous period of Gondwana. Nature 466: 748-751.

O'Leary, M.A., Gatesy, J., and Novacek, M.J. 2003. Are the dental data really at odds with the molecular data? Morphological evidence for whale phylogeny $(\mathrm{Re}) \mathrm{Reexamined.}$ Systematic Biology 52:853-864.

O'Leary, M. A. and Kaufman, S. 2011. MorphoBank: phylophenomics in the "cloud". Cladistics 27:529-537.

O'Leary, M. A., Bloch, J. I., Flynn, J. J., Gaudin, T. J., Giallombardo, A., Giannini, N. P., Goldberg, S. L., Kraatz, B. P., Luo, Z.-X. X., Meng, J., Ni, X., Novacek, M. J., Perini, F. A., Randall, Z. S., Rougier, G. W., Sargis, E. J., Silcox, M. T., Simmons, N. B., Spaulding, M., Velazco, P. M., Weksler, M., Wible, J. R., and Cirranello, A. L. 2013. The placental mammal ancestor and the post-K-pg radiation of placentals. Science 339:662667.

O'Meara, B. 2009. TreeBASE mirror http://www.brianomeara.info/treebase [accessed December 12th 2009]

Olson, E. 1991. George Gaylord Simpson 1902-1984, A Biographical Memoir. National Academy of Sciences.

Orlando, L., Ginolhac, A., Zhang, G., Froese, D., Albrechtsen, A., Stiller, M., Schubert, M., Cappellini, E., Petersen, B., Moltke, I., Johnson, P. L. F., Fumagalli, M., Vilstrup, J. T., Raghavan, M., Korneliussen, T., Malaspinas, A.-S., Vogt, J., Szklarczyk, D., Kelstrup, C. D., Vinther, J., Dolocan, A., Stenderup, J., Velazquez, A. M. V., Cahill, J., Rasmussen, M., Wang, X., Min, J., Zazula, G. D., Seguin-Orlando, A., Mortensen, C., Magnussen, K., Thompson, J. F., Weinstock, J., Gregersen, K., Roed, K. H., Eisenmann, V., Rubin, C. J., Miller, D. C., Antczak, D. F., Bertelsen, M. F., Brunak, S., Al-Rasheid, K. A. S., Ryder, O., Andersson, L., Mundy, J., Krogh, A., Gilbert, Kjaer, K., Sicheritz-Ponten, T., Jensen, L. J., Olsen, J. V., Hofreiter, M., Nielsen, R., Shapiro, B., Wang, J., and Willerslev, E. 2013. Recalibrating equus evolution using the genome sequence of an early middle pleistocene horse. Nature 499:74-78.

Osi, A. and Makadi, L. 2009. New remains of Hungarosaurus tormai (Ankylosauria, Dinosauria) from the Upper Cretaceous of Hungary: Skeletal reconstruction and body mass estimation. Paleontologische Zeitschrift 83:227-245.

Page, R.D.M. 1996. On consensus, confidence, and 'total evidence'. Cladistics 12:83-92.

Pamilo, P., Nei, M. 1988. Relationships between gene trees and species trees. Molecular Biology and Evolution 5:568-583. 
Paradis, E., Claude, J., and Strimmer, K. 2004. APE: Analyses of phylogenetics and evolution in r language. Bioinformatics 20:289-290.

Parenti, L.R. 2008. A phylogenetic analysis and taxonomic revision of ricefishes, oryzias and relatives (Beloniformes, Adrianichthyidae). Zoological Journal of the Linnean Society 154:494-610.

Pattengale, N.D., Gottlieb, E.J. and Moret, B.M.E. 2007. Efficiently computing the Robinson-Foulds metric. Journal of Computational Biology 14:724-735.

Patterson, C. 1981. Significance of fossils in determining evolutionary relationships. Annual Review of Ecology and Systematics 12:195-223.

Peach, M.B. and Rouse G.W. 2004. Phylogenetic trends in the abundance and distribution of pit organs of elasmobranchs. Acta Zoologica 85:233-244

Peñalver, E., J. Ortega-Blanco, A. Nel, and X. Delclòs 2010. Mesozoic evaniidae (Insecta: Hymenoptera) in spanish amber: Reanalysis of the phylogeny of the evanioidea. Acta Geologica Sinica 84:809-827.

Pepato, A., da Rocha, C., and Dunlop, J. 2010. Phylogenetic position of the acariform mites: sensitivity to homology assessment under total evidence. BMC Evolutionary Biology 10:235+.

Perrichot, V., Nel, A., and Quicke, D. 2009. New braconid wasps from french Cretaceous amber (Hymenoptera, Braconidae): synonymization with eoichneumonidae and implications for the phylogeny of ichneumonoidea. Zoologica Scripta 38:79-88.

Phillips, M.J., Bennett, T.H. and Lee, M.S.Y. 2009. Molecules, morphology, and ecology indicate a recent, amphibious ancestry for echidnas. Proceedings of the National Academy of Sciences of the United States of America 106:17089-17094.

Phillips, A. J., Arauco-Brown, R., Oceguera-Figueroa, A., Gomez, G. P., Beltrán, M., Lai, Y.-T., and Siddall, M. E. 2010. Tyrannobdella rex n. gen. n. sp. and the evolutionary origins of mucosal leech infestations. PLOS ONE 5:e10057+.

Piel, W. H., Donoghue, M., and Sanderson, M. 2002. TreeBASE: A database of phylogenetic information. Research Report from the National Institute for Environmental Studies 171:41-47.

Pine, R. H., Timm, R. M., and Weksler, M. 2012. A newly recognized clade of transAndean oryzomyini (Rodentia: Cricetidae), with description of a new genus. Journal of Mammalogy 93:851-870.

Pinna, M.C.C. 1991. Concepts and tests of homology in the cladistic paradigm. Cladistics 7:367-394.

Pirie, M. D., Humphreys, A. M., Galley, C., Barker, N. P., Verboom, G. A., Orlovich, D., Draffin, S. J., Lloyd, K., Baeza, C. M., Negritto, M., Ruiz, E., Sanchez, J. H. C., Reimer, E., and Linder, H. P. 2008. A novel supermatrix approach improves resolution of phylogenetic relationships in a comprehensive sample of danthonioid grasses. Molecular Phylogenetics and Evolution 48:1106-1119.

Pisani, D. 2002. Comparing and combining trees and data in phylogenetic analysis. PhD thesis. University of Bristol.

Pisani, D., Benton, M., and Wilkinson, M. 2007. Congruence of morphological and molecular phylogenies. Acta Biotheoretica 55:269-281-281. 
Piwowar, H. A., Day, R. S., and Fridsma, D. B. 2007. Sharing detailed research data is associated with increased citation rate. PLOS ONE 2:e308+.

Piwowar, H. A., Vision, T. J., and Whitlock, M. C. 2011. Data archiving is a good investment. Nature 473:285.

Piwowar, H. A., Vision, T.J. 2013. Data reuse and the open data citation advantage. PeerJ $1: \mathrm{e} 175$

Piwowar, H. 2013. Altmetrics: Value all research products. Nature 493:159

Planet, P.J. 2006 Tree disagreement: Measuring and testing incongruence in phylogenies. Journal of Biomedical Informatics 39:86-102.

Planet, P. J. and Sarkar, I.N. 2005. mILD: a tool for constructing and analyzing matrices of pairwise phylogenetic character incongruence tests. Bioinformatics 21:4423-4424.

Poe, S. 1996. Data set incongruence and the phylogeny of crocodilians. Systematic Biology 45:393-414.

Poe, S., and Wiens, J. J. 2000. Character selection and the methodology of morphological phylogenetics pp 20-36. In: J. J. Wiens (ed.) Phylogenetic analysis of morphological data. Smithsonian Institution Press, Washington, DC.

Pogue, M.G. and Mickevich, M.F. 1990. Character definitions and character state delineation: The bête noire of phylogenetic inference. Cladistics 6:319-361.

Pol, D. and Escapa, I. H. 2009. Unstable taxa in cladistic analysis: identification and the assessment of relevant characters. Cladistics 25:515-527.

Ponder, W.F., Clark, S.A, Eberhard, S., Studdert, J.B. A radiation of hydrobiid snails in the caves and streams at Precipitous Bluff, southwest Tasmania, Australia (Mollusca: Caenogastropoda: Rissooidea: Hydrobiidae s.I.). Zootaxa 1074:1-66.

Poyato-Ariza, F. J. 2003. Dental characters and phylogeny of pycnodontiform fishes. Journal of Vertebrate Paleontology 23:937-940.

Poyato-Ariza, F. J., Grande, T., and Diogo, R. 2010. Gonorynchiform interrelationships: Historic overview, analysis, and revised systematics of the group. In: Gonorynchiformes and Ostariophysan Relationships A Comprehensive Review pp. 227-337.

Pradel, A., Tafforeau, P., Maisey, J. G., and Janvier, P. 2011. A new Paleozoic Symmoriiformes (Chondrichthyes) from the Late Carboniferous of Kansas (USA) and cladistic analysis of early chondrichthyans. PLOS ONE 6:e24938+.

Prager, E.M. and Wilson, A.C. 1988. Ancient origin of lactalbumin from lysozyme: Analysis of DNA and amino acid sequences. Journal of Molecular Evolution 27:326-335.

Prentice, T. R. and Redak, R. A. 2012. Esophyllas, a new genus of erigonine spiders from southern California (Araneae: Linyphiidae: Erigoninae). Zootaxa 3265:1-21.

Pretti, V. Q., Calcagnotto, D., Toledo-Piza, M., and de Almeida-Toledo, L. F. 2009. Phylogeny of the neotropical genus Acestrorhynchus (Ostariophysi: Characiformes) based on nuclear and mitochondrial gene sequences and morphology: a total evidence approach. Molecular Phylogenetics and Evolution 52:312-320.

Prevosti, F. J. 2010. Phylogeny of the large extinct south american canids (Mammalia, Carnivora, Canidae) using a "total evidence" approach. Cladistics 26:456-481.

Prideaux, G. J. and Warburton, N. M. 2010. An osteology-based appraisal of the 
phylogeny and evolution of kangaroos and wallabies (Macropodidae: Marsupialia). Zoo. J. Linn. Soc. 159:954-987.

Puértolas, E., Canudo, J.I. and Cruzado-Caballero, P. 2011. A new crocodylian from the late Maastrichtian of Spain: Implications for the initial radiation of crocodyloids. PLoS ONE 6: e20011+.

Pujos, F., de luliis, G., Argot, C. and Werdelin, L. 2007. A peculiar climbing Megalonychidae from the Pleistocene of Peru and its implication for sloth history. Zoological Journal of the Linnean Society 149:179-235.

Quicke, D.L., Jones, O.R. and Epstein, D.R. 2007. Correcting the problem of false incongruence due to noise imbalance in the incongruence length difference (ILD) test. Systematic Biology 56:496-503.

Ragan, M. 1992. Matrix representation in reconstructing phylogenetic relationships among the eukaryotes. Biosystems 28:47-55.

Ragan, M.A., Mclnerney, J.O. and Lake, J.A. 2009. The network of life: Genome beginnings and evolution. Philosophical Transactions of the Royal Society B: Biological Sciences 364:2169-2175.

Ragsdale, E. J. and Baldwin, J. G. 2010. Resolving phylogenetic incongruence to articulate homology and phenotypic evolution: a case study from nematoda. Proceedings of the Royal Society B: Biological Sciences 277:1299-1307.

Ramirez, M.J. 2006. Further problems with the incongruence length difference test: "hypercongruence" effect and multiple comparisons. Cladistics 22:289-295.

Robinson, D.R. and Foulds, L.R. 1981. Comparison of phylogenetic trees. Mathematical Biosciences 53:131-147.

Rodrigo A. G., Kelly-Borges, M., Bergquist, P.R. and Bergquist, P.L. 1993. A randomization test of the null hypothesis that two cladograms are sample estimates of a parametric phylogenetic tree. New Zealand Journal of Botany 31:257-268.

Ronquist, F., Klopfstein, S., Vilhelmsen, L., Schulmeister, S., Murray, D. L., and Rasnitsyn, A. P. 2012. A Total-Evidence approach to dating with fossils, applied to the early radiation of the hymenoptera. Systematic Biology 61:973-999.

Rossello, F. and Valiente, G. 2006. The transposition distance for phylogenetic trees. arXiv:q-bio/0604024v1 [q-bio.PE]

Ruta, M. and Coates, M.I. 2007. Dates, nodes and character conflict: Addressing the lissamphibian origin problem. Journal of Systematic Palaeontology 5:69-122.

Sánchez-Villagra, M.R. and Williams, B.A. 1998. Levels of homoplasy in the evolution of the mammalian skeleton. Journal of Mammalian Evolution 5:113-126.

Sánchez-Villagra, M.R., Horovitz, I. and Motokawa, M. 2006. A comprehensive morphological analysis of talpid moles (Mammalia) phylogenetic relationships. Cladistics 22:59-88.

Sanderson, M. J. and Donoghue, M. J. 1989. Patterns of variation in levels of homoplasy. Evolution 43:1781-1795.

Sang, T. 1995. New measurements of distribution of homoplasy and reliability of parsimonious cladograms. Taxon 44: 77-82.

Sansom, R.S., Gabbott, S.E. and Purnell, M.A. 2010. Non-random decay of chordate 
characters causes bias in fossil interpretation. Nature 463:797-800.

Schaeffer, B., Hecht, B.M., and Eldredge, N. 1972. Phylogeny and paleontology, pp. 3146. In: T. Dobzhansky, B.M. Hecht, and W. Steere (eds.), Evolutionary Biology vol 6, Springer US.

Schliep, K. P. 2011. Phangorn: phylogenetic analysis in R. Bioinformatics 27:592-593.

Schuh, R. T., Weirauch, C., and Wheeler, W. C. 2009. Phylogenetic relationships within the cimicomorpha (hemiptera: Heteroptera): a total-evidence analysis. Systematic Entomology 34:15-48.

SciVerse, 2013. SciVerse Titles List http://files.sciverse.com/documents/xlsx/title_list.xlsx

Scotland, R. W., Olmstead, R. G., and Bennett, J. R. 2003. Phylogeny reconstruction: The role of morphology. Systematic Biology 52:539-548.

Seiffert, E. 2007. A new estimate of afrotherian phylogeny based on simultaneous analysis of genomic, morphological, and fossil evidence. BMC Evolutionary Biology 7:224+.

Sereno, P.C. and Brusatte, S.L. 2008. Basal abelisaurid and carcharodontosaurid theropods from the Lower Cretaceous Elrhaz formation of Niger. Acta Palaeontologica Polonica 53:15-46.

Sharkey, M.J. 2005. A new species of Agathirsia Westwood (Hymenoptera: Braconidae: Agathidinae) from Mexico. Zootaxa 1070:43-47.

Shimada, K. 2005. Phylogeny of lamniform sharks (Chondrichthyes: Elasmobranchii) and the contribution of dental characters to lamniform systematics. Paleontological Research 9:55-72.

Shimodaira, H. and Hasegawa, M. 1999. Multiple comparisons of Log-Likelihoods with applications to phylogenetic inference. Molecular Biology and Evolution 16:1114+.

Shimodaira, H. 2002. An approximately unbiased test of phylogenetic tree selection. Systematic Biology 51:492-508.

Siddall, M. 1998. The follies of ancestor worship. Nature Debates. http://www.nature.com/nature/debates/fossil/fossil_3.html

Siddall, M. E. 1997. Prior agreement: Arbitration or arbitrary? Systematic Biology 46:765+.

Siegel, S. and Castellan, N.J. 1998. Nonparametric Statistics for the Behavioural Sciences. McGraw-Hill, New York.

Sigwart, J. D. and M. D. Sutton 2007. Deep molluscan phylogeny: synthesis of palaeontological and neontological data. Proceedings of the Royal Society B: Biological Sciences 274:2413-2419.

Simmons, N.B. and Conway, T.M. 2001. Phylogenetic relationships of mormoopid bats (Chiroptera: Mormoopidae) based on morphological data. Bulletin of the American Museum of Natural History 258:1-100.

Simmons, N.B., Seymour, K.L., Habersetzer, J. and Gunnell, G.F. 2008. Primitive early Eocene bat from Wyoming and the evolution of flight and echolocation. Nature 451:818-821.

Simpson, G.G. (1937) Patterns of Phylectic Evolution. Bull. Geol. Soc. Amer. 48:308-309.

Simpson, G.G. (1944) Tempo and Mode in Evolution. New York: Columbia Univ. Press.

Skutschas, P. P., and Y. M. Gubin. 2012. A New Salamander from the Late Paleocene - 
Early Eocene of Ukraine. Acta Palaeontologica Polonica. 57:135-148

Smith, A. B. 1994. Systematics and the fossil record: Documenting evolutionary patterns. Blackwell Scientific, Oxford, U.K.

Smith, V. 2009. Data publication: Towards a database of everything. BMC Research Notes $2: 113+$.

Smith, N. D. 2010. Phylogenetic analysis of pelecaniformes (aves) based on osteological data: Implications for waterbird phylogeny and fossil calibration studies. PLoS ONE 5:e13354+.

Smith, N. D. and Turner, A. H. 2005 Morphology's Role in Phylogeny Reconstruction: Perspectives from Paleontology. Systematic Biology 54:166-173.

Smith, N. D. and Pol, D. 2007. Anatomy of a basal sauropodomorph dinosaur from the early Jurassic Hanson formation of Antarctica. Acta Palaeontologica Polonica 52:657674.

Sokal, R.R. and Sneath, P.H.A. 1963. Principles of Numerical Taxonomy. W.H. Freeman \& Co., San Francisco.

Song, H. and Bucheli, S.R. 2010. Comparison of phylogenetic signal between male genitalia and non-genital characters in insect systematics. Cladistics 26:23-35.

Spangler, R. E. and Olmstead, R. G. 1999. Phylogenetic analysis of Bignoniaceae based on the cpDNA gene sequences rbcL and ndhF. Annals of the Missouri Botanical Garden 86: 33-46.

Sparks, J.S. 2008. Phylogeny of the cichlid subfamily Etroplinae and taxonomic revision of the Malagasy cichlid genus Paretroplus (Teleostei: Cichlidae). Bulletin of the American Museum of Natural History 314:1+.

Spaulding, M., O'Leary, M.A. and Gatesy, J. 2009. Relationships of Cetacea (Artiodactyla) among mammals: Increased taxon sampling alters interpretations of key fossils and character evolution. PLOS ONE 4:e7062+.

Spaulding, M and Flynn, J. 2012. Phylogeny of the Carnivoramorpha: The impact of postcranial characters. Journal of Systematic Palaeontology 10:653-677.

Springer, M.S., Burk-Herrick, A., Meredith, R., Eizirik, E., Teeling, E., O'Brien, S.J. and Murphy, W.J. 2007. The adequacy of morphology for reconstructing the early history of placental mammals. Systematic Biology 56:673-684.

Springer, M. S., Meredith, R. W., Gatesy, J., Emerling, C. A., Park, J., Rabosky, D. L., Stadler, T., Steiner, C., Ryder, O. A., Janečka, J. E., Fisher, C. A., and Murphy, W. J. 2012. Macroevolutionary dynamics and historical biogeography of primate diversification inferred from a species supermatrix. PLOS ONE 7:e49521+.

Stackoverflow, 2012 How do I grep in parallel? http://stackoverflow.com/questions/11898949/how-do-i-grep-in-parallel? answertab=active\#tab-top

Steel, M.A. and Penny, D. 1993. Distributions of tree comparison metrics - some new results. Systematic Biology 42:126-141.

Stoltzfus, A., O'Meara, B., Whitacre, J., Mounce, R., Gillespie, E., Kumar, S., Rosauer, D., and Vos, R. 2012. Sharing and re-use of phylogenetic trees (and associated data) to facilitate synthesis. BMC Research Notes 5:574+. 
Sues, H.-D. and Averianov, A. 2009. A new basal hadrosauroid dinosaur from the Late Cretaceous of Uzbekistan and the early radiation of duck-billed dinosaurs. Proceedings of the Royal Society of London. Series B: Biological Sciences 276:2549-2555.

Sues, H.-D. and Reisz, R.R. 2008. Anatomy and phylogenetic relationships of Sclerosaurus armatus (Amniota: Parareptilia) from the Buntsandstein (Triassic) of Europe. Journal of Vertebrate Paleontology 28:1031-1042.

Sullivan J., 1996 Combining data with different distributions of among-site rate variation Systematic Biology 45:375-380

Swofford, D.L. 2002. PAUP*: Phylogenetic analysis using parsimony ( ${ }^{*}$ and other methods). Version 4. Sinauer Associates, Sunderland, Massachusetts.

Swofford, D.L., Olsen, G. J., Waddell, P. G. and Hillis, D. M. 1996. Phylogenetic inference. In: D.M. Hillis, C. Moritz and B.K. Mable, editors. Molecular Systematics. Sinauer Associates. Sunderland, Massachusetts: p. 407-514.

Tange, O. 2011. GNU Parallel - The Command-Line Power Tool. Login: The USENIX Magazine Feb:42-47.

Templeton, A.R. 1983. Phylogenetic inference from restriction endonuclease cleavage site maps with particular reference to the evolution of humans and the apes. Evolution $37: 221-244$.

Thomson, R. C. and Shaffer, H. B. 2010. Sparse supermatrices for phylogenetic inference: Taxonomy, alignment, rogue taxa, and the phylogeny of living turtles. Systematic Biology 59:42-58.

Thomson Reuters, 2013. Web of Knowledge: Who We Are http://wokinfo.com/about/whoweare/

Thorley, J.L. and Page, R.D.M. 2000. RadCon: Phylogenetic tree comparison and consensus. Bioinformatics 16:486-487.

Triapitsyn, S. V., Vickerman, D.B., Heraty, J. M., Logarzo, G.A. 2006. A new species of Gonatocerus (Hymenoptera: Mymaridae) parasitic on proconiine sharpshooters (Hemiptera: Cicadellidae) in the New World. Zootaxa 1158:55-67.

Trueb, L. and A. M. Baez 2006. Revision of the Early Cretaceous Cordicephalus from Israel and an assessment of its relationships among pipoid frogs. Journal of Vertebrate Paleontology 26:44-59.

Turner, A.H. and Sertich, J.J.W. 2010. Phylogenetic history of Simosuchus clarki (Crocodyliformes: Notosuchia) from the late cretaceous of madagascar. Journal of Vertebrate Paleontology 30: 177-236.

Vallin, G. and Laurin, M. 2004. Cranial morphology and affinities of Microbrachis, and a reappraisal of the phylogeny and lifestyle of the first amphibians. Journal of Vertebrate Paleontology 24:56-72.

van der Linde, K., Houle, D., Spicer, G. S., and Steppan, S. J. 2010. A supermatrix-based molecular phylogeny of the family drosophilidae. Genetics Research 92:25-38.

Vea, I. M. and D. A. Grimaldi 2012. Phylogeny of ensign scale insects (Hemiptera: Coccoidea: Ortheziidae) based on the morphology of recent and fossil females. Systematic Entomology 37:758-783.

Venczel, M. 2008. A new salamandrid amphibian from the Middle Miocene of Hungary and 
its phylogenetic relationships. Journal of Systematic Palaeontology 6:41-59.

Vermeij, G.J. and Carlson, S.J. 2000. The muricid gastropod subfamily Rapaninae: Phylogeny and ecological history. Paleobiology 26:19-46.

Vidal, N., and Lecointre, G. 1998. Weighting and congruence: A case study based on three mitochondrial genes in pitvipers. Molecular Phylogenetics and Evolution 9: 366 -374.

Vieira, G.H.C., Colli, G.R. and Bao, S.N. 2005. Phylogenetic relationships of corytophanid lizards (Iguania, Squamata, Reptilia) based on partitioned and total evidence analyses of sperm morphology, gross morphology, and DNA data. Zoologica Scripta 34:605-625.

Vilhelmsen, L., Perrichot, V., and Shaw, S. R. 2010. Past and present diversity and distribution in the parasitic wasp family megalyridae (Hymenoptera). Systematic Entomology 35:658-677.

von Reumont, B.M., Jenner, R.A., Wills, M.A., Dell'Ampio, E., Pass, G., Ebersberger, I., Meyer, B., Koenemann, S., Iliffe, T.M., Stamatakis, A., Niehuis, O., Meusemann, K. and Misoff, B. 2012. Pancrustacean phylogeny in the light of new phylogenomic data: support for Remipedia as the possible sister group of Hexapoda. Molecular Biology and Evolution 29: 1031-1045.

W3C, 1991. http://www.w3.org/Protocols/HTTP/AsImplemented.html

Walters, W. H. 2011. Comparative Recall and Precision of Simple and Expert Searches in Google Scholar and Eight Other Databases. Portal: libraries and the academy 11:9711006.

Ward, C.V. 1997. Functional anatomy and phyletic implications of the hominoid trunk and hindlimb pp. 101-130. In: B. Begun, C. Ward, and M. Rose (eds.) Function, Phylogeny, and Fossils: Miocene Hominoid Evolution and Adaptations. Plenum Press, New York.

Waterman, M.S. and Smith, T.F. 1978. On the similarity of dendrograms. Journal of Theoretical Biology 73:789-800.

Webb, D.W. 2005. New Genera of Neotropical Therevidae (Insecta: Diptera). Zootaxa 1091:1-26.

Weksler, M. 2006. Phylogenetic relationships of oryzomine rodents (Muroidea: Sigmodontinae): Separate and combined analyses of morphological and molecular data. Bulletin of the American Museum of Natural History 296:1+.

Wheeler, Q.D. 1981. The ins and outs of character analysis: A response to Crisci and Stuessy. Systematic Botany 6: 297-306.

Wheeler, W C 1991. Congruence among data sets: A Bayesian Approach pp. $334-346$. In: M M Miyamoto and J Cracraft (eds.) Phylogenetic analysis of DNA sequences. Oxford University Press

Wheeler, W. C. 1999. Measuring topological congruence by extending character techniques. Cladistics 15:131-135.

White, E.P., Baldridge, E., Brym, Z.T. Locey, K.J., McGlinn, D.J. and Supp, S.R. 2013. Nine simple ways to make it easier to (re)use your data. Ideas in Ecology and Evolution. 6: $1-10$.

Whitlock, J. A. 2010. Phylogenetic relationships of the Eocene percomorph fishes †Priscacara and †Mioplosus. Journal of Vertebrate Paleontology 30:1037-1048.

Wible, J. R., Rougier, G.W., Novacek, M.J. and Asher, R.J. 2009. The eutherian mammal 
Maelestes gobiensis from the Late Cretaceous of Mongolia and the phylogeny of Cretaceous Eutheria. Bulletin of the American Museum of Natural History 327:1-123.

Wickham, H. 2011. The Split-Apply-combine strategy for data analysis. Journal of Statistical Software 40:1-29.

Wiens, J. J. 2003a. Incomplete taxa, incomplete characters, and phylogenetic accuracy: Is there a missing data problem? Journal of Vertebrate Paleontology 23:297-310.

Wiens, J. J. 2003b. Missing data, incomplete taxa, and phylogenetic accuracy. Systematic Biology 52:528-538.

Wiens, J. J. 2004. The role of morphological data in phylogeny reconstruction. Systematic Biology 53:653-661.

Wiens, J. J. \& Hillis, D.M. 1996. Accuracy of parsimony analysis using morphological data: a reappraisal. 21:237-253.

Wiens, J. J. and Hollingsworth, B. D. 2000. War of the iguanas: Conflicting molecular and morphological phylogenies and Long-Branch attraction in iguanid lizards. Systematic biology 49:143-159.

Wiens, J., Fetzner, J., Parkinson, C. and Reeder, T. 2005. Hylid frog phylogeny and sampling strategies for speciose clades. Systematic Biology 54:719-748.

Wilkinson, M. 1995a. Coping with abundant missing entries in phylogenetic inference using parsimony. Systematic Biology 44:501-514.

Wilkinson, M. 1995b. TAXEQ3: safe taxonomic reduction using taxonomic equivalence. Computer program published by the author. http://www.nhm.ac.uk/researchcuration/research/projects/software/

Wilkinson, M. 1997. Characters, congruence and quality: A study of Neuroanatomical and Traditional Data in Caecilian Phylogeny. Biological Reviews 72: 423-470.

Wilkinson, M. 2001 PICA 4.0: software and documentation. Department of Zoology, The Natural History Museum, London. http://www.nhm.ac.uk/researchcuration/research/projects/software/

Williams, W.T. and Clifford, H.T. 1971. On the comparison of two classifications of the same set of elements. Taxon 20: 519-522.

Wills, M.A. 1999. Congruence between phylogeny and stratigraphy: Randomization tests and the gap excess ratio. Systematic Biology 48:559-580.

Wilson, E.O. 1998. Consilience: The Unity of Knowledge. Alfred A. Knopf, New York.

Wilson, J. J. 2010. Assessing the value of DNA barcodes and other priority gene regions for molecular phylogenetics of lepidoptera. PLOS ONE 5:e10525+.

Winther, R. 2009. Character analysis in cladistics: Abstraction, reification, and the search for objectivity. Acta Biotheoretica 57:129-162-162.

Wolsan, M. and Sato, J. J. 2010. Effects of data incompleteness on the relative performance of parsimony and bayesian approaches in a supermatrix phylogenetic reconstruction of Mustelidae and Procyonidae (Carnivora). Cladistics 26:168-194.

Wood, H. M., Griswold, C. E., and Gillespie, R. G. 2012. Phylogenetic placement of pelican spiders (Archaeidae, Araneae), with insight into evolution of the " neck" and predatory behaviours of the superfamily Palpimanoidea. Cladistics 28:598-626. 
Worthy, T.H. 2009. Descriptions and phylogenetic relationships of two new genera and four new species of Oligo-Miocene waterfowl (Aves: Anatidae) from Australia. Zoological Journal of the Linnean Society 156:411-454.

Worthy, T and Scofield 2012. Twenty-first century advances in knowledge of the biology of moa (Aves:Dinornithiformes): a new morphological analysis and moa diagnoses revised. New Zealand Journal of Zoology, 39:87-153.

Yoder, A. D., Irwin, J. A., and Payseur, B. A. 2001. Failure of the ILD to determine data combinability for slow loris phylogeny. Systematic biology 50:408-424.

Young, M.T. and de Andrade. M.B. 2009. What is Geosaurus?: redescription of Geosaurus giganteus (Thalattosuchia: Metriorhynchidae) from the Upper Jurassic of Bayern, Germany. Zoological Journal of the Linnean Society 157:551-585.

Young, N.M. 2005. Estimating hominoid phylogeny from morphological data: character choice, phylogenetic signal and postcranial data pp 19-31. In: D.E. Lieberman, R.J. Smith, J. Kelley (eds.) Interpreting the Past: Essays on Human, Primate and Mammal Evolution in Honor of David Pilbeam. Brill Academic Publishers. Boston.

Zanno, L.E., Gillette, D.D., Albright, L.B. and Titus, A.L. 2009. A new North American therizinosaurid and the role of herbivory in 'predatory' dinosaur evolution. Proceedings of the Royal Society of London. Series B: Biological Sciences 276:3505-3511.

Zaretskii, K.A. 1965. Constructing a tree on the basis of a set of distances between the hanging vertices. Uspekhi Mat. Nauk 20:90-92.

Zhang, J. Y. 2006. Phylogeny of Osteoglossomorpha. Vertebrata PalAsiatica 44:43-59.

Zrzavy, J., Riha, P., Pialek, L., and Janouskovec, J. 2009. Phylogeny of Annelida (Lophotrochozoa): total-evidence analysis of morphology and six genes. BMC Evolutionary Biology 9:189+. 


\section{Appendices}

\section{Appendix 2.1}

(on next pages) Summary statistics for the 62 vertebrate morphological data sets analysed herein. Data set dimensions (numbers of taxa and informative characters) refer to the preprocessed matrices after applying safe taxonomic deletion rules (see text for details). ILD column reports the $p$-value resulting from an incongruence length difference test with 999 random partitions. TILD column reports $p$-value resulting from a topological incongruence length difference test (999 random partitions). IRD columns report the $p$-values resulting from incongruence relationship difference tests with 99 random partitions. IRD $D_{\mathrm{NND}+\mathrm{RF}}$ denotes the IRD test using the Robinson Foulds tree-to-tree distance for nearest neighbouring trees. IRD ${ }_{M R+R F}$ denotes the IRD test using the Robinson Foulds tree-to-tree distance for majority rule trees. IRD $D_{M R+A g D 1}$ denotes the IRD test using the maximum agreement subtree tree-to-tree distance for majority rule trees. $\mathrm{Cl}$ and $\mathrm{RI}$ columns give ensemble consistency and retention indices respectively for entire data sets. $\mathrm{pCl}$ and $\mathrm{pRI}$ columns give ensemble consistency and retention indices (respectively) for partitions of the data set (cranial or postcranial). Mean ci and Mean ri give the mean per character consistency and retention indices (respectively) for partitions of the data set (cranial or postcranial). MWU $p$-value gives the $p$-value from a Mann-Whitney $U$ test of cranial and postcranial per character ci values within each data set. pHER gives the homoplasy excess ratio for partitions of the data set (cranial or postcranial) derived from 999 randomized matrices. 


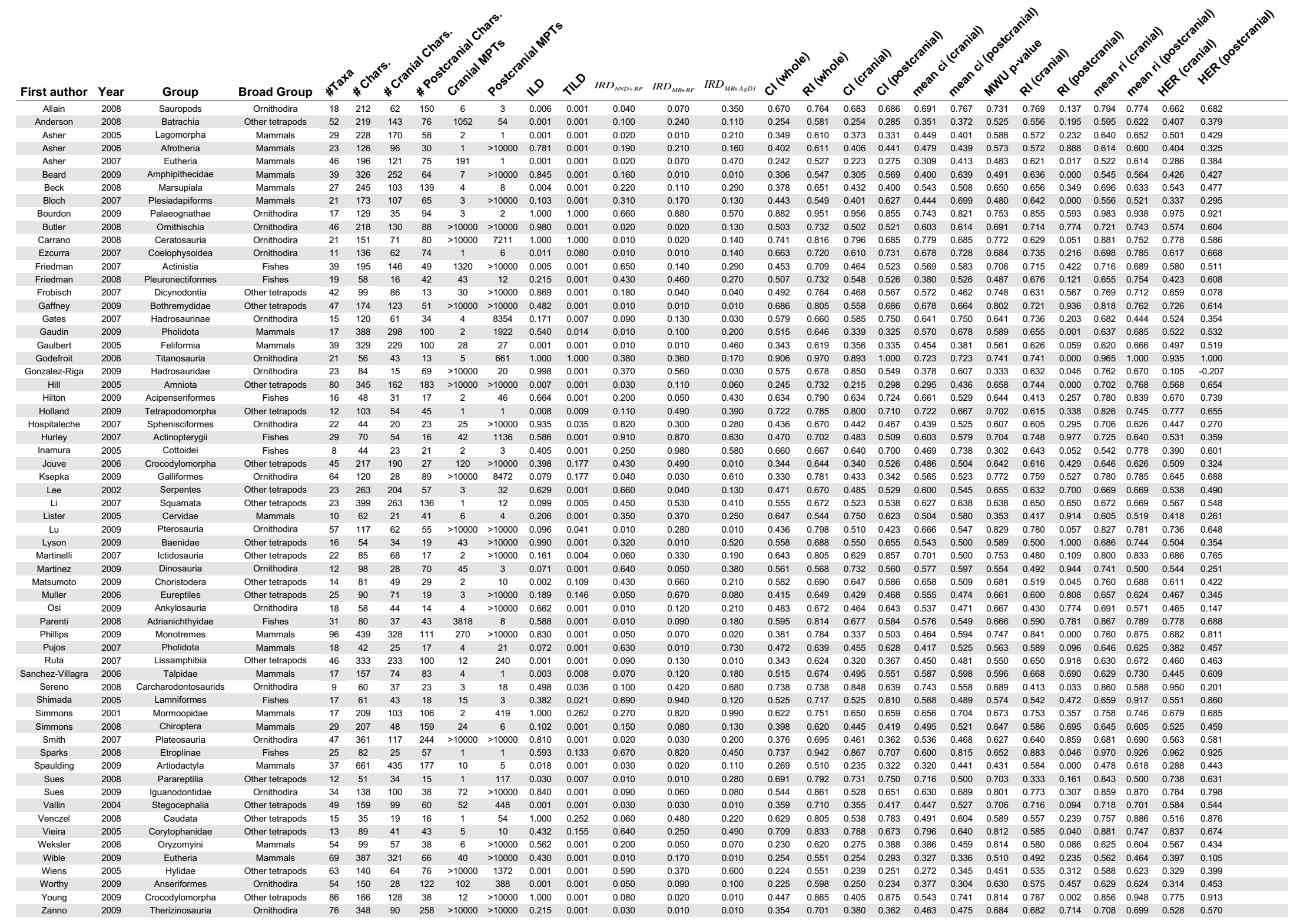




\section{Appendix 3.1 Data to reproduce PAUP bug}

Comparing the two trees below in PAUP* version 4.0b10 for Unix (and Windows) will reliably crash the program every single time, if they are compared using the AgD1 or AgD metrics. The message given is "Segmentation fault (core dumped)". Sometimes an 'apparent' AgD1 tree distance is printed in output log files as an impossibly large number e.g. 4294967293

Further instruction, including the exact commands to input into PAUP* to recreate this bug are given along with the data and tree files needed to reproduce it online at: https://github.com/rossmounce/extinct_extant_chapter/tree/master/PAUP_bug

tree Strict $=[\& U]$ (Acanthostega, (Proterogyrinus,Seymouria_baylorensis), ((Balanerpeton,Dendrerpeton,Tuditanus,(Asaphestera,(Microbrachis,Adelogyrinus)), (Hapsidoparion,Saxonerpeton),(Pantylus,Stegotretus), ((Cardiocephalus_peabodyi,Euryodus_primus),Euryodus_dalyae), (Pelodosotis,Micraroter),Rhynchonkos, Eocaecilia,Batropetes, Utaherpeton, ((Sauropleura_scalaris,Urocordylus),Ptyonius), (((Keraterpeton_galvani,Batrachiderpeton),Diceratosaurus, (Diplocaulus_magnicornis,Diploceraspis)),Scincosaurus),Brachydectes, Oestocephalus, $\mathrm{Phl}$ egethontia,Limnoscelis,Branchiosauridae,Micromelerpetontidae, (Ecolsonia,Acheloma,Tambachia),Eryops,Doleserpeton,Salamanders, (Frogs,Triadobatrachus),Albanerpetontidae,Micropholis,Eoscopus, Gerobatrachus,Platyrhin ops,Amphibamus), Greererpeton));

tree Strict $=[\& U]$ (Acanthostega, $($ Proterogyrinus, )(((((((((Tuditanus,Stegotretus),Pelodosotis),Saxonerpeton),Pantylus),Asaphestera),Batro petes),((Cardiocephalus_peabodyi,Euryodus_primus),

(Rhynchonkos,Eocaecilia))),Micraroter),Euryodus_dalyae),Utaherpeton), (Seymouria_baylorensis,Limnoscelis))),((Balanerpeton,Dendrerpeton), ((((((Hapsidoparion,Microbrachis),Brachydectes),Adelogyrinus),((Sauropleura_scalaris, (Ptyonius,Urocordylus,Scincosaurus),((Keraterpeton_galvani,Diceratosaurus), (Batrachiderpeton,(Diplocaulus_magnicornis,Diploceraspis)))), 
(Oestocephalus,Phlegethontia))),(Salamanders,Albanerpetontidae),

(Frogs,Triadobatrachus)),Doleserpeton,Gerobatrachus,Platyrhinops), Greererpeton, (Branchiosauridae,Micromelerpetontidae),Ecolsonia,Acheloma,Eryops,Micropholis,Eoscop us, Tambachia,Amphibamus));

\section{Appendix 3.2 Extra Summary Data}

Extra Summary Table for Fossil Taxa data

\begin{tabular}{|c|c|c|c|c|c|c|c|c|c|c|c|c|c|c|c|c|}
\hline Identifier & Tax & $\begin{array}{c}\text { Mean } \\
\text { RF }\end{array}$ & SD RF & SE RF & $\begin{array}{c}\text { Max } \\
\text { RF }\end{array}$ & $\begin{array}{c}\text { Min } \\
\text { RF }\end{array}$ & $\begin{array}{c}\text { Mean } \\
\text { PD }\end{array}$ & SD PD & SE PD & Max PD & Min PD & $\begin{array}{c}\text { Mean diff } \\
\text { MPTs }\end{array}$ & $\begin{array}{l}\text { SD diff } \\
\text { MPTs }\end{array}$ & $\begin{array}{l}\text { SE diff } \\
\text { MPTs }\end{array}$ & $\begin{array}{c}\text { Max } \\
\text { diff } \\
\text { MPTs }\end{array}$ & $\begin{array}{c}\text { Min } \\
\text { diff } \\
\text { MPTs }\end{array}$ \\
\hline adephaga & 17 & 5.298 & 5.479 & 1.329 & 23.808 & 2.417 & 21.413 & 18.668 & 4.528 & 89.471 & 10.994 & 49.412 & 180.000 & 43.656 & 736 & -16 \\
\hline adnet & 7 & 6.896 & 6.562 & 2.480 & 15.856 & 0.000 & 18.701 & 16.081 & 6.078 & 40.965 & 0.000 & 36.429 & 58.731 & 22.198 & 138 & 0 \\
\hline ahyong06 & 12 & 4.275 & 3.064 & 0.884 & 9.571 & 1.000 & 12.012 & 7.053 & 2.036 & 23.930 & 3.536 & 11.250 & 31.157 & 8.994 & 109 & -2 \\
\hline apesteg & 6 & 1.667 & 4.082 & 1.667 & 10.000 & 0.000 & 4.387 & 10.746 & 4.387 & 26.321 & 0.000 & -0.500 & 0.548 & 0.224 & 0 & -1 \\
\hline arango7 & 4 & 38.272 & 18.624 & 9.312 & 52.551 & 10.875 & 173.951 & 97.906 & 48.953 & 259.584 & 33.046 & 1697.000 & 1511.345 & 755.673 & 2968 & -16 \\
\hline archostemata & 8 & 6.333 & 4.113 & 1.454 & 11.667 & 2.000 & 13.948 & 7.137 & 2.523 & 23.373 & 6.633 & 1.250 & 1.753 & 0.620 & 5 & 0 \\
\hline asher03 & 39 & 9.312 & 15.193 & 2.433 & 57.387 & 0.000 & 36.614 & 50.947 & 8.158 & 176.385 & 0.000 & 44.923 & 123.377 & 19.756 & 660 & -4 \\
\hline babot & 13 & 10.183 & 5.342 & 1.482 & 17.143 & 2.000 & 23.082 & 9.838 & 2.729 & 37.740 & 6.325 & 4.846 & 6.135 & 1.702 & 20 & 0 \\
\hline beard & 34 & 3.623 & 3.365 & 0.577 & 13.000 & 0.000 & 12.608 & 10.657 & 1.828 & 38.544 & 0.000 & 1.118 & 11.594 & 1.988 & 57 & -7 \\
\hline bisconti & 22 & 3.018 & 1.528 & 0.326 & 6.333 & 1.333 & 11.454 & 4.999 & 1.066 & 23.508 & 5.333 & 1.273 & 4.723 & 1.007 & 18 & -4 \\
\hline blag9 & 7 & 0.714 & 1.496 & 0.565 & 4.000 & 0.000 & 2.176 & 4.661 & 1.762 & 12.490 & 0.000 & 0.286 & 0.756 & 0.286 & 2 & 0 \\
\hline blago4 & 22 & 13.638 & 14.382 & 3.066 & 44.421 & 0.000 & 45.494 & 46.696 & 9.956 & 155.664 & 0.000 & 27.455 & 55.340 & 11.799 & 195 & -4 \\
\hline bloch & 16 & 3.971 & 3.742 & 0.936 & 12.000 & 0.000 & 8.918 & 7.155 & 1.789 & 23.130 & 0.000 & 3.125 & 3.344 & 0.836 & 10 & 0 \\
\hline boess & 17 & 1.739 & 1.197 & 0.290 & 5.250 & 0.000 & 6.595 & 3.486 & 0.846 & 13.477 & 0.000 & -7.824 & 8.017 & 1.945 & 7 & -18 \\
\hline bouetel & 15 & 1.969 & 2.499 & 0.645 & 10.000 & 0.143 & 5.214 & 5.083 & 1.313 & 19.780 & 0.527 & -6.467 & 13.384 & 3.456 & 24 & -24 \\
\hline bourdon & 9 & 0.000 & 0.000 & 0.000 & 0.000 & 0.000 & 0.000 & 0.000 & 0.000 & 0.000 & 0.000 & 0.000 & 0.000 & 0.000 & 0 & 0 \\
\hline brochu & 21 & 7.427 & 0.721 & 0.157 & 8.907 & 6.125 & 18.555 & 1.262 & 0.275 & 20.927 & 16.199 & -61.762 & 191.148 & 41.712 & 432 & -384 \\
\hline claeson & 12 & 15.089 & 11.019 & 3.181 & 28.121 & 2.667 & 53.429 & 35.243 & 10.174 & 95.104 & 12.894 & 1114.500 & 1417.954 & 409.328 & 4176 & 0 \\
\hline clarke9 & 5 & 5.510 & 7.173 & 3.208 & 18.216 & 1.333 & 22.510 & 22.580 & 10.098 & 62.308 & 8.743 & 20.800 & 48.757 & 21.805 & 108 & -2 \\
\hline deng & 26 & 2.123 & 5.808 & 1.139 & 30.000 & 0.000 & 7.903 & 18.704 & 3.668 & 94.446 & 0.000 & -22.077 & 11.913 & 2.336 & 0 & -36 \\
\hline dietz & 6 & 4.768 & 4.267 & 1.742 & 10.857 & 0.000 & 10.456 & 8.185 & 3.341 & 20.672 & 0.000 & 3.500 & 5.394 & 2.202 & 13 & 0 \\
\hline diogo 7 & 7 & 1.683 & 1.440 & 0.544 & 4.625 & 0.313 & 12.416 & 13.793 & 5.213 & 42.500 & 2.050 & 18.286 & 48.379 & 18.286 & 128 & 0 \\
\hline diogo8 & 5 & 2.333 & 0.816 & 0.365 & 3.333 & 1.500 & 11.623 & 2.530 & 1.132 & 14.566 & 8.890 & 0.000 & 0.000 & 0.000 & 0 & 0 \\
\hline evanidae & 16 & 4.841 & 2.835 & 0.709 & 10.667 & 1.000 & 11.329 & 5.465 & 1.366 & 20.740 & 3.082 & 7.438 & 16.621 & 4.155 & 54 & -4 \\
\hline fika & 10 & 10.390 & 6.595 & 2.086 & 22.314 & 3.224 & 27.863 & 12.642 & 3.998 & 47.304 & 13.210 & 549.000 & 923.955 & 292.180 & 1932 & -282 \\
\hline fordyce & 15 & 2.267 & 1.580 & 0.408 & 5.000 & 0.000 & 5.847 & 3.531 & 0.912 & 10.816 & 0.000 & 0.800 & 1.320 & 0.341 & 5 & 0 \\
\hline fried 08 & 5 & 3.133 & 4.134 & 1.849 & 10.000 & 0.000 & 7.375 & 9.232 & 4.129 & 22.450 & 0.000 & 0.400 & 2.074 & 0.927 & 4 & -1 \\
\hline gaffney11 & 27 & 2.309 & 1.383 & 0.266 & 6.333 & 0.000 & 10.663 & 5.942 & 1.143 & 30.512 & 0.000 & 0.407 & 0.971 & 0.187 & 4 & 0 \\
\hline garwood & 3 & 5.675 & 1.217 & 0.703 & 6.896 & 4.462 & 18.448 & 3.250 & 1.877 & 20.515 & 14.702 & -57.667 & 50.063 & 28.904 & 0 & -90 \\
\hline gaudin & 8 & 2.583 & 3.445 & 1.218 & 8.000 & 0.000 & 5.460 & 7.114 & 2.515 & 15.362 & 0.000 & -0.500 & 0.756 & 0.267 & 1 & -1 \\
\hline
\end{tabular}




\begin{tabular}{|c|c|c|c|c|c|c|c|c|c|c|c|c|c|c|c|c|}
\hline gilbert & 16 & 8.002 & 8.630 & 2.157 & 26.000 & 0.000 & 16.062 & 16.582 & 4.145 & 57.649 & 0.000 & -2.813 & 5.332 & 1.333 & 10 & -8 \\
\hline greenwood & 5 & 6.765 & 4.692 & 2.098 & 12.857 & 1.500 & 17.416 & 11.609 & 5.191 & 34.001 & 5.885 & 13.000 & 17.649 & 7.893 & 41 & 0 \\
\hline guanghui & 4 & 4.500 & 3.786 & 1.893 & 10.000 & 2.000 & 8.088 & 4.676 & 2.338 & 14.941 & 5.099 & 0.250 & 0.500 & 0.250 & 1 & 0 \\
\hline hill & 59 & 18.499 & 16.277 & 2.119 & 60.300 & 0.000 & 79.676 & 69.999 & 9.113 & 299.862 & 0.000 & 61.932 & 84.736 & 11.032 & 367 & -4 \\
\hline hilton & 5 & 2.333 & 2.285 & 1.022 & 6.000 & 0.000 & 5.767 & 3.678 & 1.645 & 8.515 & 0.000 & -0.200 & 0.837 & 0.374 & 1 & -1 \\
\hline hospital & 5 & 6.300 & 3.384 & 1.513 & 11.500 & 4.000 & 12.313 & 6.475 & 2.896 & 22.130 & 7.874 & 0.600 & 1.342 & 0.600 & 3 & 0 \\
\hline hurley & 22 & 1.496 & 0.912 & 0.194 & 4.452 & 0.000 & 5.995 & 3.454 & 0.736 & 16.473 & 0.000 & -5.727 & 16.977 & 3.619 & 34 & -24 \\
\hline hutchinson & 11 & 1.431 & 2.660 & 0.802 & 7.385 & 0.000 & 5.455 & 9.627 & 2.903 & 27.293 & 0.000 & -5.364 & 30.303 & 9.137 & 76 & -33 \\
\hline joyce7 & 40 & 7.295 & 7.071 & 1.118 & 38.164 & 1.750 & 32.970 & 23.511 & 3.717 & 135.702 & 10.608 & 57.075 & 132.690 & 20.980 & 540 & -18 \\
\hline kara11 & 22 & 8.716 & 7.155 & 1.526 & 26.100 & 2.000 & 32.442 & 27.660 & 5.897 & 101.945 & 8.246 & 2.909 & 5.318 & 1.134 & 19 & 0 \\
\hline karasawa & 4 & 7.636 & 6.917 & 3.459 & 16.143 & 2.000 & 27.577 & 15.415 & 7.707 & 46.767 & 15.100 & 5.000 & 6.000 & 3.000 & 12 & 0 \\
\hline klug & 11 & 5.742 & 1.430 & 0.431 & 7.617 & 4.000 & 15.165 & 2.311 & 0.697 & 18.937 & 12.374 & 0.727 & 11.909 & 3.591 & 31 & -11 \\
\hline kparrots & 11 & 6.887 & 4.761 & 1.435 & 15.857 & 1.000 & 18.233 & 10.367 & 3.126 & 32.413 & 3.536 & 39.000 & 49.520 & 14.931 & 132 & -4 \\
\hline ksepka & 14 & 4.298 & 2.366 & 0.632 & 8.667 & 0.000 & 14.859 & 7.491 & 2.002 & 28.648 & 0.000 & 0.429 & 0.938 & 0.251 & 3 & 0 \\
\hline lambert_seals & 9 & 3.932 & 2.863 & 0.954 & 9.000 & 0.000 & 8.859 & 5.601 & 1.867 & 14.906 & 0.000 & 4.889 & 4.702 & 1.567 & 14 & 0 \\
\hline lambert13 & 21 & 4.584 & 4.220 & 0.921 & 13.700 & 1.000 & 12.715 & 10.627 & 2.319 & 36.816 & 3.536 & 2.810 & 5.354 & 1.168 & 16 & -3 \\
\hline legg & 98 & 7.380 & 4.850 & 0.490 & 28.667 & 1.333 & 52.794 & 31.540 & 3.186 & 173.264 & 16.147 & 0.388 & 1.842 & 0.186 & 12 & -2 \\
\hline li07 & 10 & 2.600 & 4.018 & 1.271 & 14.000 & 0.667 & 7.922 & 4.956 & 1.567 & 19.131 & 5.055 & 0.600 & 4.006 & 1.267 & 8 & -4 \\
\hline lopezRhizo & 32 & 10.115 & 6.618 & 1.170 & 27.403 & 3.721 & 27.585 & 16.823 & 2.974 & 66.996 & 13.308 & 216.250 & 684.580 & 121.018 & 2838 & -240 \\
\hline luo11 & 59 & 8.360 & 7.101 & 0.924 & 25.500 & 1.000 & 37.783 & 26.655 & 3.470 & 96.703 & 8.246 & 14.492 & 28.352 & 3.691 & 126 & -4 \\
\hline manos & 5 & 6.871 & 4.705 & 2.104 & 14.000 & 2.857 & 16.944 & 8.129 & 3.636 & 28.926 & 9.215 & -10.000 & 5.385 & 2.408 & -3 & -15 \\
\hline mayr05 & 6 & 0.306 & 0.427 & 0.174 & 1.000 & 0.000 & 1.164 & 1.779 & 0.726 & 4.472 & 0.000 & -9.833 & 4.215 & 1.721 & -2 & -13 \\
\hline mayr11 & 5 & 3.100 & 3.286 & 1.470 & 8.500 & 1.000 & 8.841 & 7.688 & 3.438 & 20.330 & 3.317 & 2.200 & 0.447 & 0.200 & 3 & 2 \\
\hline mayrea 10 & 4 & 18.460 & 13.035 & 6.518 & 30.765 & 3.111 & 46.074 & 31.218 & 15.609 & 73.053 & 12.867 & 895.500 & 1227.092 & 613.546 & 2688 & -12 \\
\hline mihalovic & 7 & 1.238 & 1.007 & 0.381 & 2.500 & 0.000 & 4.984 & 4.002 & 1.513 & 9.659 & 0.000 & -0.857 & 2.795 & 1.056 & 4 & -4 \\
\hline oleary & 39 & 10.195 & 10.996 & 1.761 & 37.000 & 0.000 & 45.343 & 49.768 & 7.969 & 168.820 & 0.000 & 0.795 & 1.128 & 0.181 & 4 & 0 \\
\hline perrichot09 & 7 & 1.920 & 0.780 & 0.295 & 2.889 & 0.444 & 4.694 & 1.644 & 0.621 & 6.118 & 1.412 & -3.714 & 9.827 & 3.714 & 12 & -17 \\
\hline pines & 40 & 65.888 & 25.651 & 4.056 & 81.764 & 0.000 & 199.127 & 86.893 & 13.739 & 307.920 & 0.000 & 1458.150 & 1914.778 & 302.753 & 4999 & 0 \\
\hline poyato & 17 & 4.177 & 2.197 & 0.533 & 9.793 & 1.889 & 11.046 & 4.294 & 1.042 & 21.906 & 7.038 & 21.176 & 81.791 & 19.837 & 330 & -15 \\
\hline pradel & 15 & 2.513 & 3.230 & 0.834 & 10.800 & 0.000 & 6.267 & 7.221 & 1.864 & 21.271 & 0.000 & 0.800 & 1.521 & 0.393 & 4 & 0 \\
\hline prideaux & 17 & 2.094 & 2.225 & 0.540 & 7.600 & 0.000 & 11.221 & 11.124 & 2.698 & 39.202 & 0.000 & 0.294 & 0.985 & 0.239 & 4 & 0 \\
\hline puertolas & 39 & 3.273 & 1.778 & 0.285 & 10.500 & 0.833 & 16.097 & 5.776 & 0.925 & 37.041 & 5.949 & 10.487 & 48.726 & 7.802 & 258 & -24 \\
\hline sigwart & 26 & 15.153 & 9.042 & 1.773 & 41.407 & 4.857 & 40.054 & 26.776 & 5.251 & 124.875 & 13.411 & 64.769 & 117.070 & 22.959 & 425 & -12 \\
\hline simm08 & 5 & 1.600 & 1.673 & 0.748 & 4.000 & 0.000 & 5.875 & 5.817 & 2.601 & 13.126 & 0.000 & 1.800 & 1.643 & 0.735 & 3 & 0 \\
\hline skutchas & 11 & 9.101 & 8.432 & 2.542 & 23.500 & 0.000 & 16.210 & 3.299 & 4.010 & 36.889 & 0.000 & 12.091 & 20.447 & 6.165 & 53 & -5 \\
\hline smith 10 & 8 & 4.565 & 8.422 & 2.978 & 25.000 & 0.000 & 20.645 & 29.108 & 10.291 & 85.157 & 0.000 & 0.375 & 6.232 & 2.203 & 15 & -4 \\
\hline smith11 & 6 & 7.347 & 13.893 & 5.672 & 35.241 & 0.000 & 30.602 & 54.774 & 22.362 & 139.595 & 0.000 & 7.000 & 27.408 & 11.189 & 60 & -21 \\
\hline spaul & 35 & 7.968 & 6.010 & 1.016 & 20.776 & 1.250 & 39.135 & 29.098 & 4.918 & 91.198 & 7.814 & 44.286 & 89.303 & 15.095 & 497 & -1 \\
\hline trueb & 12 & 1.435 & 1.791 & 0.517 & 4.889 & 0.000 & 4.085 & 5.158 & 1.489 & 14.397 & 0.000 & 2.083 & 5.282 & 1.525 & 16 & -1 \\
\hline vea & 8 & 3.663 & 3.852 & 1.362 & 13.177 & 2.058 & 15.215 & 9.599 & 3.394 & 38.827 & 10.221 & 680.875 & 588.815 & 208.178 & 1550 & -1 \\
\hline vlihel & 14 & 1.730 & 3.390 & 0.906 & 12.185 & 0.000 & 4.816 & 6.921 & 1.850 & 22.691 & 0.000 & -475.429 & 113.382 & 30.303 & -240 & -596 \\
\hline waterfowl & 10 & 5.224 & 5.986 & 1.893 & 16.085 & 0.000 & 18.021 & 20.195 & 6.386 & 51.989 & 0.000 & 26.800 & 145.031 & 45.863 & 342 & -112 \\
\hline whitlock10 & 4 & 3.558 & 1.064 & 0.532 & 5.143 & 2.889 & 10.972 & 2.735 & 1.367 & 14.832 & 8.674 & 2.750 & 2.217 & 1.109 & 5 & 0 \\
\hline worthy & 11 & 1.916 & 1.987 & 0.599 & 6.000 & 0.000 & 5.999 & 5.545 & 1.672 & 16.108 & 0.000 & 0.273 & 1.849 & 0.557 & 4 & -2 \\
\hline zhang6 & 19 & 2.604 & 3.187 & 0.731 & 9.259 & 0.000 & 8.240 & 9.455 & 2.169 & 26.174 & 0.000 & 6.895 & 10.060 & 2.308 & 31 & -2 \\
\hline
\end{tabular}




\begin{tabular}{|c|c|c|c|c|c|c|c|c|c|c|c|c|c|c|c|c|}
\hline Identifier & Tax & $\begin{array}{c}\text { Mean } \\
\text { RF }\end{array}$ & SD RF & SE RF & $\begin{array}{c}\text { Max } \\
\text { RF }\end{array}$ & $\begin{array}{c}\text { Min } \\
\text { RF }\end{array}$ & $\begin{array}{c}\text { Mean } \\
\text { PD }\end{array}$ & SD PD & SE PD & Max PD & Min PD & $\begin{array}{c}\text { Mean } \\
\text { diff } \\
\text { MPTs }\end{array}$ & $\begin{array}{l}\text { SD diff } \\
\text { MPTs }\end{array}$ & $\begin{array}{l}\text { SE diff } \\
\text { MPTs }\end{array}$ & $\begin{array}{c}\text { Max } \\
\text { diff } \\
\text { MPTs }\end{array}$ & $\begin{array}{c}\text { Min } \\
\text { diff } \\
\text { MPTs }\end{array}$ \\
\hline adephaga & 30 & 7.045 & 5.787 & 1.057 & 24.051 & 2.167 & 25.514 & 14.276 & 2.606 & 60.912 & 10.908 & 153.733 & 421.752 & 77.001 & 1664 & -12 \\
\hline adnet & 16 & 4.875 & 4.641 & 1.160 & 16.000 & 0.000 & 14.127 & 10.616 & 2.654 & 36.056 & 0.000 & 2.188 & 3.834 & 0.958 & 14 & 0 \\
\hline ahyong06 & 15 & 4.542 & 2.906 & 0.750 & 12.444 & 0.000 & 13.387 & 6.395 & 1.651 & 26.712 & 0.000 & 1.133 & 4.051 & 1.046 & 10 & -3 \\
\hline apesteg & 12 & 4.500 & 5.402 & 1.559 & 16.000 & 0.000 & 8.981 & 8.328 & 2.404 & 24.844 & 0.000 & 1.667 & 2.640 & 0.762 & 7 & -1 \\
\hline arango 7 & 64 & 24.975 & 20.035 & 2.504 & 70.400 & 6.875 & 96.312 & 88.748 & 11.093 & 280.286 & 24.409 & 467.125 & 850.837 & 106.355 & 2968 & -16 \\
\hline archostemata & 16 & 6.219 & 3.167 & 0.792 & 13.000 & 2.000 & 15.496 & 6.827 & 1.707 & 33.246 & 6.633 & 0.688 & 1.740 & 0.435 & 7 & 0 \\
\hline asher03 & 28 & 12.266 & 15.075 & 2.849 & 45.564 & 0.000 & 45.862 & 53.586 & 10.127 & 162.240 & 0.000 & 64.607 & 149.796 & 28.309 & 724 & -4 \\
\hline babot & 9 & 8.994 & 4.196 & 1.399 & 18.000 & 4.000 & 21.509 & 9.498 & 3.166 & 41.399 & 11.662 & 4.111 & 3.756 & 1.252 & 11 & 0 \\
\hline beard & 4 & 3.500 & 5.030 & 2.515 & 10.667 & 0.000 & 13.952 & 19.317 & 9.658 & 40.958 & 0.000 & -4.000 & 1.414 & 0.707 & -2 & -5 \\
\hline bisconti & 12 & 2.779 & 1.045 & 0.302 & 5.000 & 1.333 & 10.424 & 3.346 & 0.966 & 18.163 & 4.634 & -0.500 & 3.631 & 1.048 & 8 & -4 \\
\hline blag9 & 10 & 1.117 & 1.863 & 0.589 & 5.000 & 0.000 & 2.791 & 4.411 & 1.395 & 11.263 & 0.000 & -0.200 & 1.033 & 0.327 & 2 & -1 \\
\hline blago4 & 17 & 14.310 & 15.082 & 3.658 & 44.800 & 0.000 & 45.885 & 47.384 & 11.492 & 149.478 & 0.000 & 28.824 & 68.521 & 16.619 & 244 & -7 \\
\hline bloch & 4 & 0.500 & 1.000 & 0.500 & 2.000 & 0.000 & 1.785 & 3.571 & 1.785 & 7.141 & 0.000 & 0.000 & 0.000 & 0.000 & 0 & 0 \\
\hline boess & 5 & 1.460 & 0.464 & 0.207 & 2.000 & 0.857 & 6.366 & 2.716 & 1.215 & 10.569 & 3.527 & -8.400 & 7.503 & 3.356 & 0 & -18 \\
\hline bouetel & 7 & 0.371 & 0.277 & 0.105 & 0.714 & 0.000 & 1.349 & 1.003 & 0.379 & 2.589 & 0.000 & -9.143 & 8.859 & 3.348 & 0 & -20 \\
\hline bourdon & 7 & 0.000 & 0.000 & 0.000 & 0.000 & 0.000 & 0.000 & 0.000 & 0.000 & 0.000 & 0.000 & 0.000 & 0.000 & 0.000 & 0 & 0 \\
\hline brochu10hornedcrocs & 15 & 7.136 & 1.951 & 0.504 & 12.331 & 5.519 & 18.843 & 4.887 & 1.262 & 31.891 & 15.484 & -4.533 & 264.445 & 68.279 & 640 & -324 \\
\hline claeson & 27 & 9.242 & 8.462 & 1.628 & 29.925 & 2.556 & 33.036 & 26.077 & 5.019 & 99.569 & 12.003 & 255.296 & 680.699 & 131.001 & 2304 & -15 \\
\hline clarke9 & 42 & 6.050 & 5.338 & 0.824 & 19.090 & 0.667 & 22.405 & 14.845 & 2.291 & 54.801 & 5.228 & 69.857 & 144.314 & 22.268 & 456 & 0 \\
\hline deng & 5 & 1.257 & 1.745 & 0.780 & 4.000 & 0.000 & 3.483 & 4.675 & 2.091 & 10.770 & 0.000 & -21.600 & 13.428 & 6.005 & -7 & -34 \\
\hline dietz & 10 & 3.652 & 3.049 & 0.964 & 7.667 & 0.000 & 8.195 & 6.560 & 2.074 & 15.095 & 0.000 & 2.900 & 3.213 & 1.016 & 9 & 0 \\
\hline $\operatorname{diogo} 7$ & 73 & 4.242 & 4.357 & 0.510 & 19.725 & 1.125 & 21.768 & 16.817 & 1.968 & 67.758 & 6.914 & 77.370 & 201.753 & 23.613 & 864 & -112 \\
\hline $\operatorname{diog} 08$ & 64 & 3.700 & 2.339 & 0.292 & 12.712 & 0.667 & 18.355 & 10.482 & 1.310 & 52.145 & 3.859 & 9.656 & 22.716 & 2.839 & 120 & -8 \\
\hline evanidae & 5 & 1.800 & 1.804 & 0.807 & 4.667 & 0.000 & 4.495 & 3.665 & 1.639 & 9.774 & 0.000 & 0.000 & 0.000 & 0.000 & 0 & 0 \\
\hline fordyce & 7 & 2.524 & 0.766 & 0.290 & 4.000 & 2.000 & 7.200 & 1.008 & 0.381 & 8.944 & 6.325 & 0.714 & 0.756 & 0.286 & 2 & 0 \\
\hline fried08 & 13 & 3.388 & 2.850 & 0.790 & 7.429 & 0.000 & 7.637 & 5.819 & 1.614 & 15.227 & 0.000 & 3.846 & 7.570 & 2.100 & 26 & -1 \\
\hline gaffney11 & 9 & 2.900 & 1.347 & 0.449 & 5.500 & 1.000 & 13.472 & 5.968 & 1.989 & 23.436 & 4.123 & 0.778 & 1.302 & 0.434 & 3 & -1 \\
\hline garwood & 40 & 8.066 & 2.306 & 0.365 & 15.514 & 4.688 & 24.559 & 3.341 & 0.528 & 34.005 & 18.076 & -9.175 & 47.148 & 7.455 & 192 & -83 \\
\hline gaudin & 8 & 3.000 & 2.765 & 0.977 & 7.000 & 0.000 & 6.315 & 5.828 & 2.061 & 13.982 & 0.000 & 0.625 & 1.188 & 0.420 & 2 & -1 \\
\hline gilbert & 7 & 23.357 & 9.949 & 3.760 & 28.500 & 1.000 & 43.998 & 18.807 & 7.109 & 58.436 & 3.240 & -4.000 & 4.690 & 1.773 & 6 & -8 \\
\hline greenwood & 21 & 9.183 & 5.522 & 1.205 & 17.434 & 1.500 & 21.079 & 12.170 & 2.656 & 46.658 & 5.885 & 8.476 & 15.683 & 3.422 & 49 & -2 \\
\hline guanghui & 11 & 3.091 & 4.425 & 1.334 & 13.333 & 0.000 & 5.561 & 6.754 & 2.036 & 18.646 & 0.000 & 0.455 & 0.820 & 0.247 & 2 & 0 \\
\hline hill & 20 & 7.499 & 11.536 & 2.580 & 35.632 & 0.333 & 32.852 & 44.493 & 9.949 & 135.448 & 2.068 & 25.150 & 52.797 & 11.806 & 209 & 0 \\
\hline hilton & 12 & 3.621 & 3.065 & 0.885 & 9.333 & 0.000 & 9.217 & 7.232 & 2.088 & 21.325 & 0.000 & 2.667 & 4.979 & 1.437 & 17 & 0 \\
\hline hospital & 16 & 7.286 & 4.061 & 1.015 & 13.556 & 2.000 & 16.371 & 8.055 & 2.014 & 29.643 & 6.164 & 2.938 & 3.991 & 0.998 & 12 & 0 \\
\hline hurley & 6 & 1.805 & 0.744 & 0.304 & 3.000 & 0.929 & 6.523 & 2.007 & 0.819 & 9.436 & 4.080 & 10.500 & 13.323 & 5.439 & 28 & -8 \\
\hline hutchinson & 24 & 2.349 & 5.117 & 1.044 & 19.692 & 0.000 & 6.863 & 12.137 & 2.477 & 43.543 & 0.000 & 15.250 & 52.715 & 10.760 & 251 & -20 \\
\hline joyce7 & 22 & 7.312 & 6.217 & 1.326 & 20.333 & 2.000 & 36.245 & 28.383 & 6.051 & 105.489 & 11.356 & 24.000 & 67.012 & 14.287 & 291 & -18 \\
\hline kara11 & 14 & 8.369 & 7.292 & 1.949 & 21.000 & 2.000 & 36.470 & 36.022 & 9.627 & 108.595 & 9.950 & 1.071 & 1.817 & 0.486 & 6 & 0 \\
\hline karasawa & 40 & 2.292 & 2.627 & 0.415 & 14.840 & 0.000 & 12.592 & 11.104 & 1.756 & 52.299 & 0.000 & 1.750 & 7.605 & 1.202 & 48 & -1 \\
\hline klug & 18 & 7.525 & 1.521 & 0.359 & 10.688 & 5.750 & 18.164 & 2.373 & 0.559 & 22.776 & 15.145 & 16.889 & 13.962 & 3.291 & 48 & 0 \\
\hline kparrots & 16 & 3.854 & 4.493 & 1.123 & 14.500 & 0.667 & 10.077 & 8.611 & 2.153 & 30.219 & 2.357 & 2.813 & 5.741 & 1.435 & 18 & -3 \\
\hline
\end{tabular}




\begin{tabular}{|c|c|c|c|c|c|c|c|c|c|c|c|c|c|c|c|c|}
\hline ksepka & 13 & 5.769 & 3.510 & 0.974 & 15.333 & 2.000 & 18.281 & 8.816 & 2.445 & 43.205 & 7.071 & 2.462 & 6.359 & 1.764 & 23 & 0 \\
\hline lambert13 & 6 & 7.079 & 5.667 & 2.313 & 13.714 & 1.500 & 19.018 & 13.836 & 5.648 & 36.879 & 6.036 & 22.500 & 39.348 & 16.064 & 101 & 0 \\
\hline lambert_seals & 6 & 0.167 & 0.408 & 0.167 & 1.000 & 0.000 & 0.553 & 1.354 & 0.553 & 3.317 & 0.000 & -0.833 & 0.753 & 0.307 & 0 & -2 \\
\hline legg & 74 & 8.053 & 4.707 & 0.547 & 27.556 & 3.333 & 49.599 & 27.917 & 3.245 & 226.456 & 27.113 & 0.162 & 0.844 & 0.098 & 6 & 0 \\
\hline li07 & 23 & 2.186 & 4.233 & 0.883 & 21.000 & 0.000 & 7.215 & 7.565 & 1.577 & 34.970 & 0.000 & 2.783 & 6.842 & 1.427 & 24 & -4 \\
\hline lopezRhizo & 6 & 4.551 & 0.629 & 0.257 & 5.758 & 3.902 & 14.389 & 0.959 & 0.392 & 16.234 & 13.415 & 0.000 & 0.000 & 0.000 & 0 & 0 \\
\hline luo11 & 11 & 18.960 & 9.871 & 2.976 & 31.778 & 2.667 & 71.396 & 31.215 & 9.412 & 99.665 & 15.500 & 18.091 & 19.917 & 6.005 & 54 & 0 \\
\hline manos & 21 & 4.045 & 1.443 & 0.315 & 7.333 & 1.556 & 11.040 & 3.578 & 0.781 & 22.880 & 5.967 & 2.714 & 13.020 & 2.841 & 35 & -15 \\
\hline mayr05 & 12 & 1.799 & 1.969 & 0.568 & 5.419 & 0.000 & 4.761 & 4.794 & 1.384 & 13.213 & 0.000 & 6.750 & 26.465 & 7.640 & 72 & -12 \\
\hline mayr11 & 19 & 4.229 & 6.859 & 1.574 & 19.273 & 0.000 & 9.985 & 15.240 & 3.496 & 45.562 & 0.000 & 1.684 & 3.233 & 0.742 & 11 & -1 \\
\hline mayrea10 & 29 & 18.611 & 13.443 & 2.496 & 33.566 & 0.286 & 44.349 & 29.790 & 5.532 & 79.076 & 1.278 & 644.207 & 1225.175 & 227.509 & 4979 & -18 \\
\hline mihalovic & 19 & 6.226 & 5.183 & 1.189 & 13.783 & 0.000 & 16.063 & 12.769 & 2.929 & 39.738 & 0.000 & 12.211 & 17.164 & 3.938 & 46 & -6 \\
\hline perrichot09 & 9 & 2.796 & 0.947 & 0.316 & 4.667 & 1.733 & 6.478 & 1.527 & 0.509 & 8.860 & 4.647 & -14.000 & 6.384 & 2.128 & -2 & -22 \\
\hline pines & 11 & 75.571 & 6.258 & 1.887 & 82.254 & 60.137 & 229.889 & 33.181 & 10.004 & 273.850 & 181.537 & 2001.727 & 2386.223 & 719.473 & 4999 & 4 \\
\hline poyato & 9 & 7.092 & 4.558 & 1.519 & 15.277 & 3.333 & 16.272 & 9.425 & 3.142 & 35.570 & 9.825 & 99.333 & 205.256 & 68.419 & 624 & 0 \\
\hline pradel & 3 & 0.333 & 0.577 & 0.333 & 1.000 & 0.000 & 0.943 & 1.633 & 0.943 & 2.828 & 0.000 & 0.333 & 0.577 & 0.333 & 1 & 0 \\
\hline prideaux & 17 & 2.853 & 2.691 & 0.653 & 8.000 & 0.000 & 14.713 & 12.342 & 2.993 & 37.108 & 0.000 & 0.471 & 1.007 & 0.244 & 3 & 0 \\
\hline puertolas & 11 & 2.886 & 0.539 & 0.163 & 4.343 & 2.333 & 14.426 & 1.645 & 0.496 & 18.609 & 12.575 & 4.545 & 12.136 & 3.659 & 40 & 0 \\
\hline sigwart & 7 & 21.238 & 9.876 & 3.733 & 35.921 & 10.160 & 52.821 & 27.872 & 10.534 & 98.741 & 20.346 & 119.571 & 97.872 & 36.992 & 274 & 4 \\
\hline simm08 & 23 & 1.510 & 1.928 & 0.402 & 5.200 & 0.000 & 5.033 & 6.184 & 1.289 & 16.305 & 0.000 & 1.609 & 2.311 & 0.482 & 7 & 0 \\
\hline skutchas & 10 & 6.313 & 5.460 & 1.727 & 14.647 & 0.000 & 12.567 & 8.702 & 2.752 & 23.604 & 0.000 & 5.700 & 12.230 & 3.867 & 28 & -4 \\
\hline smith10 & 50 & 1.835 & 3.057 & 0.432 & 17.333 & 0.000 & 9.614 & 14.681 & 2.076 & 76.286 & 0.000 & 1.080 & 6.321 & 0.894 & 36 & -3 \\
\hline smith11 & 52 & 9.910 & 10.409 & 1.444 & 32.141 & 0.000 & 44.865 & 46.427 & 6.438 & 132.136 & 0.000 & 54.769 & 115.507 & 16.018 & 514 & -24 \\
\hline spaul & 15 & 8.394 & 7.719 & 1.993 & 27.796 & 1.250 & 33.594 & 28.931 & 7.470 & 96.556 & 8.376 & 207.267 & 699.942 & 180.724 & 2734 & 0 \\
\hline trueb & 8 & 1.146 & 2.274 & 0.804 & 6.500 & 0.000 & 3.574 & 6.723 & 2.377 & 19.276 & 0.000 & 0.750 & 2.188 & 0.773 & 6 & -1 \\
\hline vea & 38 & 3.785 & 1.982 & 0.322 & 8.745 & 1.082 & 15.713 & 6.108 & 0.991 & 32.166 & 5.627 & 187.526 & 648.861 & 105.259 & 2532 & -167 \\
\hline vlihel & 14 & 3.428 & 3.701 & 0.989 & 9.294 & 0.333 & 11.621 & 11.653 & 3.114 & 29.687 & 1.227 & -189.571 & 602.219 & 160.950 & 1182 & -598 \\
\hline waterfowl & 48 & 8.018 & 8.902 & 1.285 & 35.000 & 0.000 & 30.385 & 31.152 & 4.496 & 114.952 & 0.000 & 65.875 & 200.718 & 28.971 & 704 & -121 \\
\hline whitlock10 & 22 & 2.346 & 1.885 & 0.402 & 7.333 & 0.000 & 7.429 & 5.177 & 1.104 & 20.478 & 0.000 & 0.773 & 4.859 & 1.036 & 15 & -4 \\
\hline worthy & 14 & 0.143 & 0.363 & 0.097 & 1.000 & 0.000 & 0.655 & 1.664 & 0.445 & 4.583 & 0.000 & -0.214 & 1.626 & 0.434 & 3 & -2 \\
\hline zhang6 & 11 & 0.766 & 2.230 & 0.672 & 7.429 & 0.000 & 2.386 & 6.113 & 1.843 & 19.902 & 0.000 & 2.000 & 7.746 & 2.335 & 25 & -2 \\
\hline fika & 29 & 15.111 & 11.370 & 2.111 & 50.118 & 2.327 & 36.878 & 27.963 & 5.193 & 155.221 & 10.111 & 958.966 & 928.837 & 172.481 & 1932 & -376 \\
\hline oleary & 46 & 8.401 & 11.029 & 1.626 & 53.333 & 0.000 & 36.985 & 44.349 & 6.539 & 167.573 & 0.000 & 1.130 & 1.881 & 0.277 & 8 & 0 \\
\hline
\end{tabular}




\section{Appendix 5.1 The data matrix used for figure 5.1}

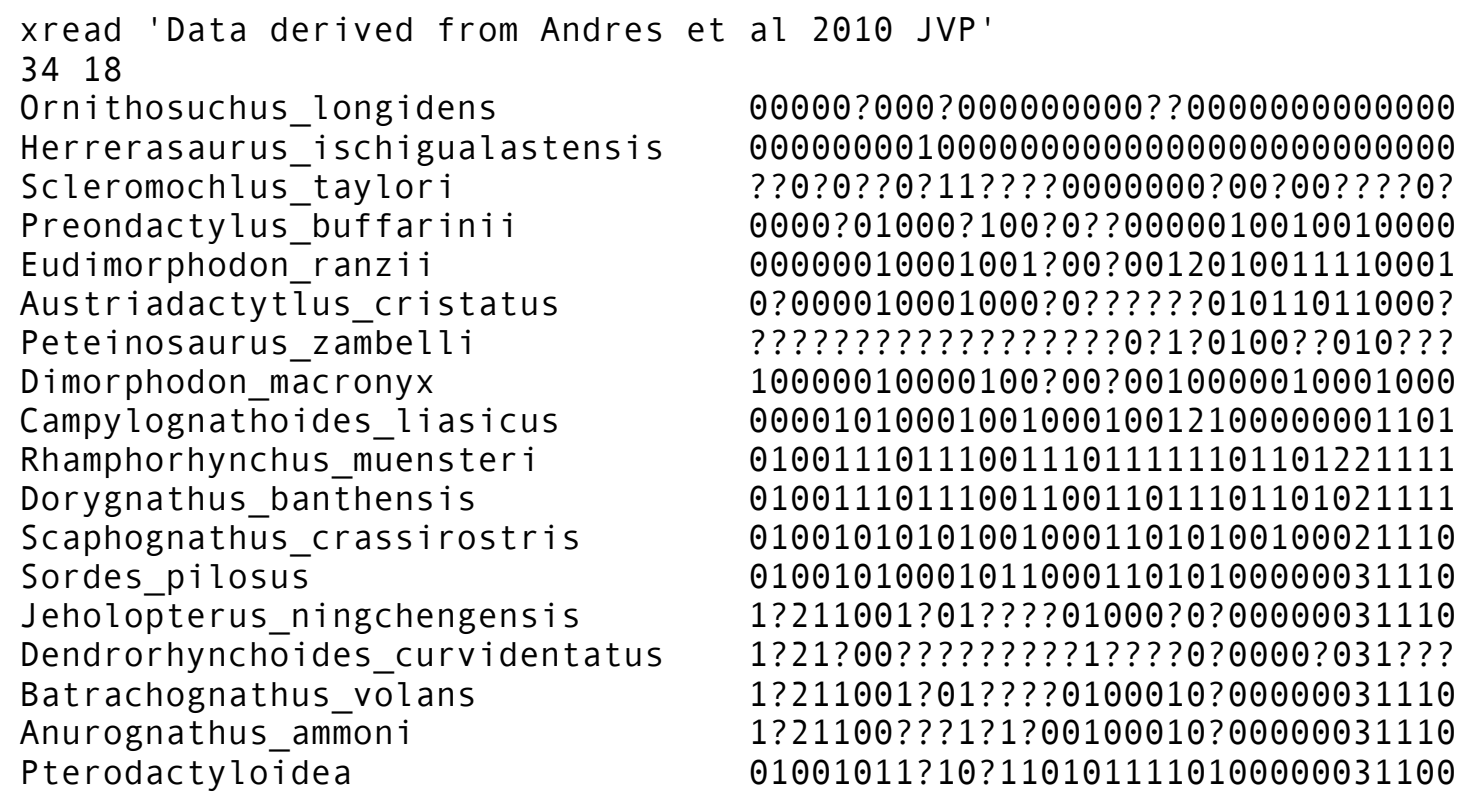
; proc/;

\section{Appendix 5.2 Script to implement selective-permutations ' mher.run '}

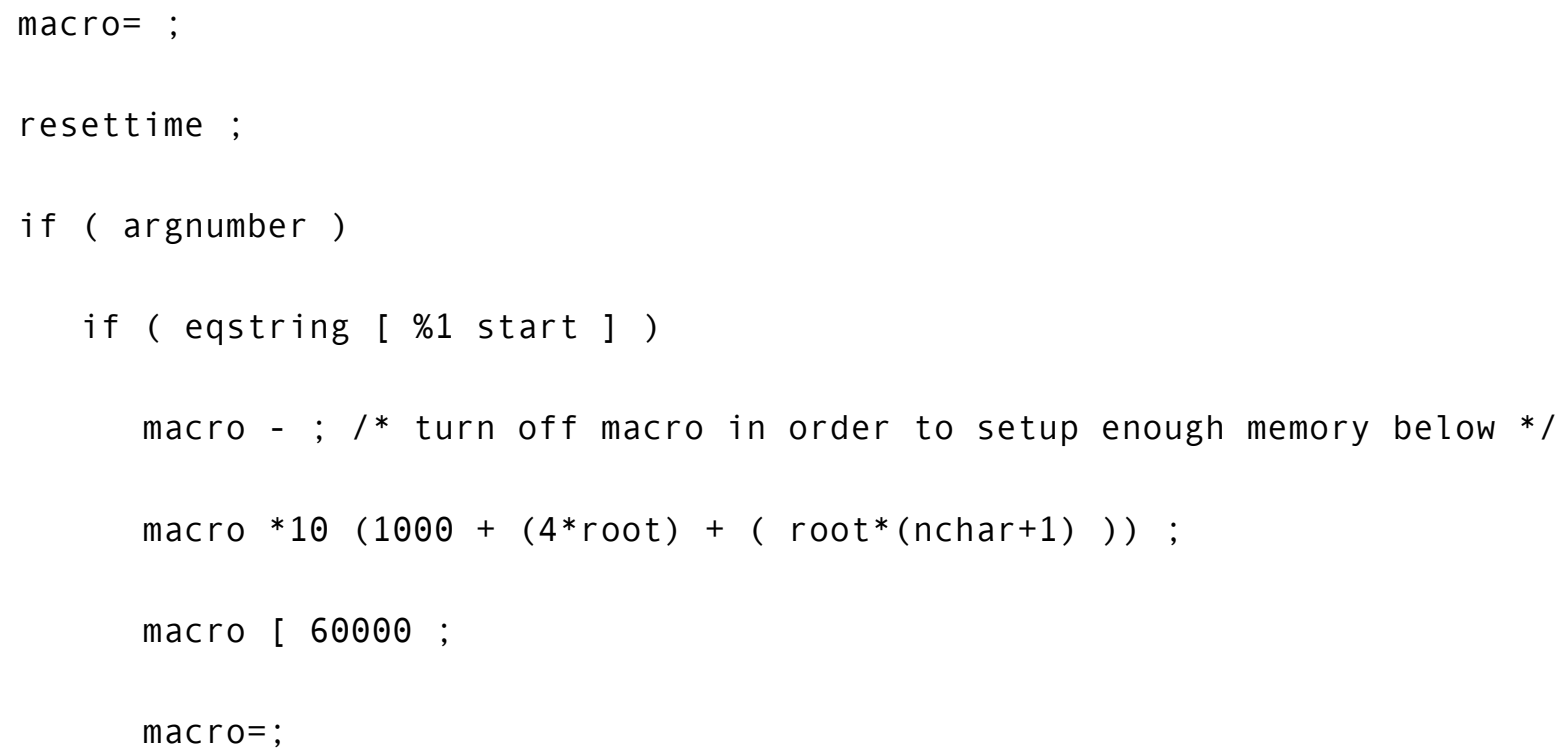




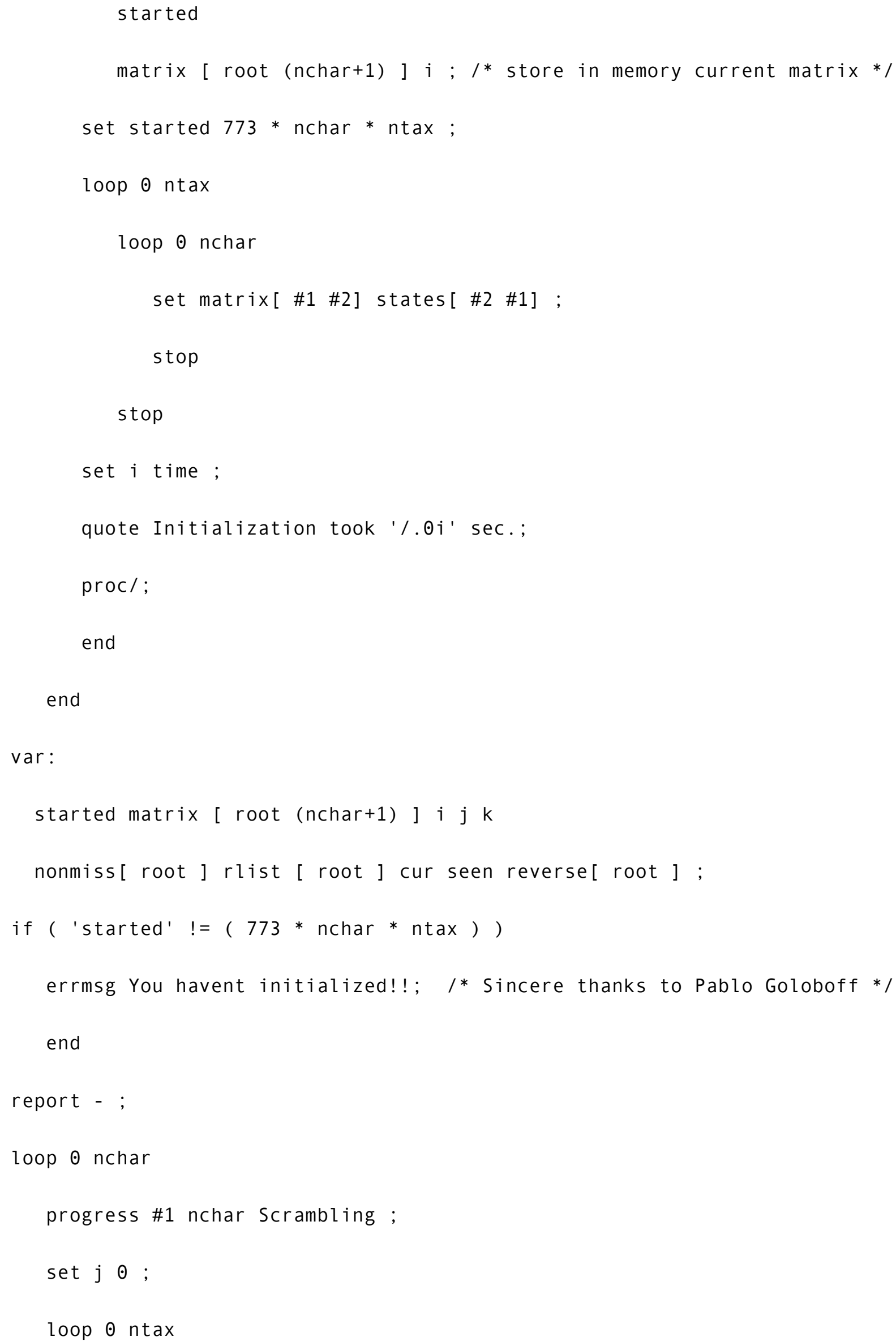


if ( states [\#1 \#2] == missing) continue; end

set nonmiss [ 'j'] \#2 ;

set reverse [ \#2 ] 'j';

set $j++$;

stop

if $\left(!^{\prime} j '\right)$ continue ; end $/ *$ if matrix has no non-missing data $* /$

set rlist randomlist [ 'j'] ;

set $j--$;

loop $\odot{ }^{\prime} j{ }^{\prime}$

set cur 'matrix ['nonmiss ['rlist [ \#2 ]' ]' \#1 ]';

xread $=$ ! \#1

'nonmiss [ \#2 ]' \$bitset 'cur';

stop

stop

progress/;

set $j$ time ;

quote Permuting took '/.0j' sec.;

report $=$;

xread $==$;

proc/ ; 


\section{Appendix 5.3 Script to calculate MHER in one command 'get-mher.sh'}

Pre-requisites:

- a unix operating system (Linux/Mac),

- a reasonably new version of TNT installed that can be called from the terminal by typing 'tnt'. The script relies on it being called tnt, so if it is named anything different it will NOT work. 'mher.run' utilizes some of the newer features in TNT so it will NOT work with older pre-2011 versions of TNT.

Instructions for use:

- place 'get-mher.sh' , 'reps.txt' , and 'mher.run' in the same directory as the .tnt formatted cladistic data matrix you would like to test.

- Make sure your .tnt data file ends with procedure /; Anything else like proc /; or p /; despite being 'valid' TNT shorthand will NOT work with this get-mher.sh bash script.

- 'reps.txt' contains instructions to perform 1000 selective-permutations, which are needed to calculate the modified-MEANNS. I have deliberately hardcoded-in 1000 replications, to prevent people from being tempted to perform a statistically insufficient number of replications e.g. 10 or 100 . However, if you do want to change the number of replications to more or less, this is the file that needs to be changed.

- For ease-of-use and forking, this bundle of files ( 'reps.txt' , 'mher.run' \& 'get-mher.sh' ) used to calculate MHER are provided online on github at: https://github.com/rossmounce/thesis_ESM/tree/master/MHER

The bash script itself including license notice and comments:

\#! /bin/bash

\#This is a bash script to perform the modified Homoplasy Excess Ratio

\#on a dataset of your choice passed to this script as an argument

\#You also need the files 'mher.run' AND 'reps.txt' in the same dir

\#The MIT License (MIT)

\#Copyright (C) 2013 Ross Mounce

\#Permission is hereby granted, free of charge, to any person obtaining a copy of this software and associated documentation files (the "Software"), to deal in the Software without restriction, including without limitation the rights to use, copy, modify, merge, publish, distribute, sublicense, and/or sell copies of the Software, and to permit persons to whom the Software is furnished to do so, subject to the following conditions:

\#The above copyright notice and this permission notice shall be included in all copies or substantial portions of the Software.

\#THE SOFTWARE IS PROVIDED "AS IS", WITHOUT WARRANTY OF ANY KIND, EXPRESS OR IMPLIED, INCLUDING BUT NOT LIMITED TO THE WARRANTIES OF MERCHANTABILITY, FITNESS FOR A PARTICULAR PURPOSE AND NONINFRINGEMENT. IN NO EVENT SHALL THE AUTHORS OR COPYRIGHT HOLDERS BE LIABLE FOR ANY CLAIM, DAMAGES OR OTHER LIABILITY, WHETHER IN AN ACTION OF CONTRACT, TORT OR OTHERWISE, ARISING FROM, OUT OF OR IN 
CONNECTION WITH THE SOFTWARE OR THE USE OR OTHER DEALINGS IN THE SOFTWARE.

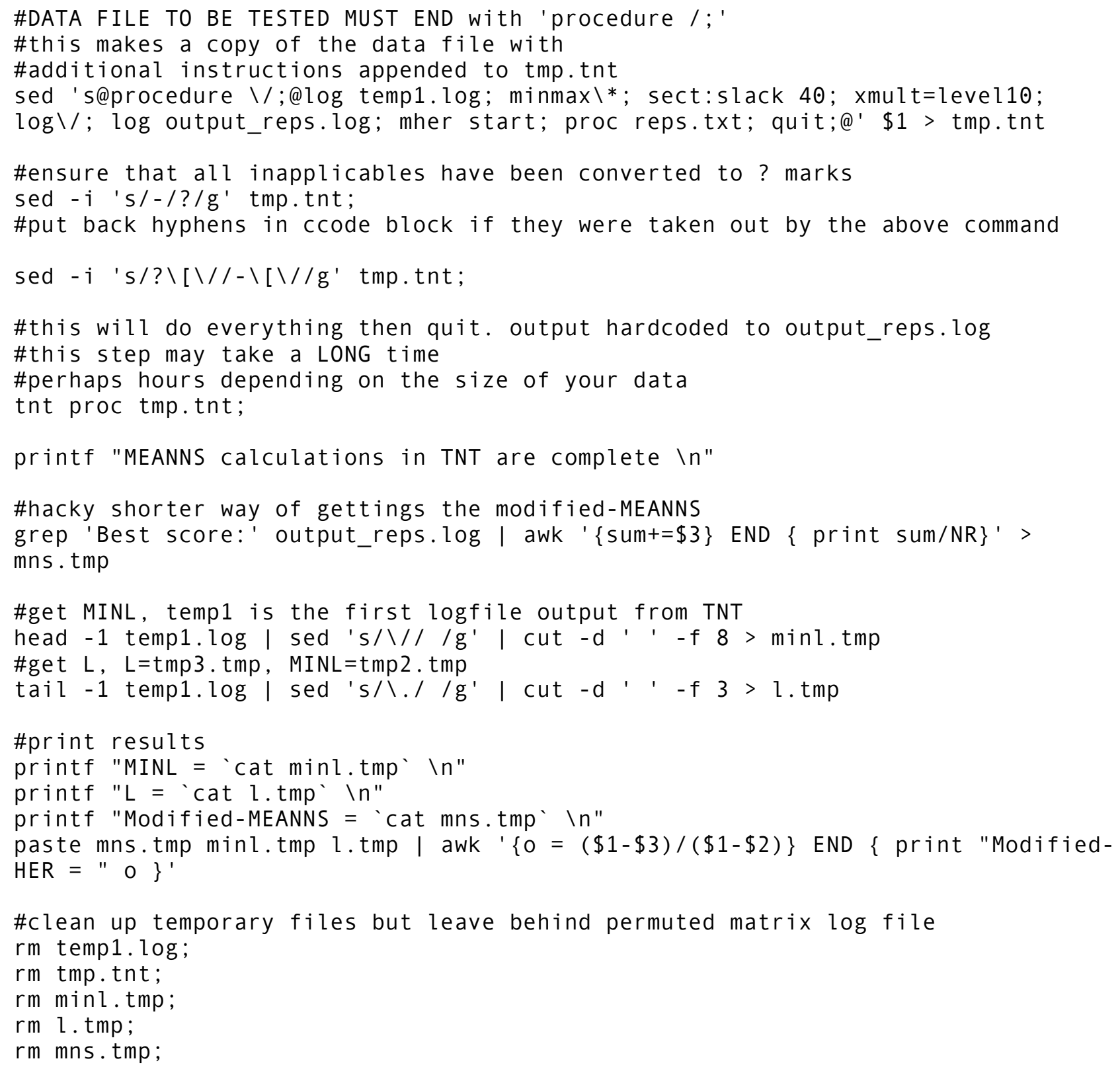

\section{Appendix 6.1 Demonstrating grep}

\section{Sample output from the command}

find. -name "*.txt" -print0 | xargs -0 -n 1000 -P 3 grep -iR -m1 -A 4 -B 4 "paup[^heary]" as applied to the Zootaxa corpus. The four lines of additional context above and below make it very easy to classify the usage of PAUP* with certainty in $99 \%$ of cases as either in the context of a molecular study, or a morphological study. The first two given here are clearly molecular whilst the third is clearly morphology-based. 
./2011/zt02768p031.txt-Phylogenetic analysis. Divergence and polymorphism in cox1 sequences

/2011/zt02768p031.txt-Cox1 sequences from complete mitochondrial genomes of Halisarca harmelini (this study) Halisarca dujardini

./2011/zt02768p031.txt-from White Sea (NC_010212) and Chondrilla aff. nucula (NC_010208) were aligned with partial cox1 sequences

./2011/zt02768p031.txt-of Halisarca dujardini from North Sea (this study) and Chondrilla nucula (Duran \& Rützler 2006) using ClustalW

./2011/zt02768p031.txt:2.0.11 (Larkin et al. 2007). The alignment was trimmed manually to remove terminal gaps associated with incomplete sequences. PAUP* v.4b10 was used to build a neighbor-joining tree based on uncorrected "p" distances.

/2011/zt02768p031.txt-Phylogenetic analysis of demosponge relationships. Amino-acid sequences for Cantharellus cibarius

./2011/zt02768p031.txt-mtDNA were downloaded from

http://megasun.bch.umontreal.ca/People/lang/FMGP/proteins.html; those for Capsaspora owczarzaki mtDNA were provided by Franz Lang (Université de Montréal). Other sequences were derived

./2011/zt02768p031.txt-from the GenBank files: Agelas schmidti EU237475, Amphimedon compressa NC_010201, Amphimedon queenslandica NC_008944, Aplysina fulva NC_010203, Axinella corrugata NC_006894, Chondrilla aff. nucula

./2011/zt02768p031.txt-NC_010208, Callyspongia plicifera NC_010206, Cinachyrella kuekenthali EU237479, Ectyoplasia ferox

./2011/zt02767p040.txt-observed among the O. ishikawae taxa. Gap sites between the $16 \mathrm{~S}$ units of $\mathrm{O}$. ishikawae and the other taxa were

./2011/zt02767p040.txt-treated as missing data in the following analyses. Uncorrected $p$ values (nucleotide changes per compared sequence

$. / 2011 / z t 02767 p 040 . t x t-l e n g t h)$ between taxa were calculated from the resultant alignments. The phylogeny was analyzed by the maximum

./2011/zt02767p040.txt-likelihood (ML) and maximum parsimony (MP) methods. ML and MP analyses were performed using

./2011/zt02767p040.txt:TREEFINDER ver. Oct. 2008 (Jobb 2008) and PAUP 4.10b (Swofford 2003), respectively. For the ML analysis,

./2011/zt02767p040.txt-we applied the J2 (Rodriguez et al. 1990) + gamma (8 categories and shape parameter $=0.32$ ) substitution model,

/2011/zt02767p040.txt-which was estimated using Akaike's information criterion (AIC) implemented in KAKUSAN3 software (Tanabe

./2011/zt02767p040.txt-2007). The robustness of the resultant ML and MP trees were evaluated using bootstrap probabilities calculated

./2011/zt02767p040.txt-from nonparametric bootstrap analyses with 500 pseudoreplications.

./2011/zt02923p047.txt-The character matrix was edited (Table 2) employing the software Nexus Data Editor 
v0.5.0 (Page 2001a). All

./2011/zt02923p047.txt-characters (35 binary and 5 multistate) were set as unordered and equally weighted; the multistate characters were

./2011/zt02923p047.txt-interpreted as "uncertainty", and the gaps were treated as "missing". Trees were rooted by the outgroup method.

./2011/zt02923p047.txt:We carried out a parsimony analysis (Exhaustive Search) in PAUP 4.0b.10 (Swofford 1998-2002), using the

/2011/zt02923p047.txt-default settings of the software. The MaxTrees limit was set to automatically increase from the initial setting. The

./2011/zt02923p047.txt-resulting trees were examined with TreeView 1.6.6 (Page 2001b) and TreeGraph2 (Stöver \& Müller 2010).

$. / 2011 / z t 02923 p 047 . t x t-$

./2011/zt02923p047.txt-30 · Zootaxa 29232011 Magnolia Press

\section{Appendix 6.2 Demonstrating search queries}

Search terms and corresponding URLs used to generate the results presented in Table 4:

MAS: PLOS ONE, 2006-2009, phylip

http://academic.research.microsoft.com/Detail?searchtype=4\&query=year\%3E \%3d2006\%20year\%3C\%3d2009\%20jour\%3a\%28plos\%20one\%29\%20phylip

MS: Zootaxa, (all years), phylogeny

[hits published later than 2013-07-11, outside the corpus scope were manually removed from the count after the search results were returned]

http://www.mendeley.com/research-papers/search/?query=phylogeny+AND+published_in \%3AZootaxa

WoK: Topic= $\left(\right.$ phylogen $\left.{ }^{*}\right)$ AND Publication Name=(plos one) Timespan=2006-2009.

Search language $=$ Auto

http://apps.webofknowledge.com/summary.do?

SID=T2FmvrZ1heYnZ9rNrIG\&product=UA\&qid=1\&search_mode=GeneralSearch

Scopus: query: ALL(phylogeny) AND SRCTITLE(plos one) AND PUBYEAR > 2005 AND PUBYEAR $<2010$

http://www.scopus.com/results/results.url?sort=plf-

f\&src $=s \& s t 1=$ phylogeny\&searchTerms $=P L O S+O N E \% 3 f \% 21 \% 22$ *

$\% 24 \&$ sid $=7053$ A17CC766D7A14AC3E36512AC1E40.ZmAySxCHIBxxTXbnsoe5w

\%3a80\&sot=b\&sdt=b\&sl=75\&s=ALL\%28phylogeny\%29+AND+SRCTITLE\%28PLOS+ONE $\% 29+A N D+P U B Y E A R+\% 3 E+2005+A N D+P U B Y E A R+$

\%3C+2010\&origin=searchbasic\&txGid=7053A17CC766D7A14AC3E36512AC1E40.ZmAy SxCHIBxxTXbnsoe5w\%3a8 
GS: with all of the words: phylogeny , Return articles published in: PLOS ONE, Return articles dated between: 2006 - 2009

http://scholar.google.co.uk/scholar? as $\mathrm{q}=$ phylogeny\&as $\mathrm{epq}=$ \&as $\mathrm{oq}=$ \&as $\mathrm{eq}=$ \&as occt=any\&as sauthors=\&as publication =PLOS+ONE\&as ylo=2006\&as yhi=2009\&btnG=\&hl=en\&as sdt=0\%2C5

\section{Appendix 6.3 Google Scholar finds IR copies}

Screenshot of the search results returned by GS for the 'nona' search which anecdotally provide evidence that GS generally only finds terms in the full-text of the paper if the fulltext of the paper has been optionally and additionally deposited by the authors on 'academic web addresses' that are crawled by Google. In this example we can see many examples of this e.g. Huber's 2007 Zootaxa paper which is freely available from the University of Bonn repository here: http://www.unibonn.de/ bhuber1/PDFs/Huber_2007_Anansus_Nyikoa.pdf

[PDF] Description of Ossinissa, a new pholcid genus from the Canary Islands (Araneae: Pholcidae) D Dimitrov, C Ribera - Zootaxa, 2005 - gwu.edu Paperpile 1

... The numerical cladistic analysis was done using NONA, version 2 (Goloboff 1999) and

Pee-Wee, version 2.8 (Goloboff 1997).... Running NONA with hold 10000 and mult*1000

gave as a result 81 most parsimoni- ous trees with 94 steps. ...

Cited by 2 Related articles All 5 versions Cite More

[PDF] DNA barcodes: Evaluating the potential of COI to diffentiate closely related species of Elachista [PDF] from psu.edu

(Lepidoptera: Gelechioidea: Elachistidae) from Australia

L Kaila, G Stahls - Zootaxa, 2006 - Citeseer Paperpile

... Phylogenetic relationships of included terminals were estimated (using equal weights) using

the parsimony program NoNa vs. ... Bremer (Bremer 1988, 1994) values were estimated using

NoNa and Jackknife support values using WinClada (Nixon 2002). ...

Cited by 31 Related articles All 4 versions Cite More-

[PDF] Two new genera of small, six-eyed pholcid spiders from West Africa, and first record of Spermophorides for mainland Africa (Araneae: Pholcidae)

[PDF] from uni-bonn.de

BA Huber - Zootaxa, 2007 - uni-bonn.de Paperpile

... The numerical cladistic analysis was done using NONA, version 2.0 (Goloboff 1993). ... See

Relationships for details of the analysis. Relationships Using NONA with hold/50, mult* 100 , and

amb- results in six most parsimonious cladograms with a length of $160(\mathrm{Cl}=40 ; \mathrm{RI}=78) . \ldots$

Cited by 9 Related articles All 3 versions Cite More -

[PDF] A cladistic analyses of the Neotropical genus Sepedonea Steyskal (Diptera: Sciomyzidae)

L Marinoni, WN Mathis - Zootaxa, 2006 - Citeseer Paperpile

... The monophyly of the genus is confirmed, as is the genus' sister-group relationship to

Sepedomerus Steyskal, 1973. The cladistic analysis was done using NONA and a matrix of 27

adult morphological characters, including structures of the male and female terminalia. ...

Cited by 4 Related articles All 7 versions Cite More-

[PDF] A new anchialine shrimp of the genus Procaris from Christmas Island: the first occurrence of the [PDF] from nhm.org

Procarididae in the Indian Ocean (Crustacea: Decapoda: .

AJ Bruce, PJF Davie - Zootaxa, 2006 - decapoda.nhm.org Paperpile

... For the present analyses we used NONA 2.0 (Goloboff 1997) through Winclada 1.00 .08 (Nixon

2002) using settings mult* $n=25$, with 1000 replications, and 10 starting trees per replication, all

characters were treated as unordered. ... Goloboff, P. (1997) NONA Version 2.0. ...

Cited by 6 Related articles All 3 versions Cite More-

[PDF] Description of the females of Oxysoma itambezinho Ramirez and Monapia tandil Ramirez, and [PDF] from macn.gov.ar their effects on the generic relationships of Gayennini (Araneae....

MJ Ramirez, MJ Ansaldi, AF Puglisi - Zootaxa, 2004 - aracnologia.macn.gov.ar

... The cladistic analysis was conducted with the same parameters and search strategies as in

Ramirez (2003), using implied weights with Pee-Wee, and equal weights with NONA (Goloboff

1993b, 1997). ... Cladistics, 9, 83-91. Goloboff, PA (1993b) Nona version 2.0. ... 


\section{Appendix 7.1 Phylogenetic position of Diania challenged}

This section was co-authored with Matthew A. Wills and published (Mounce \& Wills, 2011)

Formatting has been changed to avoid copyright infringement with the published version

Liu et al. (2011a) describe a new and remarkable fossil, Diania cactiformis. This animal apparently combined the soft trunk of lobopodians (a group including the extant velvet worms in addition to many Palaeozoic genera) with the jointed limbs that typify arthropods.

They go on to promote Diania as the immediate sister group to the arthropods, and conjecture that sclerotized and jointed limbs may therefore have evolved before articulated trunk tergites in the immediate arthropod stem. The data published by Liu et al. (2011a) do not unambiguously support these conclusions; rather, we believe that Diania probably belongs within an unresolved clade or paraphyletic grade of lobopodians. Without taking issue with the interpretation of Diania offered by Liu et al. (2011a), or of the manner in which they coded their characters, we were nonetheless unable to derive their cladogram

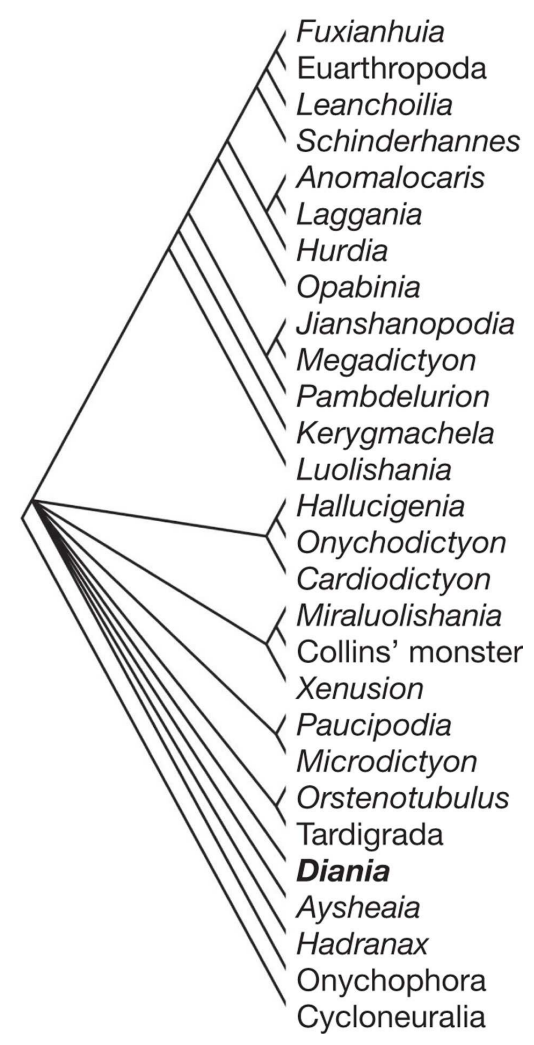
optimally from the data published. Moreover, we could not replicate their results using any other plausible optimality criteria, or by varying additional parameters not specified by the authors. Liu et al. (2011a) report analysing their data in PAUP* $^{*}$ under maximum parsimony and with implied weights using $\mathrm{k}=2$ (a rather arbitrary choice), but do not mention any other assumptions (for example, the imposition of character order). They obtained three most parsimonious trees, each of 130 steps. Straightforward replication of their stated settings yields 13 trees of just 90 steps each, the strict consensus of which is illustrated (Fig. 7.1).

Figure 7.1 The strict consensus of 13 most parsimonious trees $(\mathrm{L}=90)$ obtained from the published data and settings specified by the authors.

Why such a difference? Several of their characters contained inapplicable or gap codings. 
These appear where a 'daughter' character is logically contingent upon the state of a 'parent', and cannot be coded when the parent is absent. For example, character 6 (position of frontal appendage) can only be coded in taxa that possess a frontal appendage (character 5 ) in the first instance (such that a " 0 " for character 5 necessitates a "-" for character 6). In morphological analyses such as this, inapplicable states are usually assumed to have no bearing on the analysis, being reconstructed passively in the light of known states. In analyses of nucleotide data, by contrast, gaps may alternatively be construed as a fifth and novel state, because shared deletions from some ancestral sequence may actually be informative. If this assumption is made with morphological data, however, all the logically uncodable states in a character are initially assumed to be homologous, and a legitimate basis for recognizing clades. At best, this assigns double weight a priori to absences in the 'parent' character (because the daughter is always contingent), and at worst is positively misleading. This is the approach that we believe Liu et al. (2011a) may have taken. Reanalysis of their data using 'gapmode = newstate' combined with 'collapse $5=$ MinBrLen' settings in PAUP* produced some optimal trees of 130 steps. However, we were still unable to replicate the relationships shown in their Fig. 4 , even when varying $\mathrm{k}$ between 0 and 10 . Rather we either resolved Diania in a basal polytomy, or slightly higher in the tree but separated from the arthropods by at least five nodes. At best, therefore, the position of Diania is highly labile and extremely sensitive to the precise methods used. We certainly feel that it is premature to draw conclusions regarding its supposedly pivotal position in the evolution of arthropods. However, our reanalyses do not challenge the more general conclusions of Liu et al. (2011a): namely that the full complement of arthropod characters were probably acquired piecemeal and possibly convergently. Many closely allied groups exploited successfully some but not all of the characters that typify the arthropod crown group. Only in retrospect do we discern a single, ladder-like trajectory through what was really a much more eccentrically branching bush. 